\title{
USING TOMOGRAPHY AND COMPUTATIONAL FLUID DYNAMICS TO STUDY THE PERFORMACE OF A MAXBLEND IMPELLER FOR SOLID-LIQUID MIXING OPERATIONS
}

By

\author{
Prakash Mishra
}

Bachelor of Engineering, Visveswaraiah Technological University, Belgaum, Karnataka, India, 2008

\author{
A Thesis \\ Presented to Ryerson University \\ in Partial Fulfilment of the Requirements for the Degree of \\ Master of Applied Science \\ in the Program of Chemical Engineering
}

Toronto, Ontario, Canada, 2016

(C) Prakash Mishra, 2016 


\section{Author's Declaration}

I hereby declare that I am the sole author of this thesis. This is a true copy of the thesis, including any required final revisions, as accepted by my examiners.

I authorize Ryerson University to lend this thesis to other institutions or individuals for the purpose of scholarly research.

I further authorize Ryerson University to reproduce this thesis by photocopying or by other means, in total or in part, at the request of other institutions or individuals for the purpose of scholarly research.

I understand that my thesis may be made electronically available to the public.

Prakash Mishra 


\begin{abstract}
Prakash Mishra

Using tomography and computational fluid dynamics to study the performance of a Maxblend impeller for solid-liquid mixing operations

MASc, Chemical Engineering, Ryerson University, Toronto, 2016
\end{abstract}

A thorough literature review suggests that no comprehensive research work has been done regarding the characterization of the local solid concentrations in a slurry reactor equipped with a Maxblend impeller. The aim of this research work was to assess the mixing performance of a Maxblend impeller in a slurry reactor through electrical resistance tomography (ERT) and computational fluid dynamics (CFD). The mixing efficiency of the Maxblend impeller for solidliquid mixing operation was compared to those measured for the A200 (an axial-flow impeller) and the Rushton turbine (a radial-flow impeller). The tomography images were employed to assess the particles distribution inside the slurry reactor. The CFD model was created using the Eulerian and Eulerian (E-E) method, standard $k-\varepsilon$ turbulence model, and sliding mesh (SM) technique for simulating the two-phase fluid flow, turbulence effects, and stirrer rotation, respectively. The validated CFD model was utilized to obtain the particle concentration profiles and to determine the local particle distributions attained by the Maxblend impeller. The data were utilized to analyze the impacts of various important parameters such as the agitation speed, particle concentration, particle diameter, specific gravity of the particle, and the use of baffles on the mixing efficiency of the Maxblend impeller in terms of the extent of homogeneity and mixing index. The particle distribution in the slurry reactor furnished with a Maxblend impeller was also assessed through clouding height and just suspended agitation speed approaches in this study. The results from this study showed that the assessment of the optimum impeller speed is extremely important to enhance the local mixing quality in the mixing vessel. Experimental tests demonstrated that maximum homogeneity attained by the Maxblend impeller was higher than those for the A200 and Rushton impellers. 


\section{Acknowledgements}

First of all, I would like to extend my deep gratitude and appreciation to my supervisor Dr. Farhad Ein-Mozaffari for his invaluable advice, guidance and support throughout my research work. I could not have imagined having a better mentor and advisor for my MASc study. I acknowledge the help of administrative staffs, and engineering staffs in the Department of Chemical Engineering at Ryerson University. I would like to extend my sincere thanks to all my colleagues in the Department of Chemical Engineering at Ryerson University for their kind help and encouragement. I acknowledge Ontario Graduate Scholarship (OGS), Queen Elizabeth II Graduate Scholarships in Science and Technology (QEII -GSST), and Natural Sciences and Engineering Research Council of Canada (NSERC) for the financial support during my research work. Sincere thanks to my parents for their unconditional love and support. Finally, special thanks to my wife and daughter for their cooperation during my study. 


\section{Dedicated to}

My mother Late Ratneshwari Mishra

for her blessings 


\section{Table of Contents}

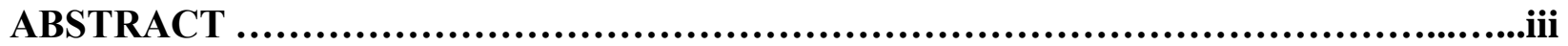

ACKNOWLEDGEMENTS ............................................................................................................iv

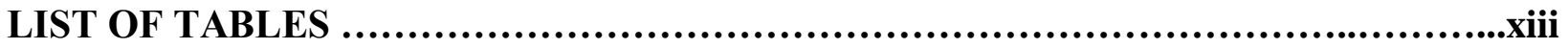

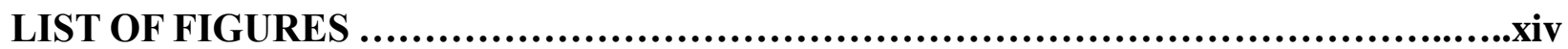

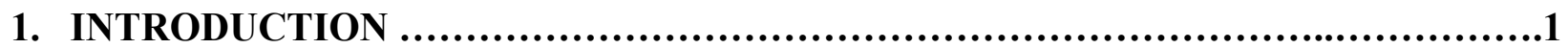

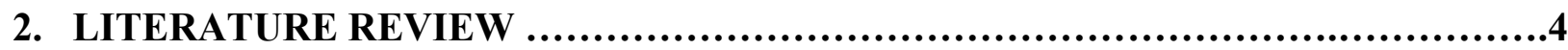

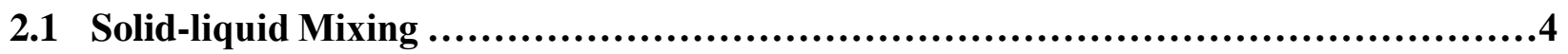

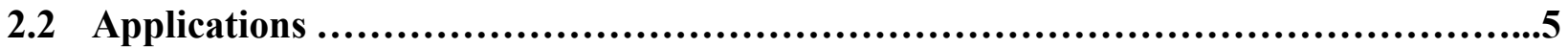

2.3 Geometric Design and Considerations in Mixing ....................................

2.4 Characterization of Solid Distribution ...............................................

2.4.1 Models and Methods for Characterizing Just Suspension Speed .....................10

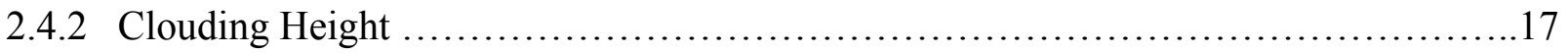

2.4.3 One Dimensional Sedimentation and Dispersion Model ..............................18

2.4.4 Dispersion and Distribution of Floating Solids ...................................23

2.5 Factors Influencing Solid-liquid Suspension ......................................24

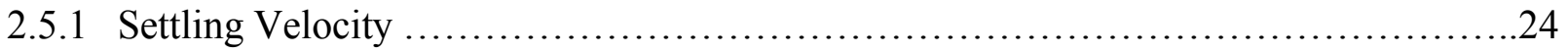

2.5.2 Hindered Settling Velocity ................................................... 25 
2.5.3 Particle Size Distribution

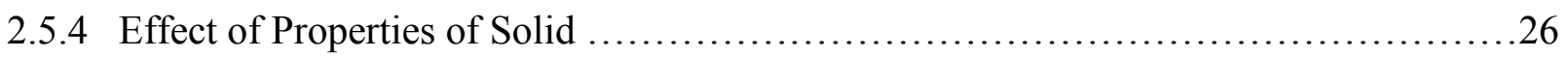

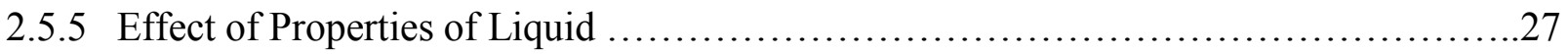

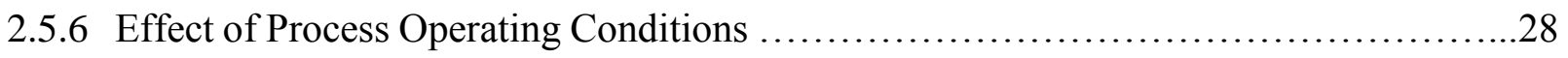

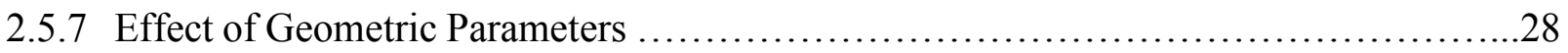

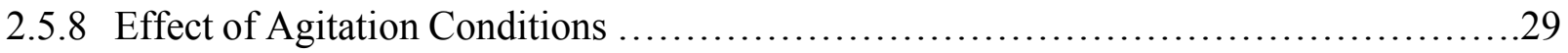

2.6 Solid Concentration Measurement Techniques .......................................32

2.6.1 Invasive Measurement Techniques .............................................. 32

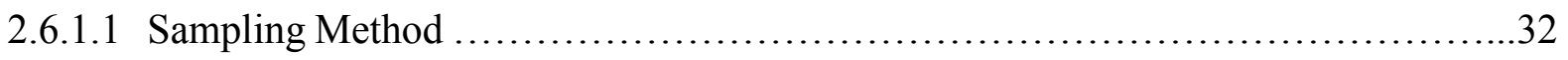

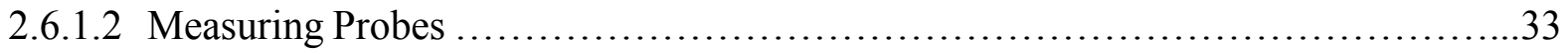

2.6.1.2.1 Impedance Techniques .................................................33

2.6.1.2.2 Optical Techniques ....................................................... 34

2.6.1.2.3 Acoustic Techniques ..................................................... 34

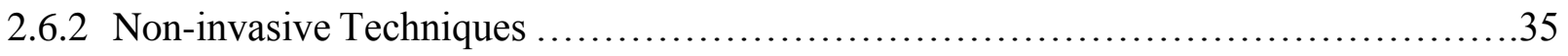

2.6.2.1 Light Techniques .............................................................

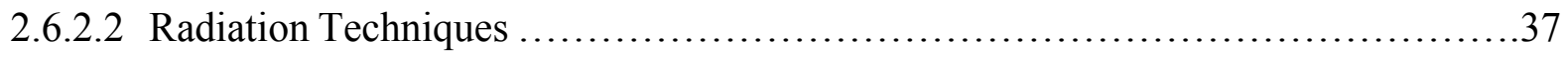

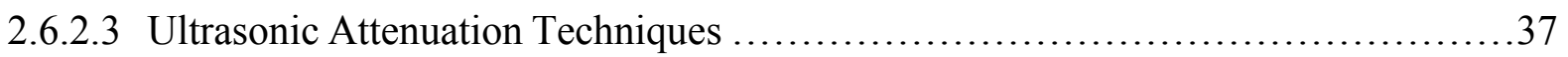

2.6.2.4 Radioactive Particles Tracking Methods ...................................... 38 


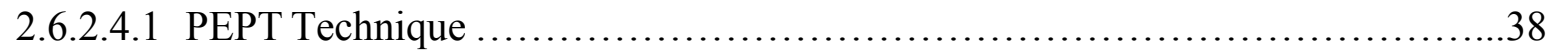

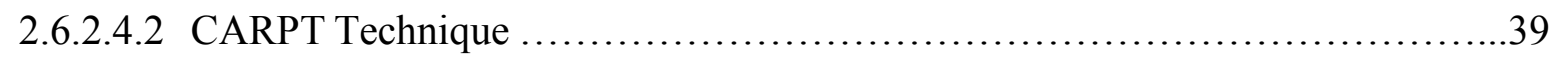

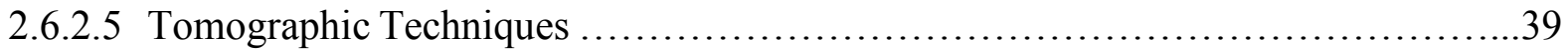

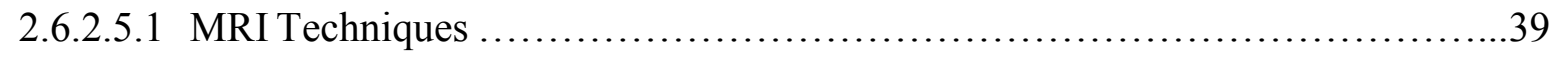

2.6.2.5.2 Computed Tomography ..................................................40

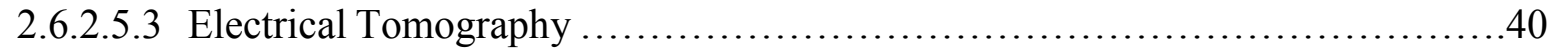

2.7 Forces Acting on Solid Particles ...................................................44

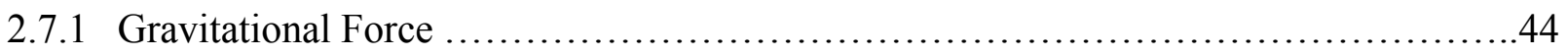

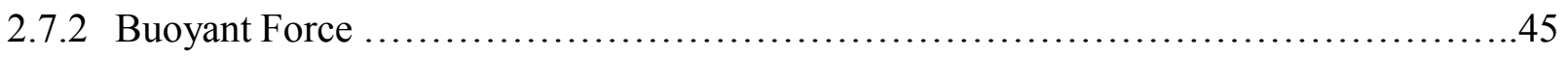

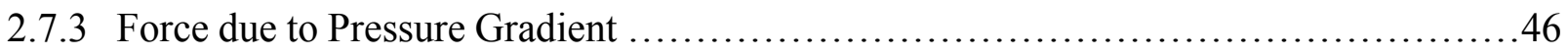

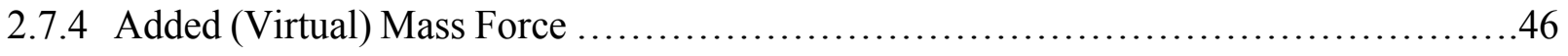

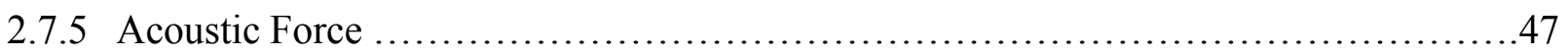

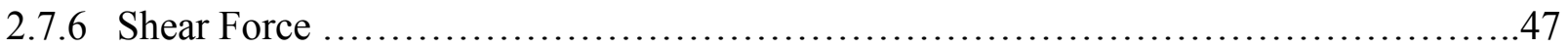

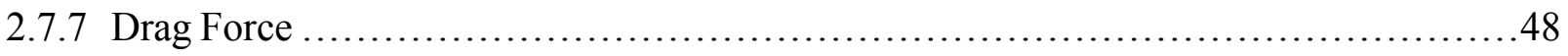

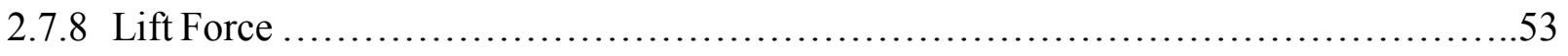

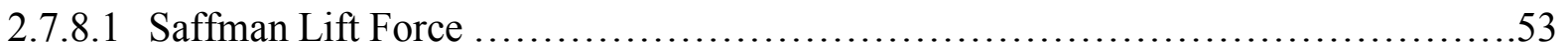

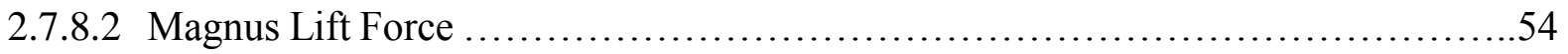

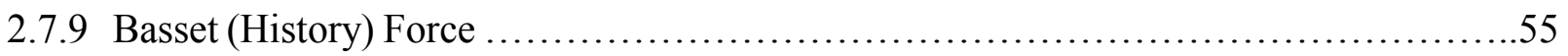


2.8.1 Eulerian and Lagrangian Approach for Single Phase Flow .56

2.8.2 Selection of Suitable Approach for Modelling Solid-liquid Suspension Flow .58

2.8.3 Eulerian-Eulerian Approach 60

2.8.4 Solid-liquid Exchange Coefficient .62

2.8.5 Eulerian-Lagrangian Approach .64

2.8.6 Fast or Equilibrium Eulerian Approach .66

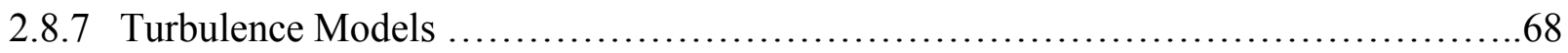

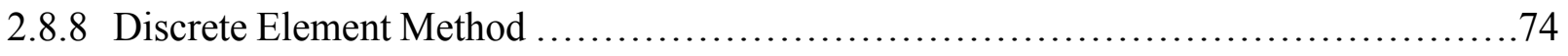

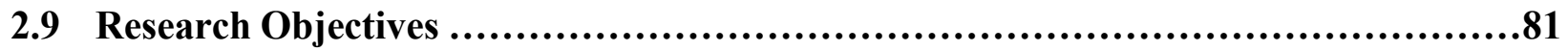

3. EXPERIMENTAL SETUP AND METHODOLOGY .................................82

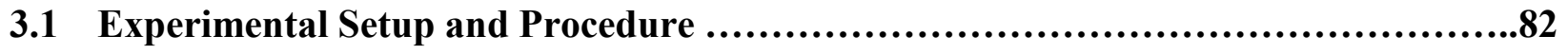

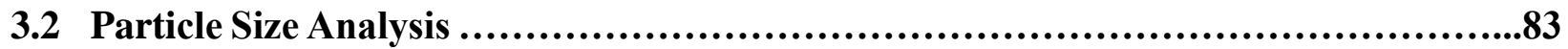

3.3 Electrical Resistance Tomography (ERT) ….....................................85

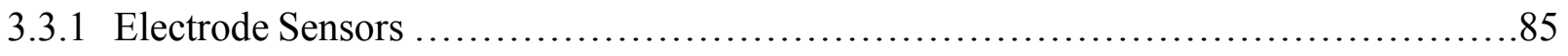

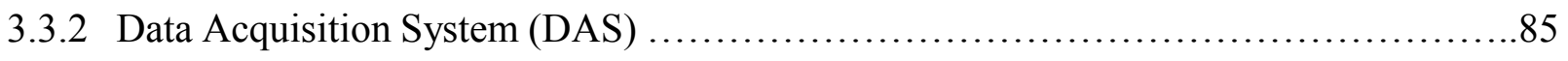

3.3.3 Image Reconstruction System (Host Computer) ...................................86

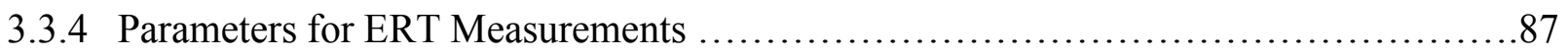


3.5 Tests Conditions ....................................................................88

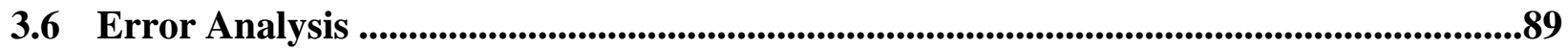

4. CFD SIMULATION AND NUMERICAL MODELING .............................90

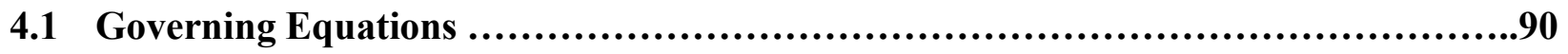

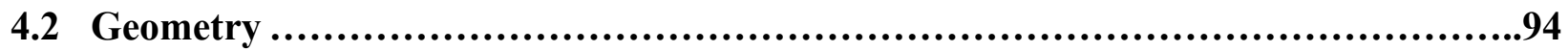

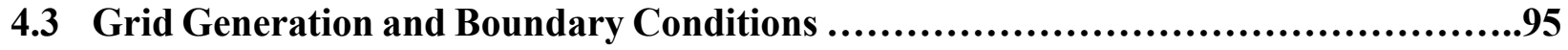

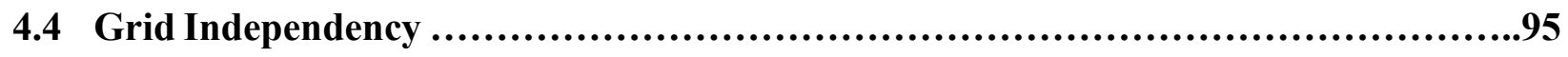

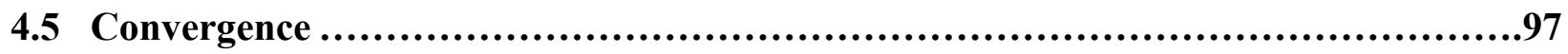

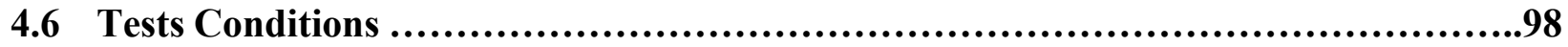

5. RESULTS AND DISCUSSIONS ...............................................99

5.1 Using Tomograms to Assess the Local Solid Concentrations in a Slurry Reactor Equipped with a Maxblend Impeller .....................................................99

5.1.1 Axial Particle Concentration Profiles ...................................... 103

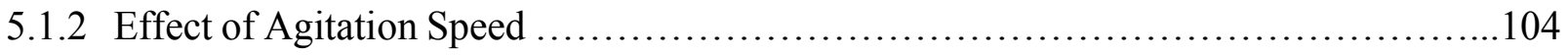

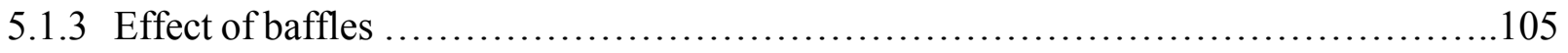

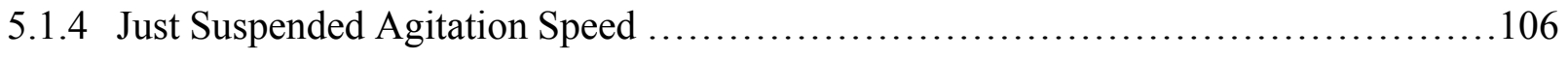

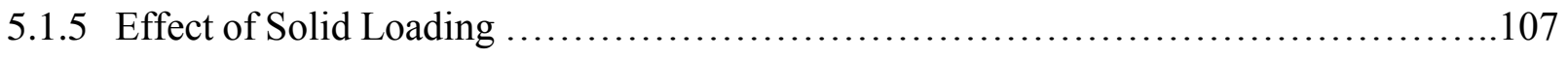

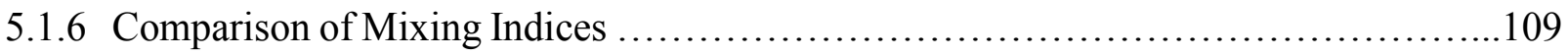


5.1.7 Effect of Average Particle Size

5.1.8 Influence of Stirrer Type

5.1.9 Effect of Stirrer Clearance

.115

\subsection{Using Computational Fluid Dynamics to Analyze the Performance of the Maxblend Impeller in Solid-liquid Mixing Operations ..............................................117}

5.2.1 Qualitative Validation ........................................................ 117

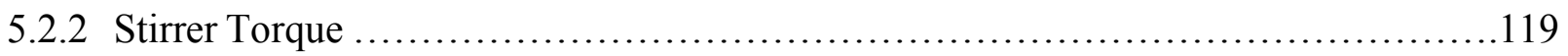

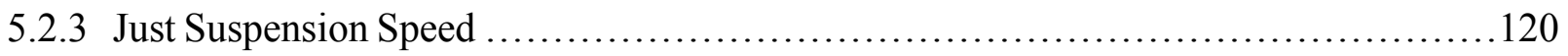

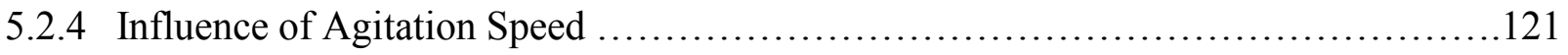

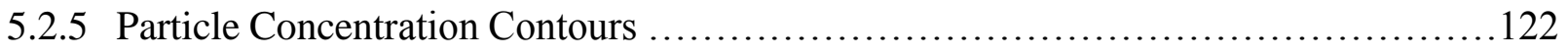

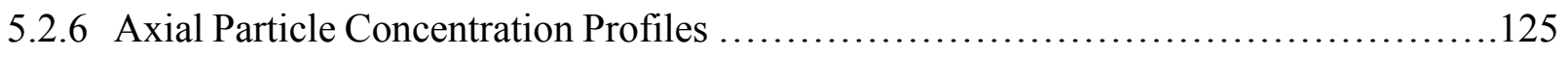

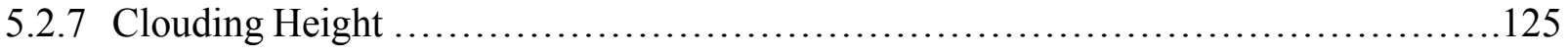

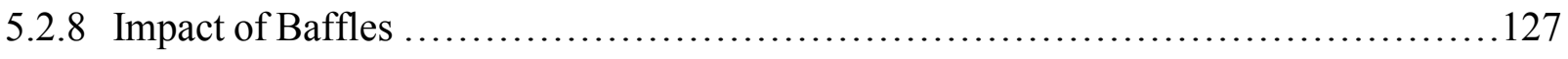

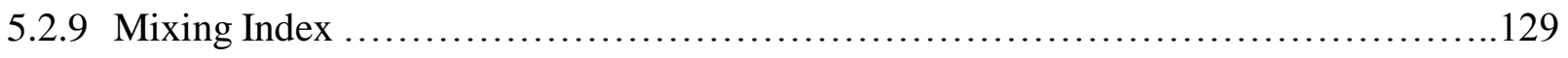

5.2.10 Effect of Particle Diameter ................................................... 131

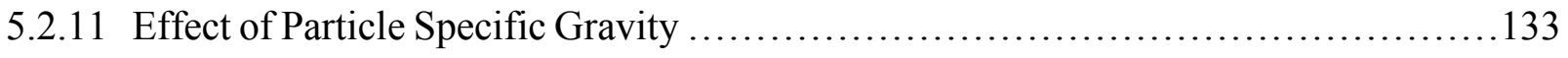

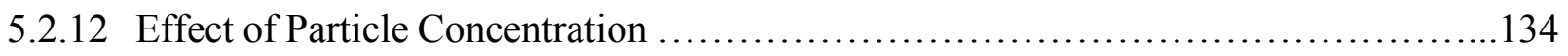

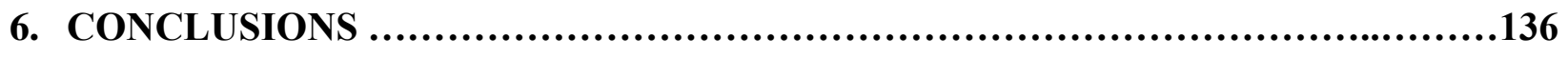

6.1 Recommendations for Future Research Work ....................................138 
NOMENCLATURE

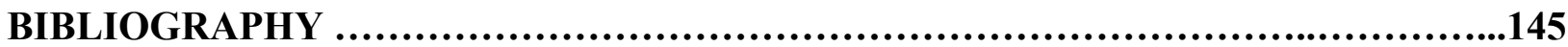




\section{List of Tables}

Table (2.4-1). A brief summary of suspension models .................................. 16

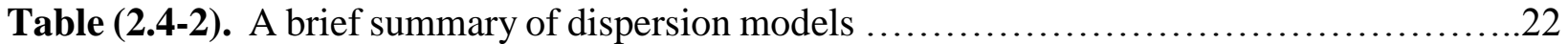

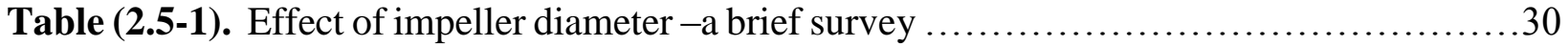

Table (2.6-1). A brief literature survey of ERT in solid-liquid mixing $\ldots \ldots \ldots \ldots \ldots \ldots \ldots \ldots . \ldots 42$

Table (2.7-1). Drag coefficient correlations for spherical particles $\ldots \ldots \ldots \ldots \ldots \ldots \ldots \ldots \ldots . \ldots . \ldots \ldots$

Table (2.7-2). Drag coefficient correlations for non-spherical particles ....................51

Table (2.8-1). A brief literature review of modeling of mixing processes in solid -liquid mixing

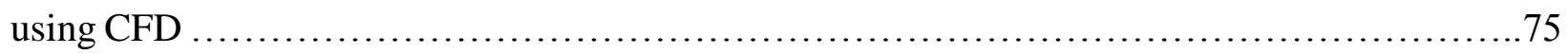

Table (3.2-1). Specifications of glass beads ........................................ 84

Table (3.3-1). Parameters for ERT measurements ...................................... 87

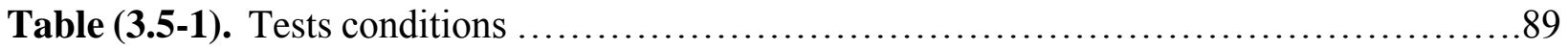

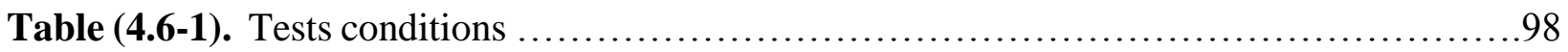




\section{List of Figures}

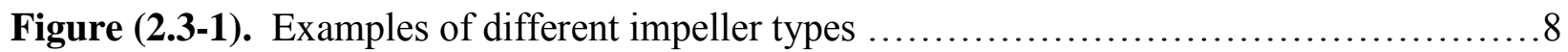

Figure (2.6-1). Classification of solid concentration measurement techniques .................31

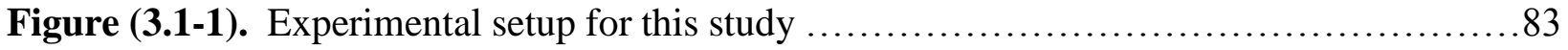

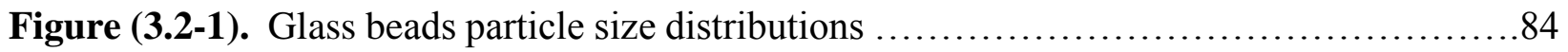

Figure (4.4-1). Grid independency: Axial velocity profiles at $1.5 \mathrm{~cm}$ below the Maxblend impeller $\left(N=480 \mathrm{rpm}, X=30 \mathrm{wt} \%, d_{p}=209 \mu \mathrm{m}, C=T / 8\right)$

Figure (4.4-2). Grid independency: Solid concentration profiles at $1.5 \mathrm{~cm}$ below the Maxblend

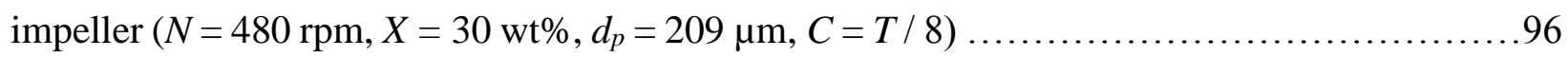

Figure (4.5-1). Scaled residuals for complete glass beads suspension ......................97

Figure (5.1-1). Conductivity tomograms acquired for the particle suspensions agitated at two stirrer speeds (Maxblend impeller, $C=T / 8, X=20 \mathrm{wt} \%$, and $d_{p}=752 \mu \mathrm{m}$ ) $\ldots \ldots \ldots \ldots \ldots \ldots \ldots . \ldots 100$

Figure (5.1-2). Dimensionless mean conductivity (Maxblend impeller, $C=T / 8, X=10 \%, d_{p}=$ $209 \mu \mathrm{m}$, and $N=180 \mathrm{rpm})$

Figure (5.1-3). Solid particle distributions at three different agitation speeds: (a) vertical sliced images, and (b) 3D images (Maxblend impeller, $C=T / 8, X=10 \%$, and $d_{p}=537 \mu \mathrm{m}$ ) 102

Figure (5.1-4). 3D image developed by slicer-dicer for Maxblend impeller $(d p=209 \mu \mathrm{m}, X=$ $20 \%, C=T / 8, N=300 \mathrm{rpm})$ 103

Figure (5.1-5). Axial particle concentration profile at different agitation speeds (Maxblend Impeller, $C=T / 8, X=10 \mathrm{wt} \%$, and $\left.d_{p}=537 \mu \mathrm{m}\right)$

Figure (5.1-6). Effect of the agitation speed on the degree of homogeneity attained with baffled and unbaffled Maxblend impeller $(\mathrm{C}=T / 8, X=10 \mathrm{wt} \%$, and $d p=537 \mu \mathrm{m})$

Figure (5.1-7). Effect of power on homogeneity for the baffled and unbaffled Maxblend impeller $\left(C=T / 8, X=10 \mathrm{wt} \%, d_{p}=752 \mu \mathrm{m}\right)$ .106 
Figure (5.1-8). Estimation of just-suspended speed using the impeller power (Maxblend Impeller, $\left.C=T / 8, X=10 \%, d_{p}=209 \mu \mathrm{m}\right)$

Figure (5.1-9). Effect of the particle concentration on the level of homogeneity at different agitation speeds (Maxblend Impeller, $C=T / 8$, and $d_{p}=752 \mu \mathrm{m}$, Max. std. dev. $=1.38 \%$ )

Figure (5.1-10). Effect of the impeller speed on mixing indices at different solid loadings (Maxblend impeller, $C=T / 8, d_{p}=209 \mu \mathrm{m}$, Max. std. dev. $=1.29 \%$ ) 111

Figure (5.1-11). Influence of the particle diameter on the degree of homogeneity at different agitation speeds (Maxblend Impeller, $C=T / 6, X=10 \mathrm{wt} \%$, Max. std. dev. $=1.09 \%$ ) ....

Figure (5.1-12). Effect of the power consumption on homogeneity for different types of impellers $\left(C=T / 8, X=10 \mathrm{wt} \%, d_{p}=752 \mu \mathrm{m}\right)$

Figure (5.1-13). Power required for different impellers to attain maximum homogeneity $(C=T / 6$, $X=10 \mathrm{wt} \%$, and $\left.d_{p}=209 \mu \mathrm{m}\right)$

Figure (5.1-14). Effect of the stirrer off-bottom clearance on the level of homogeneity at various agitation speeds (Maxblend Impeller, $X=$ wt $20 \%$ and $d_{p}=209 \mu \mathrm{m}$, Max. std. dev. $=1.03 \%$ ) $\ldots .116$

Figure (5.2-1a). (a) Conductivity tomograms, and (b) particle concentration contours created by CFD for four different horizontal planes (Maxblend impeller, $d_{p}=209 \mu \mathrm{m}, X=10 \mathrm{wt} \%, C=T / 8$, and $N=420 \mathrm{rpm})$. 118

Figure (5.2-1b). (a) Conductivity tomograms, and (b) particle concentration contours created by CFD for four different horizontal planes (Maxblend impeller, $d_{p}=752 \mu \mathrm{m}, X=20 \mathrm{wt} \%, C=T / 8$, and $N=240 \mathrm{rpm})$

Figure (5.2-2). Stirrer torque versus agitation speed for the Maxblend impeller $\left(d_{p}=209 \mu \mathrm{m}, X\right.$ $=10 \mathrm{wt} \%$, and $C=T / 8)$

Figure (5.2-3). Estimation of just suspension speed using averaged solid volume fraction versus agitation speed for the Maxblend impeller $\left(d_{p}=209 \mu \mathrm{m}, X=10 \mathrm{wt} \%\right.$, and $\left.C=T / 8\right)$

Figure (5.2-4). Influence of agitation speed on extent of homogeneity for the Maxblend impeller $\left(d_{p}=209 \mu \mathrm{m}, X=10 \mathrm{wt} \%\right.$, and $\left.C=T / 8\right)$ 
Figure (5.2-5). Particle concentration contours created by CFD at various agitation speeds for the Maxblend impeller $\left(d_{p}=209 \mu \mathrm{m}, X=10 \mathrm{wt} \%, C=T / 8\right)$ : (a) $N=120$, (b) $N=150$. (c) $N=420$, and (d) $N=480 \mathrm{rpm}$

Figure (5.2-6). Employing CFD to create particle concentration contours for four different horizontal planes at various agitation speeds $\left(d_{p}=209 \mu \mathrm{m}, X=10 \mathrm{wt} \%\right.$, and $\left.C=T / 8\right)$ : (a) $N=$ 180 , (b) $N=240$, and (c) $N=360 \mathrm{rpm}$

Figure (5.2-7). Streamlines of the solid particles for the Maxblend impeller $\left(\left(d_{p}=209 \mu \mathrm{m}, C=\right.\right.$ $T / 8, X=10 \mathrm{wt} \%$, and $N=420 \mathrm{rpm})$

Figure (5.2-8). Axial particle concentration profile calculated using CFD for the Maxblend impeller $\left(d_{p}=209 \mu \mathrm{m}, X=10 \mathrm{wt} \%\right.$, and $\left.C=T / 8\right)$ .125

Figure (5.2-9). Clouding height for the Maxblend impeller measured ( $d_{p}=209 \mu \mathrm{m}, X=10 \mathrm{wt} \%$, $C=T / 8$ ): (a) $N=150$, and (b) $N=420 \mathrm{rpm}$

Figure (5.2-10). Normalized clouding height at different agitation speeds for the Maxblend impeller $\left(d_{p}=209 \mu \mathrm{m}, X=10 \mathrm{wt} \%\right.$, and $\left.C=T / 8\right)$.

Figure (5.2-11). Influence of baffles on level of homogeneity at different agitation speeds for the Maxblend impeller $\left(d_{p}=209 \mu \mathrm{m}, X=10 \mathrm{wt} \%\right.$, and $\left.C=T / 8\right)$ 128

Figure (5.2-12). (a) Particle concentration contours at a vertical plane, and (b) particle concentration contours at different horizontal planes for the Maxblend impeller without baffles $\left(d_{p}\right.$ $=209 \mu \mathrm{m}, X=30 \mathrm{wt} \%, C=T / 8$, and $N=420 \mathrm{rpm})$ .128

Figure (5.2-13). Velocity vectors for solid particles for the Maxblend impeller $\left(d_{p}=209 \mu \mathrm{m}, X=\right.$ $10 \mathrm{wt} \%, C=T / 8$, and $N=480 \mathrm{rpm})$

Figure (5.2-14). Impact of agitation speed on mixing index for the Maxblend impeller $\left(d_{p}=209\right.$ $\mu \mathrm{m}, C=T / 8$, and $X=10 \mathrm{wt} \%)$.

Figure (5.2-15). Influence of the solid particle diameter on the extent of homogeneity for the Maxblend impeller $(C=T / 8, X=10 \mathrm{wt} \%$, and $N=300 \mathrm{rpm})$

Figure (5.2-16). Particle concentration contours for the Maxblend impeller $(C=T / 8, X=10 \mathrm{wt} \%$, and $N=300 \mathrm{rpm}$ ): (a) $d_{p}=111$, and (b) $d_{p}=752 \mu \mathrm{m}$ 132 
Figure (5.2-17). Extent of homogeneity versus particle specific gravity for the Maxblend impeller $\left(d_{p}=209 \mu \mathrm{m}, C=T / 8, X=10 \mathrm{wt} \%\right.$, and $\left.N=300 \mathrm{rpm}\right)$

Figure (5.2-18). Particle concentration contours for the Maxblend impeller $\left(d_{p}=209 \mu \mathrm{m}, C=\right.$ $T / 8, X=10 \mathrm{wt} \%$, and $N=300 \mathrm{rpm}$ ): (a) $\mathrm{SG}=1.5$, and (b) $\mathrm{SG}=5$ 134

Figure (5.2-19). Influence of the solid loadings on the extent of homogeneity for the Maxblend impeller $\left(d_{p}=209 \mu \mathrm{m}, C=T / 8\right.$, and $\left.N=480 \mathrm{rpm}\right)$ 135 


\section{Chapter 1}

\section{INTRODUCTION}

It is generally accepted that mixing processes play a vital role for commercial success of a process industry because of extensive use of mixing in various unit operations. Solid-liquid mixing operation is one of the most pivotal mixing operations because of its wide range of utilizations in several unit processes and operations. Due to complex behaviour of fluid flow and various factors affecting solid-liquid mixing, it is very difficult to predict optimum criteria to achieve the desired results. It should be noted that the level and degree of mixing in a mixing tank depends significantly on various design parameters of the tank and the impeller. Mechanically stirred tanks are employed for various purposes such as maximizing homogeneousness of the system taking concentration gradients into considerations (Tatterson, 1991), and improving mass transfer among the different phases. Sometimes understanding the parameters affecting the mixing processes can be complicated due to the number of variables involved (Tatterson, 1991). The main goals of mixing operation in solid-liquid system are (Mak, 1992): (a) to prevent settling of particles in an agitated vessel, (b) to improve the mass transfer between two phases by increasing surface areas for the contact between the liquid and solid particles, and (c) to ensure the uniform dispersion and distribution of particles throughout the mixing tank.

Several factors such as the quality and quantity of products, mixing time, power consumption, and cost effectiveness need to be taken into considerations while designing the mixing vessel and setting the operating parameters. Some research works have been reported in the literature to quantify the particle concentration and distribution inside the mixing vessels by experimental investigations in terms of just suspension speed (Zwietering, 1958; Yoshida et al., 2012), relative standard deviation (RSD) (Magelli et al., 1991; Barresi and Baldi, 1987), and clouding height (Bittorf and Kresta, 2003; Sardeshpande et al., 2009). Some researchers have employed the invasive (Sharma and Das, 1980; Bamberger and Greenwood, 2004; Shan et al., 2008) and noninvasive (Guillard et al., 2000; Bolton and Primrose, 2005; Ren et al., 2008; Rodgers et al., 2009) 
particle concentration measurement techniques to assess the local mixing quality in solid suspensions. All these invasive techniques have the problem of having the presence of probe inside the agitated tank, which may affect the flow pattern of the local field. Electrical resistance tomography (ERT) is a simple, robust and non-invasive flow visualisation technique for assessing the local mixing quality by taking the conductivity variations into account. Various research works have been reported in the literature to assess the local mixing quality using ERT (Hosseini et al., 2010a; Tahvildarian et al., 2011; Harrison et al., 2012; Carletti et al., 2014). A review on ERT major applications in chemical engineering has been reported by Sharifi and Young (2013). Due to the complex nature and various factors affecting solid-liquid mixing, sometimes it is impractical, time consuming, and expensive to examine and analyze the mixing quality in solid suspensions using these experimental methods.

Computational fluid dynamics (CFD) has evolved as an efficient and effective tool to get detailed information of complex fluid flow in less time and resources (Kazemzadeh et al., 2016; Fathi Roudsari et al., 2016). This technique can be utilized to retrieve detailed information about velocity and concentration profiles in the mixing tanks. Although CFD cannot replace experimental measurements entirely, it can substantially reduce the number of experimental measurements and costs involved. CFD has been employed by some researchers to quantify the solid particles distribution in terms of just suspension speed (Kee and Tan, 2002; Murthy et al., 2007; Srinivasa and Jayanti, 2007; Panneerselvam et al., 2008) and clouding height (Micale et al., 2004; Ochieng and Lewis, 2006; Sardeshpande and Ranade, 2012). Some researchers have utilized CFD to examine the effect of solid loading (Altway et al., 2001; Oshinowo and Bakker, 2002), stirrer type (Khopkar et al., 2006; Cokljat et al., 2006), particle shape (Fan et al., 2005; Scully and Frawley, 2011), and particle size (Sha et al., 2001; Montante et al., 2001) in solid-liquid mixing operations. Hosseini et al. (2010b) employed CFD to compare the mixing performances of the A100, A200, and A310 axial-flow impellers. Tamburini et al. (2013) utilized CFD to analyze the solid particles distribution in fully baffled mixing vessels furnished with the Rushton turbine (a radial-flow impeller).

After a thorough literature review, it is necessary to mention that no comprehensive research work has been reported regarding the assessment of the local solid concentrations and distributions in a slurry reactor furnished by a Maxblend impeller. The major goal of this research work was to 
assess the mixing performance of a Maxblend impeller in a slurry reactor using the ERT and CFD techniques. The Maxblend impeller is one of the most efficient types of the new generation of the close clearance impellers with a unique geometry. The research objectives of this study were: (a) to quantify solid suspensions in a mixing vessel through just suspended speed, clouding height and homogeneity approaches, (b) to examine the impacts of various geometrical configurations, design parameters and operating conditions on the local mixing quality in a slurry reactor, (c) to analyze the physical characteristics of the solid particles such as specific gravity and average particle size on the mixing quality for solid-liquid operation, and (d) to compare the mixing performance of the Maxblend impeller for liquid-solid mixing operation to those measured for the A200 (an axialflow impeller) and the Rushton turbine (a radial-flow impeller).

Chapter 2 is divided into two sections: the first section is devoted to the thorough literature review on solid-liquid mixing. The second section provides the objectives for this research work.

Chapter 3 provides the detailed information about the experimental setup, procedures and tests conditions employed in this study.

Chapter 4 explains in details the development of computational fluid dynamics (CFD) model utilized in this work.

Chapter 5 provides the ERT and CFD results with the comprehensive discussions. This chapter is organized in two sections:

- Section 5.1 provides the assessment of the mixing performance of a Maxblend impeller in a slurry reactor through ERT.

- Section 5.2 explains the mixing efficiency of a Maxblend impeller for solid-liquid mixing operation using CFD.

Chapter 6 provides overall conclusions and recommendations for the future research work. 


\section{Chapter 2}

\section{LITERATURE REVIEW}

\subsection{Solid-liquid Mixing}

Mixing essentially plays a pivotal part in chemical and process industries in achieving desired products. Mixing is regarded as a process which maximises the homogeneousness of a system. In other words, mixing ideally is degree of equilibrium at which all the elements in the mixing vessel are distributed uniformly throughout the system taking concentration gradients into considerations (Tatterson, 1991). Solid-liquid mixing operation is one of the most pivotal mixing operations because of its wide range of utilizations in several unit processes and operations (Mak, 1992). A survey result presented in a workshop in 1989 by Mixing 3A group of AIChE in England concluded that approximately $80 \%$ of products (chemical) available utilized solid-liquid mixing.

In solid-liquid mixing operations, particles generally tend to accumulate at the base of the mixing tank because in most of the cases, density of solids is higher in comparison to that of liquid. An external force is required to lift up solid particles into the liquid and disperse them throughout the system. In case of solid-liquid mixing, the agitation achieved by the impeller rotation provides the required force. Combined effects of (a) the lift and drag forces exerted by the flowing fluid and (b) the power dissipated by turbulent eddies in the bulk fluid flow are responsible for lifting up the solid particles and distributing them throughout the system (Jafari et al., 2012). 


\subsection{Applications}

Mixing operations are prevalent in several industries due to their extensive applications. Paul et al. (2004) identified those industries as fine chemicals, pharmaceuticals, agrichemicals, petrochemicals, paints, consumer goods, food processing, water treatment, paper and pulp and minerals processing.

Solid-liquid mixing is an extremely significant mixing operation due to its wide range of applications in several unit operations (Mak, 1992). They are: (a) solid particles dispersion (b) leaching and dissolution (c) crystallization (d) precipitation (e) adsorption and desorption (f) solid catalyzed reactions (g) ion-exchange (h) suspensions polymerization, and (i) an activated sludge process.

Minerals processing makes use of solid-liquid mixing operations in various stages, thus, making it one of the major fields of solid-liquid mixing applications (Ayranci et al., 2010).For efficient interactions of solids and liquids, solid-liquid mixing finds great importance in mineral processing as the slurries contain high solids loading with different mineral compositions. The concrete production industry is another area where mixing plays a vital role (Bjegovic et al., 1995). Guo et al. (2014) studied effects of solid-liquid mixing on microstructure of semi solid A356 aluminium alloy.

The suspension of solids in liquid medium in an agitated mixing tank is often encountered in leaching and reactions carried out in presence of a solid catalyst (Baldi et al., 1978). The agitated tank is the most common reactor for polymerization reactions thus highlights the significance of mixing (Gerstenberg et al., 1983). The processing operating conditions of solid-liquid mixing in a slurry reactor was investigated by Nasr-el-din et al. (1996). They explained in details the applications of solid-liquid mixing in process industries. Mixing and dissolution phenomena of pharmaceutical materials in bulk fluid flow of agitated vessels were investigated by Hormann et al. (2011). Solid-liquid loop reactor which has significant advantages and useful in polymerization was studied by Liang et al. (1996). Liquid/solid fluidized beds which are used for many operations like crystallization, sedimentation and catalytic cracking are extremely useful in petrochemical, minerals , food processing and paper industries (Aghajani et al., 2005). 
Information on solids dispersion and distribution in solid-liquid mixing operations in mechanically agitated tanks is extremely significant in crystallization process. The impact of solid particles on micro-mixing of solid-liquid system in an agitated tank which affects the quality of final products in several industrial processes such as crystallization, precipitation, mineral processing and bio-chemical reactors was studied by (Yang et al., 2012). Novel staged continuous crystallizer which could be useful in pharmaceutical industries was proposed by Yu et al. (2009) taking solid-liquid mixing into considerations. Solid particles suspension in unbaffled vessel which provides great advantages in vast range of utilizations such as food, crystallisation and pharmaceutical processes, was investigated by Brucato et al. (2010).

Khare et al. (1990) investigated two-phase mixing characteristics in a bubble column slurry reactor which is widely employed in industries because of its simple geometric configuration and ease of operations. Coaxial mixers could be very useful in the preparation of very complex formulation and solids suspension prevalent in several industrial processes such as paper and pulp, cosmetics, food etc. (Foucault et al., 2004). Cullen (2009) explained the importance of solid-liquid mixing in maximizing the homogeneity of the system in food industries.

Mixing and two phase mass transport phenomenon in a novel aerobic immobilized cell bioreactor were studied by Kodialbail and Sriniketan (2011). The interactions between turbulent flow field and discrete particles have great significance in bio-chemical processes (Caulet et al.,1996).The macromixing of solid and liquid phases in a dry anaerobic digester was studied by Benbelkacem et al. (2013). Double- shell tank system for handling wastes makes use of effective solid-liquid mixing (Wells et al., 2011).

Adsorption is extremely important in various industrial processes particularly in lubrication, detergency and catalysis. The mixing and adsorption behaviour of alkanes and ethers at the interface of solid-liquid media was investigated by Duim and Clarke (2006). Adsorption/desorption isotherms are most commonly used to assess surface properties of particles in suspension (Shrotri et al., 1998). van der Wielen et al. (1998) studied transport of pulsed adsorbent in an adsorptive reactor operated on countercurrent mode. 


\subsection{Geometric Design and Considerations in Mixing}

It is imperative to mention that the level and degree of mixing in mixing tank depends significantly on various design parameters of the tank and the impeller. The influence of the vessel geometry is paramount in mixing quality and power input. The following variables greatly affect the optimum mixing in solid-liquid mixing operations:

1. Mixing tank: The shape and size of the tank is an essential parameter which significantly influences the hydrodynamic flow behaviour of fluid, and as a result, yield and quality of mixing (Paul et al., 2004). There are different shapes of tank reported in literature such as rectangular, cylindrical, and square. The shape of the bottom head of the tank such as flat, conical or dished is crucial for the optimal mixing. The selection of shape of the tank depends on the desired results. However, cylindrical tanks with dished bottom heads are generally preferred. For instance, a tank with flat bottom draws 10 to $20 \%$ additional power than that with a dished bottom to attain high degree of homogeneity (Bittorf and Kresta, 2003). The number of impellers required for mixing greatly depend on liquid height to vessel diameter ratio $(h / T)$. For $h / T=1$, one impeller is sufficient for perfect mixing. However, more than one impeller must be used when $h / T$ is greater than one for top entry impeller.

2. Shaft: The shaft can be installed in the vessel from the top, bottom or side depending on the desired results. Side entering shaft requires sealing to avoid leakages whereas top entering one does not need it. The shaft with side entry gives better results for solid particles with low settling velocity (Tattereson, 1994).

3. Impeller: Impeller provides necessary force required to move up solid particles from the base and disperse them in the mixing tank. Impellers are broadly classified into two categories based on the flow pattern. They are: (a) axial (down and up) flow impellers and (b) radial (side to side) flow impellers. Axial (down and up) impellers are preferred over radial (side to side) impellers for solid liquid mixing to achieve better results (Paul et al., 2004). Radial flow impellers are very susceptible to viscosity of the media. It is important to mention about the Maxblend impeller which is to be considered for this investigation of solid-liquid mixing operations. It is a new generation

of closed clearance impeller which has a unique geometry. The performance of this impeller for 
dispersion and distribution of solid particles in solids suspensions is a matter of great curiosity. Examples of different impeller types are shown in Figure (2.3-1).

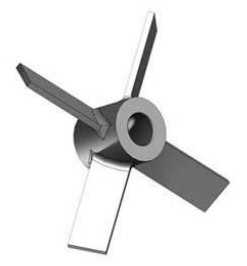

Pitched blade turbine

(Axial impeller)

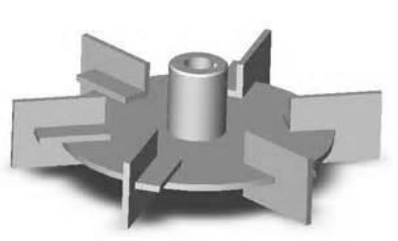

Rushton

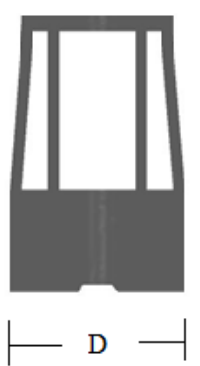

Maxblend impeller

(Radial impeller)

Figure (2.3-1). Examples of different impeller types.

To generate axial flow with low shear rate, hydrofoil impellers (A310 or A315) are preferred. Helical ribbons, anchor and screw impellers are used to produce creeping flows.

4. Baffle: Baffles are basically installed to prevent vortex and dead zones formation in the mixing tank. Baffles are not needed for laminar flow conditions. Baffles (flat blade) with a width of $T / 12$ - T/10 are reported to be effective for particles suspension (Lu et al., 1997; Paul et al., 2004). There should be a wall clearance (T/72) behind the baffles to prevent formation of dead zones. Baffles are necessary to produce controlled vortex for suspension and dispersion of floating solids (Joosten et al., 1977; Siddiqui, 1993).

5. Others: A variable frequency drive to control and maintain impeller speed and a torque meter to measure torque are recommended for effective and efficient operations. 


\subsection{Characterisation of Solid Distribution}

The level and state of suspension is very critical in solid-liquid mixing operation. It can be characterised broadly in three different levels (states) (Paul et al., 2004). They are as follows:

a) On-bottom or partial suspension: Some of the particles rest at the base of the mixing tank because the agitation provided by the stirrer is not sufficient enough to lift up all particles from the bottom of the tank. In this state, surface areas of all the solid particles are not available to the liquid for effective mass/heat transfer and chemical reactions. Although this suspension does not provide sufficient conditions for many unit operations, dissolution of very soluble solid particles can take place at this state.

b) Off-bottom (complete) suspension: In this state, the agitation provided by the impeller is sufficient enough to lift up all the solid particles from the base of the mixing vessel. No solid particle stays at the base of the vessel for more than 1 to 2 seconds. The stirrer speed which causes complete suspension is known as just suspended speed $\left(N_{j s}\right)$ and the condition itself is referred as Zwietering criterion. Although maximum surface areas of solid particles are available for mass or heat transfer and the chemical reaction, uniform distribution of solid particles may not be achieved in the system. At this state, distinct interface exists between slurry suspensions and clear liquid layer. Further increase of the agitation speed beyond just suspended speed increases the suspended region and reduces the clear liquid layer. The slurry height criterion proposed by Kraume (1992) to evaluate just suspended speed is achieved, once the height of the slurry reaches approximately $90 \%$ of the liquid level and the rest $10 \%$ being comprised of clear liquid region.

c) Uniform suspension: In this state, the agitation is sufficient enough to suspend all solid particles uniformly throughout the mixing tank. Uniform suspension is mostly observed in solid catalyzed reactions and crystallization. In this suspension, further increment in stirrer speed may not increase the distribution of solid particles in the system (Paul et al., 2004). At this state, agitation speed of impeller is known as critical impeller speed for uniform suspension. Uniform suspension is not always desirable in several unit operations. 


\subsubsection{Models and Methods for Characterizing Just Suspension Speed $\left(N_{j s}\right)$}

The first successful investigation and study available in literature on solid-liquid mixing operations was carried out by Zwietering (1958). He proposed an empirical correlation by using visual observations to obtain a large set of experimental data. He then performed dimensional analysis of experimental results. The equation developed by him is given by:

$$
N_{j s}=S\left(\frac{g \Delta \rho}{\rho_{L}}\right)^{0.45}\left(\frac{X^{0.13} d_{p}^{0.2} v^{0.1}}{D^{0.85}}\right)
$$

where $S$ is dimensionless number and remains constant for a system geometry and $\Delta \rho=\rho_{S}-\rho_{L}$. $g, \rho_{s}, \rho_{L}, v, d_{p}, D$, and $X$ are acceleration due to gravity, density of solid particle, density of liquid, kinematic viscosity, diameter of particle, stirrer diameter and solid weight fraction, respectively. Although this method is simple and non-intrusive, it is not applicable for dense solid suspensions and opaque systems. As this method uses an empirical correlation, it should not be used for a system outside its test range (solid loading $<15 \%$, particle size $=0.2-1 \mathrm{~mm}$, vessel diameter $=$ $0.15-0.6 \mathrm{~m}$, density of liquid $=790-1600 \mathrm{Kg} / \mathrm{m}^{3}$ )

Mersmann et al. (1998) proposed a theoretical method to characterise $N_{j s}$ based on power dissipation by two phenomena: (a) power consumption to avoid accumulation and (b) power consumption to disperse solid particles in the bulk fluid flow. The agitation speed $N$ that is critical to prevent solid accumulation can be expressed in terms of a constant adjusted Froude number $\left(\mathrm{F}_{\mathrm{r}}^{*}\right)$;

$$
F_{r}^{*}=\frac{N^{2} D^{2} \rho_{L}}{d_{p} \Delta \rho g}=\text { Constant }
$$

Hence, $\quad N_{j s} \propto\left(\frac{g \Delta \rho}{\rho_{L}}\right)^{0.5} d_{p}^{0.5} D^{-1}$ 
where $\Delta \rho=\rho_{s}-\rho_{L}, D, d_{p}, g, \rho_{s}$, and $\rho_{L}$ are impeller diameter, diameter of particle, gravity, density of particle and density of liquid, respectively. As values for $N_{j s}$ evaluated by this method are not based on experimental data. Mersmann et al. (1998) later proposed an experimental method to verify the equation obtained by theoretical method. They visually observed and measured the slurry height with respect to the height of the fluid.

Rewatkar et al. (1991) proposed an experimental method based on variation of power consumption by impeller with increase in the amount of particles suspended, change in continuous (liquid) phase mixing time and decrease in count rate assessed from radioactive tracer. This method is non-intrusive and can be applicable for dense or opaque systems. But this method requires accurate and reliable assessment of power consumption and mixing time. The criteria to explain particle distribution in the mixing tank are not clear.

Musil et al. (1984) came forward with a method based on variation in particle concentration close to the tank base with increase in impeller speed. This method can be used for opaque system. Accurate and perfect measurement of particle concentration in the system is very difficult due to its intrusive nature.

Chapman et al. (1983) proposed a method by taking measurements of peak values in particle concentration near the tank base with increment in stirrer speed. This technique can be applicable to the dense and opaque systems. Accurate and reliable measurement of particle concentrations is highly challenging due to its invasive nature.

Micale et al. (2000) put forward a method based on variation in the pressure, measured at the tank base with increase in impeller speed. This method is non-intrusive and independent of material. Method put forward by them to rule out dynamic pressure head effect is not convincing. Proper and careful selection of pressure recording device is crucial.

Kolar (1961) put forward a method which assumes that the energy required for mixing at critical conditions in the system is correlated to the potential energy of the solid particles. The settling velocity of particles is proportional to tip speed of the impeller. But it should be noted that the settling velocity of solid particles in turbulent flow fluid is different from that observed in stationary fluid. Although this model is simple, it cannot predict just suspension speed $\left(N_{j s}\right)$ precisely. 
Baldi et al. (1978) proposed that the particles suspension is caused by the turbulent eddies of the size almost equal to the diameter of solid particle. The power dissipated by the turbulent eddies to the solid particles is responsible to move them up to a height in order of particle diameter. Baldi gave the following co-relation for just suspension speed $\left(N_{j s}\right)$ :

$$
N_{j s} \propto v^{0.17}\left(\frac{g \Delta \rho}{\rho_{L}}\right)^{0.42} X^{0.125} d_{p}^{0.14} D^{-0.89}
$$

Where $\Delta \rho=\rho_{s}-\rho_{L} . D, X, g, v, \rho_{s}, \rho_{L}, d_{p}$, are impeller diameter, weight fraction, gravity, kinematic viscosity, particle density, liquid density and particle diameter respectively. This method is not able to describe satisfactorily that the impeller which causes mass circulation is more effective for solids suspension than the impeller which generates excessive turbulence. The assumption that particles are solely suspended by turbulence as proposed by this model casts doubt.

Narayanan et al. (1969) developed a model to predict $N_{j s}$ with an assumption that no slip condition exists between solid particle and liquid. They observed how vertical forces balance themselves while acting on a solid particle. It was developed for very dilute solids suspension. The approach adopted by authors does not explain distribution of particles in an agitated tank precisely.

Subbarao and Taneja (1979) developed a method by equilibrating settling velocity of particles with bulk fluid velocity in an agitated mixing tank. In this model, they used a correlation between fluid velocity and porosity of a fluidised bed to determine settling velocity of solid particles. Although this method is very simple, it does not describe the distribution of particles precisely because authors did not consider effects of viscosity and solid concentration.

Ditl and Rieger (1985) developed a model based on the same turbulence concept as proposed by Baldi et al. (1978) that different sizes of turbulent eddies are responsible for lifting up solid particles in an agitated vessel. Although these two models are based on similar concepts, Baldi et al. (1978) introduced empirical reasoning by using experimental data to set exponents of particle diameter and solid concentration where as Ditl and Rieger (1985) did not employ any experimental data to adjust their developed model. 
Musil and Vlk (1978) proposed a theory by equilibrating kinetic energy of solid particles and moving fluid. Their concept is almost similar to initial assumption adopted by Kolar (1961). They expressed their results in terms of a critical Reynolds number that is correlated to particle Reynolds number $\left(R_{e p}\right)$ and Archimedes number (Ar). The approach adopted by Musil and Vlk (1978) was completely disapproved by Ditl and Rieger (1985) due to physical assumptions and mathematical errors associated with their theory.

Shamlou and Zolfagharian (1987) developed a model to estimate the critical and necessary conditions required for incipient motion of solid particles by equilibrating hydrodynamical forces of gravity, drag, lift and buoyancy acting on particles settling at the vessel base with average acceleration of the liquid close to the base of the mixing vessel. The authors predicted just suspended speed $\left(N_{j s}\right)$ as follows:

$$
N_{j s}=A N_{p}^{-1 / 3}\left(\frac{g \Delta \rho}{\rho_{L}}\right)^{1 / 2} d_{p}^{1 / 6} c_{v}^{1 / 3} T D^{-5 / 3}
$$

where $A$ is constant and $\Delta \rho=\rho_{s}-\rho_{L} . N_{p}, D, T, \rho_{s}, \rho_{L}, d_{p}, C_{V}$ and $g$ are power number, impeller diameter, tank diameter, density of solid particle, density of liquid, diameter of particle, volume fraction of solid and gravity respectively. Although this model does not require any experimental adjustments, the various factors influencing solid particles distribution is not reported precisely.

Molerus and Latzel (1987a, 1987b) proposed a model based on the concept that particles suspension in a stirred tank is influenced by two different mechanisms for coarse and fine particles (expressed in terms of Archimedes number). The first mechanism defines and explains the complete suspensions of very fine particles $(\mathrm{Ar} \leq 40)$ being achieved at adequately high shear stress in the vicinity of wall boundary layers of the tank. The second mechanism, befitting only to coarse particles (Ar $>40)$ is primarily depended upon the pump characteristics of an agitated vessel.

For fine particles $(\mathrm{Ar} \leq 40)$, 


$$
N_{j s} \propto\left(\frac{g d_{p} \Delta \rho}{\rho_{L}}\right)^{0.56} v^{-0.11} D^{-1} T^{0.11}
$$

For coarse particles $(\mathrm{Ar}>40)$,

$$
N_{j s} \propto(g \Delta \rho)^{0.5}\left(\frac{d_{p}}{\rho_{L}}\right)^{0.14} D^{-1}\left(C_{v} h\right)^{0.36}
$$

where $\Delta \rho=\rho_{s}-\rho_{L} \cdot N_{j s}, g, \rho_{s}, \rho_{L}, d_{p}, D, C_{V}$, and $h$ are just suspension speed, gravity, solid particle density, liquid density, particle ( solid) diameter, impeller diameter, volume fraction of solid and height of slurry, respectively. This model needs an accurate and precise correlation to assess shear rates at the wall boundary layer of the tank.

Wichterle (1988) proposed a theoretical model for particles suspension measuring difference in terminal settling velocity of solid particle and liquid velocity. The ratio between just suspension speed $\left(N_{j s}\right)$ and settling velocity allows to evaluate $N_{j s}$. The scale up rule suggested for impeller diameter on just suspended speed is not convincing.

Yoshida et al. (2012) employed Zwietering's visual method to predict the values of $N_{j s .}$ They made use of a forward and reverse rotating stirrer at unsteady state to eliminate the effect of air vortex formation. They proposed a correlation for predicting $N_{j s}$ as follows:

$$
N_{j s}=1.20 B_{o}^{0.1} d_{p}^{0.2}\left(\frac{g \Delta \rho}{\rho_{L}}\right)^{0.5}\left(\frac{D}{T}\right)^{-1.4}\left(\frac{C}{T}\right)^{0.7} D^{-0.7}
$$

For $1 / 10<C / T<1 / 6$, 


$$
N_{j s}=0.679 B_{o}^{0.1} d_{p}^{0.2}\left(\frac{g \Delta \rho}{\rho_{L}}\right)^{0.5}\left(\frac{D}{T}\right)^{-1.4}\left(\frac{C}{T}\right)^{0.4} D^{-0.7}
$$

For $1 / 6<C / T<1 / 3$

where $\Delta \rho=\rho_{s}-\rho_{L}, N_{j s}, B_{o}, \rho_{s}, \rho_{L}, d_{p}, D, C$, and $T$ are just suspension speed, particle concentration percent, solid density, liquid density, particle diameter, impeller diameter, impeller clearance and tank diameter, respectively. A brief summary of suspension models is presented in Table (2.4-1). 
Table (2.4-1). A brief summary of suspension models

\begin{tabular}{|c|c|c|c|c|c|c|c|c|c|}
\hline \multirow[t]{2}{*}{ Reference } & \multirow[t]{2}{*}{ Approach } & \multirow[t]{2}{*}{ Status } & \multicolumn{6}{|c|}{ Exponent on } & \multirow[t]{2}{*}{ Remarks } \\
\hline & & & $v$ & $\Delta \rho$ & $\rho_{L}$ & $d_{P}$ & $X$ & $D$ & \\
\hline Zwietering, 1958 & Empirical & - & 0.1 & 0.45 & -0.45 & 0.2 & 0.13 & -0.85 & With dimensional analysis \\
\hline Baldi et al., 1978 & Turbulent & Without adjustment & - & 0.5 & -0.5 & 0.17 & - & -0.67 & $N_{j s} \propto P o^{-0.33}$ \\
\hline "“ & "6 & $\begin{array}{l}\text { Adjusted with } \\
\text { experimental data }\end{array}$ & 0.17 & 0.42 & -0.41 & 0.14 & $\begin{array}{l}0.12 \\
5\end{array}$ & -0.89 & $\begin{array}{l}\text { 1. when } C / D=1 \text {, constant } \\
\text { Po \& } D / T \\
\text { 2. exponent varies with } \\
\text { geometry }\end{array}$ \\
\hline $\begin{array}{l}\text { Mersmann et al., } \\
1985\end{array}$ & Velocity & $\frac{d_{P}}{T}<10^{-3}$ & - & 0.5 & -0.5 & 0.5 & - & -1 & \\
\hline 66 & No clear & $\frac{d_{P}}{T}<10^{-3}$ & - & 0.5 & -0.5 & nil & - & -0.5 & \\
\hline $\begin{array}{l}\text { Shamlou and } \\
\text { Zolfagharian, } \\
1987\end{array}$ & velocity & Theoretical & - & 0.5 & -0.5 & 0.17 & - & -0.67 & $\begin{array}{l}\text { Model confirmed by } \\
\text { experiments } \\
\qquad N_{j s} \propto \mathrm{Po}^{-1 / 3} c_{v}^{1 / 3} T,\end{array}$ \\
\hline $\begin{array}{l}\text { Molerus and } \\
\text { Latzel, } 1987\end{array}$ & $\begin{array}{l}\text { Fluidised } \\
\text { bed }\end{array}$ & $A r<40$ & -0.11 & 0.56 & -0.56 & 0.56 & - & -0.89 & $\begin{array}{l}\text { No concentration effect, } \\
\text { model supplement with } \\
\text { experiments }\end{array}$ \\
\hline “6 & Pump flow & $A r>40$ & - & 0.5 & -0.14 & 0.14 & - & & $N_{j s} \propto c_{v}^{0.36}$ \\
\hline Wichterle 1988 & Velocity & & Funct & on of $A$ & rchime & les $\mathrm{No}$ & & -0.67 & \\
\hline
\end{tabular}




\subsubsection{Clouding Height}

Many research works have been done to develop models to determine the homogeneity by measuring cloud height. For high solids loadings, a clear solid-liquid interface exists above which particle concentration is almost nil. The height (distance) of this interface from the base of the mixing tank is known as clouding height. Particles do not stay above this interface for more than 1 or 2 seconds.

Bittorf and Kresta (2003) proposed a correlation for clouding height for particle distribution at large stirrer diameter and low off-bottom clearance. This model developed by Bittorf and Kresta (2003) is based on two assumptions. First, the mean flow conditions are used to determine the cloud height at higher solids concentration. Turbulent fluctuations in this clear level are considered to be negligible. Second, the constant mean flow velocity at the cloud height can be identified with the help of jet model. The equation is given by:

$$
C_{H}=\frac{N}{N_{j s}}\left[0.84-1.05\left(\frac{C}{T}\right)+0.7 \frac{\left(\frac{D}{T}\right)^{2}}{1-\left(\frac{D}{T}\right)^{2}}\right]
$$

where $C_{H}, N, N_{j s}, C, D$ and $T$ are clouding height, stirrer speed, just suspension speed, off-bottom clearance, stirrer diameter and vessel diameter, respectively.

Bujalski et al. (1999) developed a model to predict cloud height at high solids concentrations (20-40\% solid loading) in flat bottomed tanks. This model is based on wall jets. The types of solids were varied while the tank geometry remained constant. Mixing times in the clear layer can increase up to twenty times longer than the mixing time in the slurry.

Hicks et al. (1996) proposed a model based on wall jets for different types of solids in flat bottomed tanks. They varied the types of solids and experimental configuration but kept the solids concentration at $10 \%$ solid loading. Discrepancies were observed in measuring cloud heights. Mak (1992) developed concentration profiles at high solids concentrations in a tori spherically bottom vessel at three scales of operation. 
Micale et al. (2004) compared and analysed the clouding height obtained from experimental measurements and Eulerian-Eulerian simulations. The simulated results clearly highlighted the impact of particles concentration on the distribution of clouding height. Quantitative comparisons emphasized the requirement of complex inter-phase models to improve and enhance the accuracy and precision of the results obtained from simulations.

Ochieng and Lewis (2006) investigated the cloud height in a fully baffled tank stirred by hydrofoil stirrer. The clouding height was assessed from the axial profiles of particle volume fraction. The approximated clouding height held well with the experimental result up to $10 \%$ particles concentration but inaccuracies increased with increase in particles concentration.

Sardeshpande et al. (2009) observed and assessed the hysteresis in clouding height experimentally that changes with stirrer speed, particularly at high particles concentration. The maximum particles concentration were held at $7 \%(\mathrm{v} / \mathrm{v})$. Such phenomenon could be well obtained by the use of CFD models with reliable and relevant initial assumption, particle-particle interactions and drag correlations.

Clouding height cannot explain local mixing quality accurately because local particle concentration cannot be determined by this approach.

\subsubsection{One Dimensional Sedimentation and Dispersion Model}

Relative standard deviation (RSD) is most commonly used to assess the local mixing quality in multi-phase agitated tanks. RSD is the degree of standard deviation of the local particles concentration with respect to the average particles concentration in the mixing vessel. The value of RSD decreases with increase in homogeneity and becomes zero at perfect homogeneity. RSD is given by:

$$
R S D=\frac{1}{C_{M}}\left[\frac{1}{\left(n_{s}-1\right)} \sum_{i=1}^{n_{s}}\left(C_{i j}-C_{M}\right)^{2}\right]^{1 / 2}
$$


where $C_{i j}, C_{M}$, and $n_{s}$ are local particles concentration at $i^{\text {th }}$ position and $j^{\text {th }}$ speed, mean bulk particles concentration and the number of sampling positions, respectively.

Some researchers used variance $(\sigma)$ to quantify the particles distribution quality. Correlation between variance $(\sigma)$ and RSD is given by:

$$
R S D=\frac{\sigma^{1 / 2}}{C_{M}}
$$

Barressi and Baldi (1987) used one dimensional model assuming the solid phase to be continuum. The local axial mean-time velocity of particles is a sum (vectorial) of the terminal velocity of particles and the liquid velocity. They proposed an adjusted Peclet number $\left(P e^{*}\right)$ to explain the local particle concentrations. By relating the power input in turbulent scale, $P e^{*}$ is given by:

$$
P e^{*} \propto \frac{U_{t o}}{N_{p} N D}
$$

where $P e^{*}, U_{t o}, N_{p}, N$, and $D$ are modified Peclet number, terminal velocity at stagnant medium, power number, stirrer speed and impeller diameter, respectively. They defined a parameter $K$ which is $\frac{1}{P e^{*}}$. Local suspension quality can be obtained in terms of impeller speed by plotting RSD against $K / X^{0.13}$, where $X$ is solid weight fraction.

Magelli et al. (1987, 1991) proposed that inhomogeneity in solids suspension can be quantified by relative standard deviation of the particles concentration with respect to the average concentration value. RSD can be expressed in terms of Peclet number $(P e)$ as: 


$$
R S D=\left[\frac{P e}{2} \frac{e^{2 P e}-1}{\left(e^{P e}-1\right)^{2}}-1\right]^{1 / 2}
$$

and

$$
P e=\frac{U_{t t} h}{D_{e . p}}
$$

where $P e, U_{t t}, h$ and $D_{e . p}$ are Peclet number, terminal velocity at turbulent medium, height of slurry and particle dispersion coefficient respectively.

Shamlou and Koutsakos (1989) proposed a theory by conducting a mass balance on the solid particle over a very thin layer of the liquid in the tank. They assumed that no depletion and/or accumulation of solid particles take place in the system. The authors expressed an equation in terms of dimensionless mode by introducing Peclet number $(\mathrm{Pe})$ :

$$
\frac{U_{t t} d_{p}}{D_{e . p}} \propto \frac{U_{t o}}{N D}
$$

and $\quad P e=\frac{U_{t t} d_{p}}{D_{e . p}}$

where $U_{t t}, U_{t o}, d_{p}, N, D$ and $D_{e . p}$ are terminal velocity at turbulent medium, terminal velocity at stagnant medium, particle diameter, stirrer speed, impeller diameter and particle dispersion coefficient respectively.

Buurman et al. (1985) proposed a modified Froude no. model assuming that not only the small turbulent eddies in the vicinity of inertial sub-field but also the largest eddies play a crucial role in system homogeneity. They expressed an equation as follows: 


$$
N_{R S D} \geq 4.47\left(\frac{g \Delta \rho}{\rho_{L}}\right)^{0.5}\left(\frac{d_{p}}{D}\right)^{0.275} D^{-0.50}
$$

where $\Delta \rho=\rho_{S}-\rho_{L} . N_{R S D}, D, g, \rho_{S}, \rho_{L}$, and $d_{p}$ are stirrer speed at relative standard deviation, impeller diameter, gravity, solid particle density, liquid density and particle diameter respectively.

Penaz et al. (1978) proposed a particle concentration and distribution model based on continuity equation and assumption that turbulence is responsible for molecular diffusion of particle flux. They made use of cylindrical co-ordinates and estimated radial solids distribution also. They also concluded that tangential component of the liquid velocity near the impeller $\left(45^{0}\right.$ pitched blade turbine) region cannot be considered negligible.

Einenkel (1980) assessed the suspension and dispersion pattern of particles (glass beads) in agitated tanks. The outcome of his experiments demonstrated that the particles behaviour in the bulk fluid flow is crucial to explain their distribution. He suggested to use the ratio of terminal settling velocity of an individual solid particle to that of bulk fluid instead of the ratio of solid particle diameter to that of the stirrer diameter. According to author, selection of solid volume fraction over mass fraction is crucial for characterization of solids distribution. The shortcoming of this model is that the mechanism of solid suspension and distribution were not explained clearly and precisely while discussing the results.

Bohnet and Niesmak (1980) developed a model based on one-dimensional dispersion and sedimentation resulting in the function of a Peclet number describing the solids concentration and distribution. The solids concentration is small (up to $6 \%$ solids loading) with no clear interface so that the density of the pure liquid is same as that of the slurry. They reported that the acquirement of the stirrer speed to lift up solid particles is not sufficient condition to ascertain homogeneity in the system. A brief summary of dispersion models is presented in Table (2.4-2). 
Table (2.4-2). A brief summary of dispersion models

\begin{tabular}{|c|c|c|c|c|c|c|c|c|c|}
\hline Reference & $\mathbf{T}(\mathbf{m})$ & Impeller & $\mathbf{C}$ & Conc. & $d_{P}(\mu m)$ & $\rho_{S}\left(K g m^{-3}\right)$ & $\rho_{L}\left(K g m^{-3}\right)$ & Technique & \begin{tabular}{l|l} 
le & Scale-up
\end{tabular} \\
\hline $\begin{array}{l}\text { Barresi and } \\
\text { Baldi, } 1987\end{array}$ & 0.39 & $\begin{array}{l}\text { A } 310, \text { PBT4, DT6, } \\
\text { all T/3 }\end{array}$ & $\mathrm{T} / 3$ & $\begin{array}{l}0.5-5.1 \\
\% \mathrm{Wt}\end{array}$ & $100-500$ & $2600-2670$ & 1000 & sampling & $\mathrm{N} \propto D^{-1}$ \\
\hline & \multicolumn{9}{|c|}{$R S D \propto \frac{U_{t}}{P o^{1 / 3} N D} X^{0.13}$} \\
\hline \multirow[t]{2}{*}{$\begin{array}{l}\text { Buurman et al., } \\
1985\end{array}$} & $0.24-4.26$ & PBT4(T/2.5) & $\mathrm{T} / 3$ & $\begin{array}{l}3-40 \\
\% \mathrm{Vol}\end{array}$ & $157-2200$ & $1200-2650$ & $800-1000$ & sampling & $\mathrm{N} \propto D^{-0.78}$ \\
\hline & \multicolumn{9}{|c|}{$\frac{\rho_{c} N^{2} D^{2}}{g \Delta \rho d_{P}}\left(\frac{d_{P}}{D}\right)^{0.45} \geq 20$ i.e $N_{R S D} \geq 4.47\left(\frac{g \Delta \rho}{\rho_{c}}\right)^{0.5}\left(\frac{d_{P}}{D}\right)^{0.28} D^{-0.5}$} \\
\hline \multirow[t]{2}{*}{$\begin{array}{l}\text { Shamlou and } \\
\text { Koutsakos, } \\
1989 \\
\end{array}$} & 0.225 & PBT6(T/3.5-T/2.3) & vary & $\begin{array}{l}1-6 \% \\
\mathrm{Wt}\end{array}$ & $175-1100$ & $2900-3800$ & 1000 & optical & $\mathrm{N} \propto D^{-1}$ \\
\hline & \multicolumn{9}{|c|}{ Distribution Quality, $\frac{U_{t t} d_{P}}{D_{e, p}} \propto \frac{U_{t}}{N D}, h \neq T$} \\
\hline $\begin{array}{l}\text { Magelli et al., } \\
1991\end{array}$ & 0.236 & A310, PBT2,4 & - & $\begin{array}{l}0.1-0.3 \\
\% \mathrm{Wt}\end{array}$ & $140-980$ & 2450 & $\mathrm{H}_{2} \mathrm{O}, \mathrm{PVP}$ & optical & $\mathrm{N} \propto D^{-0.93}$ \\
\hline
\end{tabular}




\subsubsection{Dispersion and Distribution of Floating Solids}

The models and methods discussed so far for solids suspension and distribution are for those solid particles which have density higher than those of liquid used. Some solid particles which have less density than the liquid, can float on the liquid without adequate application of force. Not much research works have been done involving floating solids.

The critical factor in achieving complete suspension and dispersion for floating solids is associated with the development of controlled vortex in the mixing tank (Joosten et al., 1977; Siddiqui, 1993). The controlled vortex can be achieved by using partially immersed baffles. The energy required for dispersion and distribution of floating solids is more compared to that for settling (sinking) solids.

Joosten et al. (1977) proposed an empirical correlation for suspending floating solids by introducing a Froude number $\left(F_{r}\right)$ for $50 \mathrm{~m}^{3}$ vessel. The equation is given by:

$$
\begin{aligned}
& F_{r}=3.6 \times 10^{-2}\left(\frac{D}{T}\right)^{-3.65}\left(\frac{\rho_{l}-\rho_{s}}{\rho_{l}}\right)^{0.42} \\
& F_{r}=\frac{N^{2} D}{g}
\end{aligned}
$$

where $F_{r}, D, T, N, g, \rho_{l}, \rho_{s}$ are Froude number, impeller diameter, tank diameter, impeller speed, gravity, liquid density and solid density respectively.

Joosten et al. (1977) used $45^{0}$ pitched blade impeller with only one baffle partially submerged in the liquid. Siddiqui (1993) used four baffles to form controlled vortex and reported that his system is more effective than that proposed by Hemrajani et al. (1988) as they did not use any baffles in the tank. 


\subsection{Factors Influencing Solid-liquid Suspension}

Mixing requires a substantial amount of time for processing. It is very essential to know optimum mixing time because over or under mixing can lead to adverse effect on yield (Tatterson, 1991). There are various factors which influence solid-liquid suspensions. They are: (a) physical characteristics of solid and liquid, (b) operating conditions for processing, (c) Geometric configurations and parameters, and (d) Conditions for agitation (Paul et al., 2004). Physical characteristics of solid include particle density, particle size, hardness of solid, particle sphericity, solid wetting characteristics and tendency of solids to entrap air. Physical characteristics of liquid include liquid density, viscosity and difference in density. Operating conditions for processing include height of liquid in the tank, solid loading and solid volume fraction. Geometric parameters comprise of tank diameter, geometry of tank bottom head (flat, cone or dish shaped), stirrer diameter, impeller geometry and type, stirrer clearance, and type and number of baffles. Agitation conditions include stirrer speed, stirrer power, liquid flow pattern and turbulent intensity distribution in the tank. Before discussing effect of various factors on solid suspension, it is necessary to understand settling velocity, hindered settling velocity and particle size distribution.

\subsubsection{Settling Velocity}

Processes involving sinking solids make use of settling velocity, velocity at steady state condition at which the drag force balances the buoyant and gravitational forces without interaction among solid particles. Oldshue (1983) divided the impact of settling velocity into the following three regimes: easy $\left(V_{t}=0.1-0.6 \mathrm{ft} / \mathrm{min}\right)$, moderate $\left(V_{t}=4-8 \mathrm{ft} / \mathrm{min}\right)$, and hard to suspend $\left(V_{t}=16\right.$ $60 \mathrm{ft} / \mathrm{min})$.

Perry and Green (1984) developed an equation to determine the free settling velocity of spherical particles. The equation is given by:

$$
V_{t}=\left[\frac{4 g_{c} d_{p}\left(\rho_{s}-\rho_{l}\right)}{3 C_{D} \rho_{l}}\right]^{1 / 2}
$$


where $V_{t}, g_{c}, d_{p}, \rho_{s}, \rho_{l}$, and $C_{D}$ are terminal velocity, gravitational constant, particle diameter, particle density, liquid density and drag coefficient, respectively. Particle Reynolds number is given by:

$$
R e_{p}=\frac{\rho_{l} V_{t} d_{p}}{\mu}
$$

For the Newtonian turbulent regime $\left(\operatorname{Re}_{\mathrm{p}}>1000\right)$, terminal velocity can be calculated as proposed by Perry and Green (1984):

$$
V_{t}=1.73\left[\frac{g_{c} d_{p}\left(\rho_{s}-\rho_{l}\right)}{\rho_{l}}\right]^{1 / 2}
$$

\subsubsection{Hindered Settling Velocity}

Solid particles concentration greatly influence the degree of homogeneity. The increase of solid loadings, results in, increase of number of particles and viscosity. Hindered settling velocity is developed mainly due to three reasons. They are: (a) interaction among particles, (b) increase of viscosity and density of the slurry, and (c) interaction of solid particles with the liquid (flowing in upward direction). Maude and Whitmore (1958) proposed the following empirical correlation for hindered settling velocity:

$$
V_{t s}=V_{t}\left(1-X_{v}\right)^{n_{t}}
$$


where $V_{t}$ and $V_{t s}$ are settling velocity and hindered settling velocity of solid particles, respectively. $X_{v}$ is solid volume fraction and $n_{t}=2.33$ for $R e_{P}>1000, n_{t}=4.375 R e_{P}^{-0.0875}$ for $0.3<R e_{P} \leq$ 1000 and $n_{t}=4.64$ for $R e_{P} \leq 0.3$, where $R e_{P}$ is particle Reynolds number.

\subsubsection{Particle Size Distribution}

As particles of different sizes are distributed in solid suspension, Baldi et al. (1978) suggested a mean particle size $d_{P(\text { ave })}$ for effective characterization of the system:

$$
d_{P(\text { ave })}=\frac{\sum_{i=1}^{N} n_{i} d_{i}^{4}}{\sum_{i=1}^{n} n_{i} d_{i}^{3}}
$$

where $d_{P(\text { ave })}$ represents average particle diameter and $n_{i}$ represents number or mass fraction of solid particle with $d_{i}$ size. $n_{i}$ can be calculated as:

$$
n_{i}=\frac{\text { mass of partciles in } i^{\text {th }} \text { size class }}{\text { mass of particle with diameter } d_{i}}
$$

Sphericity $(\psi)$ is generally used in quantification of particle shape in solid-liquid mixing. $\psi$ is given by:

$$
\Psi=\frac{\text { surface area of a non-spherical solid particle of the same volume }}{\text { surface area of a spherical solid particle }}
$$

\subsubsection{Effect of Physical Properties of Solid}

Size as well as density of solid particles have significant effect on suspension. Baldi et al. (1978) concluded that the effect of average particle size on just suspended speed $\left(N_{j s}\right)$ is $N_{j s} \propto d_{p}^{a}$, 
where $d_{p}$ is particle diameter and a is between 0.14-0.16. However, they commented that particles $d_{p}<200 \mu \mathrm{m}$ do not follow their model. Zwietering (1958) proposed $N_{j s} \propto d_{p}^{0.2}$ as correlation. Shamlou and Zolfagharian (1987) concluded the correlation as $N_{j s} \propto d_{p}^{0.17}$. Molerus and Latgel (1987) proposed correlation for fine particles $(\mathrm{Ar} \leq 40)$ as $N_{j s} \propto d_{p}^{0.56}$ whereas for coarse

particles (Ar $>40), N_{j s} \propto d_{p}^{0.14}$. Molerus and Latgel (1987) reported that exponents of densities for coarse and fine grained particles are different while estimating just suspended speed. Subbarao and Taneja (1979) proposed a model in which they indicated a negative exponent on $d_{p}$ in all conditions .Ditl and Rieger (1985) also proposed negative exponent of $d_{p}$. Their suggestions do not match with other researchers findings. Mak (1992) and Drewer et al. (1994) investigated the particle size effect and concluded that power number essentially increases with increase in average particle size. Tamburini et al. (2014) Concluded $N_{j s}$ to be independent of $d_{p}$ practically at all particle loadings. Particle shape and specifically, its orientations to the fluid flow affect the settling velocity, thus have impact on suspension (Paul et al., 2004).

\subsubsection{Effect of Physical Properties of Liquid}

Physical characteristics of liquid, specially density and viscosity of liquid have significant impact on solid-liquid suspension. According to Zwietering's equation, higher the values of liquid viscosity means higher the values of $N_{j s .}$. Yoshida et al (2012) concluded no dependence of $N_{j s}$ on kinematic viscosity for unsteadily stirred system. Pavlushenko et al. (1957) found $N_{j s}$ value proportional to $v^{-0.2}$ where $v$ is kinematic viscosity in unbaffled tanks. An increased fluid viscosity damps turbulent fluctuations and increases the drag coefficient. That is why, dependence of $N_{j s}$ on viscosity for different suspension mechanisms significantly differ. Zwietering (1958) concluded that a liquid with higher viscosity may show more initial difficulties in suspending the solid particles. Others argued that $N_{j s}$ should increase with decrease in kinematic viscosity since higher the viscosity, lower the particle terminal velocity. Tamburini et al. (2014) found that $N_{j s} \propto v^{0.13}$. Einenkel (1980) found the correlation as $N_{j s} \propto v^{-0.16}$. Mersmann et al (1998) proposed that just attained complete suspension may be controlled by either 'off-bottom lifting' or 'avoidance of settling' phenomenon. The effect of kinematic viscosity on these two phenomena is different. So, dependency of $N_{j s}$ on $v$ may be obtained as a result of the controlling phenomena. 


\subsubsection{Effect of Process Operating Conditions}

The process operating conditions such as solid concentration and liquid depth have effect on solid-liquid suspension. Zwietering showed the dependence of solid concentration on $N_{j s}$ by a correlation $N_{j s} \propto X^{0.13}$, where $X$ is mass ratio. Yoshida et al (2012) found that $N_{j s} \propto X^{0.10}$. Wu et al. (2002) suggested that particle loading should be taken into account for efficient and effective design and operation of the mixing tank. Bujalski et al. (1999) studied the impact of solid loadings on solid particles suspension. They observed increment in power number with increase in solids loading. Raghava Rao et al. (1988) found that mixing time depends on solid loading. They concluded that increase in solid loading increases the mixing time. Bubbico (1997) reported an increase of power number in solids suspension (estimated by taking weighted density) with solid loadings for all the different impellers used. Drewer et al (1994) presented power number in suspension as a function of solid concentration and types of impeller tested. Angst and Kraume (2006) investigated the effect of solids loading on power number $\left(N_{p}\right)$. Hicks et al. (1996) found that solids loading do affect the clouding height. Bittorf and Kresta (2003) found that a clear interface (cloud height) is formed for solid concentration higher than $10 \%$ (w/w) in a stirred vessel.

\subsubsection{Effect of Geometric Parameters}

Geometric configurations such as baffled/unbaffled, impeller types, impeller clearance, tank diameter and impeller diameter have impact on the degree of solid suspension. A brief survey of effect of impeller diameter is presented in Table (2.5-1). Machado et al. (2013) proposed that in case of a baffled mixing tank, a small diameter impeller with close impeller clearance could be very useful to minimize power consumption at $N_{j s}$. Myers et al. (2011) compared the mixing performance of some down-pumping stirrers of different types and diameter and found the average power reduction of $76 \%$ by using angle mounting unbaffled configuration, against standard vertical mounting baffled configuration. Jafari et al. (2012) studied the effect of stirrer type, clearance and stirrer speed on solid-liquid suspensions. By increasing off-bottom clearance, capability of a stirrer to suspend particles decreases due to reduction in energy dissipation and change in flow pattern (Kresta and Wood, 1993; Montante et al., 1999). Raghava Rao et al. (1988)

studied the influence of impeller design, impeller diameter and impeller speed on agitated solid- 
liquid suspensions and concluded that at critical impeller speed, these parameters practically do not influence the mixing time. Hicks et al. (1996) observed that stirrer to tank diameter ratio and stirrer clearance to vessel diameter ratio affect the cloud height significantly although solid loadings were kept constant at $10 \%$ for different runs.

\subsubsection{Effect of Agitation Conditions}

Conditions for agitation such as stirrer speed, stirrer power, liquid flow pattern and level of intensity (turbulence) in the tank affect the solid-liquid suspensions. Bittorf and Kresta (2003) reported that cloud height increases monotonically with increase in impeller speed. Rewatkar et al. (1991) and Michelletti et al. (2003) observed increment in power number $\left(N_{p}\right)$ with increase in stirrer speed. Angst and Kraume (2006) investigated the impact of stirrer speed on power number for slurry and concluded that power number for slurry increases with increase in stirrer speed. Rao et al. (1988) studied and analysed the effect of stirrer speed on mixing time and reported that power number increases with rise in stirrer speed. However, most of the researchers concluded that power number rises with rise in stirrer speed, disagreements in conclusion could be seen regarding effect of stirrer speed on power number. Pinelli et al. (2001) reported that power number declines with rise in stirrer speed. Most of the researchers did not consider radial concentration profiles in their works. However, Michelletti et al. (2003) obtained and analysed data at various radial positions and suggested that the effect of radial solid concentration profile should be taken into considerations while studying the distribution of solid particles in suspension. Kraume (1992) investigated and analysed the effect of stirrer speed and the level of suspension achieved on mixing times. He reported that the mixing time for two-phase (solid-liquid) suspensions, in certain cases, could be 10 times more than that in single phase mixing due to presence of solid particles. 
Table (2.5-1). Effect of impeller diameter -a brief survey

\begin{tabular}{|c|c|c|c|c|c|c|}
\hline Reference & Impeller & $\begin{array}{c}\text { Tank Size } \\
(\mathbf{m m})\end{array}$ & $\begin{array}{l}\text { Tank } \\
\text { Base }\end{array}$ & Clearance & $\mathrm{D} / \mathrm{T}$ & Results \\
\hline $\begin{array}{l}\text { Bujalski } \\
\text { (1986) }\end{array}$ & PBT6 & 290 & Flat & $\mathrm{C} / \mathrm{T}=0.25$ & $0.33-0.52$ & $N_{j s} \propto D^{-1.9}$ \\
\hline $\begin{array}{l}\text { Koutsakos } \\
\text { (1989) }\end{array}$ & PBT4 & $225-445$ & Profiled & $0.22 \leq \mathrm{C} / \mathrm{T} \leq 0.69$ & $0.23-0.57$ & $N_{j s} \propto D^{-106}$ \\
\hline $\begin{array}{l}\text { Susanto } \\
\text { (1989) }\end{array}$ & Propeller & 188 & Flat & $\mathrm{C}=$ constant & $0.34-0.41$ & $N_{j s} \propto D^{-1.69}$ \\
\hline $\begin{array}{l}\text { DeRitter } \\
(1990)\end{array}$ & PBT6 & 292 & Flat & $0.5 \leq \mathrm{C} / \mathrm{D} \leq 1$ & $0.22-0.35$ & $\begin{array}{c}N_{j s} \propto D^{-1.74,} \\
C / D=0.5 \\
N_{j s} \propto D^{-1.68,} \\
C / D=1\end{array}$ \\
\hline $\begin{array}{l}\text { Zolfagharian } \\
\text { (1990) }\end{array}$ & $\begin{array}{l}\text { Propeller } \\
\text { PBT4 }\end{array}$ & $240-297$ & Flat & $\mathrm{C} / \mathrm{T}=0.25$ & $0.20-0.63$ & $N_{j s} \propto D^{-1.66}$ \\
\hline $\begin{array}{l}\text { Rao et al. } \\
\text { (1988) }\end{array}$ & PBT6 & $570-1000$ & Flat & $0.17 \leq \mathrm{C} / \mathrm{T} \leq 0.5$ & $0.16-0.66$ & $N_{j s} \propto D^{-1.16}$ \\
\hline $\begin{array}{l}\text { Chapman } \\
\text { (1981) }\end{array}$ & PBT4 & 560 & Flat & $\mathrm{C} / \mathrm{T}=0.25$ & $0.25-0.50$ & $N_{j s} \propto D^{-1.5}$ \\
\hline $\begin{array}{l}\text { Zwietering } \\
\text { (1958) }\end{array}$ & Propeller & $154-600$ & Flat & $\mathrm{C} / \mathrm{T}=0.25,0.4$ & $0.16-0.63$ & $N_{j s} \propto D^{-1.67}$ \\
\hline
\end{tabular}

where PBT $=$ Pitch blade turbine, $C=$ Clearance, $D=$ Diameter of impeller, $T=$ Tank diameter, and $N_{j s}=$ Just Suspension speed 


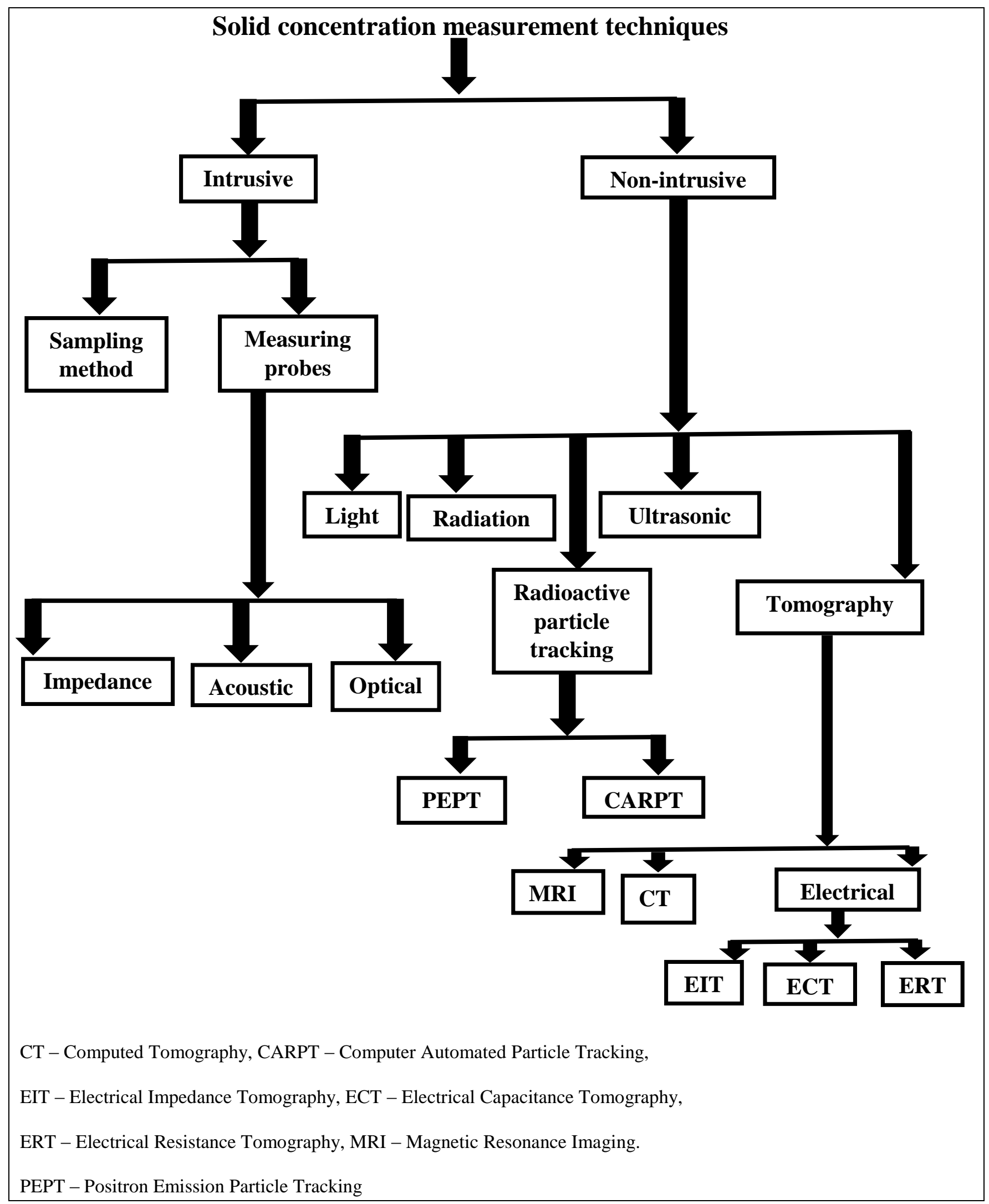

Figure (2.6-1). Classification of solid concentration measurement techniques. 


\subsection{Solid Concentration Measurement Techniques}

Different techniques employed to measure solid particle concentration in an agitated tank is shown in Figure (2.6-1). These techniques are broadly classified into two categories: (a) invasive (intrusive) and (b) non-invasive (non-intrusive).

\subsubsection{Invasive Measurement Techniques}

Invasive particle concentration measurement techniques are classified into two categories: (a) sampling method and (b) measuring probes.

\subsubsection{Sampling Method}

The sampling method was the oldest technique employed to determine solid particle distribution in agitated vessels. Due to simplistic approach to withdraw samples from solid-liquid suspensions at different locations of the agitated vessels and measure solid concentration of the sample, this method was employed to access the distribution of solid particles (White and Sumerford, 1933; Barresi and Baldi, 1987; Buurman et al., 1985). The sample is withdrawn with the help of a measuring probe and sampling tube to perform such operation. Samples that could actually represent the local solid concentration are very difficult to withdraw from an agitated vessel (Nienow, 1985; Mactaggart et al., 1993). Rushton (1965) and Sharma and Das (1980) proposed empirical equations to explain the particle concentrations obtained by the sampling method.

The drawback of this method is its high intrusive nature. The other shortcoming is that the solid concentration in the sample may be different from that taken at the sampling location of the tank due to error in withdrawing the sample itself. Research works (Godfrey and Zhu, 1994; Nasrel-din et al., 1996) reported that this method does not provide results, significant enough because of the influence of shape of the probe and location of the mixing vessel.

Sampling method was also used for floating particles. Floating solid suspension in a stirred vessel was investigated by different researchers (Kuzmanic and Kessler, 1997; Kondo et al., 2008). Results obtained from their investigation showed that the probability of getting sample that could represent the floating suspended solids from an agitated vessel at complete suspension state using sampling method is almost nil. They came to conclusion that although sampling errors cannot be 
totally reduced, they can be reduced to a certain extent by altering suspension conditions. This method should be applied in practice only when other measuring techniques are not available and feasible. To obtain reliable data from this method, extreme care and caution is required.

\subsubsection{Measuring Probes}

This technique can be classified into three sub-groups: (a) impedance (b) optical, and (c) acoustic. This division is based on the variables they measure.

\subsection{Impedance Techniques}

Impedance methods make use of two or four electrodes to measure impedance (complex ratio of the voltage to the current in an a.c. circuit). Solid concentrations between these electrodes influence impedance values thus measured. These techniques were employed to determine particle distribution in two-phase systems (McKee et al., 1995; Paglianti and Pintus, 2001; Brunazzi et al., 2001). Hewitt (1978) and Wang et al. (2000) also used impedance techniques to measure the particle concentration and distribution in solid-liquid mixing.

These techniques can be employed for local concentration measurements (Takenaka and Takahashi, 1996; Andreussi et al., 1999) as well as for integral measurements (Andreussi et al., 1988; Brunazzi et al., 2001). These methods are recommended for high particle concentration. They are highly influenced by local particle concentration, flow pattern and operating conditions. Micheletti et al. (2003) used a Y-shaped body (made of Perspex) and a handle (made of stainless steel) as a conductivity probe to investigate impeller off-bottom clearance, particle diameter and concentration effects in a standard geometry stirred vessel. Shirhatti et al. (2006) used a four electrodes conductivity method to access the minimum agitation speed at complete solid suspension. Spidla et al. (2005) used this technique to obtain concentration profiles for moderately concentrated suspension. The authors found that a higher degree of suspension homogeneity is directly related to the use of smaller particles at higher average concentrations.

Brunazzi et al. (2004) used an innovative probe to assess solid-hold up at different heights in agitated tank reactor with high solid concentration using electrical impedance technique. The advantage of this probe is its non-intrusive nature since it uses two ring electrodes located on the tank wall and shaft respectively. These can be placed at different elevations along the axial 
direction to get measurements. The probe can be used for lab scale as well as for full scale stirred tanks. The drawback of this technique is in difficulty of identifying the measuring volume of the suspensions. The data obtained from this technique is considered to be averaged in radial and somewhat in axial directions.

\subsection{Optical Techniques}

Optical techniques make use of attenuation of light beam from the source to the receiver. Lu et al. (1993) used photoelectric capillaries to investigate local solid concentration. Shan et al. (2008) used fibre optic probes instead of photoelectric capillaries for measurement of local solid concentration. (Angst and Kraume, 2006; Alban et al., 2004) used endoscopes to assess the local particle concentrations.

Shan et al. (2008) used a fibre-optic probe consisting of one bundle of quartz fibre working as light projector and other bundle of quartz fibre working as light receiver to investigate solid-liquid unbaffled vessels in order to measure solid particle concentration.

The endoscope system employed by Angst and Kraume (2006) is based on back scattering principle. The mean value of light intensity received from back scattering is evaluated. The value thus obtained directly corresponds to the solid concentration at the point of measurement. The intrusive disturbance can be avoided in this technique.

Sessiecq et al. (1999) used a turbidity sensor to get axial profiles of local solid particle concentration. Optical fibres pass a polychromatic light beam to the sensor. The particles in suspension then scatter the beam crossed through the sensor window. Optical fibres are again used to lead it to a photodiode array spectrophotometer to obtain the suspension turbidity. The measured turbidity value thus obtained can be used to determine particle concentration for soli-liquid suspension.

\subsection{Acoustic Techniques}

In acoustic methods, sound speed and amplitude of a transmitted sound beam is measured in a continuous liquid phase. When system has more than one phase, then the properties become different. When a liquid contains gas bubbles or solid particles, the sound wave is transmitted and reflected at the boundary of two phases with varying impedances. Bamberger et 
al. (1998) employed acoustic techniques to measure the local solid concentration. Zheng and Zhang (2004) reported that the sound energy loses faster in an inhomogeneous system compared to that of a homogeneous one. They observed remarkable changes in velocity and attenuation of sound due to absorption and scattering in a homogeneous medium. Corresponding dispersed phase hold-up can be determined based on this attenuation (Chang et al., 1984).

Alba et al. (1999) developed a device that can measure concentration and solid distribution of samples of low contrast density and high contrast density. This technique uses passing ultrasonic waves of different frequencies through the media and assessing the attenuation at all frequency values. Solid concentration and distribution thus can be determined based on spectrum attenuation.

Bamberger and Greenwood (2004) employed a single frequency ultrasonic wave measurement method to measure the slurry ( silicon dioxide- water) concentration in an open vessel which is helpful in monitoring the progress of the mixing. The ultrasonic sensor uses a transmitter and a receiver, placed parallel and coaxial and they are allowed to suspend in the system. The transmitter sends a frequency pulse ranging from 0.1 to $3 \mathrm{MHz}$ through the system to the receiver. Signals thus received is detected by a peak detector.

All the intrusive techniques have the problem of having the presence of probe inside the agitated tank which may affect flow pattern of the local field. Thus, the presence of probe may alter the results. Most of the times, solid distribution throughout the media is required but these techniques only give point wise assessments. Intrusive techniques should be applied only when non-intrusive techniques become ineffective due to various reasons such as presence of dense solid suspensions in light attenuation techniques.

\subsubsection{Non-invasive Techniques}

The major non-invasive techniques can be classified into five sub-groups: (a) light attenuation techniques (b) radiation methods (c) ultrasonic/ acoustic attenuation methods (d) radioactive particle tracking techniques, and (d) tomographic methods. 


\subsubsection{Light Techniques}

Light attenuation method is the most commonly used light techniques. It consists of a diode which emits light as source and a photodiode (made of silicon) which receives the signal as a receiver. The attenuation in a light beam is measured while passing through the mixing tank. Degree of attenuation increases when the light goes through solid suspensions compared to that through pure liquid. Bohnet and Niesmak (1980) and Fajner et al. (1985) employed light attenuation methods to measure particle concentration. These techniques provide accurate results as they give solid concentration averaged over the line of the light beam. Angst and Kraume (2006) adopted an approximation method to represent average solid concentration in an entire horizontal section by making use of the average solid concentration thus measured along the line of a light beam. Barresi and Baldi (1987) assumed the radial profiles to be flat. In reality, both azimuthal and radial profiles cannot be neglected (Tamburini et al., 2009; Tamburini et al., 2011) particularly at bottom of the mixing tank (Guida et al., 2009; Guida et al., 2010). Micheletti and Yianneskis (2004) made use of Laser Doppler Anemometry (LDA) technique to assess the continuous phase velocity. They first matched the refractive index of both phases.

Unadkat et al. (2009) suggested an advanced method using a combination of fluorescent particles and particle image velocimetry (PIV) technique to assess particle suspensions in stirred tanks. They measured turbulence characteristics such as root mean square (rms) velocities and dissipation rate. They used two different cameras to observe the flow pattern in both solid and liquid media simultaneously. Authors proposed a specific method to assess solid distribution in a vertical diametrical section of the tank based on image analysis. The resulting concentration maps thus obtained take the relative distribution of solids in captured in both cameras according to the authors.

Tamburini et al. (2009) employed laser sheet and image analysis (LSIA) method to determine solid distribution in dilute suspensions. It is also a non-invasive method. The drawback of this technique is that it can be only used for dilute solid-liquid suspensions as laser ray does not pass through dense suspensions.

Houcine et al. (1996) and Guillard et al. (2000) used planar laser induced fluorescence (PLIF) method to assess particle concentration in a continuously stirred flow field. Doh et al. 
(2013) employed very simple visualisation system (LCD monitor and a camcorder) to measure local particle concentration in the solid-liquid system.

Ferreira et al. (1994) measured solids concentration profiles using a light scattering technique associated with the refractive index matching procedure. They used this non-intrusive technique and compared results with other intrusive techniques. This technique is easy to apply and requires less sophisticated equipment.

These light based techniques have limitations based on concentration of suspensions. These techniques cannot be used for dense solid suspensions as light is not possible to pass through these systems. This shortcoming can be overcome by checking the refractive indices of both phases.

\subsubsection{Radiation Techniques}

Radiation techniques use $X$-rays or neutron rays to measure particle concentration (Fournier et al., 1993; Hewitt, 1978). When these rays pass through solid-liquid suspensions, attenuated radiations are received. The principle of this method is almost identical to that of light attenuation technique (Boyer et al., 2002). Mondy et al. (1986) and Milliken et al. (1989) employed these techniques to evaluate phase hold-up. This technique should not be used in industrial stirred tanks due to radiation hazards and high cost (Powell, 2008). Hewitt (1978) suggested that these techniques can be used in an invasive manner. While using radiation techniques in an invasive way, a great care should be given in selection of proper probes.

\subsubsection{Ultrasonic Attenuation Techniques}

Ultrasonic attenuation methods are similar in principle to the corresponding invasive methods. The major difference is in the location of the measuring sensors. The sensors are fixed outside the system to avoid intrusiveness. An ultrasonic wave can penetrate through extremely dense suspension which is practically not possible for the light. Uchida et al. (1989) and Vatanakul et al. (2005) used ultrasonic attenuation methods to study fluidized beds. Zheng and Zhang employed ultrasonic attenuation method to investigate particle suspension and distribution in fluidized beds. 
Ren et al. (2008) proposed non-intrusive acoustic emission method based on multi-scale analysis to determine slurry suspension height and critical agitation speed for complete suspension. Authors compared the results with visual observation of the just suspended speed to validate their method.

\subsubsection{Radioactive Particles Tracking Methods}

Radioactive particles tracking methods can be classified into two categories: (a) Positron Emission Particles Tracking (PEPT) technique and (b) Computer Automated Particles Tracking (CARPT) technique.

PEPT and CARPT are non-invasive techniques which are capable of solving Lagrangian particles trajectory in dense and opaque media using opaque devices. They are very useful in flow visualization to study complex fluid motion in stirred tanks.

\subsection{PEPT Technique}

PEPT consists of three components. They are: (a) tracer particle, (b) detector (positron camera, and (c) tracking algorithm (Fangary et al., 2000; Guida et al., 2009). Positron emitting isotope is used to label the tracer particle. A location tracking algorithm is used to compute location of the tracer. Fangary et al. (2000) employed this technique to assess the distribution of solid particles in solid-liquid system. The particle tracer emits a positron which eventually destroys a pair of $\gamma$-rays (emitted by an electron) coming from other directions. The detectors located outside the agitated tank can detect these pair of $\gamma$-quanta. Only those coincidence events are recorded where $\gamma$-quanta are tracked by both the detectors simultaneously. Parker et al. (1993) used location tracking algorithm to calculate the Lagrangian radioactive tracer trajectory. The detectors cannot detect all of $\gamma$-rays because some of them may scatter before detection. The location tracking algorithm employed can discard these events (Barigou, 2004).

For solid-liquid phase in stirred vessels, two different tracer particles are used to simultaneously track both phases (Guida et al., 2009). Wittmer et al. (1998) suggested to assume ergodicity when the possibility of tracer reaching throughout the system is substantially high. Ergodicity is a theoretical consideration that presumes a labelled tracer can represent either solid (dispersed) or liquid (continuous) phase. 


\subsection{CARPT Technique}

CARPT techniques adopt the basic principle almost similar to PEPT techniques. (Dudukovic, 2002; Guha et al., 2007) employed these techniques to assess the distribution of particles in two phases system. CARPT system comprises of sixteen scintillating detectors fixed on supports (made of aluminium) and arranged on an orthogonal base. The configuration is adopted such that the particle can be visible by each of sixteen detectors in the vessel (Rammohan et al., 2001). An active crystal (made of sodium iodide) is placed in each detector. Rammohan et al. (2003) shed light on advantages and drawbacks of PEPT/CARPT methods. Authors found three kinds of probable errors in PEPT/CARPT assessment. However these errors can be minimized (Parker et al., 1993; Larachi et al., 1997; Rammohan et al., 2001; Guida et al., 2009).In CARPT, significantly large $2300 \mu \mathrm{m}$ tracer is used. But in PEPT, a $600 \mu \mathrm{m}$ tracer is employed. Barigou (2004) showed that a $600 \mu \mathrm{m}$ tracer follows the fluid streamlines. Other aspects of PEPT technique were explained by Guida et al. (2009). The obvious shortcomings of radioactive particle tracking techniques are high cost equipment and radiation hazards (Boyer et al., 2002). These techniques should be only used for complete suspension conditions.

\subsubsection{Tomographic Techniques}

Tomographic methods were proposed recently for industrial applications (Williams and Beck, 1995). These techniques use phase volumetric fraction in measuring distribution. Measurements are done at different positions to provide average values for particle distribution. Reliable and relevant reconstruction algorithm is employed to analyze the acquired signals. Three major tomographic techniques that are used to investigate multiphase flow are: (a) Magnetic Resonance imaging (MRI) technique (b) Computed Tomographic (CT) technique, and (c) Electrical Tomographic (ET) technique.

\subsection{MRI Technique}

MRI is non-intrusive method employed to investigate multiphase flows (Majors et al., 1989; Sinton and Chow, 1991; Altobelli et al., 1991; Stapf and Han, 2006; Powell, 2008). In this method, a magnetic field is applied to a system to receive sample responses of different intensities in the frequency domain. When this technique is used for solid-liquid suspension, the signal (magnitude) received at each frequency provides the local volume fraction of the continuous phase. 
It is then easier to obtain local solid volume fraction. First a reference image is acquired for liquid only. Later MRI is employed to obtain images of a sample for both solid and liquid phases. By comparing the images thus obtained for both conditions, the results can be analyzed and interpreted. MRI can be used to measure distribution of droplets size. This technique can be used for very high solid suspensions without matching the refractive indices. MRI apparatus is very expensive and needs very skilled person to operate.

\subsection{Computed Tomography (CT)}

Computed tomography (CT) used by (Kumar et al., 1995; Chaouki et al., 1997; Schmitz and Mewes, 2000; Dudukovic, 2002) has very simple principle. By measuring attenuation of photon rays through two phase mixtures, it is easier to predict distribution of both phases in the scanned cross-section as different phases show different attenuations. The difference of densities in both phases provide the basis for attenuation measurements. The shortcomings of this technique are related to cost, safety and high power requirements for high solid loading system. This method should be used for stationary flow (Boyer et al., 2002).

\subsection{Electrical Tomography (ET)}

Electrical tomography makes use of dielectric properties of media for imaging. They are further classified into three sub-groups: (a) Electrical Resistance Tomography (ERT), (b) Electrical Capacitance Tomography (ECT), and (c) Electrical Impedance Tomography (EIT). In ECT, capacitance is measured (Warsito and Fan, 2001) and in ERT, conductance is measured. In case of EIT, electrical impedance is measured for analysis. The sensing electrodes are fixed outside

the mixing tank in ECT but they are fixed inside the system in ERT. A reliable and proper reconstruction algorithm interprets the measured values to solid concentration.

In ERT, multiple electrodes are used to make reconstruction easier. Conductivity distribution inside a vessel is measured at adjacent, opposite, diagonal and conducting boundary protocols (Hosseini et al., 2010a). Concentration gradients as a function of spatial gradients of electrical conductivity thus can be measured. 
In ECT, electrical capacitance is measured between pairs of electrodes to reconstruct the image of medium present. Capacitance values can be measured by exciting electrodes one by one (Warsito and Fan, 2001).

Electrical tomographic technique is received well in industrial processes due to simplistic nature and high speed imaging capacity (Dyakowsky et al., 2000). This technique is very easy to use with lower costs. It may be difficult to obtain accurate results (Mann et al., 1997) if noise present in the system is not reduced completely. Sometimes, Complex reconstruction algorithms may be required to obtain better results. Stephenson et al. (2008) addressed various factors which can affect the images obtained in EIT.

Electrical tomography systems are widely used in stirred tanks (McKee et al., 1995; Williams et al., 1996; Mann et al., 1997; Stanley et al. 2002; Bolton and Primrose, 2005; Rodgers et al., 2009). Williams et al. (1996) explained the applications of ERT in measuring concentration of solid particles in a slurry reactor taking particle diameter, impeller type and impeller speed as key variables. Bolton and Primrose (2005) described the use of electrical tomography in industrial processes. They investigated concentration of solids in stirred tanks and crystallizers. Rodgers et al. (2009) showed the capability of EIT to monitor reactive stirred systems. Harrison et al. (2012) investigated solids suspension using ERT. Jafari et al. (2012) validated the use of ERT for particle measurement. Review on ERT major applications to chemical engineering has been reported by Sharifi and Young (2013). A brief literature survey of ERT is presented in Table (2.6-1). 
Table (2.6-1). A brief literature survey of ERT in solid-liquid mixing

\begin{tabular}{|c|c|c|c|}
\hline References & $\begin{array}{l}\text { Tomography Method/System } \\
\text { under investigation }\end{array}$ & Objective & Findings/Comments \\
\hline McKee et al.,1995 & ERT,PET/Solid-liquid in tank & $\begin{array}{l}\text { To determine } N_{j s} \text { and effect of } \\
\text { impeller speed on concentration } \\
\text { profile }\end{array}$ & $\begin{array}{l}\text { Good agreement with Zweitering's } \\
\text { correlation }\end{array}$ \\
\hline Williams et al., 1996 & ERT/Solid-liquid in vessel & $\begin{array}{l}\text { To study and analyse the effect of } \\
\text { stirrer type and particle size }\end{array}$ & $\begin{array}{l}\text { Concentration profile determined } \\
\text { with four planes to some extent but } \\
\text { needed more data }\end{array}$ \\
\hline Mann et al., 1997 & $\begin{array}{l}\text { ERT/Gas-liquid, solid-liquid in } \\
\text { vessel }\end{array}$ & $\begin{array}{l}\text { To develop a model to predict the } \\
\text { conductivity in three dimensional }\end{array}$ & $\begin{array}{l}\text { Successful in calculating the } \\
\text { conductivity spatially/Useful for } \\
\text { post processing }\end{array}$ \\
\hline Williams et al., 1998 & $\begin{array}{l}\text { ERT,ECT/Gas-liquid, solid- } \\
\text { liquid in vessel }\end{array}$ & $\begin{array}{l}\text { To test the ability of tomography } \\
\text { system for monitoring the mixing } \\
\text { behaviour online }\end{array}$ & $\begin{array}{l}\text { Successful monitoring in both } \\
\text { aqueous and organic media }\end{array}$ \\
\hline West et al., 1999 & $\begin{array}{l}\text { ERT/PET/Solid-liquid in } \\
\text { vessel }\end{array}$ & $\begin{array}{l}\text { To test ERT and PET in } \\
\text { monitoring the system }\end{array}$ & $\begin{array}{l}\text { PET was unable to pick the changes } \\
\text { within the system due to low } \\
\text { temporal resolution }\end{array}$ \\
\hline Stanley et al., 2002 & ERT/Solid-liquid in vessel & To control the precipitation rate & Mixing was far from ideal \\
\hline Wang et al., 2003 & $\begin{array}{l}\text { ERT/Solid-liquid, swirl flow in } \\
\text { pipe }\end{array}$ & $\begin{array}{l}\text { To quantify the solid } \\
\text { concentration in pipe }\end{array}$ & $\begin{array}{l}\text { A critical velocity for the flow was } \\
\text { determined above which there is a } \\
\text { drastic concentration gradient }\end{array}$ \\
\hline Madupu et al., 2005 & ERT/Solid-liquid & $\begin{array}{l}\text { To measure the solid level in an } \\
\text { underground storage tank }\end{array}$ & $\begin{array}{l}\text { Solid height was measured, the } \\
\text { solid-liquid interface was clearly } \\
\text { detected }\end{array}$ \\
\hline
\end{tabular}


Table (2.6-1). Continued (1)

\begin{tabular}{|c|c|c|c|}
\hline References & $\begin{array}{l}\text { Tomography Method/System } \\
\text { under investigation }\end{array}$ & Objective & Findings/Comments \\
\hline Ricard et al., 2005 & $\begin{array}{l}\text { ERT/Liquid-liquid, solid- } \\
\text { liquid in tank }\end{array}$ & $\begin{array}{l}\text { To mimic mixing process in } \\
\text { pharmaceutical production } \\
\text { reactor }\end{array}$ & $\begin{array}{l}\text { Major achievement in geometry } \\
\text { optimization }\end{array}$ \\
\hline Stanley, 2006 & ERT/Solid-liquid in vessel & $\begin{array}{l}\text { To evaluate the state of } \\
\text { precipitation by measuring the } \\
\text { solid concentration }\end{array}$ & $\begin{array}{l}\text { Kinetic of precipitation was studied, } \\
\text { solid concentration profile and images } \\
\text { were made }\end{array}$ \\
\hline Giguere et al., 2008 & ERT/Solid-liquid in pipe & $\begin{array}{l}\text { To study the effect of solid } \\
\text { concentration profile }\end{array}$ & $\begin{array}{l}\text { Fair noise reduction through different } \\
\text { conductivity calculation algorithm to } \\
\text { result better concentration contour }\end{array}$ \\
\hline Hosseini et al., 2010a & ERT/Solid-liquid in tank & $\begin{array}{l}\text { To quantify the degree of } \\
\text { homogeneity by using } \\
\text { concentration profiles }\end{array}$ & $\begin{array}{l}\text { Successful in determining the quality } \\
\text { of solid-liquid mixing in an agitated } \\
\text { vessel/ A new approach to determine } \\
\text { homogeneity was proposed }\end{array}$ \\
\hline Tahvildarian et al., 2011 & ERT/Solid-liquid in vessel & $\begin{array}{l}\text { To assess the level of } \\
\text { homogeneity for micron-sized } \\
\text { particles in slurry }\end{array}$ & $\begin{array}{l}\text { Successful in determining the local } \\
\text { mixing quality in a slurry reactor }\end{array}$ \\
\hline Harrison et al., 2012 & ERT/Solid-liquid in tank & $\begin{array}{l}\text { To assess solid concentration } \\
\text { homogeneity }\end{array}$ & $\begin{array}{l}\text { A new mixing index was proposed to } \\
\text { quantify homogeneity in both axial } \\
\text { and radial directions }\end{array}$ \\
\hline Carletti et al., 2014 & ERT/Solid-liquid in vessel & $\begin{array}{l}\text { To analyse solid } \\
\text { concentration and distribution } \\
\text { in dense solid-liquid system }\end{array}$ & $\begin{array}{l}\text { Successful in analysing solid } \\
\text { concentration distribution/A new } \\
\text { mixing index for assessment of local } \\
\text { mixing quality was proposed }\end{array}$ \\
\hline
\end{tabular}




\subsection{Forces Acting on Solid Particles}

Forces acting on solid particles moving through a liquid medium are of great importance. Force $(F)$ acting on a solid particle can be written using Newton's law of motion as:

$$
F=m a=\rho_{P} v_{P} \frac{d V}{d t}
$$

where $m, a, \rho_{P}, v_{P}, V$, and $t$ are mass of particle, acceleration of particle, particle density, volume of particle, velocity and time respectively. Force can be expressed in energy gradients as:

$$
F=-\frac{d U}{d x}
$$

where term in right hand side represents energy gradient. Different types of forces acting on particles are discussed in details.

\subsubsection{Gravitational Force}

Gravitational field strength can be expressed in vectorial form as:

$$
\boldsymbol{g}=\frac{\boldsymbol{F}_{\boldsymbol{G}}}{m}
$$

where $\boldsymbol{F}_{\boldsymbol{G}}, \boldsymbol{g}$, and $\mathrm{m}$ are gravitational force, acceleration due to gravity $\left(9.81 \mathrm{~m} / \mathrm{s}^{2}\right.$ at surface of the earth) and mass, respectively. Motion of very small particles due to gravity will be slow due to low terminal velocity of these particles. To increase motion of these small particles, centrifugal force can be applied and can be expressed as: 


$$
F_{c}=m r \omega^{2}=\rho_{P} v_{P} r \omega^{2}
$$

where $F_{c}, \omega, v_{P}, \rho_{P}, m$, and $r$ are centrifugal force, angular velocity, volume of particle, density of particle, mass of particle and distance between particle and rotation axis, respectively. The particles tend to move in radial outward directions under the influence of centrifugal force. Gravitational force for unit mass can be defined as:

$$
\boldsymbol{F}_{G}=\frac{\left(\rho_{P}-\rho_{C}\right)}{\rho_{P}} \boldsymbol{g}
$$

where $\boldsymbol{F}_{G}, \boldsymbol{g}, \rho_{P}$, and $\rho_{c}$ are gravitational force, gravity, particle density and continuous phase density, respectively.

\subsubsection{Buoyant Force}

Buoyancy is a force that can determine how well an object can float or how high it can rest in the fluid (Mott, 2005). According to Archimedes' principle, any object in a fluid experiences buoyancy equal to the weight of the volume of liquid, which is displaced. The concept of buoyancy is almost similar to that of density. Buoyant force $\left(\boldsymbol{F}_{B}\right)$ can be expressed as:

$$
\boldsymbol{F}_{B}=\rho_{c} v_{P} \boldsymbol{g}
$$

Buoyant force depends on density of liquid $\left(\rho_{c}\right)$, volume of the particle $\left(v_{P}\right)$ and gravity $(g)$. Both buoyant and gravitational forces act on a body whether it is moving or static in the liquid. Buoyant force under the influence of a centrifugal field can be defined as: 


$$
\boldsymbol{F}_{c b}=\rho_{c} v_{P} r \omega^{2}
$$

where $\boldsymbol{F}_{c b}$ is buoyant force under a centrifugal field.

\subsubsection{Force due to Pressure Gradient}

In absence of particles, the surrounding fluid will occupy the particles space. The force needed to move the fluid which will occupy the particles space, if they are not present is referred as force due to pressure gradient (Crowe et al., 1998):

$$
\boldsymbol{F}_{P}=\frac{\rho_{c}}{\rho_{p}} \boldsymbol{u}_{P} \frac{\partial \boldsymbol{u}_{c}}{\partial X}
$$

where $\boldsymbol{F}_{P}, \boldsymbol{u}_{c}, \boldsymbol{u}_{P}$, are force due to pressure gradient for unit mass, continuous (liquid) phase velocity and particle velocity, respectively.

\subsubsection{Added (Virtual) Mass Force}

The added mass force is responsible for the acceleration of the fluid that surrounds the particles. The particle which will occupy the fluid space exert force on the fluid to displace it from its space (Crowe et al., 1998). The rate needed to alter the energy (kinetic) of the liquid surrounding a solid particle gives added mass force $\left(\boldsymbol{F}_{A}\right)$ and can be given as:

$$
\boldsymbol{F}_{A}=\frac{\rho_{c}}{2 \rho_{p}} \frac{d}{d t}\left(\boldsymbol{u}_{c}-\boldsymbol{u}_{P}\right)
$$




\subsubsection{Acoustic Force}

Acoustic force $\left(\boldsymbol{F}_{a c}\right)$ is encountered when colliding particles have dimensions equitable to the sound wavelength (Kapishnikov et al., 2006). For $\left(k_{a} R_{P}<<1\right), \boldsymbol{F}_{a c}$ can be expressed as:

$$
\boldsymbol{F}_{\boldsymbol{a c}}=\frac{2 \pi\left(k_{a} R_{P}\right)^{3}\left(2 \bar{E}_{s t}\right)}{k_{a}^{2}} \Phi\left(\frac{\rho_{p}}{\rho_{c}}, \frac{c_{P}}{c_{c}}\right) \sin \left(2 k_{a} \boldsymbol{r}_{\mathbf{0}}\right)
$$

and

$$
\Phi\left(\frac{\rho_{P}}{\rho_{C}}, \frac{c_{P}}{c_{C}}\right)=\frac{1}{3}\left[\frac{5\left(\frac{\rho_{p}}{\rho_{c}}\right)-2}{2\left(\frac{\rho_{p}}{\rho_{c}}\right)+1}-\frac{1}{\left(\frac{\rho_{p}}{\rho_{c}}\right)\left(\frac{c_{P}}{c_{C}}\right)^{2}}\right]
$$

where $k_{a}=2 \pi / \lambda . \bar{E}_{s t}, k_{a}, \lambda, R_{P}$, and $\boldsymbol{r}_{\mathbf{0}}$ are energy density of the developed waves (time averaged), wave number, wavelength, radius of the particle and vector normal to the force nodes, respectively. $\frac{c_{P}}{c_{c}}$ is the ratio of velocity of sound in the solid particle to that in the liquid medium.

\subsubsection{Shear Force}

Shear force is a dynamic force that exists when (a) there is relative velocity between the liquid medium and the particles and (b) there is no slip condition. All the forces mentioned above can exist even both the particles and the fluid are stagnant. But shear force does not exist if relative acceleration between the solid particle and the liquid is zero. This force is responsible for the deformation of a particle. Shear force $\left(F_{S}\right)$ can be given as:

$$
F_{S}=\tau_{x y} S_{A}
$$

where $\tau_{x y}$ and $S_{A}$ are shear stress and surface area of particle respectively. 


\subsubsection{Drag Force}

Drag force is a resistive force that acts on a body moving in a liquid medium in the direction parallel to the relative motion of the fluid (Mott, 2005). They are of two types: form drag (influenced by cross-sectional area) and surface drag (influenced by smoothness of surface). By assuming, no change in flow pattern and no particle-particle interaction, drag force $\left(\boldsymbol{F}_{D}\right)$ can be evaluated (Morsi and Alexander, 1972) :

$$
\boldsymbol{F}_{D}=\frac{18 \mu_{c}}{\rho_{p} d_{P}^{2}} \frac{C_{D} R e_{P}}{24}\left(\boldsymbol{u}_{c}-\boldsymbol{u}_{P}\right)
$$

where $C_{D}, R e_{P}, \mu_{c}$, and $d_{P}$ are drag coefficient, particle Reynolds number, viscosity of the fluid, and particle diameter, respectively. $C_{D}$ depends on particle velocity, particle shape, smoothness of particle surface and viscosity of the continuous (fluid) phase. $R e_{P}$ can be used in characterising $C_{D}$ as:

$$
R e_{P}=\frac{d_{P}\left|\boldsymbol{u}_{\boldsymbol{c}}-\boldsymbol{u}_{\boldsymbol{P}}\right|}{v_{c}}
$$

where $v_{c}$ is kinematic viscosity of the medium. Drag coefficient for a spherical particle has different values in following three regimes:

(a) Laminar regime or Stokes regime $\left(R e_{P}<1\right)$ : In this regime, viscous forces are dominant so that inertial effects can be neglected. For a spherical particle in this regime, Stokes developed the following correlation for the drag force $\left(\boldsymbol{F}_{D}\right)$ :

$$
\boldsymbol{F}_{D}=3 \pi \mu_{C} d_{P}\left(\boldsymbol{u}_{c}-\boldsymbol{u}_{P}\right)
$$


The drag coefficient in this regime for spherical particle can be written as:

$$
C_{D}=\frac{24}{R e_{P}}
$$

(b) Intermediate regime $\left(1<R e_{P}<1000\right)$ : In this regime, the inertial effects need to be taken into account while predicting the drag coefficient. Data obtained from experimental measurements should be used to develop a correlation for drag coefficient.

(c) Turbulent regime or Newton's regime $\left(R e_{P}>1000\right)$ : In this regime, the drag coefficient $\left(C_{D}\right)$ does not rely on Reynolds number or velocity and becomes approximately constant. For a spherical particle in this regime, $C_{D}$ becomes:

$$
C_{D} \cong 0.44
$$

When particle Reynolds number nearly approaches $2 \times 10^{5}, C_{D}$ abruptly decreases. A brief summary of drag correlations in laminar and turbulent regime is presented in Table $(2.7-1,2)$. 
Table (2.7-1). Drag coefficient correlations for spherical particles

\begin{tabular}{|c|c|c|}
\hline References & $R e_{P}$ range & Correlation \\
\hline \multirow[t]{4}{*}{ Clift et al. (1978) } & $0.01 \leq R e_{P} \leq 20$ & $C_{D}=\frac{24}{R e_{P}}\left[1+0.1315 R e_{P}^{0.82-0.05 w}\right]$ \\
\hline & $260 \leq R e_{P} \leq 1500$ & $\log C_{D}=1.6435-1.124 w+0.1558 w^{2}$ \\
\hline & $1500 \leq R e_{P} \leq 1.2 \times 10^{4}$ & $\log C_{D}=-2.4571+2.5558 w-0.9295 w^{2}+0.1049 w^{3}$ \\
\hline & $4.4 \times 10^{4} \leq R e_{P} \leq 3.38 \times 10^{5}$ & $\log C_{D}=-4.339+1.5809 w-0.1546 w^{2}$, where $w=\log R e_{P}$ \\
\hline Flemmer and Banks (1986) & $R e_{P}<8.6 \times 10^{4}$ & $C_{D}=\frac{24}{R e_{P}} 10^{E} ; E=0.261 R e_{P}^{0.369}-0.105 R e_{P}^{0.431}-\frac{0.124}{1+\left(\log R e_{P}\right)^{2}}$ \\
\hline Turton and Levenspiel (1986) & $R e_{P}<2.6 \times 10^{5}$ & $C_{D}=\frac{24}{R e_{P}}\left(1+0.173 R e_{P}^{0.657}\right)+\frac{0.413}{1+16,300 R e_{P}^{-1.09}}$ \\
\hline Khan and Richardson (1978) & $0.01<R e_{P}<3 \times 10^{5}$ & $C_{D}=\left(2.25 R e_{P}^{-0.31}+0.36 R e_{P}^{0.06}\right)^{3.45}$ \\
\hline Haider and Levenspiel (1989) & $R e_{P}<2.6 \times 10^{5}$ & $C_{D}=\frac{24}{R e_{P}}\left(1+0.1806 R e_{P}^{0.6459}\right)+\frac{0.4251}{1+\left(\frac{6880.95}{R e_{P}}\right)}$ \\
\hline Brown and Lawler (2003) & $R e_{P}<2 \times 10^{5}$ & $C_{D}=\frac{24}{R e_{P}}\left(1+0.15 R e_{P}^{0.681}\right)+\frac{0.407}{1+\left(\frac{8710}{R e_{P}}\right)}$ \\
\hline
\end{tabular}


Table (2.7-2). Drag coefficient correlations for non-spherical particles

\begin{tabular}{|c|c|c|}
\hline References & $R e_{n P}$ range & Correlation \\
\hline \multirow[t]{2}{*}{ Haider and Levenspiel (1989) } & $R e_{n p}<25,000$ & $C_{D}=\frac{24}{R e_{n p}}\left[1+\exp \left(2.3288-6.4581 \psi+2.4486 \psi^{2}\right) R e_{n p}^{0.0964+0.5565 \psi}\right]$ \\
\hline & $0.026<\psi<1.0$ & $+\frac{R e_{n p} \exp \left(4.905-13.8944 \psi+18.4222 \psi^{2}-10.2599 \psi^{3}\right)}{R e_{n p}+\exp \left(1.4681+12.2584 \psi-20.7322 \psi^{2}+15.8855 \psi^{3}\right)}$ \\
\hline \multirow[t]{2}{*}{ Ro and Neethling (1990) } & $15<R e_{n p}<87$ & $C_{D}=\frac{24 \aleph}{R e_{P}}+21.55 S_{F}^{2} R e_{n P}^{-0.518}$ \\
\hline & $S_{F}=d_{A} / d_{v}<1$ & \\
\hline \multirow[t]{2}{*}{ Thompson and Clark (1991) } & $R e_{n P}<10^{4}$ & 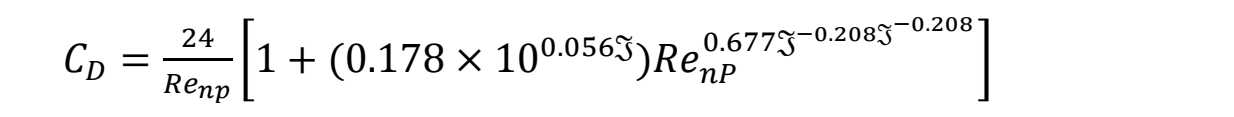 \\
\hline & $\mathfrak{J}=\frac{C_{D}}{C_{D s}}$ & $+\frac{R e_{n P}(0.101+0.366 \mathfrak{I})}{R e_{n P}+5732.1 \mathfrak{J}^{-1.96}}$ \\
\hline \multirow[t]{2}{*}{ Swamee and Ojha (1991) } & $1<R e_{n P}<10^{4}$ & \\
\hline & $0.3<C o<1.0$ & $C_{D}=\frac{48.5}{\left(1+4.5 C o^{0.35}\right)^{0.8} R e_{n P}^{0.64}}+\frac{1}{C o^{18}+1.05 C o^{0.8}}\left(\frac{R e_{n P}}{R e_{n P}+100+100}\right.$ \\
\hline
\end{tabular}

Ganser (1993)

$$
\begin{aligned}
R e_{n P} K_{1} K_{2}<10^{5} & \frac{C_{D}}{K_{2}}=\frac{24}{R e_{n P} K_{1} K_{2}}\left[1+0.1118\left(R e_{n P} K_{1} K_{2}\right)^{0.6567}\right]\left(\frac{0.4305}{1+\left(3305 / R e_{n P} K_{1} K_{2}\right)}\right)^{0.32} \\
0.026<\psi<1.0 & K_{1}=\left[\left(\frac{1}{3}\right)+\left(\frac{2}{3}\right) \psi^{-0.5}\right]^{-1}, \text { for isometric particles } \\
K_{2} & =10^{1.8148(-\log \psi)^{0.5743}}
\end{aligned}
$$


Chien (1994)

$R e_{n P} \leq 5000$

$0.2 \leq \psi \leq 1.0$

Hartman et al. (1994)

$$
\begin{aligned}
& 10^{-2}<R e_{n P}<16,000 \\
& 0.67<\psi<1.0
\end{aligned}
$$

Xie and Zhang (2001)

$$
0.15<R e_{n P}<1500
$$

$$
0.8<\frac{d_{A}}{d_{v}}<1.5
$$$$
0.4<C<1.0
$$

$$
\log C_{D}\left(R e_{n P}, \psi\right)=\log C_{D}\left(R e_{P}, 1\right)+P\left(R e_{n P}, \psi\right)
$$$$
P\left(R e_{n P}, \psi\right)=-0.03874(1-\psi) \log R e_{n P}+0.09238(1-\psi)\left(\log R e_{n P}\right)^{2}
$$$$
+0.06003(1-\psi)\left(\log R e_{n P}\right)^{3}+0.01005(1-\psi)\left(\log R e_{n P}\right)^{4}
$$$$
-0.003571(1-\psi)\left(\log R e_{n P}\right)^{5}+0.005697(1-\psi)^{2}\left(\log R e_{n P}\right)^{5}
$$

$$
C_{D}=\left(\frac{30}{R e_{n P}}\right)+67.289 \exp (-5.03 \psi)
$$

$$
C_{D}=\frac{24}{R e_{n P}} \frac{d_{A}}{d_{v}}\left[1+\frac{0.15}{\sqrt{C}}\left(\frac{d_{A}}{d_{v}} R e_{n P}\right)^{0.678}\right]+\frac{0.42\left(d_{A} / d_{v}\right)^{2}}{\sqrt{C}\left(1+4.25 \times 10^{4}\left(R e_{n P} d_{A} / d_{v}\right)^{-1.16}\right)}
$$

where $R e_{n P}=$ particle Reynolds number of non-spherical particle

$$
\psi=\text { sphericity }
$$




\subsubsection{Lift Force}

Lift force is a net resultant force acting on a body in the direction perpendicular to the relative motion of the moving fluid (Mott, 2005). It is developed due to difference in pressures on opposite sides of a body while flowing fluid passes the body. Lift force is not necessarily always vertical. Two types of lift forces have been reported in the literature. They are: (a) Saffman lift force and (b) Magnus lift force.

\subsubsection{Saffman Lift Force}

The development of pressure distributions on a body, due to its rotation caused by velocity gradients, results in Saffman lift force. Lower pressure develops on the side with higher velocity and higher pressure develops on the other side with lower velocity to cause lift force (Crowe et al., 1998).

Saffman (1965) developed the following correlation to find the magnitude of this lift force $\left(F_{\text {Saff }}\right)$ :

$$
F_{\text {Saff }}=1.61 \mu_{c} d_{P}\left|\boldsymbol{u}_{c}-\boldsymbol{u}_{P}\right| \sqrt{R e_{G}} \frac{1}{V_{P} \rho_{p}}
$$

where $R e_{G}$ represents the shear Reynolds number and can be expressed as:

$$
R e_{G}=\frac{d_{P}^{2}}{v_{c}} \frac{d u_{c}}{d y}
$$

The above equation can be arranged and expressed in the following form (Asakura et al., 1997):

$$
\boldsymbol{F}_{\text {Saff }}=1.61 d_{P}^{2}\left[\left(\boldsymbol{u}_{c}-\boldsymbol{u}_{P}\right)\right]\left[\rho_{c} \mu_{c}\left|\frac{d \boldsymbol{u}_{c}}{d y}\right|\right]^{1 / 2} \frac{d \boldsymbol{u}_{c} / d y}{\left|d \boldsymbol{u}_{c} / d y\right|} \frac{1}{V_{P} \rho_{P}}
$$


If $\left(\boldsymbol{u}_{c}-\boldsymbol{u}_{P}\right)$ becomes negative, a lift force is developed towards the lower velocity side of the fluid and vice-versa. The above presented equations (for lift forces) are only applicable when $R e_{P} \ll \sqrt{R e_{G}}$.

Mei (1992) proposed the following empirical correlation for higher particle Reynolds number:

$$
\frac{F_{L}}{F_{\text {Saff }}}=\left(1-0.3314 \beta^{\frac{1}{2}}\right) \exp \left(\frac{-R e_{P}}{10}\right)+0.3314 \beta^{1 / 2} \quad R e_{P} \leq 40
$$

and $\frac{F_{L}}{F_{\text {Saff }}}=0.0524\left(\beta R e_{P}\right)^{1 / 2} \quad R e_{P}>40$

where $\quad \beta=\frac{R e_{G}}{2 R e_{P}} \quad 0.005<\beta<0.4$

\subsubsection{Magnus Lift Force}

Rotation of particles caused by, the collision of particles and impact between particles and wall, is responsible for the development of this force. Angular velocity of the particles $\left(\boldsymbol{\omega}_{P}\right)$ divert them perpendicular to the rotational axis.

Rubinow and Keller (1961) proposed the following equation for Magnus lift force $\left(\boldsymbol{F}_{M}\right)$ per unit mass of a body $\left(m_{P}\right)$ as:

$$
\boldsymbol{F}_{M}=\frac{\pi}{8} d_{P}^{3} \rho_{c}\left[\boldsymbol{\omega}_{P} \times\left(\boldsymbol{u}_{P}-\boldsymbol{u}_{c}\right)\right] \frac{1}{V_{P} \rho_{p}}
$$


The above equation can be expressed in the other form as:

$$
\frac{\boldsymbol{F}_{M}}{m_{P}}=-\frac{3}{4}\left(\frac{\rho_{p}}{\rho_{c}}\right)\left[\begin{array}{c}
\omega_{P y}\left(w_{P}-u_{z}\right)-\omega_{P z}\left(v_{P}-u_{y}\right) \\
\omega_{P z}\left(u_{P}-u_{x}\right)-\omega_{P x}\left(w_{P}-u_{y}\right) \\
\omega_{P x}\left(v_{P}-u_{z}\right)-\omega_{P y}\left(v_{P}-u_{x}\right)
\end{array}\right]
$$

where $=\left(\begin{array}{l}u_{x} \\ u_{y} \\ u_{z}\end{array}\right), \boldsymbol{u}_{P}=\left(\begin{array}{l}u_{P} \\ v_{P} \\ w_{P}\end{array}\right)$ and $\boldsymbol{w}_{\boldsymbol{P}}=\left(\begin{array}{l}\omega_{P x} \\ \omega_{P y} \\ \omega_{P Z}\end{array}\right)$ are continuous phase velocities, linear particle velocities and angular velocities, respectively.

\subsubsection{Basset (History) Force}

The history force is caused due to delay (temporal) of formation of boundary layer with change in relative acceleration of objects passing through a fluid (Crowe et al., 1998). The Basset term takes viscous effects into considerations. The Basset (history) force is commonly neglected because it is difficult to implement. The Basset force $\left(\boldsymbol{F}_{B H}\right)$ can be expressed (Crowe et al., 1998):

$$
\boldsymbol{F}_{B H}=\frac{3}{2} D^{2} \sqrt{\rho_{c} \mu_{c} \pi}\left[\int_{0}^{t} \frac{d \boldsymbol{u}_{c}-\frac{d \boldsymbol{u}_{\boldsymbol{p}}}{d t}}{\sqrt{t-t^{\prime}}} d t^{\prime}+\frac{\left(\boldsymbol{u}_{c}-\boldsymbol{u}_{P}\right)}{\sqrt{t}}\right] \frac{1}{V_{P} \rho_{p}}
$$

where $V_{P}, D, \mu_{c}$, and $t^{\prime}$ are volume of particle, vessel diameter, viscosity of continuous phase and dummy time variable, respectively. 


\subsection{Computational Fluid Dynamics (CFD)}

Computational fluid dynamics (CFD) is a very efficient and effective tool to get detailed insight information about the complex fluid flow behaviour in less time and materials. It should be noted that CFD is not replacement for experimental measurements but instead enhances the knowledge about the flow behaviour to extract more details. CFD in case of mixing tank, can be utilized to retrieve detailed information about velocity and concentration profiles. CFD gives a qualitative (most often) and sometimes a quantitative assessment of fluid flow by making use of (a) mathematical models (b) numerical analysis and (c) reliable and suitable software tool (Fergizer and Peric, 1996; Wesseling, 2001). Although CFD cannot replace experimental measurements entirely, it can substantially reduce the number of experimental measurements and costs involved.

The number of steps involved in analysis using CFD are: (a) statement of problems to be solved (b) use of suitable mathematical models (c) generation of mesh (d) time and space discretization (approximation of temporal and spatial derivatives) (e) strategy for iterative solution (f) simulations using CFD software (g) analysis to retrieve desired results from post processing and (h) verification and validation of CFD model (Fergizer and Peric, 1996; Wesseling, 2001). It is very important to verify the results obtained from CFD because there may be various errors (local and global error, discretization error, programming error, convergence error, physical approximation error, and usage error) arisen during the simulation process. Verification and validation of CFD models can be done by examining convergence, consistency, grid independency and comparing the computational result with experimental one. It is imperative to understand different approaches in modeling of fluid flow in suspensions. There are basically two approaches: (a) Eulerian approach and (b) Lagrangian approach. These two approaches are first described for single phase flow system and then for multi-phase (solid-liquid) flow system.

\subsubsection{Eulerian and Lagrangian Approach for Single-Phase Flow}

In Eulerian approach of fluid flow, the emphasis is on defining a fixed (unchanging) control volume through which the flowing fluid passes. In this approach, all the fluid flow properties such as velocity, acceleration, pressure are identified as fields inside this control volume and each of this property is represented with respect to space and time within this volume. In this description of flow, tracking of individual fluid particle is not done. 
In Lagrangian approach of fluid flow, movement of control volume takes place with the velocity equal to that of continuous fluid stream velocity inside the fluid. In this description of flow, each particle is identified and its position, velocity etc. is described with respect to time. However, it is extremely difficult to identify and track each individual particle because of complex nature of fluid flow. Hence, application of Lagrangian method is limited to the lower particle concentrations.

The continuity equation can be expressed in vectorial notation for Eulerian description of fluid flow by considering a fixed control volume (Bird et al., 2002):

$$
\frac{\partial \rho}{\partial t}=-\nabla \cdot \rho \boldsymbol{V}
$$

where $\rho$ and $\boldsymbol{V}$ are density of fluid and fluid stream velocity, respectively.

A substantial derivative is used to express the continuity equation for a control volume moving with the fluid stream velocity as:

$$
\frac{D \rho}{D t}=-\rho \nabla \cdot \boldsymbol{V}
$$

The above equations can be expressed as:

$$
\frac{\partial \rho}{\partial t}=-\boldsymbol{V} \cdot \nabla \rho-\rho \nabla \cdot \boldsymbol{V}
$$


The momentum equation (in vector notation) for the Eulerian description of fluid flow can be written as:

$$
\frac{\partial(\rho \boldsymbol{V})}{\partial t}=-\nabla \cdot \boldsymbol{\tau}-\nabla \cdot \rho \boldsymbol{V} \boldsymbol{V}-\nabla p+\rho \boldsymbol{g}
$$

where $\boldsymbol{\tau}$ and $p$ are stress tensor and pressure, respectively.

The momentum equation (in vector notation) for the Lagrangian description of fluid flow can be given by:

$$
\frac{\partial(D \boldsymbol{V})}{\partial t}=-\nabla \cdot \boldsymbol{\tau}-\nabla p+\rho \boldsymbol{g}
$$

\subsubsection{Selection of Suitable Approach for Modeling Solid-Liquid Suspension Flow}

For continuous fluid phase, only the Eulerian approach can be applied whereas for particle phase, either Eulerian approach or Lagrangian approach can be utilized while creating models for suspensions flow. Selection of a suitable approach for the particle (dispersed) phase significantly relies on: (a) particle size $\left(d_{P}\right)$, (b) volume fraction of the particle, (c) response time of the particle $\left(t_{P r}\right)$, and (d) characteristics time scales of the fluid phase $\left(t_{c}\right)$. The particle response time $\left(t_{P r}\right)$ can be defined as the time taken by a solid particle to show response to any variations in the fluid flow velocity $\left(V_{t}\right)$ and can be correlated in terms of the drag coefficient $\left(C_{D}\right)$ and the velocity vector (Rani et al., 2004) :

$$
t_{P r}=\left(\frac{4}{3}\right)\left(\frac{\rho_{p}}{\rho}\right) \frac{d_{P}}{C_{D} V_{t}}
$$


For extremely fine particles $\left(R e_{P}<1\right), t_{P r}$ can be expressed in the Stokes regime as:

$$
t_{P r}=\frac{\rho_{p} d_{P}^{2}}{18 \mu}
$$

where $R e_{P}, \rho_{p}, \rho$, and $\mu$ are particle Reynolds number, particle density, continuous phase density and viscosity, respectively. $t_{c}$ can be defined as the time consumed by the liquid to pass through a system of length $L$ :

$$
t_{c}=\frac{L}{V_{c}}
$$

where $V_{c}$ represents superficial velocity of the fluid. Stokes number $(S t)$ can be defined as:

$$
S t=\frac{t_{p r}}{t_{c}}
$$

The dispersed (particle) phase velocity approximately reaches the continuous (liquid) phase velocity when $S t$ is less than 0.1 because particles get enough time to show response to any variations in the fluid velocity. However, particles do not get enough time to show response to any variations in the fluid velocity thus showing minor fluctuations in their velocity when $S t$ is more than 10 . 
When size of the particle is very small $\left(\varnothing \approx 10^{-4}\right)$, relative motion between the particles and the continuous liquid phase does not exist. Hence, the system can be treated as single phase flow and the Eulerian approach can be used for modeling this kind of suspension flow. When size of the particle is large enough, particles should be treated as different phase (dispersed phase) and models based on two-phase flow should be employed. The suitable modeling approach for this kind of system is Eulerian-Eulerian approach. If particles of different sizes are present in the system, particle velocity and distribution of the solid volume fraction should be identified and characterised for each size. For modeling of this kind of flow system, Eulerian approach for the liquid phase and Lagrangian approach for the particle phase should be adopted and the modeling approach itself is called as Eulerian-Lagrangian approach. When size of the particle is very large, none of the above mentioned approaches are suitable for modeling the flow system. A recently developed approach known as Superparticle Lagrangian method can be utilized in modeling the particle (dispersed) phase. In this approach, particles with similar characteristics are considered in one group and their location is determined by using Newton's equation of motion.

\subsubsection{Eulerian-Eulerian Approach}

In this description of fluid flow, the particle (dispersed) phase is considered as a fluid (continuous) phase with the assumption that the particle phase interpenetrates and interacts with the liquid phase (Gidaspow, 1994). In this model, equations of continuity and motion are solved for both (continuous and dispersed) phases while sharing a single pressure between them. Equations of continuity and momentum thus obtained should be averaged with respect to time or space. The ensemble equations (empirical most of the times) should reflect the particle interactions in the system. While modeling the flow system for this study, time averaged Navier-Stokes equations will be employed. For determination of drag coefficient, empirical correlation proposed by Gidaspow et al. (1992) for two phase (solid-liquid) interactions will be used. Particles are assumed to be of same size (one size) to prevent any complexion arisen by considering multi-sized particles.

Continuity equation can be given by (Fluent Inc., 2006): 


$$
\frac{\partial\left(a_{q} \rho_{q}\right)}{\partial t}+\nabla \cdot\left(a_{q} \rho_{q} \vec{u}_{q}\right)=0
$$

The momentum equation can be written as (Fluent Inc., 2006):

$$
\begin{aligned}
& \frac{\partial}{\partial t}\left(a_{q} \rho_{q} \vec{u}_{q}\right)+\nabla \cdot\left(a_{q} \rho_{q} \vec{u}_{q} \vec{u}_{q}\right)= \\
& -a_{q} \nabla p+\nabla \cdot\left(\overline{\overline{\tau_{q}}}\right)+a_{q} \rho_{q} \vec{g}+\left(\vec{F}_{D q}+\vec{F}_{B q}+\vec{F}_{L q}+\vec{F}_{v m q}\right)
\end{aligned}
$$

where $\overline{\overline{\tau_{q}}}$ represents the $q^{\text {th }}$ phase stress tensor and can be expressed as:

$$
\overline{\overline{\tau_{q}}}=a_{q}\left(\mu_{q}+\mu_{q t}\right)\left(\nabla \vec{u}_{q}+\nabla \vec{u}_{q}^{T}\right)-\frac{2}{3} a_{q} \rho_{q} k_{q} \overline{\bar{I}}
$$

where subscript $q$ denotes phase $q . k_{q}, \mu_{q t}$, and $\mu_{q}$ are turbulent kinetic energy, turbulent viscosity and shear viscosity respectively. $\vec{F}_{D q}, \vec{F}_{L q}, \vec{F}_{B q}$, and $\vec{F}_{v m q}$ are drag force, lift force, buoyant force and added mass force, respectively. $\overline{\bar{I}}$ is unit tensor. Drag force can be given by:

$$
\vec{F}_{D}=\sum K_{l q}\left(\vec{u}_{l}-\vec{u}_{q}\right)
$$

where $K_{l q}$ represents exchange coefficient between $q^{\text {th }}$ phase and the liquid phase. When $\frac{\rho_{p}}{\rho_{l}}>2$ , drag force and forces interacting between the particle phase and fluid phase become dominant in comparison to lift force, added mass force and buoyant force. $\frac{\rho_{p}}{\rho_{l}}$ represents ratio of particle to liquid density. 


\subsubsection{Solid-Liquid Exchange Coefficient}

$K_{l q}$ for dispersed (particle) phase can be denoted as $K_{p l}$ and expressed as (Fluent Inc., 2006):

$$
K_{p l}=\frac{a_{p} \rho_{p}}{\tau_{p}} f
$$

where $K_{p l}=K_{l p} \cdot a_{p}, \rho_{p}$, and $f$ are particle volume fraction, particle density and drag function, respectively. $\tau_{p}$ is particle relaxation time and can be defined as:

$$
\tau_{p}=\frac{\rho_{p} d_{p}^{2}}{18 \mu_{l}}
$$

where $d_{p}$ and $\mu_{l}$ are particle diameter and liquid phase viscosity, respectively. Syamlal and O’Brien (1989) expressed the drag function $(f)$ as:

$$
f=\frac{C_{D} R e_{r} a_{l}}{24 u_{t p}^{2}}
$$

where $C_{D}, R e_{r}$, and $u_{t p}$ are drag coefficient, relative Reynolds number and particle terminal velocity, respectively. Dalla (1948) proposed the following empirical correlation for $C_{D}$ :

$$
C_{D}=\left[0.63+\frac{4.8}{\sqrt{R e_{r} / u_{t p}}}\right]^{2}
$$


$R e_{r}$ can be defined as:

$$
R e_{r}=\frac{\rho_{l} d_{p}\left|\vec{u}_{p}-\vec{u}_{l}\right|}{\mu_{l}}
$$

Where subscripts $p$ and $l$ represent the particle and liquid phase respectively. $K_{p l}$ finally changes to:

$$
K_{p l}=\frac{3 a_{p} a_{l} \rho_{l}}{4 u_{t p} 2 d_{p}} C_{D} \frac{R e_{r}}{u_{t p}}\left|\vec{u}_{p}-\vec{u}_{l}\right|
$$

Garside and Al-Dibouni (1977) proposed the following empirical correlation for solid particle terminal velocity:

$$
u_{t p}=0.5\left(A-0.06 R e_{r}+\sqrt{\left(0.06 R e_{r}\right)^{2}+0.12 R e_{r}(2 B-A)+A^{2}}\right)
$$

where

$$
\begin{aligned}
& A=a_{l}^{4.14} \\
& B=0.8 a_{l}^{1.28} \quad \text { if } a_{l} \geq 0.85
\end{aligned}
$$

and

$$
B=a_{l}^{2.65} \quad \text { if } a_{l}<0.85
$$


Wen and Yu (1966) proposed the following correlation for $K_{p l}$ :

$$
K_{p l}=\frac{3 a_{p} a_{l} \rho_{l}}{4 d_{p}} C_{D}\left|\vec{u}_{p}-\vec{u}_{l}\right| a_{l}^{2.65} \text { if } a_{l}>0.8
$$

and

$$
K_{p l}=150 \frac{a_{p}\left(1-a_{l}\right) \mu_{l}}{d_{p}^{2} a_{p}}+1.75\left|\vec{u}_{p}-\vec{u}_{l}\right| \frac{a_{p} \rho_{l}}{d_{p}} \quad \text { if } a_{l} \leq 0.8
$$

Gidaspow et al. (1992) developed the following empirical equation for the dense fluidized bed:

$$
C_{D}=\frac{24}{a_{l} R e_{r}}\left[1+0.15\left(a_{l} R e_{r}\right)^{0.687}\right] \text { if } R e_{r} \leq 1000
$$

and

$$
C_{D}=0.44 \text { if } R e_{r}>1000
$$

\subsubsection{Eulerian-Lagrangian Approach (Dispersed Phase Model)}

In this approach, tracking of motion of an individual particle is done by solving equation of motion for each particle on an individual basis. However, for liquid phase (continuous phase) Eulerian method is employed. The momentum transport is assessed by calculating the difference in momentum when each particle travels through a control element. 
In this approach, position of a particle $\left(\boldsymbol{X}_{\boldsymbol{P}}\right)$ is written in terms of particle velocity vector $\left(\boldsymbol{V}_{P}\right)$ as:

$$
\frac{d X_{P}}{d t}=V_{P}
$$

$\boldsymbol{V}_{P}$ is evaluated after equation of motion is solved. Ferry and Balachandar (2001) derived the following equations of motion (differential form):

$$
\begin{gathered}
m_{P} \frac{d \boldsymbol{V}_{P}}{d t}=6 \pi d_{P} \mu\left(\boldsymbol{V}_{c}-\boldsymbol{V}_{P}\right)+m_{c} \frac{D \boldsymbol{V}}{D t}+\frac{1}{2} m_{c}\left(\frac{D \boldsymbol{V}_{c}}{D t}-\frac{D \boldsymbol{V}_{P}}{D t}\right)+\left(m_{P}-m_{c}\right) g \\
+\left(\frac{6 \pi d_{P}^{2} \mu}{\left(\frac{\mu}{\rho_{P}}\right)^{1 / 2}}\right) \frac{d^{1 / 2}}{d t^{1 / 2}}\left(\boldsymbol{V}_{c}-\boldsymbol{V}_{P}\right)+\left(\frac{9 J_{\infty}}{\pi}\right) d_{P}^{2}\left(\frac{\mu \rho}{|\omega|}\right)^{1 / 2}\left(\boldsymbol{V}_{c}-\boldsymbol{V}_{P}\right) \times \omega
\end{gathered}
$$

where $m_{c}, m_{P}$, and $\boldsymbol{V}_{c}$ are mass of fluid (continuous) phase, mass of particle and velocity vector for continuous phase, respectively. The six terms at the right hand side of the above equation are Stokes drag force (first term), force due to fluid acceleration (second term), added mass force (third term), gravitational force (fourth term), history (Basset) force (fifth term), and Saffman lift force (sixth term), respectively. It should be noted that the above equation is only applicable for unsteady state condition in laminar regime (Stokes regime). $\omega$ and $J_{\infty}$ represent vorticity and Saffman lift function respectively. Ferry and Balachandar (2001) took Saffman lift function value as:

$$
J_{\infty} \approx 2.255 \quad \text { for } \quad \text { Stokes regime }
$$

The above equation can be written as: 


$$
\frac{d \boldsymbol{V}_{P}}{d t}=\frac{1}{t_{P r}}\left(\boldsymbol{V}_{c}-\boldsymbol{V}_{P}\right)+\beta \frac{D \boldsymbol{V}_{\boldsymbol{c}}}{D t}+(1-\beta) g+\left(\frac{\beta}{t_{P r}}\right)^{1 / 2} \ell\left[\boldsymbol{V}_{c}-\boldsymbol{V}_{P}\right]
$$

where $\beta$ is a density parameter and can be expressed as:

$$
\beta=\frac{3}{2\left(\frac{\rho_{p}}{\rho_{c}}\right)+1}
$$

and $\ell\left[\boldsymbol{V}_{c}-\boldsymbol{V}_{P}\right]$, which is known to be linear operator can be expressed as:

$$
\ell\left[\boldsymbol{V}_{c}-\boldsymbol{V}_{P}\right]=\left(\sqrt{3} \frac{d^{1 / 2}}{d^{1 / 2}}\left(\mathbf{V}_{c}-\mathbf{V}_{\mathrm{P}}\right)+\frac{3 \sqrt{3} J_{\infty}}{2 \pi^{2} \sqrt{|\omega|}}\left(\boldsymbol{V}_{c}-\boldsymbol{V}_{P}\right) \times \omega\right)
$$

$\beta$ can cover particle of all sizes (Rani and Balachandar, 2004):

$$
\begin{array}{lll}
\beta \approx 0 & \text { if } \frac{\rho_{p}}{\rho} \gg 1 & \text { (dense particles) } \\
\beta=3 & \text { if } \frac{\rho_{p}}{\rho} \ll 1 & \text { (light particles) }
\end{array}
$$

\subsubsection{Fast or Equilibrium Eulerian Approach}

The fast or equilibrium approach is recently developed for multiphase flows. This approach is derived to obtain distribution of velocity (Eulerian form) of the dispersed (particle) phase 
$\boldsymbol{V}_{P}(t, \boldsymbol{X})$ for very small particle relaxation time $\left(t_{P r}\right)$. According to this approach, the velocity of particle can be expressed as (Pekker and Helvaci, 2008):

$$
\boldsymbol{V}_{P}=\boldsymbol{V}_{c}+(1-\beta)\left(-\boldsymbol{a} t_{P r}+\sqrt{\beta} \ell \boldsymbol{a} t_{P r}^{3 / 2}+\left(\frac{D a}{D t}+\boldsymbol{a} . \nabla \boldsymbol{V}_{c}-\beta \ell^{2} \boldsymbol{a}\right) t_{P r}^{2}\right)+O\left(t_{P r}^{5 / 2}\right)
$$

where $\boldsymbol{a}$ comprises of the substantial derivative of the continuous phase and gravity terms as:

$$
a=\frac{D V_{c}}{D t}-g
$$

When force due to gravity is prevalent, the above equation can be expressed as:

$$
\begin{aligned}
& \boldsymbol{V}_{P}=\boldsymbol{V}_{c}+\boldsymbol{V}_{t}-\sqrt{\beta} \boldsymbol{e} \boldsymbol{V}_{t} t_{P r}^{\frac{1}{2}}-\left((1-\beta) \frac{D V_{c}}{D t}+\frac{D V_{t}}{D t}\right) t_{P r} \\
& -\left(\boldsymbol{V}_{t} . \nabla\left(\boldsymbol{V}_{c}+\boldsymbol{V}_{t}\right)-\beta \ell^{2} \boldsymbol{V}_{t}\right) t_{P r}+O\left(t_{P r}^{3 / 2}\right)
\end{aligned}
$$

Generally acceleration due to gravity, as a result, $\boldsymbol{V}_{t}$ becomes approximately constant with respect to position and time. Thus the history force components of $\boldsymbol{\ell} \boldsymbol{V}_{t}$ approaches zero and Saffman lift forces become effective:

$$
\boldsymbol{V}_{P}=\boldsymbol{V}_{c}+\boldsymbol{V}_{t}-\frac{3 J_{\infty}}{2 \pi^{2}} \sqrt{\frac{3 \beta t_{P r}}{|\omega|}} \omega \times \boldsymbol{V}_{t}+O\left(t_{P r}\right)
$$


For very dense particles, $\beta$ approaches zero and Saffman lift force does not have effect:

$$
\boldsymbol{V}_{P}=\boldsymbol{V}_{c}+\boldsymbol{V}_{t}-\left(\frac{D V_{c}}{D t}+\boldsymbol{V}_{t} . \nabla \boldsymbol{V}_{c}\right) t_{P r}+O\left(t_{P r}^{2}\right)
$$

For very small particles, effects of Saffman lift and History forces can be ignored and $\ell$ can be neglected:

$$
\boldsymbol{V}_{P}=\boldsymbol{V}_{c}+(1-\beta)\left(-\boldsymbol{a} \boldsymbol{t}_{P r}+\left(\frac{D a}{D t}+\boldsymbol{a} \cdot \nabla \boldsymbol{V}_{c}\right)\right) t_{P r}^{2}+O\left(t_{P r}^{3}\right)
$$

When the effects of drag force, gravity and collision are excluded, the above equation can be written as (Rani and Balachandar, 2004):

$$
\boldsymbol{V}_{P}(t, \boldsymbol{X}) \approx \boldsymbol{V}_{P, e q}=\boldsymbol{V}_{c}-(1-\beta) t_{P r} \frac{D V_{c}}{D t}+O\left(t_{P r}^{2}\right)
$$

\subsubsection{Turbulence Models}

When solid particles in solid-liquid mixing operations are agitated to increase the local mixing quality, fluctuations developed due to turbulence need to be taken into considerations while creating a flow model to describe the system. It is well understood that turbulence needs very high energy to mitigate the effect of additional drag force but it is inevitable for effective mixing and distribution (Kleinstreuer, 2003). In case of turbulent flow, velocities may keep on rising or falling and as a result, the equation of motion, not the equation of continuity, will have an additional term to describe these fluctuations with respect to mean velocity. 
As these turbulent fluctuations may be of small scales and high intensity, computation of these fluctuations for simple engineering problems can be cumbersome and expensive while simulation. To get rid of the fluctuations of small scales, equations for instantaneous changes in motion can be time-smoothed or modified. The manipulated (modified) equations comprise of unknown parameters. Hence the turbulence model is required to evaluate these unknown parameters in terms of known variables (Fluent, 2006).

In Reynolds time-smoothed (averaged) method, variables such as velocity, pressure are broken up into two components: (a) mean and (b) fluctuating. For Z-component of the velocity, Reynolds decomposition can be given by (Bird et al., 2002):

$$
v_{z}=\bar{v}_{z}+v_{z}^{\prime}
$$

where $\bar{v}_{z}$ represents mean value and $v_{z}^{\prime}$ represents fluctuating component. The time averaged velocity $\left(\bar{v}_{z}\right)$ can be obtained by taking a time average over turbulent fluctuations $(s)$ as:

$$
\bar{v}_{z}=\frac{1}{t_{0}} \int_{t-\left(\frac{1}{2}\right) t_{0}}^{t+\left(\frac{1}{2}\right) t_{0}} v_{z}(s) d s
$$

The turbulent intensity can be utilised to assess the degree of fluctuations. The equation of continuity can be expressed as:

$$
\frac{\partial}{\partial x}\left(\bar{v}_{x}+v_{x}^{\prime}\right)+\frac{\partial}{\partial y}\left(\bar{v}_{y}+v_{y}^{\prime}\right)+\frac{\partial}{\partial z}\left(\bar{v}_{z}+v_{z}^{\prime}\right)=0
$$

The momentum equation for $\mathrm{x}$-component can be expressed as: 


$$
\begin{gathered}
\rho\left(\bar{v}_{x}+v_{x}^{\prime}\right)=-\frac{\partial}{\partial x}\left(\bar{p}+p^{\prime}\right)-\left(\frac{\partial}{\partial x} \rho\left(\bar{v}_{x}+v_{x}^{\prime}\right)\left(\bar{v}_{x}+v_{x}^{\prime}\right)+\frac{\partial}{\partial y} \rho\left(\bar{v}_{y}+v_{y}^{\prime}\right) \quad\left(\bar{v}_{x}+\right.\right. \\
\left.\left.v_{x}^{\prime}\right)+\frac{\partial}{\partial z} \rho\left(\bar{v}_{z}+v_{z}^{\prime}\right)\left(\bar{v}_{x}+v_{x}^{\prime}\right)\right)+\mu \nabla^{2}\left(\bar{v}_{x}+v_{x}^{\prime}\right)+\rho g_{x}
\end{gathered}
$$

The momentum equations for $\mathrm{y}$ - and z-components can be expressed similarly. The above equations can be time averaged as (Bird et al. 2002):

$$
\frac{\partial}{\partial x} \bar{v}_{x}+\frac{\partial}{\partial y} \bar{v}_{y}+\frac{\partial}{\partial z} \bar{v}_{z}=0
$$

and

$$
\begin{gathered}
\frac{\partial}{\partial t} \rho \bar{v}_{x}=-\frac{\partial}{\partial x} \bar{p}-\left(\frac{\partial}{\partial x} \rho \bar{v}_{x} \bar{v}_{x}+\frac{\partial}{\partial y} \rho \bar{v}_{y} \bar{v}_{x}+\frac{\partial}{\partial z} \rho \bar{v}_{z} \bar{v}_{x}\right)- \\
\left(\frac{\partial}{\partial x} \rho \overline{v_{x}^{\prime} v_{x}^{\prime}}+\frac{\partial}{\partial y} \rho \overline{v_{y}^{\prime} v_{x}^{\prime}}+\rho \overline{v_{z}^{\prime} v_{x}^{\prime}}\right)+\mu \nabla^{2} \bar{v}_{x}+\rho g_{x}
\end{gathered}
$$

$y-$ and z-components of the momentum equation can be written similarly. These equations are similar to equations of continuity and momentum, except, the variables are time-smoothed (averaged). These equations are known to be Reynolds averaged (time-smoothed) Navier Stokes equations of change. Additional terms now obtained, describe the effects of turbulent fluctuations. The turbulent momentum stress tensor can be expressed as:

$$
\bar{\tau}_{x x}^{(t)}=\rho \overline{v_{x}^{\prime} v_{x}^{\prime}}, \bar{\tau}_{x y}^{(t)}=\rho \overline{v_{x}^{\prime} v_{y}^{\prime}}, \bar{\tau}_{x z}^{(t)}=\rho \overline{v_{x}^{\prime} v_{z}^{\prime}} \text { and so on }
$$


The above mentioned quantities are generally called as Reynolds stresses. The time-averaged viscous stress tensors can be expressed as:

$$
\bar{\tau}_{x x}^{(v)}=-2 \mu \frac{\partial \bar{v}_{x}}{\partial x}, \quad \bar{\tau}_{x y}^{(v)}=-\mu\left(\frac{\partial \bar{v}_{y}}{\partial x}+\frac{\partial \bar{v}_{x}}{\partial y}\right) \text { and so on }
$$

By incorporating the above mentioned quantities, the equations of continuity and motion (in vector-tensor form) can be expressed as (Bird et al., 2002):

$$
\begin{gathered}
(\boldsymbol{\nabla} \cdot \overline{\mathbf{v}})=0 \quad \text { and } \quad\left(\boldsymbol{\nabla} \cdot \mathbf{v}^{\prime}\right)=0 \\
\frac{\partial}{\partial t} \rho \overline{\mathbf{v}}=-\boldsymbol{\nabla} \bar{p}-[\boldsymbol{\nabla} \cdot \rho \overline{\mathbf{v}} \overline{\mathbf{v}}]-\left[\boldsymbol{\nabla} \cdot\left(\overline{\mathbf{\tau}}^{(v)}+\overline{\mathbf{\tau}}^{(t)}\right)\right]+\rho \mathbf{g}
\end{gathered}
$$

where $\overline{\mathbf{\tau}}^{(v)}$ and $\overline{\mathbf{\tau}}^{(t)}$ are viscous stress tensor and turbulent stress tensor respectively. Boussinesq hypothesis is employed to correlate $\overline{\mathbf{\tau}}^{(t)}$ with respect to velocity gradient (mean):

$$
\overline{\mathbf{\tau}}^{(t)}=\mu^{(t)}\left(\frac{\partial v_{i}}{\partial x_{j}}+\frac{\partial v_{j}}{\partial x_{i}}\right)-\frac{2}{3}\left(\rho k+\mu^{(t)} \frac{\partial v_{k}}{\partial x_{k}}\right) \delta_{i j}
$$

where $\mu^{(t)}$ denotes eddy (turbulent) viscosity. In case of high turbulent flow, eddy viscosity becomes dominant, as a result, the effects of laminar viscosity can be negligible. $k-\varepsilon$ model is most commonly used turbulent model. This model employs two equations (additional): (a) one equation for kinetic energy of turbulence $(k)$ and (b) one equation for turbulent dissipation rate $(\varepsilon)$. The equation for kinetic energy of turbulence $(k)$ can be given by: 


$$
\frac{\partial(\rho k)}{\partial t}+\frac{\partial}{\partial x_{i}}\left(\rho \boldsymbol{v}_{i} k\right)=\frac{\partial}{\partial x_{i}}\left(\mu+\frac{\mu^{(t)}}{\sigma_{k}}\right) \frac{\partial k}{\partial x_{i}}+G_{k}+G_{b}-\rho \varepsilon-Y_{m}
$$

and equation for dissipation rate of turbulence $(\varepsilon)$ is:

$$
\frac{\partial(\rho \varepsilon)}{\partial t}+\frac{\partial}{\partial x_{i}}\left(\rho \boldsymbol{v}_{\boldsymbol{i}} \varepsilon\right)=\frac{\partial}{\partial x_{i}}\left(\mu+\frac{\mu^{(t)}}{\sigma_{\varepsilon}}\right) \frac{\partial \varepsilon}{\partial x_{i}}+C_{1 \varepsilon} \frac{\varepsilon}{k}\left(G_{k}+C_{3 \varepsilon} G_{b}\right)-C_{2 \varepsilon} \rho \frac{\varepsilon^{2}}{K}-Y_{m}
$$

where $G_{k}, G_{b}$ and $Y_{m}$ are turbulent kinetic energies developed due to velocity gradient, buoyancy effect and fluctuating distension of eddies, respectively. $C_{1 \varepsilon}, C_{2 \varepsilon}, C_{3 \varepsilon}, \sigma_{k}$ and $\sigma_{\varepsilon}$ are empirical constants.

The eddy viscosity can be expressed as:

$$
\mu^{(t)}=\rho C_{\mu} \frac{K^{2}}{\varepsilon}
$$

where $C_{\mu}$ is constant, $G_{k}$ can be expressed as:

$$
G_{k}=\mu^{(t)}\left[\frac{\partial v_{i}}{\partial x_{j}}+\frac{\partial v_{j}}{\partial x_{i}}\right] \frac{\partial v_{j}}{\partial x_{i}}
$$

In solid-liquid mixing operations, the fluctuations developed due to turbulence need to be taken into considerations while creating a flow model to describe the system. There are several models 
reported in the literature which utilize Reynolds averaging method to evaluate the effect of turbulence (Joshi et al., 2011; Van den Akker, 2010). Some of them are: (a) mixing length model (zero-equation modeling), (b) Spalart-Almaras model (one-equation modeling), (c) standard $k$ - $\varepsilon$ model (two-equation modeling), (d) renormalization group $k-\varepsilon$ model (two-equation modeling), (e) realizable $k-\varepsilon$ model (two-equation modeling), (f) $k$ - $\omega$ model (two-equation modeling), (g) algebraic stress model (two-equation modeling), and (h) Reynolds stress model (seven-equation modeling). Additional partial differential equations need to be calculated for determination of eddy viscosity based on the model selected. Each of these models has merits and demerits which should be taken into account while selecting a model to be best suited for the system of interest. Various factors such as computational time, precision, complexities involved, cost and ease of operation need to be taken into considerations. The standard $k$ - $\varepsilon$ turbulence model is most frequently utilized to analyze the turbulence effects because this model is easier to use and reasonably reliable results can be obtained (Micale et al., 2000; Ranade, 2002; Spidla et al., 2005; Khopkar et al., 2006; Fradette et al., 2007; Hosseini et al., 2010b). The standard $k-\varepsilon$ model is not applicable for rotating and swirling flow conditions. For rotating and swirling flows, realizable $k-\varepsilon$ and RNG $k-\varepsilon$ models can be employed for reliable results. Unlike the standard $k-\varepsilon$ and RNG $k-\varepsilon$ models, the realizable $k-\varepsilon$ model can provide user an accurate prediction for round jet circulation. In algebraic stress model (ASM), Boussinesq hypothesis is not employed unlike $k-\varepsilon$ models. For correct prediction of extremely complex flows, the Reynolds stress model (RSM) can be used. However, computational time and cost is very high for simulation using the RSM.

Large eddy simulation (LES) provides better accuracy in comparison to RANS models for turbulent flow conditions because very small scales of eddies are also considered using sub-grids cell. However, due to high computational time and cost associated with this method, the application of this simulation technique is restricted to small agitated tank with low solid loadings (Derksen, 2003). Direct numerical simulation (DNS) is the most advanced and precise technique to get insight information about the turbulent flow conditions. In this method, equations of motion are solved on extremely fine grid at an extremely small time-step. Due to very high computational time and cost involved using DNS, this technique is limited to lab scale simulation. 


\subsubsection{Discrete Element Method (distinct element method)}

Advancements in computer programming and enhancements in computational power and speed have paved the way to simulate and model complicated particle suspensions flow with the help of distinct element method (DEM). This techniques employs Lagrangian approach to model the dispersed (particle) phase where individual distinct and interacting solid particles are assembled while modeling (Perkins and Williams, 2001). A key feature of this method is that simulation of a large quantity of interacting particles can be done even when communication network (topology) of the system is completely altered. In this technique, equations of motion are solved for the assessment of motion of discrete particle and then contact laws are added for solving forces developed due to particle interactions and collisions (Perkins and Williams, 2001). For better understanding of complex flow problems, the DEM has been coupled with other flow models for CFD formulation. The limitations of the DEM technique are: (a) applicable only for low solid concentrations, (b) not effective in simulation of non-spherical solid particles, and (c) not capable to provide quantitative description of granular flow. A brief literature review of modeling of mixing processes in solid-liquid mixing using CFD is presented in Table (2.8-1). 
Table (2.8-1). A brief literature review of modeling of mixing processes in solid -liquid mixing using CFD

\begin{tabular}{|c|c|c|c|c|}
\hline References & CFD Code & Approach Model & Objective & Findings/Comments \\
\hline $\begin{array}{l}\text { Decker \& Sommerfeld } \\
1996\end{array}$ & & Eulerian/Lagrangian & $\begin{array}{l}\text { Simulation of solid- } \\
\text { liquid flow in } \\
\text { agitated vessel to } \\
\text { study particle } \\
\text { suspension }\end{array}$ & $\begin{array}{l}\text { CFD gave satisfactory agreement } \\
\text { with experimental data, Minor effect } \\
\text { of particle size }\end{array}$ \\
\hline Kim et al., 1998 & & Lagrangian & $\begin{array}{l}\text { Modifying the } \\
\text { Navier-Stokes } \\
\text { equation for particle } \\
\text { motion }\end{array}$ & $\begin{array}{l}\text { New model for Reynolds number ( } \\
2-150) \text { and particle to fluid density } \\
\text { ratio }(5-200) \text { with better fit was } \\
\text { proposed }\end{array}$ \\
\hline Chen \& Pereira., 2000 & & $\begin{array}{l}\text { Hybrid Eulerian- } \\
\text { Lagrangian, non-linear } \\
k-\varepsilon \text { model }\end{array}$ & $\begin{array}{l}\text { Simulation of solid- } \\
\text { liquid flow system in } \\
\text { a pipe with respect to } \\
\text { velocity }\end{array}$ & $\begin{array}{l}\text { Good agreement of experimental } \\
\text { results with anisotropic turbulent } \\
\text { model, The turbulent anisotropy } \\
\text { should be taken into consideration } \\
\text { when predicting anisotropic } \\
\text { turbulent two-phase flows. }\end{array}$ \\
\hline Micale et al., 2000 & $\begin{array}{l}\text { CFX4.2 } \\
\text { Simpelec }\end{array}$ & MRF $\& k-\varepsilon$ Eulerian & $\begin{array}{l}\text { Concentration profile } \\
\text { is overestimated }\end{array}$ & $\begin{array}{l}\text { Multi fluid model and settling } \\
\text { velocity model achieved / good } \\
\text { agreement with experimental results }\end{array}$ \\
\hline Altway et al., 2001 & $\begin{array}{l}\text { Fluent 5.1, } \\
\text { ASM }\end{array}$ & $\begin{array}{l}\text { Standard } k-\varepsilon \text { turbulent } \\
\text { model }\end{array}$ & $\begin{array}{l}\text { effect of particle size } \\
\text { on three-dimensional } \\
\text { particle dispersion } \\
\text { and distribution in a } \\
\text { stirred baffled tank }\end{array}$ & $\begin{array}{l}\text { No tangible effect seen, } d_{P}=10-87 \\
\mu \mathrm{m} \text {, validate with } 87 \mu \mathrm{m} \\
\text { The algebraic slip mixture model } \\
\text { results can predict solid } \\
\text { concentration in the regions where } \\
\text { experimental measurement is } \\
\text { difficult to achieve. }\end{array}$ \\
\hline
\end{tabular}


Table (2.8-1). Continued (1)

\begin{tabular}{|c|c|c|c|c|}
\hline References & CFD Code & Approach Model & Objective & Findings/Comments \\
\hline Barrue et al., 2001 & Fluent 5 & $\begin{array}{l}\text { Black box and } k-\varepsilon \\
\text { Eulerian }\end{array}$ & $\begin{array}{l}\text { To obtain } \\
\text { concentration profiles }\end{array}$ & $\begin{array}{l}\text { Remarkable CFD results/the error } \\
\text { analysis was not done hence there is } \\
\text { no number for remarkable results }\end{array}$ \\
\hline Montante et al., 2001 & CFX4.3 & $\begin{array}{l}\text { Sliding mesh, Eulerian- } \\
\text { Eulerian, } k-\varepsilon \\
\text { turbulence model }\end{array}$ & $\begin{array}{l}\text { Particle size and rpm } \\
\text { effect on } \\
\text { concentration profile }\end{array}$ & $\begin{array}{l}k-\varepsilon \text { has good in fully turbulent, } \\
\text { RNG is good for transient }\end{array}$ \\
\hline $\begin{array}{l}\text { Ljungqvist and } \\
\text { Rasmuson., } 2001\end{array}$ & CFX4 & MRF and $k-\varepsilon$ Eulerian & $\begin{array}{l}\text { To study the effect of } \\
\text { different drag force }\end{array}$ & $\begin{array}{l}\text { Large deviation between model and } \\
\text { exp. for low density or small } \\
\text { particle, little difference between } \\
\text { drag models }\end{array}$ \\
\hline Sha et al., 2001 & CFX4.2 & MRF and $k-\varepsilon$ Eulerian & $\begin{array}{l}\text { To study and analyse } \\
\text { the effect of particle } \\
\text { size distribution }\end{array}$ & $\begin{array}{l}\text { Good agreement with } \\
\text { experimental/No comparison } \\
\text { between a unique size particle made }\end{array}$ \\
\hline $\begin{array}{l}\text { Oshinowo and Bakker., } \\
2001\end{array}$ & $\begin{array}{l}\text { Fluent } 4.52 \\
\text { EGM }\end{array}$ & MRF and $k-\varepsilon$ Eulerian & $\begin{array}{l}\text { To study the effect of } \\
\text { solid loading and } \\
\text { cloud height at } N_{j s}\end{array}$ & $\begin{array}{l}\text { Very good agreement, scale up } \\
\text { criteria had discrepancies with } \\
\text { literature }\end{array}$ \\
\hline Ranade et al., 2002 & Fluent Quick & $\begin{array}{l}\text { Black box and } k-\varepsilon \\
\text { Eulerian }\end{array}$ & $\begin{array}{l}\text { Velocity profile near } \\
\text { and far from impeller }\end{array}$ & $\begin{array}{l}\text { Both spot showed good agreement } \\
\text { with experimental results }\end{array}$ \\
\hline Kaufmann et al., 2002 & & $\begin{array}{l}\text { Both Eulerian and } \\
\text { Lagrangian }\end{array}$ & $\begin{array}{l}\text { To study the effect of } \\
\text { gravity }\end{array}$ & $\begin{array}{l}\text { Eulerian and Lagrangian compared } \\
\text { and showed an alternative for two } \\
\text { phase model }\end{array}$ \\
\hline
\end{tabular}


Table (2.8-1). Continued (2)

\begin{tabular}{|c|c|c|c|c|}
\hline References & CFD Code & Approach Model & Objective & Findings/Comments \\
\hline Kee and Tan., 2002 & Fluent & MRF and $k-\varepsilon$ Eulerian & $\begin{array}{l}\text { To find out } N_{j s} \text { and } \\
\text { cloud height } \\
\text { numerically }\end{array}$ & $\begin{array}{l}N_{j s} \text { underestimated by comparing to } \\
\text { available empirical model }\end{array}$ \\
\hline Derksen., 2003 & & $\begin{array}{l}\text { Black box and } k-\varepsilon \\
\text { Lagrangian }\end{array}$ & $\begin{array}{l}\text { To study the effect of } \\
\text { collision and all other } \\
\text { forces }\end{array}$ & Good agreement qualitatively \\
\hline Wang et al., 2003 & Simple & $\begin{array}{l}\text { Black box and } k-\varepsilon \\
\text { Eulerian }\end{array}$ & $\begin{array}{l}\text { Concentration profile } \\
\text { (axially and radially) }\end{array}$ & Good agreement with $20 \%$ solid w/w \\
\hline $\begin{array}{l}\text { Sommerfeld and } \\
\text { Decker, } \\
2004\end{array}$ & $\begin{array}{l}\text { CFX, DSN, } \\
\text { LES }\end{array}$ & $\begin{array}{l}\text { MRF and } k-\varepsilon \text { RAN } \\
\text { Lagrangian }\end{array}$ & $\begin{array}{l}\text { To study the } \\
\text { concentration and } \\
\text { velocity profile }\end{array}$ & $\begin{array}{l}\text { Due to high computational time } \\
\text { DNS failed for Re }>8000, \text { LES good } \\
\text { agreement up to } 5 \% \mathrm{w} / \mathrm{w}\end{array}$ \\
\hline Micale et al., 2004 & CFX4.4 & SM and $k-\varepsilon$ Eulerian & $\begin{array}{l}\text { To find } N_{j s} \text { and axial } \\
\text { velocity profile }\end{array}$ & $\begin{array}{l}\text { Good agreement from } 1 \text { to } 10 \% \mathrm{w} / \mathrm{w} \\
\text { with literature }\end{array}$ \\
\hline Hao., 2005 & $\begin{array}{l}\text { Fluent 6.0, } \\
\text { SIMPELEC }\end{array}$ & $\begin{array}{l}\text { Black box and } k-\varepsilon \\
\text { Eulerian }\end{array}$ & $\begin{array}{l}\text { Velocity profile and } \\
\text { concentration profile, } \\
\text { effect of density and } \\
\text { particle size }\end{array}$ & $\begin{array}{l}\text { Cloud height good agreement, no big } \\
\text { difference in mixture size, } \\
\text { segregation is increased by } \\
\text { increasing density }\end{array}$ \\
\hline Spilda et al., 2005 & Fluent6.2 & MRF and $k-\varepsilon$ Eulerian & $\begin{array}{l}\text { To study the } \\
\text { concentration profile } \\
\text { at } N_{j s}\end{array}$ & $\begin{array}{l}\text { Particle up to 5\%w/w, Higher values } \\
\text { of CD recommended, no agreement } \\
\text { between e dissipation, Radial } \\
\text { concentration profile is more } \\
\text { gradient than experimental }\end{array}$ \\
\hline
\end{tabular}


Table (2.8-1). Continued (3)

\begin{tabular}{|c|c|c|c|c|}
\hline References & CFD Code & Approach Model & Objective & Findings/Comments \\
\hline $\begin{array}{l}\text { Ochieng and Lewis., } \\
2006\end{array}$ & CFX5.6/5.7 & $\begin{array}{l}\text { MRF initially and then } \\
\text { SM Eulerian }\end{array}$ & $\begin{array}{l}\text { Velocity and } \\
\text { concentration profile } \\
\text { with } N_{j s} \text {, clearance, } \\
\text { solid loading up to } \\
20 \% \text { particle size } \\
\text { effect }\end{array}$ & $\begin{array}{l}0.15 \mathrm{~T} \text { clearance adopted as the best } \\
\text { option, mesh more than } 300 \text { microns } \\
\text { has more deviation regarding the } \\
\text { experimental than empirical, cloud } \\
\text { height measurement failed both in } \\
\text { exp. and CFD for less than } 2.5 \% \text {, } \\
\text { visual method advised, } N_{j s} \text { has } 50 \% \\
\text { error for less than } 6 \% \mathrm{w} / \mathrm{w} \text { loading, } \\
\text { best for developing empirical if low } \\
\text { concentration }<6 \% \text { and } d_{P}<150 \\
\text { microns }\end{array}$ \\
\hline Ricard et al., 2005 & $\begin{array}{l}\text { Fluent } 6 \\
\text { SIMPELEC- } \\
\text { QUICK }\end{array}$ & MRF and $k-\varepsilon$ Eulerian & $\begin{array}{l}\text { To find axial } \\
\text { concentration profile } \\
\text { at } N_{j s} \text { and above }\end{array}$ & $\begin{array}{l}\text { Good agreement from } 1 \text { to } 10 \% \mathrm{w} / \mathrm{w} \\
\text { with literature and ERT. CFD } \\
\text { underestimated the concentration up } \\
\text { to } 20 \%\end{array}$ \\
\hline $\begin{array}{l}\text { Zhang and Ahmadi., } \\
2005\end{array}$ & & Lagrangian & $\begin{array}{l}\text { To study the } \\
\text { dispersion of different } \\
\text { phases }\end{array}$ & $\begin{array}{l}\text { Good agreement with } \\
\text { experiment/most of the interaction } \\
\text { between the phases are included }\end{array}$ \\
\hline Wang et al., 2006 & SIMPLE & $\begin{array}{l}\text { Black box and } k-\varepsilon \\
\text { Eulerian }\end{array}$ & $\begin{array}{l}\text { Velocity profile and } \\
\text { concentration profile, } \\
\text { for all three phases at } \\
\text { Black box and } N_{j s} \text {, } \\
\text { and above }\end{array}$ & $\begin{array}{l}\text { Good agreement/further } \\
\text { improvement need for CFD model }\end{array}$ \\
\hline
\end{tabular}


Table (2.8-1). Continued (4)

\begin{tabular}{|c|c|c|c|c|}
\hline References & CFD Code & Approach Model & Objective & Findings/Comments \\
\hline Khopkar et al., 2006 & Fluent6.2 & MRF and $k-\varepsilon$ Eulerian & $\begin{array}{l}\text { To study } \\
\text { concentration profile } \\
\text { at } N_{j s} \text { and effect of } \\
\text { particle size, drag } \\
\text { force }\end{array}$ & $\begin{array}{l}\text { Drag force coefficient should be } \\
\text { reduced by } 10 \text { times so that CFD is } \\
\text { applicable to predict objectives for } \\
d_{P} \text { less than } 655 \mu \mathrm{m} \text { and } 16 \% \mathrm{w} / \mathrm{w}\end{array}$ \\
\hline Tyagi et al., 2007 & & $\begin{array}{l}\text { Immersed boundary } \\
\text { method and LES } \\
\text { Lagrangian }\end{array}$ & $\begin{array}{l}\text { To study the best } \\
\text { velocity profile within } \\
\text { the tank }\end{array}$ & $\begin{array}{l}\text { Reasonable agreement } \\
\text { achieved/computationally very } \\
\text { expensive }\end{array}$ \\
\hline Fradette et al., 2007 & SIMM & $k-\varepsilon$ Eulerian & $\begin{array}{l}\text { Velocity profile and } \\
\text { concentration profile, } \\
\text { Clearance, particle } \\
\text { size }\end{array}$ & $\begin{array}{l}\text { The model is capable of predicting } \\
\text { suspension behaviour of particle }\end{array}$ \\
\hline Prat \& Ducoste., 2007 & $\begin{array}{l}\text { QMOM } \\
\text { Simple }\end{array}$ & $\begin{array}{l}\text { MRF and } k-\varepsilon \\
\text { Lagrangian and Eulerian }\end{array}$ & $\begin{array}{l}\text { To study the } \\
\text { concentration profile } \\
\text { at } N_{j s} \text { and effect of } \\
\text { particle size, Impeller }\end{array}$ & $\begin{array}{l}\text { Eulerian prediction is better and } \\
\text { more stable than Lagrangian }\end{array}$ \\
\hline Kasat et al., 2008 & Fluent 6.2 & MRF and $k-\varepsilon$ Eulerian & $\begin{array}{l}\text { To study the liquid } \\
\text { phase mixing with } \\
\text { different stages of } \\
\text { solid suspension }\end{array}$ & $\begin{array}{l}\text { Good agreement with experiment, } \\
\text { CFD model useful for large scale } \\
\text { stirred reactors. }\end{array}$ \\
\hline Ochieng et al., 2009 & SIMPLE & MRF and $k-\varepsilon$ Eulerian & $\begin{array}{l}\text { CFD simulation of } \\
\text { solid-liquid mixing in } \\
\text { stirred tank }\end{array}$ & $\begin{array}{l}\text { Good agreement with experiment, } \\
\text { CFD can provide detail data for the } \\
\text { system scale up. }\end{array}$ \\
\hline
\end{tabular}


Table (2.8-1). Continued (5)

\begin{tabular}{|l|l|l|l|l|}
\hline References & CFD Code & Approach Model & Objective & Findings/Comments \\
\hline Hosseini et al., 2010b & Fluent 6.3 & MRF and $k-\varepsilon$ Eulerian & $\begin{array}{l}\text { To study the solid } \\
\text { particle concentration } \\
\text { profile at } N_{j s} \text { and } \\
\text { effect of stirrer type, } \\
\text { stirrer speed, particle } \\
\text { size, stirrer clearance } \\
\text { homogeneity held good with } \\
\text { experimental results }\end{array}$ & $\begin{array}{l}\text { The CFD results for the extent of } \\
\text { Gohel et al., 2012 }\end{array}$ \\
\hline Fluent 13.0 & MRF and $k-\varepsilon$ Eulerian & $\begin{array}{l}\text { To study the effect of } \\
\text { turbulent dispersion } \\
\text { and drag models on } \\
\text { cloud height }\end{array}$ & $\begin{array}{l}\text { Good agreement with experiment/ } \\
\text { at over prediction of cloud height } \\
\text { assembly for single impeller }\end{array}$ \\
\hline $\begin{array}{l}\text { Sardeshpande and } \\
\text { Ranade, 2012 }\end{array}$ & MRF and $k-\varepsilon$ Eulerian & $\begin{array}{l}\text { To study hysteresis in } \\
\text { clouding height }\end{array}$ & $\begin{array}{l}\text { The model is able to explain } \\
\text { hysteresis and dynamic settling in } \\
\text { solid suspensions }\end{array}$ \\
\hline Chen and Xiao, 2013 & CFX5 & MRF and $k-\varepsilon$ Eulerian & $\begin{array}{l}\text { To study solid } \\
\text { distribution using } \\
\text { three side-entering } \\
\text { impellers }\end{array}$ & $\begin{array}{l}\text { Good agreement with experimental } \\
\text { results }\end{array}$ \\
\hline
\end{tabular}




\subsection{Research Objectives}

After a thorough literature review, it is imperative to mention that no comprehensive research work has been reported regarding the characterization of the local solid concentrations in a slurry reactor furnished by a Maxblend impeller. In fact, no study has been done to assess the local mixing quality for liquid-solid mixing operation equipped with a Maxblend impeller through CFD modeling. An effort has been made in this research work to examine the performance of the Maxblend impeller for the solid suspension in a slurry reactor using the ERT and CFD techniques. The key research objectives for this study are as follows:

- To quantify the particle concentration and distribution in a slurry reactor equipped through just suspended agitation speed, clouding height and homogeneity approaches

- To examine the impact of the agitation speed on the local mixing quality for a Maxblend impeller.

- To analyze the effect of baffles for the Maxblend impeller on the suspension of the solid particles.

- To explore the influence of physical characteristics of the solid particles such as particle specific gravity and average particle size on the degree of solid suspension.

- To study the impact of solid particle loading on the mixing quality in a slurry reactor.

- To analyze the effect of the stirrer off-bottom clearance on the solid-liquid mixing quality for the Maxblend impeller.

- To understand the effect of axial and radial particle concentration profiles on the distribution of solid particles in slurries for the Maxblend impeller.

- To assess the performance and effectiveness of the Maxblend impeller for solid-liquid mixing operations by comparing the efficiency of the Maxblend impeller to the efficiencies of the A200 (an axial-flow impeller) and Rushton (a radial-flow impeller). 


\section{Chapter 3}

\section{EXPERIMENTAL SETUP AND METHODOLOGY}

This chapter includes experimental setup, particle size analysis, ERT, homogeneity calculation, and tests conditions.

\subsection{Experimental Setup and Procedure}

In this investigation, all the experimental tests were carried out in a transparent cylindrical agitated tank of $600 \mathrm{~mm}$ height $(H)$ and $400 \mathrm{~mm}$ diameter $(T)$ as depicted in Figure (3.1-1). Four baffles with the width of $33 \mathrm{~mm}$ and clearance of $6 \mathrm{~mm}$ to the vessel wall were installed at equal spacing. All the baffles were removed while taking measurements for unbaffled conditions. Three types of impellers namely the Maxblend, A200 (axial flow) and Rushton (radial flow) were utilized in this work. The diameter $(D)$ of each of the impellers was $250 \mathrm{~mm}$. Four tomographic planes with the spacing of $85 \mathrm{~mm}$ between them were located around the periphery of the mixing vessel for ERT measurements. The top plane was numbered P1 and the subsequent lower planes were numbered P2, P3, and P4, respectively, as shown in Figure (3.1-1). Each sensor plane comprised of 16 sensing electrodes made of the stainless steel. These electrodes were fixed at the equal spacing at the boundary of the mixing tank. The dimensions of the electrodes were $20 \mathrm{~mm}$ height, $30 \mathrm{~mm}$ width, and $1 \mathrm{~mm}$ thickness, respectively. The co-axial cables were utilized to connect the electrodes to the tomography machine (ITS p2+, UK), which was connected to the host computer. The reference electrode, which acts as a ground electrode, was positioned between planes two and three. This setup was equipped with a variable frequency drive to control the impeller speed and a torque meter to measure the impeller torque. The height of liquid $(h)$ was kept at $475 \mathrm{~mm}$ with corresponding volume of $0.0597 \mathrm{~m}^{3}$ in all tests. Due diligence was given while adding solid particles and removing water to maintain the specified liquid level. All the tests were performed at room temperature. To maintain the substantial conductivity difference between the conductive liquid and non-conductive particles phase, small amount of $\mathrm{NaCl}$ was added to the system. A 
conductivity meter was utilized to measure the conductivity of the tap water. The conductivity of tap water was found to be $0.323 \mathrm{mS} / \mathrm{cm}$ before addition of the salt.

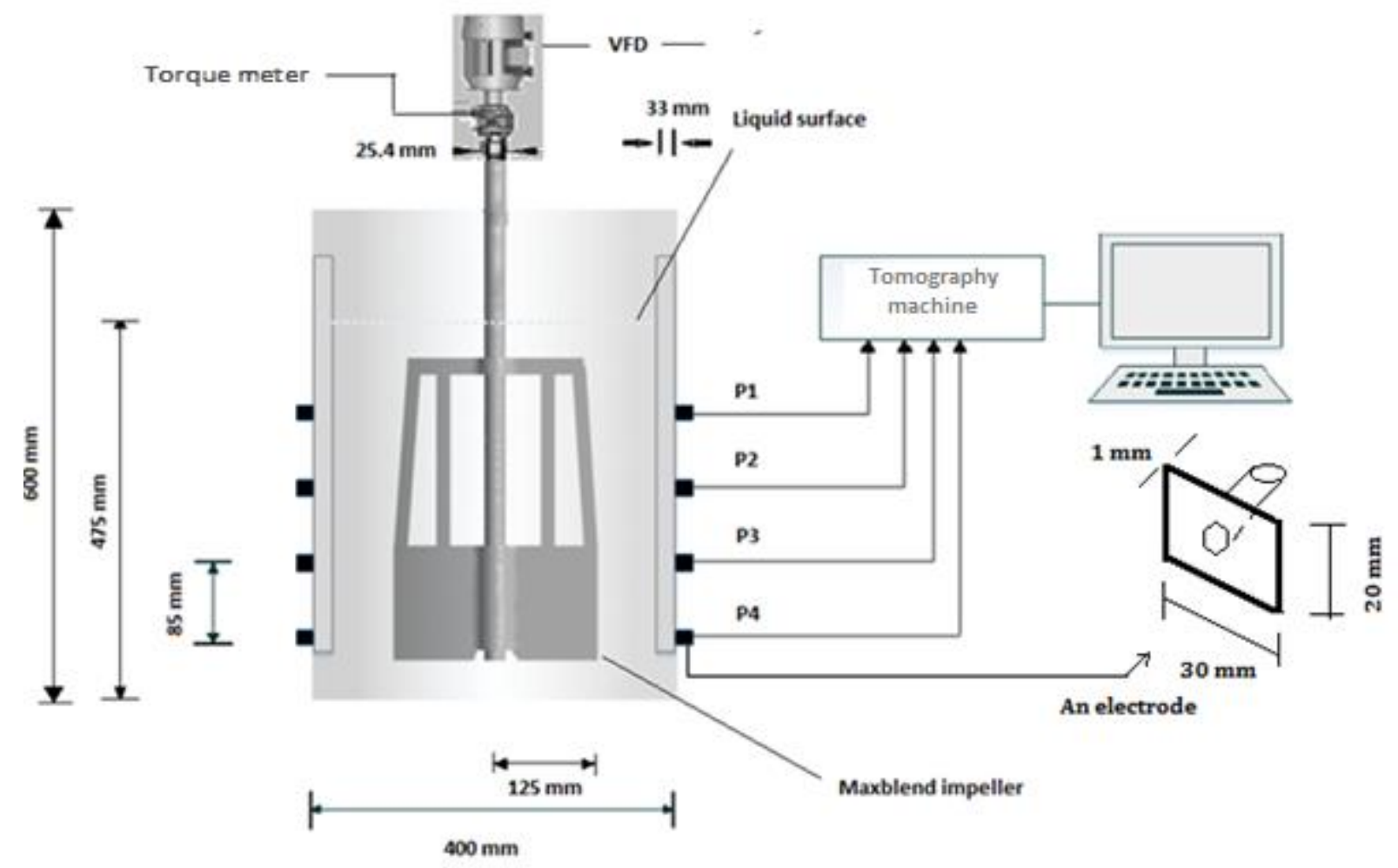

Figure (3.1-1). Experimental setup for this study.

\subsection{Particle Size Analysis}

Different sizes of Ballotini impact glass beads (Potters Industries Inc.) were used as the solid particle phase and tap water as the continuous (liquid) medium for this study. Table (3.1-1) provides the specifications of the particles (glass beads). 
Table (3.2-1). Specifications of glass beads

\begin{tabular}{|c|c|c|c|c|c|}
\hline Potters designation & Shape & $\begin{array}{l}\text { Maximum } \\
\text { size (Micron) }\end{array}$ & $\begin{array}{c}\text { Minimum size } \\
\text { (Micron) }\end{array}$ & $\begin{array}{c}\text { Density } \\
\left(\mathrm{Kg} / \mathrm{m}^{3}\right)\end{array}$ & Packing factor \\
\hline 3 & Spherical & 850 & 600 & 2500 & 0.60 \\
\hline 4 & Spherical & 600 & 425 & 2500 & 0.60 \\
\hline 7 & Spherical & 250 & 180 & 2500 & 0.65 \\
\hline
\end{tabular}

To assess the average particle size (diameter) and distribution, the laser diffraction particle size analyzer (Microtrac S3500, USA) was used. Average particle size $\left(d_{p}\right)$ of glass beads were found to be $209 \mu \mathrm{m}, 537 \mu \mathrm{m}$ and $752 \mu \mathrm{m}$, respectively. The size distributions of glass beads are illustrated in Figure (3.2-1).

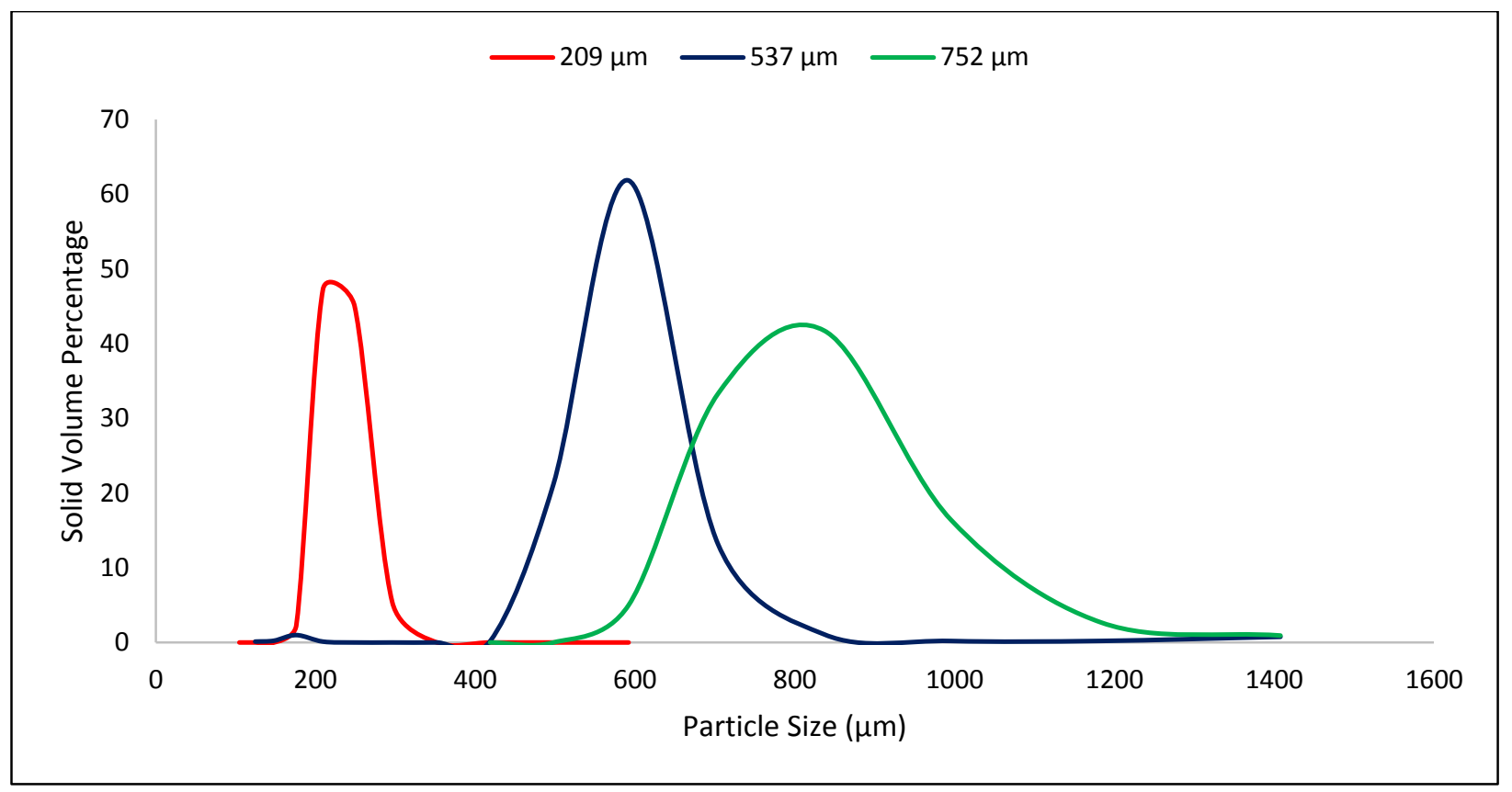

Figure (3.2-1). Glass beads particle size distributions. 


\subsection{Electrical Resistance Tomography (ERT)}

ERT is a simple, robust, and non-invasive/non-intrusive technique, which can be used in solid-liquid mixing operations to visualise the flow inside the mixing tank. In ERT, the conductivity distribution in the process tank is obtained by applying the current and simultaneous measurement of voltages with the help of sensing electrodes. A suitable and reliable algorithm is used to obtain the cross-sectional images also called as tomograms. An ERT system consists of three major components: (a) the sensing device (electrodes), (b) the data acquisition system (DAS), and (c) the reconstruction of image by using the host computer. It should be noted that the continuous phase must be conductive.

\subsubsection{Electrode Sensors}

Multiple electrodes are fixed on the periphery of the mixing tank at equal intervals to make electrical contact with the fluid. However, the sensing electrodes do not interfere or disturb the flow pattern inside the tank. Extreme care and caution should be given in designing the electrodes to obtain reliable and accurate conductivity values in the area of concern. The electrodes are connected to the tomography machine through the coaxial cables to prevent and reduce interference and noise coming from the surrounding environment (Dickin and Wang, 1996). The selection of material for electrodes greatly depend on processing system, cost effectiveness, installation method, conductivity values and inhibition to corrosion (Williams and Beck, 1995). To avoid problems arising from contact impedance, electrodes are made up of silver, platinum, stainless steel, silver palladium alloy or brass (Tapp and Williams, 2000). Factors, which need to be taken into considerations while determining the electrode dimensions, are: (a) tank diameter, (b) conductivity range, (c) imaging speed, and (d) velocity of process materials. A reference electrode is fixed away from the measuring electrodes to act as a ground electrode. It has been reported that the ratio of the electrode diameter to the inter-electrode gap should be equal to 0.4 to achieve the better results.

\subsubsection{Data Acquisition System (DAS)}

Data acquisition system (DAS) acquires the quantitative conductivity data through sensing electrodes and allows a suitable algorithm to reconstruct the images. Five major strategies can be ascribed to DAS to measure voltages: (a) opposite (Viergever and Todd-Pokropek, 1988), (b) 
diagonal (Hua et al., 1993), (c) adjacent (Pakzad et al., 2008; Hosseini et al., 2010a; Tahvildarian et al., 2011), (d) linear, and (e) conducting boundary. Due to less complications associated with adjacent strategy, it was used for voltage measurements in this study.

In case of the adjacent protocal, an injection current is provided through a pair of neighbouring electodes and difference in voltages is measured from the remaining neighbouring electrode pairs. This mode of current interjection and voltage measurents is repeated for all electrode pairs to acquire all the indepenedent measurements possible. By using the following correlation, number of independent ERT voltage measurements can be obtained:

$$
M_{e}=\frac{n_{s e}\left(n_{s e}-3\right)}{2}
$$

where $M_{e}$ and $n_{s e}$ are the number of independent voltage measurements and the number of electrode sensors, respectively. By this method, 16 electrode sensors give 104 independent voltage values. It is imperative to have good data collection strategy (the ability to identify two different conductivity values) to avoid misleading image reconstruction. If on-line processing of image is not required, the fast adjacent strategy can be appllicable to collect data quickly.

\subsubsection{Image Reconstruction System (Host Computer)}

Data collected by DAS are then conveyed to the host computer to obtain the reconstructed images using an appropriate and reliable algorithm. Two major types of algorithms reported in the literature for the reconstruction of images are: (a) iterative algorithm (sensitivity conjugate gradient method) (Wang, 2002) and (b) non-iterative algorithm also referred to as the linear back

projection (Williams and Beck, 1995). In the linear back projection algorithm, which was employed in this study, forward solving method is employed to calculate the potential difference. In this algorithm, the tomogram is obtained by the relative difference in the conductivty values between a reference and test sets. The reference was taken with the tap water using the multiple reference frames and saved for the future references in this investigation. The reconstructed conductivity tomogram consists of a square reconstruction grid of a size of 400 pixels [20 $\times 20]$. 
Only 316 pixels out of these 400 pixels are used to construct the circular tomograms because the remaining 84 pixels do not lie inside the tank circumference.

Some of the shortcomings of ERT are (Dickin and Wang, 1996): (a) delay (time lag) in measurement of conductivities between top and bottom planes, (b) low resolution ( $5-10 \%$ of the tank diameter), and (c) optical aberrations in conductivity tomograms arising from the end effects.

\subsubsection{Parameters for ERT Measurements}

The parameters for ERT measuremets are listed in Table (3.3-1).

Table (3.3-1). Parameters for ERT measurements

\begin{tabular}{cc}
\hline Specifications & Value \\
\hline Injection current $[\mathrm{mA}]$ & 15 \\
Frequency [Hz] & 9600 \\
Sampling time intervals [ms] & 20 \\
Maximum number of frames & 250 \\
Frames per download & 250 \\
\hline
\end{tabular}

\subsection{Power and Homogeneity Calculation}

The power consumption $(P)$ was calculated from the agiatation speed $(N)$ and impeller torque $(M)$ using the following equation:

$$
P=2 \pi N M
$$

The solid particles concentration can be determined from the conductivity values retrieved from ERT measurements by using Maxwell's equation (Hosseini et al., 2010a): 


$$
X_{v}=\frac{2 \sigma_{l}+\sigma_{s}-2 \sigma_{m c}-\left[\frac{2 \sigma_{m c} 2 \sigma_{s}}{2 \sigma_{l}}\right]}{\sigma_{m c}-\left(\frac{\sigma_{s}}{\sigma_{l}}\right) \sigma_{m c}+2\left(\sigma_{l}-\sigma_{s}\right)}
$$

where $X_{v}, \sigma_{s}, \sigma_{l}$ and $\sigma_{m c}$ are solid volume fraction, particle phase conductivity, liquid phase conductivity, and conductivity of the slurry respectively. Hosseini et al. (2010a) used glass beads as dispersed phase and considered conductivity of this phase to be zero $\left(\sigma_{s}=0\right)$ to obtain a simplified form of Equation (3.4-2) as follows:

$$
X_{v}=\frac{\sigma_{l}-\sigma_{m c}}{\sigma_{l}+0.5\left(\sigma_{m c}\right)}
$$

Homogeneity can be calculated by (Hosseini et al., 2010a):

$$
\text { Homogeneity }=1-\sqrt{\frac{\sum_{1}^{q}\left(X_{v}-\overline{X_{v}}\right)^{2}}{q}}
$$

where $q$ is the number of planes and $\overline{X_{v}}$ is the average solid volume fraction. The homogeneity was calculated in this study using Equation (3.4-4).

\subsection{Tests Conditions}

In this work, the effects of various significant parameters such as use of baffles, impeller speed, particle size, solid loading and impeller clearance were examined to assess the performance 
of the Maxblend impeller in terms of level of homogeneity and mixing index. The test conditions employed in this study are presented in Table (3.5-1).

Table (3.5-1). Experimental conditions

\begin{tabular}{ll}
\hline Variable & Range \\
\hline Geometrical configuration & Baffled, Unbaffled \\
\hline Impeller speed & $180-600 \mathrm{rpm}$ \\
\hline Impeller types & Maxblend, A200, Rushton \\
\hline Particle size & $209-752$ microns \\
\hline Solid concentration & $5-30 \mathrm{wt} \%$ \\
\hline Impeller clearance & $T / 8-T / 4$
\end{tabular}

\subsection{Error Analysis}

All the ERT tests were repeated three times under the same processing conditions for the reproducibility purpose and the standard deviation values of the conductivity $(\mathrm{mS} / \mathrm{cm})$ were noticed to be less than $1.4 \%$. In order to mitigate the random errors of the torque sensor caused by friction, mechanical play, and electronic fluctuations, torque measurements were recorded three times under the same operating conditions. The average of the three torque values were employed in this study. The maximum standard deviation in the torque measurement was found to be less than $0.5 \%$. 


\section{Chapter 4}

\section{CFD SIMULATION AND NUMERICAL MODELING}

In order to model the mixing of solid particles inside the stirred tank, ANSYS FLUENT (V15.07) was employed in this study. The geometry, mesh, and simulations were created employing ANSYS DesignModeler, ANSYS Meshing, and ANSYS FLUENT, respectively. This chapter comprises governing equations, grid generation and boundary conditions, grid independency, and convergence criteria for CFD modeling.

\subsection{Governing Equations}

Eulerian and Eulerian (E-E) multiphase fluid model was employed in this study for the formulation of the numerical model. Equations of continuity and momentum were applied for solid-liquid phase flow to obtain the resulting transport equations for phase $q$.

Continuity equation can be given by (Bird et al., 2002):

$$
\frac{\partial\left(a_{q} \rho_{q}\right)}{\partial t}+\nabla \cdot\left(a_{q} \rho_{q} \vec{u}_{q}\right)=0
$$

where $a, \vec{u}$, and $\rho$ are volume fraction, velocity vector, and density of fluid, respectively. Subscript $q$ denotes the phase $q$.

The momentum equation can be expressed as follows (Bird et al., 2002):

$$
\begin{aligned}
& \frac{\partial}{\partial t}\left(a_{q} \rho_{q} \vec{u}_{q}\right)+\nabla \cdot\left(a_{q} \rho_{q} \vec{u}_{q} \vec{u}_{q}\right)= \\
& -a_{q} \nabla p+\nabla \cdot\left(\overline{\overline{\tau_{q}}}\right)+a_{q} \rho_{q} \vec{g}+\left(\vec{F}_{D q}+\vec{F}_{B q}+\vec{F}_{L q}+\vec{F}_{v m q}\right)
\end{aligned}
$$


where $\overline{\overline{\tau_{q}}}$ represents the $q^{\text {th }}$ phase stress tensor and can be expressed as:

$$
\overline{\overline{\tau_{q}}}=a_{q}\left(\mu_{q}+\mu_{q}^{(t)}\right)\left(\nabla \vec{u}_{q}+\nabla \vec{u}_{q}^{T}\right)-\frac{2}{3} a_{q} \rho_{q} k_{q} \overline{\bar{I}}
$$

where $k_{q}, \mu_{q}^{(t)}$, and $\mu_{q}$ are turbulent kinetic energy, turbulent viscosity, and shear viscosity, respectively. $\vec{F}_{D q}$ represents the drag force. $\vec{F}_{v m q}, \vec{F}_{L q}$, and $\vec{F}_{B q}$ are added mass force, lift force, and buoyant force, respectively. $\overline{\bar{I}}$ is the unit tensor. Drag force can be given by:

$$
\vec{F}_{D}=\sum K_{l q}\left(\vec{u}_{l}-\vec{u}_{q}\right)
$$

where $K_{l q}$ represents coefficient of exchange between $q^{\text {th }}$ phase and the liquid phase. The combined effects of buoyant force and gravity force can be expressed as:

$$
\vec{F}_{B}+\vec{F}_{G}=\frac{\pi}{6} d_{p}^{3}\left(\rho_{p}-\rho_{l}\right) \vec{g}
$$

where $\rho_{l}, \rho_{p}, d_{p}$, and $\vec{g}$ are liquid density, particle density, particle diameter, and acceleration due to gravity, respectively. Added mass force can be expressed as (Zhang and Ahmadi, 2005):

$$
\vec{F}_{v m}=-\frac{\pi}{12} d_{p}^{3} \rho_{l} \frac{d}{d t}\left(\vec{u}_{p}-\vec{u}_{l}\right)
$$


where $\vec{u}_{l}$ and $\vec{u}_{p}$ are the liquid and particle velocities, respectively. $K_{l q}$ for dispersed (particle) phase can be denoted as $K_{p l}$ and expressed as:

$$
K_{p l}=\frac{3 a_{p} a_{l} \rho_{l}}{4 u_{t p} 2 d_{p}} C_{D} \frac{R e_{r}}{u_{t p}}\left|\vec{u}_{p}-\vec{u}_{l}\right|
$$

where the subscripts $p$ and $l$ represent the particle and liquid phase, respectively. $C_{D}, R e_{r}$, and $u_{t p}$ are drag coefficient, relative Reynolds number, and the particle terminal velocity, respectively. $R e_{r}$ can be expressed as:

$$
R e_{r}=\frac{\rho_{l} d_{p}\left|\vec{u}_{p}-\vec{u}_{l}\right|}{\mu_{l}}
$$

where $\mu_{l}$ is the viscosity of the liquid. Drag coefficient $\left(C_{D}\right)$ was calculated using the following equation (Gidaspow et al., 1992):

$$
\begin{aligned}
C_{D} & =\frac{24}{a_{l} R e_{r}}\left[1+0.15\left(a_{l} R e_{r}\right)^{0.687}\right] \quad \text { if } R e_{r} \leq 1000 \\
\text { and } \quad C_{D} & =0.44 \quad \text { if } R e_{r}>1000
\end{aligned}
$$

The lift force can be calculated as (Saffman, 1965; Derksen, 2003):

$$
\vec{F}_{L}=\frac{\pi}{4} d_{p}^{3} \frac{\rho_{l}}{2} C_{S}\left(\left(\vec{u}_{l}-\vec{u}_{p}\right) \times \omega\right)
$$


where $C_{S}=0.1524$ if $R e_{p} \geq 40 . \omega$ is the angular velocity. If $R e_{p} \leq 40, C_{S}$ can be calculated as (Saffman, 1965; Derksen, 2003):

$$
C_{S}=\frac{4.1126}{R e_{S R}^{0.5}}\left[\left(1.0-0.234\left(\frac{R e_{S R}}{R e_{p}}\right)^{0.5}\right) e^{-0.1 R e_{p}}+0.234\left(\frac{R e_{S R}}{R e_{p}}\right)^{0.5}\right]
$$

where $R e_{S R}$ and $R e_{p}$ are rotational Reynolds number and particle Reynolds number, respectively. $R e_{S R}$ can be calculated as:

$$
R e_{S R}=\frac{|\omega| d_{p}^{2}}{v}
$$

where $v$ represents the kinematic viscosity of the liquid medium. $R e_{p}$ can be calculated as:

$$
R e_{p}=\frac{\rho_{l} d_{p} u_{t p}}{\mu}
$$

In order to compute eddy viscosity $\left(\mu^{(t)}\right)$, the additional two transport equations were solved employing the $k-\varepsilon$ turbulence model (Launder and Spalding, 1972). The equation for kinetic energy of turbulence $(k)$ can be given by:

$$
\frac{\partial(\rho k)}{\partial t}+\frac{\partial}{\partial x_{i}}\left(\rho k \vec{u}_{i}\right)=\frac{\partial}{\partial x_{j}}\left(\left(\mu+\frac{\mu^{(t)}}{\sigma_{k}}\right) \frac{\partial k}{\partial x_{j}}\right)+G_{k}+G_{b}-\rho \varepsilon-Y_{m}
$$

The equation for dissipation rate of turbulence $(\varepsilon)$ is as follows: 


$$
\frac{\partial(\rho \varepsilon)}{\partial t}+\frac{\partial}{\partial x_{i}}\left(\rho k \vec{u}_{i}\right)=\frac{\partial}{\partial x_{j}}\left(\left(\mu+\frac{\mu^{(t)}}{\sigma_{\varepsilon}}\right) \frac{\partial k}{\partial x_{j}}\right)+C_{1 \varepsilon} \frac{\varepsilon}{k}\left(G_{k}+C_{3 \varepsilon} G_{b}\right)-C_{2 \varepsilon} \rho \frac{\varepsilon^{2}}{k}-Y_{m}
$$

where $G_{k}, G_{b}$, and $Y_{m}$ are turbulent kinetic energies developed due to velocity gradient, buoyancy effect, and fluctuating distension of eddies, respectively. $C_{1 \varepsilon}, C_{2 \varepsilon}, C_{3 \varepsilon}, \sigma_{k}$, and $\sigma_{\varepsilon}$ are empirical constants. The eddy viscosity $\left(\mu^{(t)}\right)$ can be expressed as:

$$
\mu^{(t)}=\rho C_{\mu} \frac{k^{2}}{\varepsilon}
$$

where $C_{\mu}$ is constant and $G_{k}$ can be expressed as:

$$
G_{k}=-\rho \overrightarrow{u_{\imath}^{\prime} u_{\jmath}^{\prime}} \frac{\partial u_{j}}{\partial x_{i}}
$$

In the above equation, $\overrightarrow{u_{\imath}^{\prime} u_{\jmath}^{\prime}}$ is referred to as the turbulent stresses. It is imperative to mention that the drag force and the forces interacting between the particle and fluid phase become dominant in comparison to the virtual mass force, the lift force, and the buoyant force if $\frac{\rho_{p}}{\rho_{l}}>2$.

\subsection{Geometry}

ANSYS DesignModeler was employed to create a stirred vessel with identical geometry to that one used in the experimental investigations. A cylindrical stirred vessel (transparent and flat bottomed) of $400 \mathrm{~mm}$ diameter $(T)$ and $600 \mathrm{~mm}$ height $(H)$, as mentioned in section (3.1), was used for carrying out all the simulations. The agitated vessel was equipped with four baffles (fixed at equal spacing) with the width of $33 \mathrm{~mm}$ and the clearance of $6 \mathrm{~mm}$ to the tank wall. The Maxblend impeller with a diameter of $250 \mathrm{~mm}$ was employed in this work. Glass beads of different sizes were utilized as the particle phase and the tap water as the liquid medium. The liquid height 
(h) was maintained at $47.5 \mathrm{~cm}$ in all the simulations. The rotation of the Maxblend impeller in the stirred tank was simulated using the sliding mesh (SM) technique (Tamburini et al., 2013; Kazemzadeh et al., 2016). Since the SM technique was employed for the simulation, a separate rotating fluid zone was defined around the Maxblend impeller.

\subsection{Grid Generation and Boundary Conditions}

Grid interfaces were defined to interchange the data. The unstructured tetrahedral grids were employed for discretization of the fluid flow domain in order to solve the equations of continuity and momentum. To obtain the precise and reliable flow information, very fine grid elements were created close to the walls. The growth rate of the mesh from the surface of the Maxblend impeller or the vessel wall was ensured by the mesh size function. No penetrations and no-slip boundary conditions were considered on the shaft, the bottom, and the vessel wall. Normal velocity on the fluid surface was considered to be zero. Modeling of the near wall zones was accomplished by using the standard wall function.

\subsection{Grid independency}

The grid independence test was carried out to obtain optimal number of grids. To achieve this goal, three grid systems were created: 671,059 cells, 1,340857 cells, and 2,801,357 cells as shown in Figure (4.4-1,2). The root mean square (RMS) deviations between the grid systems of $1,340,857$ and 2,801,357 cells in terms of the velocity magnitude and the solid volume fraction were less than $1.28 \%$. Hence, $1,340,857$ grids were used for simulating the fluid flow domain generated by the Maxblend impeller in this study. 


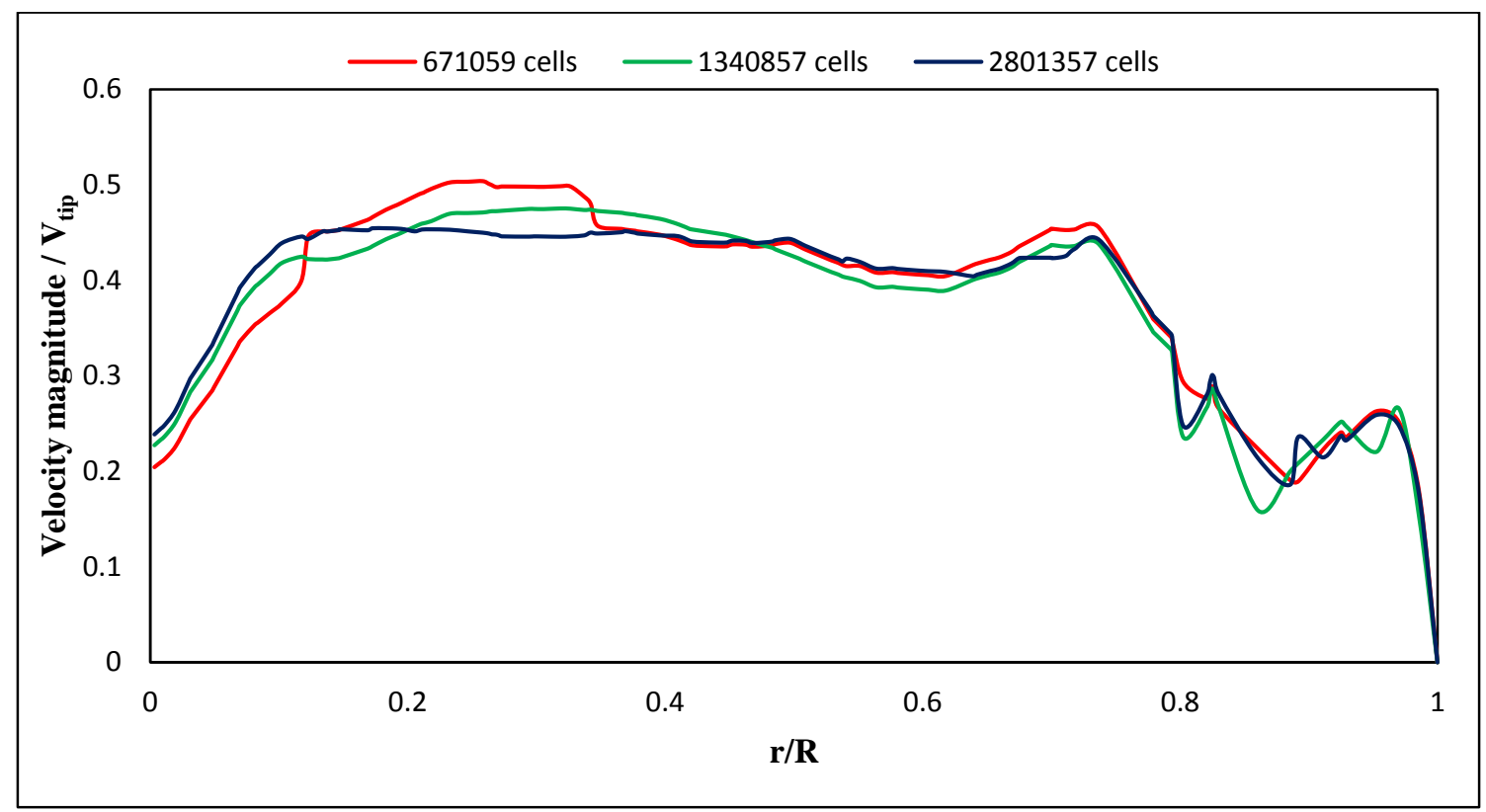

Figure (4.4-1). Grid independency: Axial velocity profiles at $1.5 \mathrm{~cm}$ below the Maxblend impeller $\left(N=480 \mathrm{rpm}, X=30 \mathrm{wt} \%, d_{p}=209 \mu \mathrm{m}, C=T / 8\right)$.

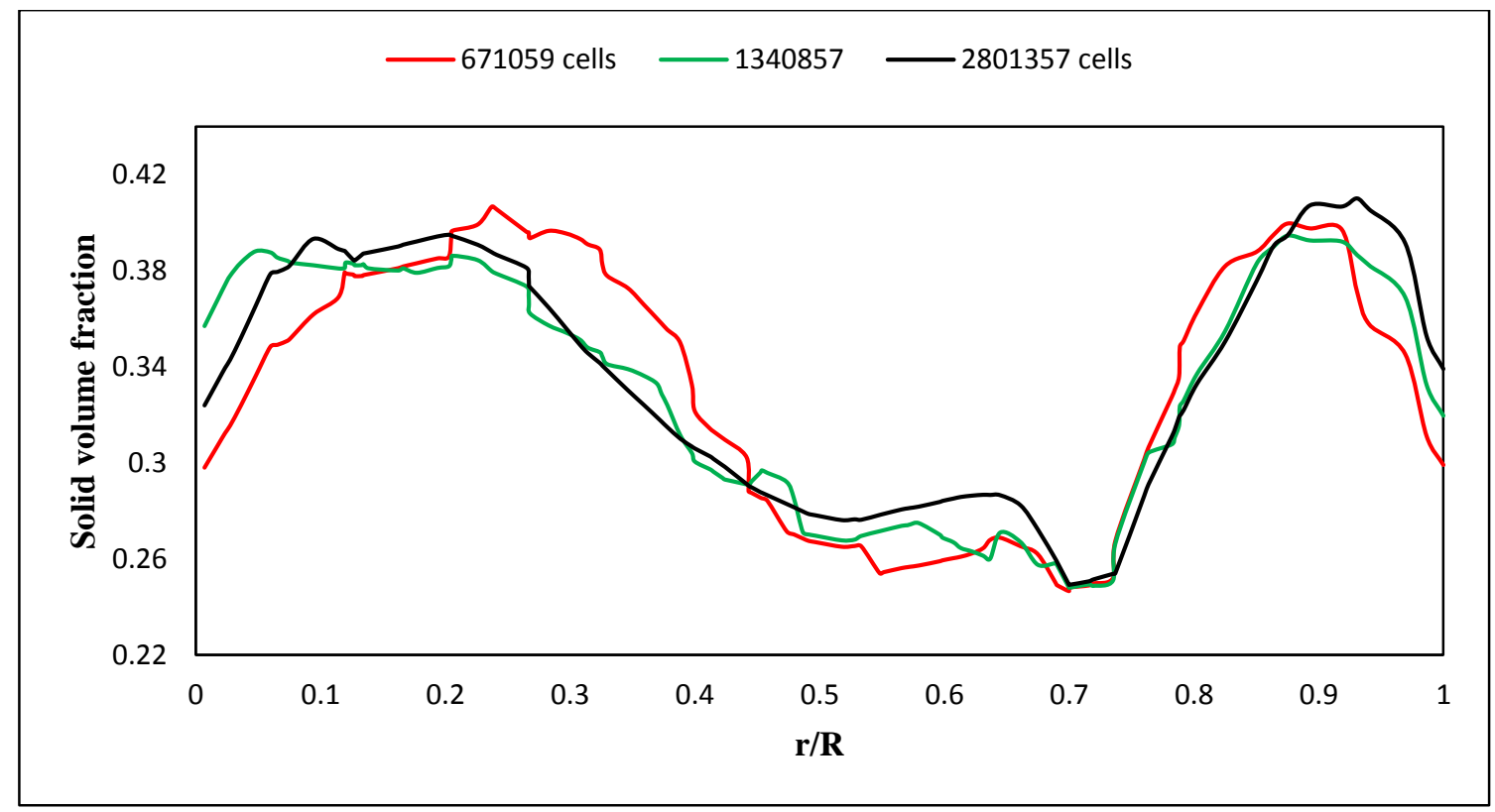

Figure (4.4-2). Grid independency: Solid concentration profiles at $1.5 \mathrm{~cm}$ below the Maxblend impeller $\left(N=480 \mathrm{rpm}, X=30 \mathrm{wt} \%, d_{p}=209 \mu \mathrm{m}, C=T / 8\right)$. 


\subsection{Convergence}

All the simulations were carried out at a time step of $0.001 \mathrm{~s}$. The convergence for the simulation was attained for all transport equations with the scaled residual values below $10^{-3}$ (Figure 4.5-1). The time required for computation of each simulation was about 60-72 h. HPCVL (High Performance Computing Virtual Laboratory) computing facilities were utilized to carry out all the simulations on $1.8 \mathrm{GHz}$ CPUs (12 dual core Sun Ultra-spark IV).

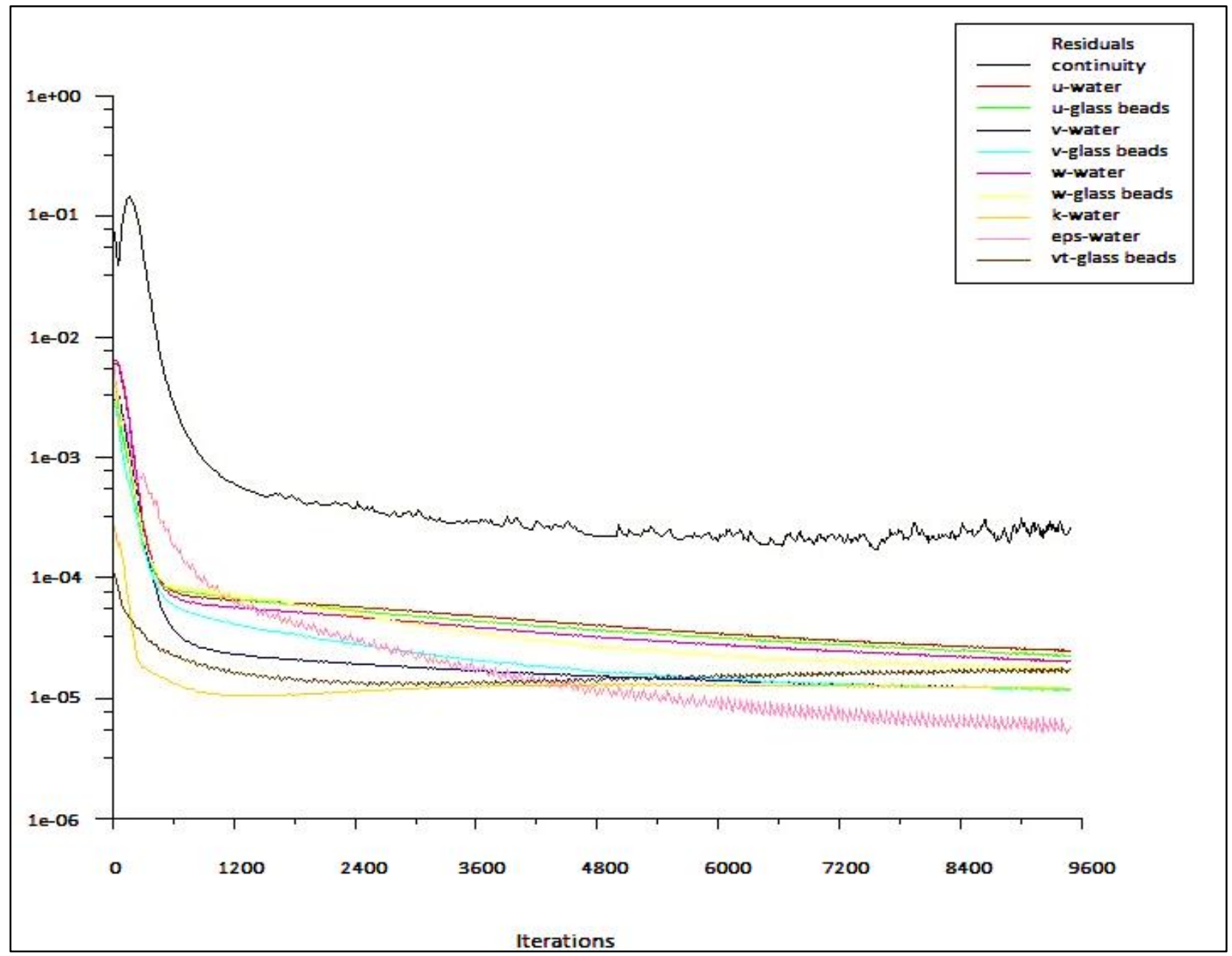

Figure (4.5-1). Scaled residuals for complete glass beads suspension. 


\subsection{Tests Conditions for CFD Simulations}

The test conditions for CFD simulations are presented in Table (4.6-1).

Table (4.6-1). Tests conditions

Variables $\quad$ Range

Geometrical configuration $\quad$ Baffled, Unbaffled

$\begin{array}{ll}\text { Impeller speed } & 180-600 \mathrm{rpm}\end{array}$

Impeller type $\quad$ Maxblend

$\begin{array}{ll}\text { Particle size } & 111-1,100 \text { microns }\end{array}$

$\begin{array}{ll}\text { Solid concentration } & 5-30 \mathrm{wt} \%\end{array}$

$\begin{array}{ll}\text { Impeller clearance } & T / 8\end{array}$

$\begin{array}{ll}\text { Specific gravity } & 1.5-6.0\end{array}$ 


\section{Chapter 5}

\section{RESULTS AND DISCUSSIONS}

In this work, the performance of the Maxblend impeller for the solid suspension in a slurry reactor was assessed using ERT and CFD techniques. In order to achieve this goal, the effects of various significant parameters such as use of baffles, impeller speed, particle size, solid loading, and impeller clearance were analyzed. In this chapter, all results and discussions are presented in the two separate sections.

\subsection{Using Tomograms to Assess the Local Solid Concentrations in a Slurry Reactor Equipped with a Maxblend Impeller}

The experimental setup mentioned in Chapter 3 was employed in this work. All the tests were repeated three times under the same processing conditions and the standard deviation values of the conductivity $(\mathrm{mS} / \mathrm{cm})$ were noticed to be less than $1.4 \%$. All the results are demonstrated and discussed for baffled conditions unless unbaffled conditions are mentioned.

Tomographic images (2D - tomograms) are depicted for solid loading of $20 \mathrm{wt} \%$ in Figure (5.1-1). The key concept of colors in these tomograms is for the interpretation of the conductivity, which is ultimately used for the assessment of the solid particles distribution in the mixing vessel. Since tap water (liquid phase) was conductive and the glass beads (particle phase) was nonconductive, the regions with blue color in the tomograms demonstrate the zones of higher solid loading. At the impeller speed $N=240 \mathrm{rpm}$, the blue areas in the plane $\mathrm{P} 4$ shows the accumulation of the particles at the base of the mixing vessel. The intensity of the blue area subsequently decreased from the bottom plane $(\mathrm{P} 4)$ to the top planes indicating that all the particles were not dispersed uniformly throughout the mixing vessel. As the agitation speed increased to $420 \mathrm{rpm}$, 
blue areas vanished indicating that the solid particles were uniformly distributed in the mixing tank.

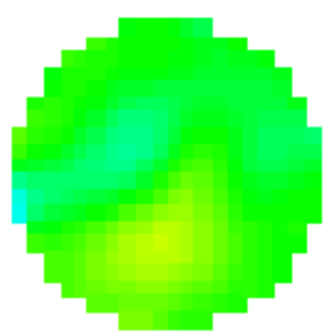

P1

$240 \mathrm{rpm}$

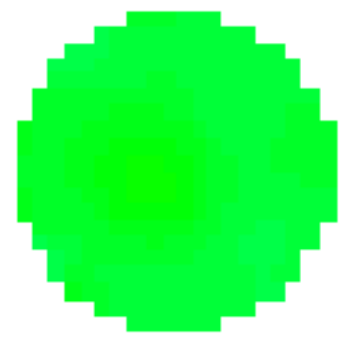

P1

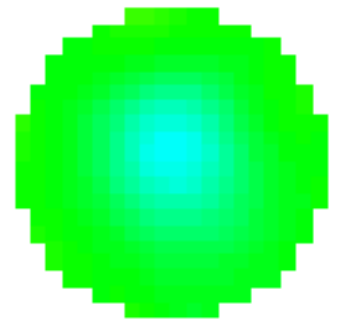

P2

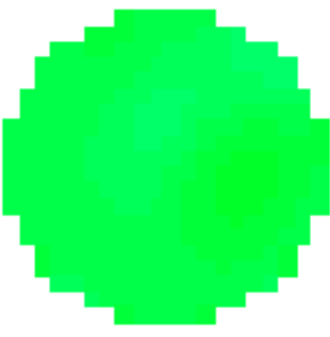

P2

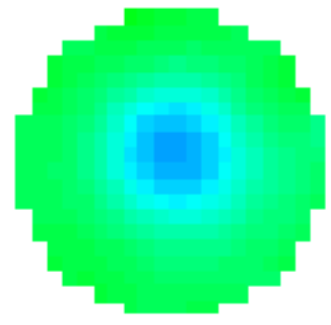

P3

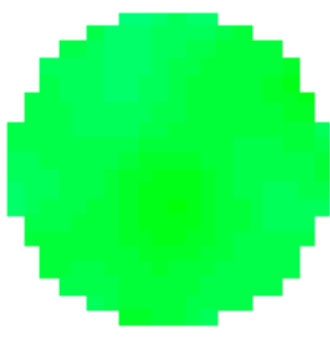

P3

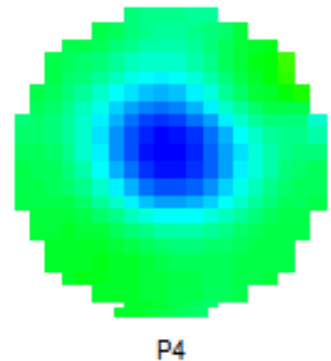

P4

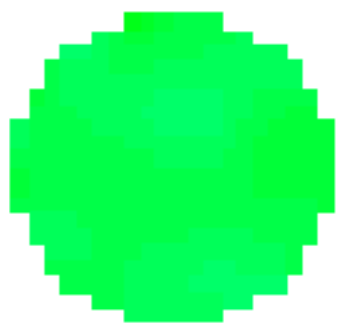

P4

$420 \mathrm{rpm}$

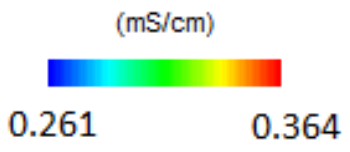

Figure (5.1-1). Conductivity tomograms acquired for the particle suspensions agitated at two stirrer speeds (Maxblend impeller, $C=T / 8, X=20 \mathrm{wt} \%$, and $d_{p}=752 \mu \mathrm{m}$ ).

Figure (5.1-2) shows the instantaneous dimensionless mean conductivity on four ERT sensor planes for the given conditions. The dimensionless mean conductivity values for tap water and solid particles were 1 and 0 , respectively. It can be seen that the particle concentration was 
almost nil in Plane P1. However, the solid concentration increased from Plane P2 to Plane P4. The fluctuating signals attained for P2-P4 was because of the movement of the solid particles in the lower planes during the mixing operations.

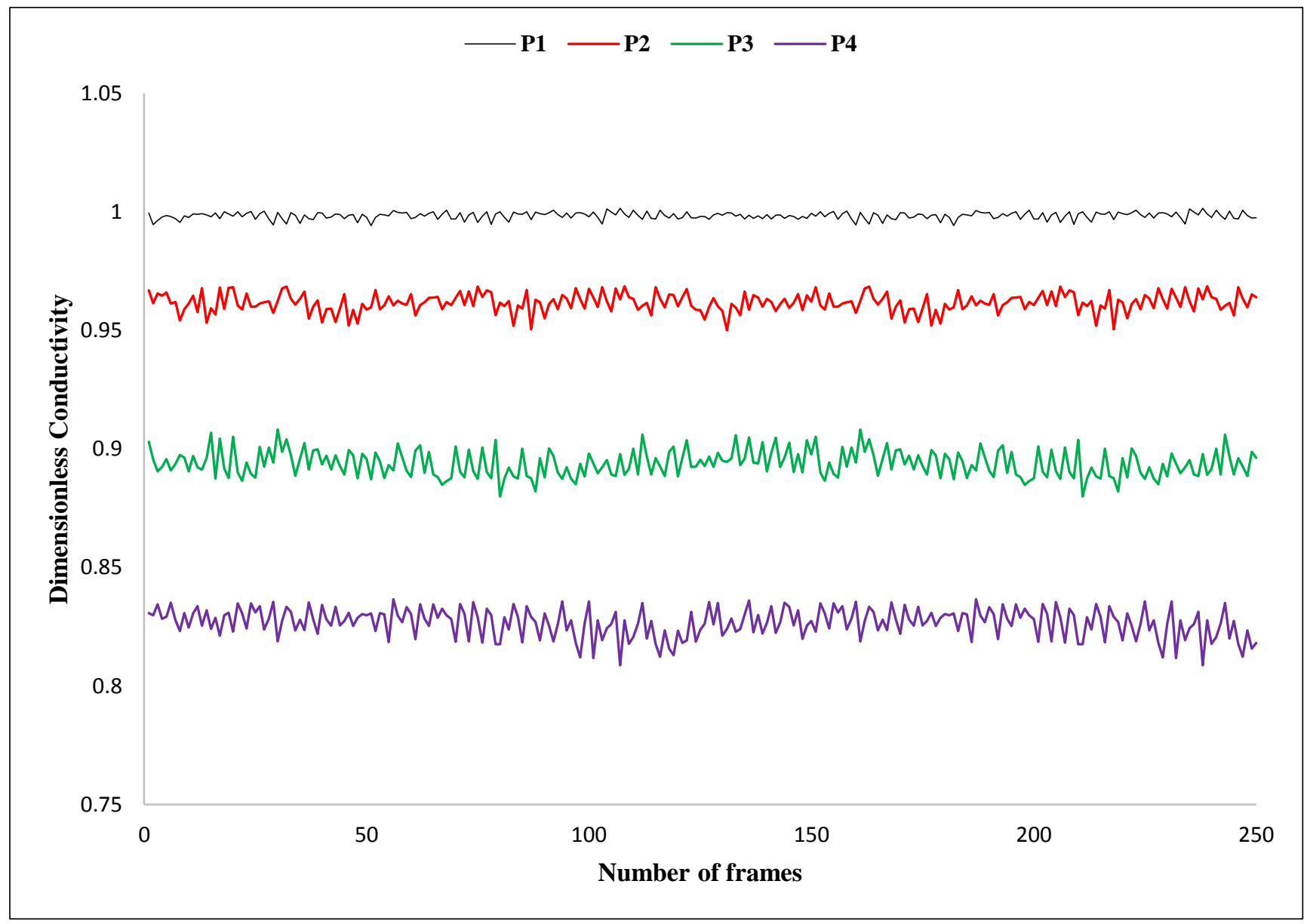

Figure (5.1-2). Dimensionless mean conductivity (Maxblend impeller, $C=T / 8, X=10 \%, d_{p}=209$ $\mu \mathrm{m}$, and $N=180 \mathrm{rpm}$ ).

To clearly assess the local solid distribution inside the vessel, the vertical sliced images and 3D tomographic images at the solid loading of $10 \mathrm{wt} \%$ are depicted in Figure (5.1-3). 


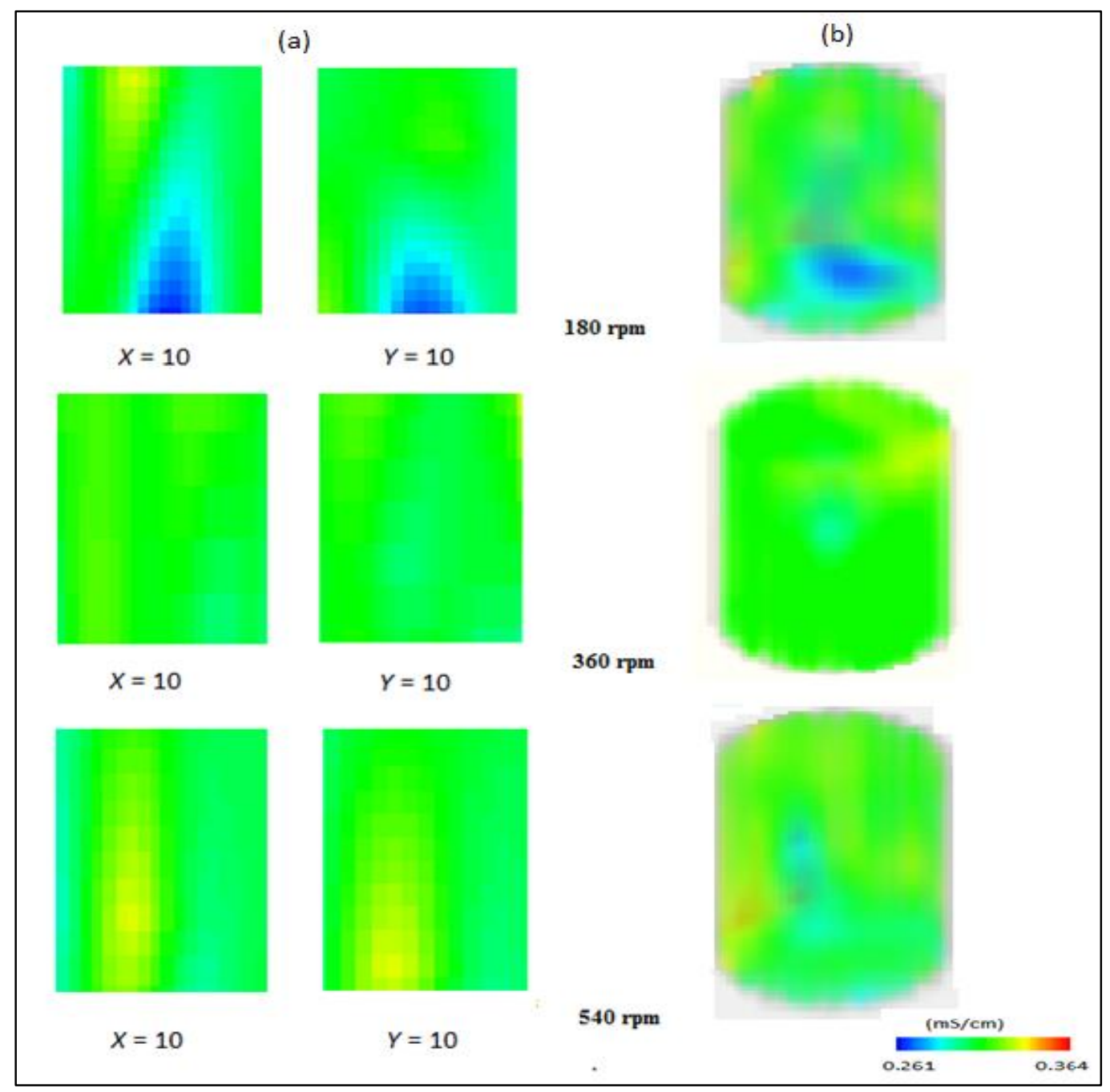

Figure (5.1-3). Solid particle distributions at three different agitation speeds: (a) vertical sliced images, and (b) 3D images (Maxblend impeller, $C=T / 8, X=10 \%$, and $d_{p}=537 \mu \mathrm{m}$ ).

These images show the accumulation of the solid particles at the base of the mixing tank at the agitation speed of $180 \mathrm{rpm}$. When the agitation speed increased to $360 \mathrm{rpm}$, a uniform distribution of the solid particles throughout the tank was achieved and thus the degree of homogeneity enhanced. Further raise in the agitation speed to $540 \mathrm{rpm}$ decreased the extent of homogeneity indicating that the solid particles were not distributed uniformly inside the mixing vessel. SlicerDicer (PIXOTEC, USA) software was utilized to develop the 3D image from the 2D tomographic images to precisely observe the particle distribution inside the mixing vessel (Figure 5.1-4). This figure confirms that the solid particles were not distributed uniformly at agitation speed of 300 rpm. 


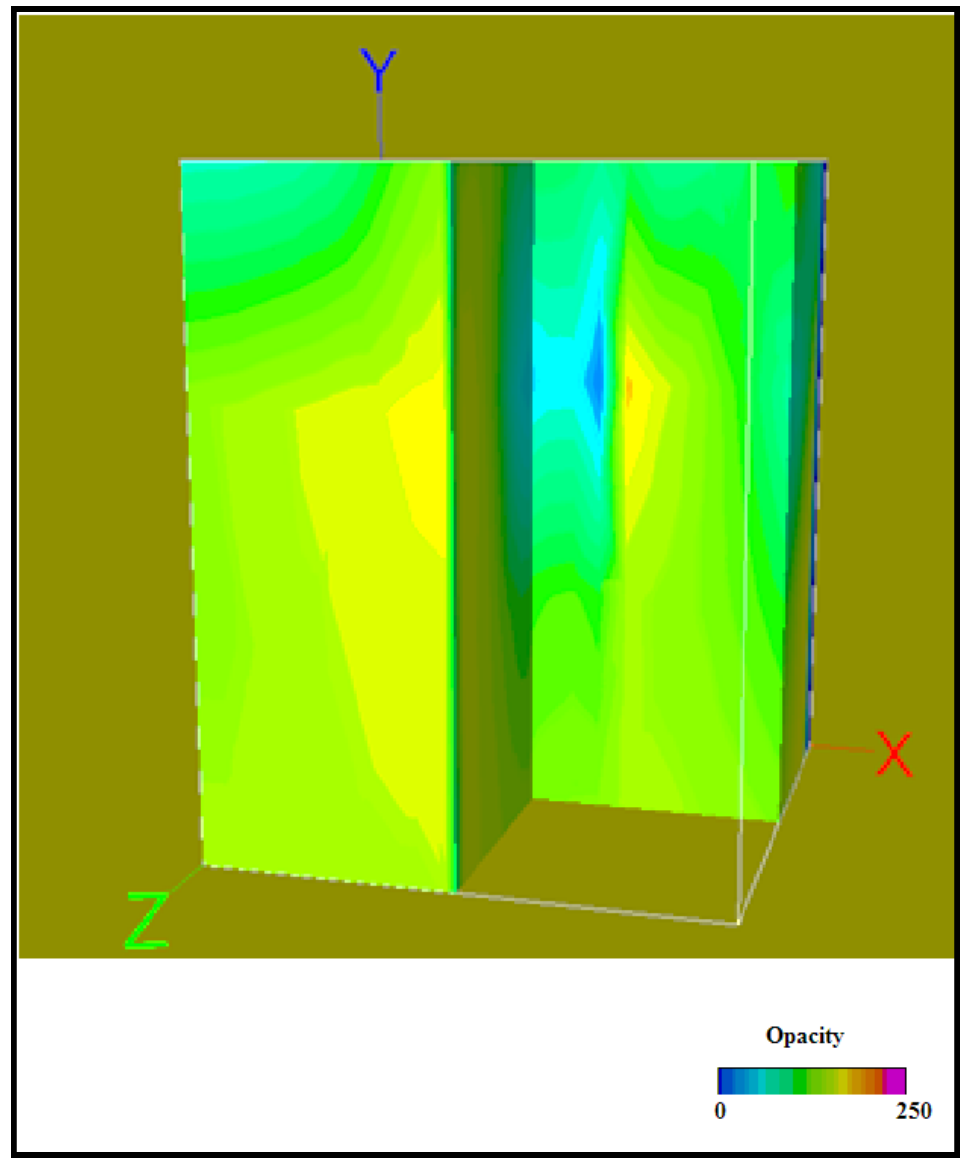

Figure (5.1-4). 3D image developed by slicer-dicer for Maxblend impeller $(d p=209 \mu \mathrm{m}, X=$ $20 \%, C=T / 8, N=300 \mathrm{rpm})$.

\subsubsection{Axial Particle Concentration Profiles}

To assess the distribution of the solid particles in the mixing vessel, Equation (3.4-4) was employed to quantify the extent of homogeneity. The resulting solid volume fractions $\left(X_{v}\right)$ calculated using Equation (3.4-3) were normalised with respect to the average solid volume fraction $\left(\bar{X}_{v}\right)$ to obtain the particle concentration profiles. Figure (5.1-5) demonstrates the axial particle concentration profiles for the Maxblend impeller as a function of the agitation speed. As $X_{v}$ approaches $\bar{X}_{v}$, homogeneity inside the tank increases pointing out the uniform distribution of particles in the mixing vessel. When $X_{v} / \bar{X}_{v}$ becomes 1 , the extent of homogeneity reaches its maximum value of 1 . 


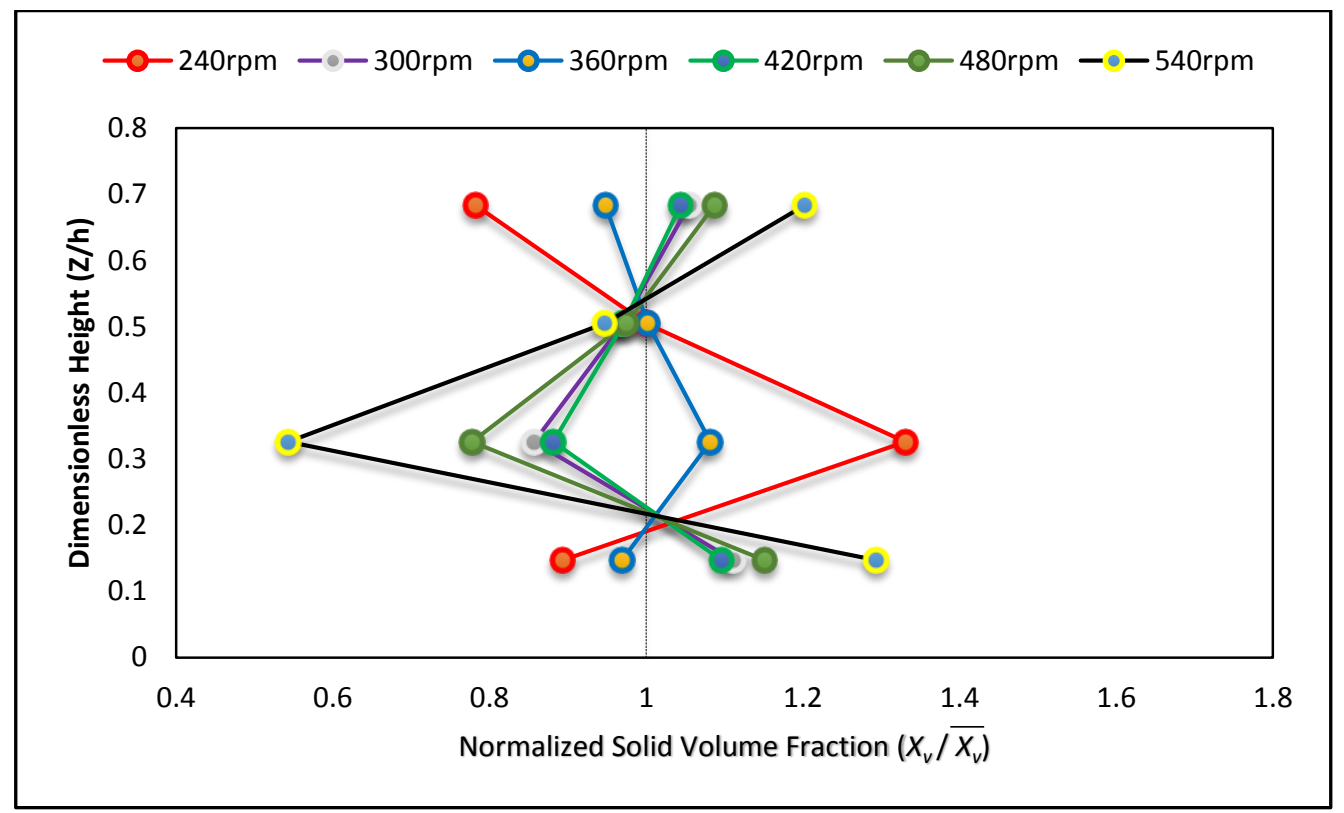

Figure (5.1-5). Axial particle concentration profile at different agitation speeds (Maxblend Impeller, $C=T / 8, X=10 \mathrm{wt} \%$, and $\left.d_{p}=537 \mu \mathrm{m}\right)$.

\subsubsection{Effect of Agitation Speed}

It is well understood that the agitation speed significantly affects the solid-liquid mixing operation. The effects of agitation speed on extent of homogeneity for both baffled and unbaffled Maxblend impeller were investigated and analyzed in this study. Figure (5.1-6) demonstrates the degree or extent of homogeneity at different stirrer speeds for both baffled and unbaffled conditions. As can be evident in Figure (5.1-6), the extent of homogeneity in the mixing vessel was enhanced when the stirrer speed for both baffled and unbaffled conditions increased. When the level of homogeneity approached the peak value, further increment in the stirrer speed had a detrimental impact on homogeneity for both baffled and unbaffled conditions. Similar phenomenon has been reported in some research works for the fully baffled mixing vessels (Bohnet and Niesmak, 1980; Hosseini et al., 2010a; Tahvildarian et al., 2011). Hosseini et al. (2010a) reported that the formation of the zones with a lower particle concentration at the higher stirrer speeds was responsible for the reduction in homogeneity for the fully baffled vessels because of the centrifugal force in the circulation loop. Mak (1992) also suggested a decrease in the level of homogeneity in the slurry reactors beyond the optimum stirrer speed for the fully 
baffled conditions. Hence, the assessment of the optimum impeller speed is extremely important to enhance the local mixing quality in the mixing vessel.

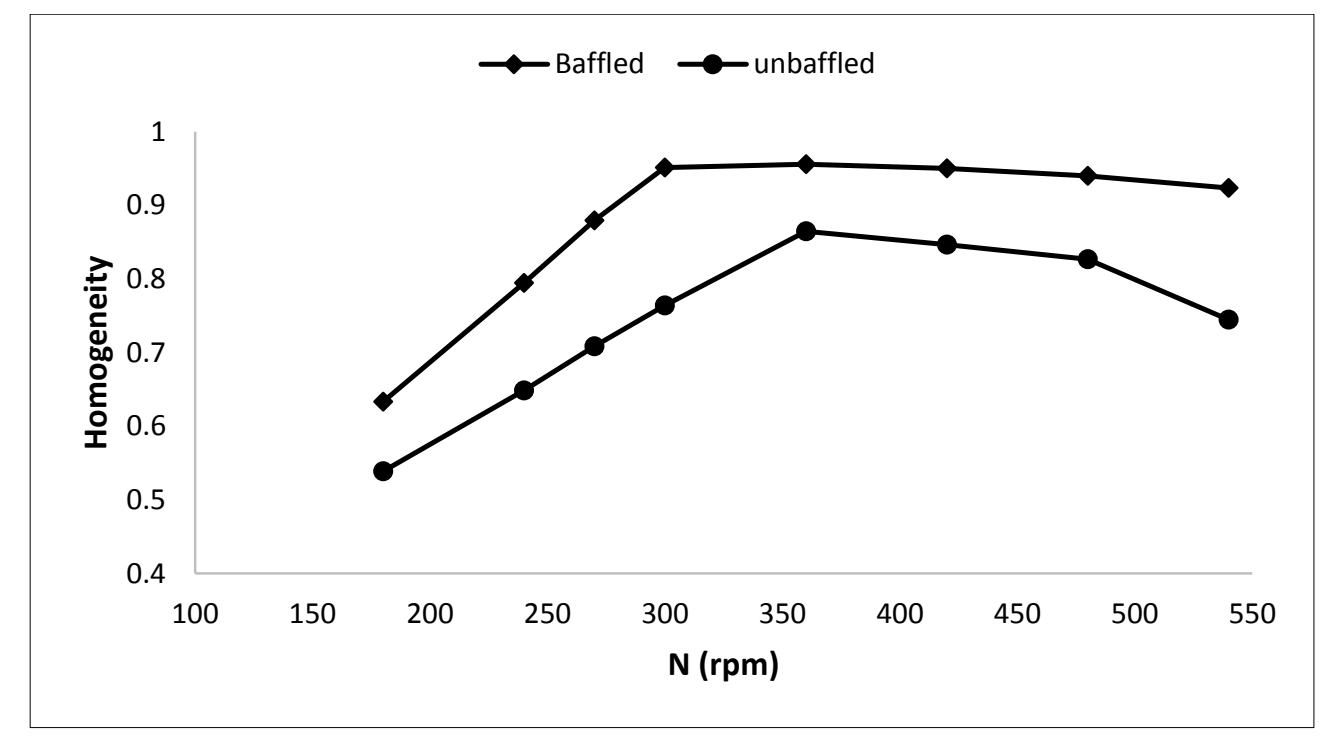

Figure (5.1-6). Effect of the agitation speed on the degree of homogeneity attained with baffled and unbaffled Maxblend impeller $(\mathrm{C}=T / 8, X=10 \mathrm{wt} \%$, and $d p=537 \mu \mathrm{m})$.

\subsubsection{Effect of baffles}

As a close clearance impeller, the Maxblend impellers can be employed with and without baffles in mixing operations. Thus, the effect of use of baffles for the Maxblend impeller on the suspension of the solid particles was analyzed in this paper. As can be observed in Figure (5.1-6), the maximum level of homogeneity attained for the baffled vessel was significantly higher than that for the unbaffled vessel at the same stirrer speed. Baffles are basically installed to prevent the formation of dead zones and vortex, and to control the flow inside the mixing tank (Paul et al., 2004). Due to formation of dead zones and vortex, the solid particles were not distributed uniformly throughout the unbaffled mixing tank. It is imperative to mention that the homogeneity level significantly decreased beyond optimum stirred speed in an unbaffled vessel.

The effect of baffles on the extent of homogeneity with respect to the power consumption was also explored in this study. Figure (5.1-7) illustrates the effect of power consumption on the level and degree of homogeneity for baffled and unbaffled mixing vessels furnished with the Maxblend impeller. The maximum level of homogeneity attained for the baffled vessel was 
significantly higher than that for the unbaffled vessel at the same power consumption. The use of baffles significantly enhanced the local mixing quality inside the mixing vessel. It should be noted that the use of baffles improves the mixing quality with an efficient and effective usage of power. It can be concluded that baffled mixing vessels are better choice to achieve the higher mixing quality at a lower power consumption.

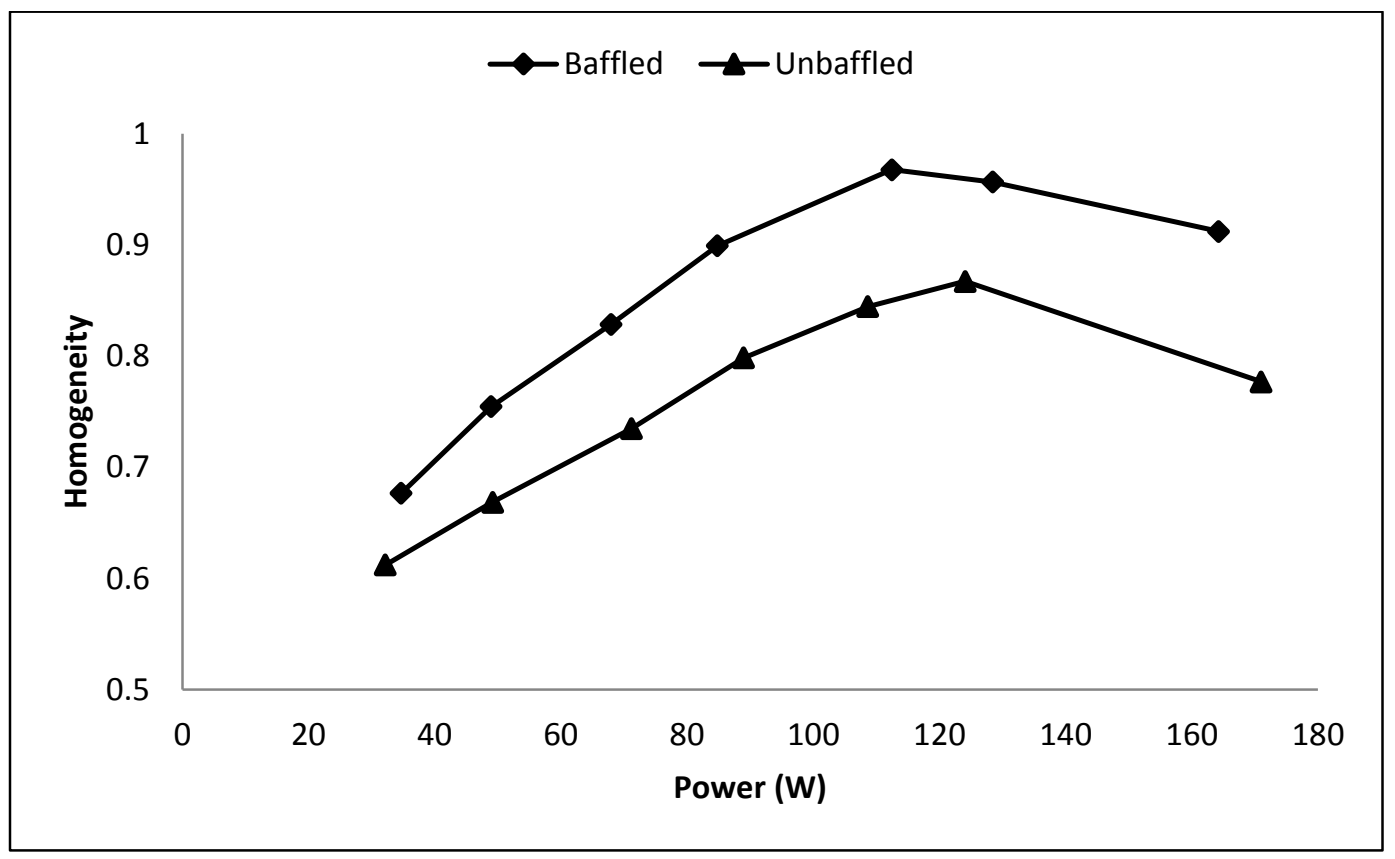

Figure (5.1-7). Effect of power on homogeneity for the baffled and unbaffled Maxblend impeller $\left(C=T / 8, X=10 \mathrm{wt} \%, d_{p}=752 \mu \mathrm{m}\right)$.

\subsubsection{Just Suspended Agitation Speed}

It is essential to determine the just suspended agitation speed $\left(N_{j s}\right)$, which is defined as the stirrer speed required for the complete suspension in the solid-liquid mixing operations. The correlation proposed by Zwietering (1958) (see Equation (2.4-1)) could not be employed for this study because the dimensionless number $(S)$, which is a function of the system geometry, was not known for the Maxblend impeller. Thus, the power number $\left(P_{o}\right)$ technique adopted by Raghava Rao et al. (1988) and Tahvildarian et al. (2011) was utilized to evaluate the just suspended agitation speed $\left(N_{j s}\right)$ in this study for the Maxblend impeller. It has been reported that this technique provides reliable and accurate estimation of the just suspended agitation speed $\left(N_{j s}\right)$. To estimate $N_{j s}$ through 
this method, the stirrer speed was increased gradually at a constant solid loading and the measurement of the stirrer power was taken simultaneously at each agitation speed. More solid particles got suspended in the system with an increase in the agitation speed, which eventually increased the power number $\left(P_{o}\right)$. The power number $\left(P_{o}\right)$ became constant once the complete suspension state was attained. The agitation speed at which $P_{o}$ remained constant was the just suspended agitation speed. Figure (5.1-8) shows the estimation of the just suspended agitation speed $\left(N_{j s}\right)$ in this paper. The just suspended agitation speed $\left(N_{j s}\right)$ was found to be $3.4 \mathrm{rps}(204$ rpm) according to this graph.

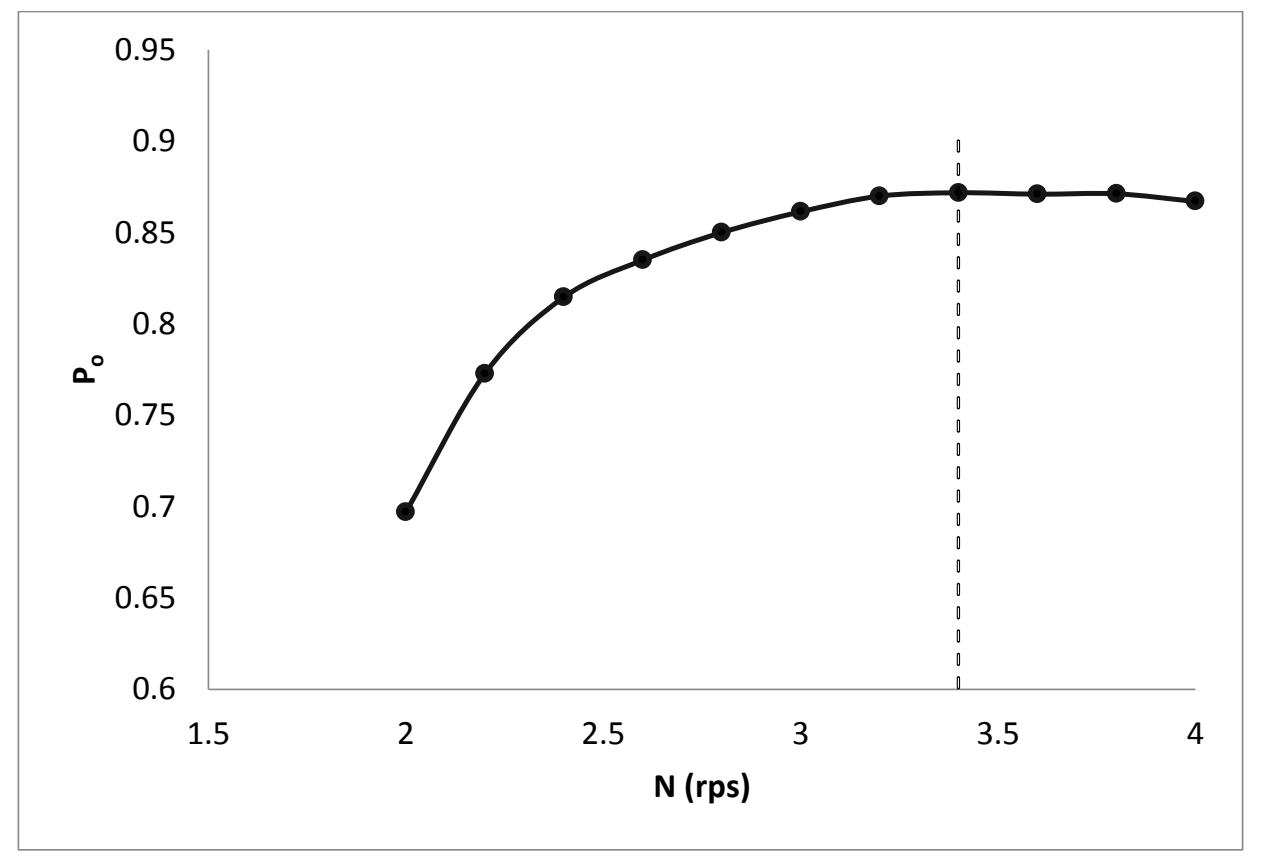

Figure (5.1-8). Estimation of just-suspended speed using the impeller power (Maxblend Impeller, $\left.C=T / 8, X=10 \%, d_{p}=209 \mu \mathrm{m}\right)$.

\subsubsection{Effect of solid loading}

The solid particle loading significantly influences the mixing quality in the solid-liquid mixing operations. Figure (5.1-9) illustrates the degree of homogeneity with respect to the stirrer speed at three solid loadings (5 wt $\%, 20 \mathrm{wt} \%$, and $30 \mathrm{wt} \%)$. When the solid loading increased, the stirrer speed at which the highest level of homogeneity took place increased as well. When the solid loading increases, the density as well as the viscosity of the slurry increase. Thus, to distribute 
the particles uniformly throughout the mixing vessel, a higher agitation speed is desired. Since increasing of the solid loading results in an increase in the number of particles in the fluid, the hindered settling velocity must be considered instead of the free settling velocity at the higher solid concentrations. According to Paul et al. (2004), the hindered settling velocity is developed due to: (a) the interaction among particles (b) increasing of viscosity and density of the slurry, and (c) the contact and interaction of settling solid particles with the upward flowing liquid. Maude and Whitmore (1958) developed the following equation for the hindered settling velocity:

$$
V_{t s}=V_{t}\left(1-X_{v}\right)^{n_{t}}
$$

where $V_{t}$ and $V_{t s}$ are settling velocity and hindered settling velocity of solid particles, respectively. $X_{v}$ is the solid volume fraction and $n_{t}=2.33$ for $R e_{P}>1000, n_{t}=4.375 \operatorname{Re}_{P}^{-0.0875}$ for $0.3<$ $R e_{P} \leq 1000$ and $n_{t}=4.64$ for $R e_{P} \leq 0.3$, where $R e_{P}$ is the particle Reynolds number. The uniform dispersion and distribution of solid particles increases at the higher particle concentration thus increasing the homogeneity level. The results presented in Figure (5.1-9) show that the highest degree of homogeneity was attained at the highest solid loading of $30 \mathrm{wt} \%$ due to the impact of the hindered settling velocity. Similar observation have been mentioned in the previous research works by Godfrey and Zhu (1994) and Hosseini et al. (2010). However, Tahvildarian et al. (2011) reported that the influence of the hindered settling velocity at the higher solid loading was not pronounced for the average particle sizes less than 10 microns when the density of the solid particle was almost equal to the density of liquid medium in their study. 


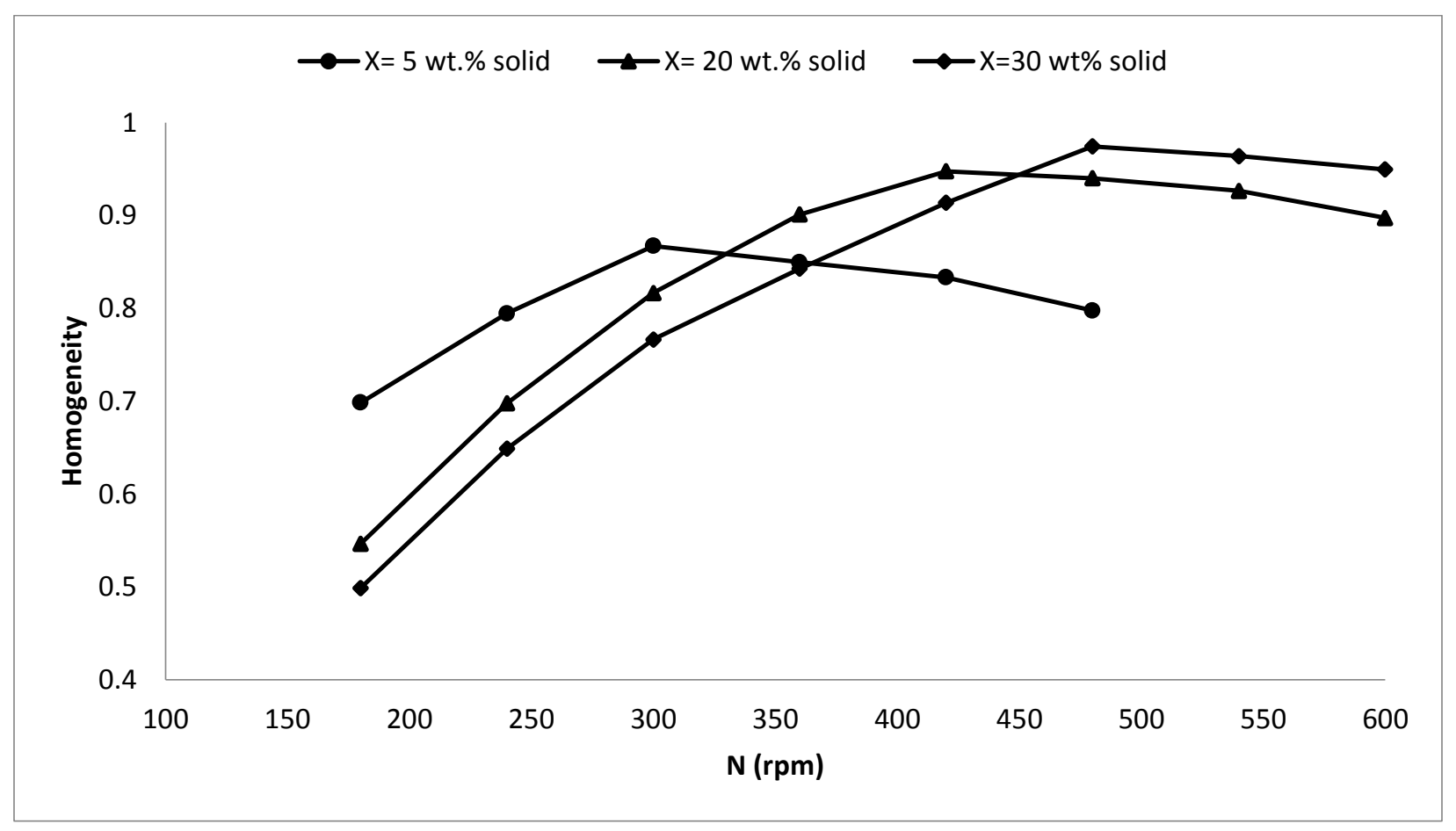

Figure (5.1-9). Effect of the particle concentration on the level of homogeneity at different agitation speeds (Maxblend Impeller, $C=T / 8$, and $d_{p}=752 \mu \mathrm{m}$, Max. std. dev. $=1.38 \%$ ).

\subsubsection{Comparison of Mixing Indices}

To understand the mixing performance of the Maxblend impeller in the solid-liquid mixing operation precisely, the effect of the stirrer speed on different mixing indices was studied. The homogeneity considered in this study so far gives axial particle concentration profiles. However, Michelletti et al. (2003) suggested that the effect of the radial solid concentration profile should be taken into considerations while studying the distribution of solid particles in slurries. A new index $\left(M I_{r}\right)$ was used by Williams et al. (1996) to assess the local mixing quality in the mixing vessel. They first calculated the relative standard deviation $\left(M I_{z}\right)$ with respect to each plane as:

$$
M I_{z}=\frac{1}{\bar{\sigma}_{i}} \sqrt{\sum_{1}^{n} \frac{\left(\sigma_{e}-\bar{\sigma}_{l}\right)^{2}}{n-1}}
$$


where $\bar{\sigma}_{i}, \sigma_{e}$ and $n$ are average conductivity over the given plane, conductivity value in each pixel, and the number of pixel on the selected plane. They then calculated the mixing index $\left(M I_{r}\right)$ by taking average of $M I_{z}$ over all selected planes as:

$$
M I_{r}=\frac{\sum_{1}^{q} M I_{z}}{q}
$$

To assess the concentration profiles in the radial direction, mixing index proposed by Williams et al. (1996) was considered in this study.

To obtain an estimate of the local mixing quality, Carletti et al. (2014) proposed a new mixing index $\left(M I_{o}\right)$, which can be determined as:

$$
M I_{o}=\frac{1}{\bar{\sigma}} \sqrt{\sum_{1}^{p_{t}} \frac{\left(\sigma_{e}-\bar{\sigma}\right)^{2}}{p_{t}-1}}
$$

where $\bar{\sigma}$ and $p_{t}$ are the mean conductivity value and total number of pixels on the whole selected volume of the tank. The total number of pixels in this study was 1264 . The mixing index $\left(M I_{o}\right)$ proposed by Carletti et al. (2014) was used in this study to assess the local mixing quality. The following correlation as employed by Carletti et al. (2014) was used to compare the results with the degree of homogeneity adopted by Hosseini et al. (2010a):

$$
M I_{v}=\frac{1}{\bar{\sigma}} \sqrt{\sum_{1}^{q} \frac{\left(\bar{\sigma}_{l}-\bar{\sigma}\right)^{2}}{q-1}}
$$

where $M I_{v}$ is the mixing index and $q$ is the number of planes. Figure (5.1-10) demonstrates the comparison of three aforementioned mixing indices with respect to the stirrer speed. It can be 
noted that the number of points selected for the calculation of $M I_{o}$ are significantly higher than those taken for the estimation of $M I_{v}$. As can be seen in Figure (5.1-10), $M I_{r}$ reduced slowly with an increase in the stirrer speed whereas $M I_{v}$ and $M I_{o}$ decreased remarkably with an increase in the stirrer speed indicating that the effect of the radial particle gradients were considerably less in the solid-liquid system. Carletti et al. (2014) observed the similar phenomenon using the PBT impeller. $M I_{r}$ as suggested by Harrison et al. (2012) does not take into account the effect of axial particle gradients, hence it provides the mixing quality in terms of the radial homogeneity. It can be concluded that the axial particle concentration gradients are more pronounced compared to the radial particle gradients in the system.

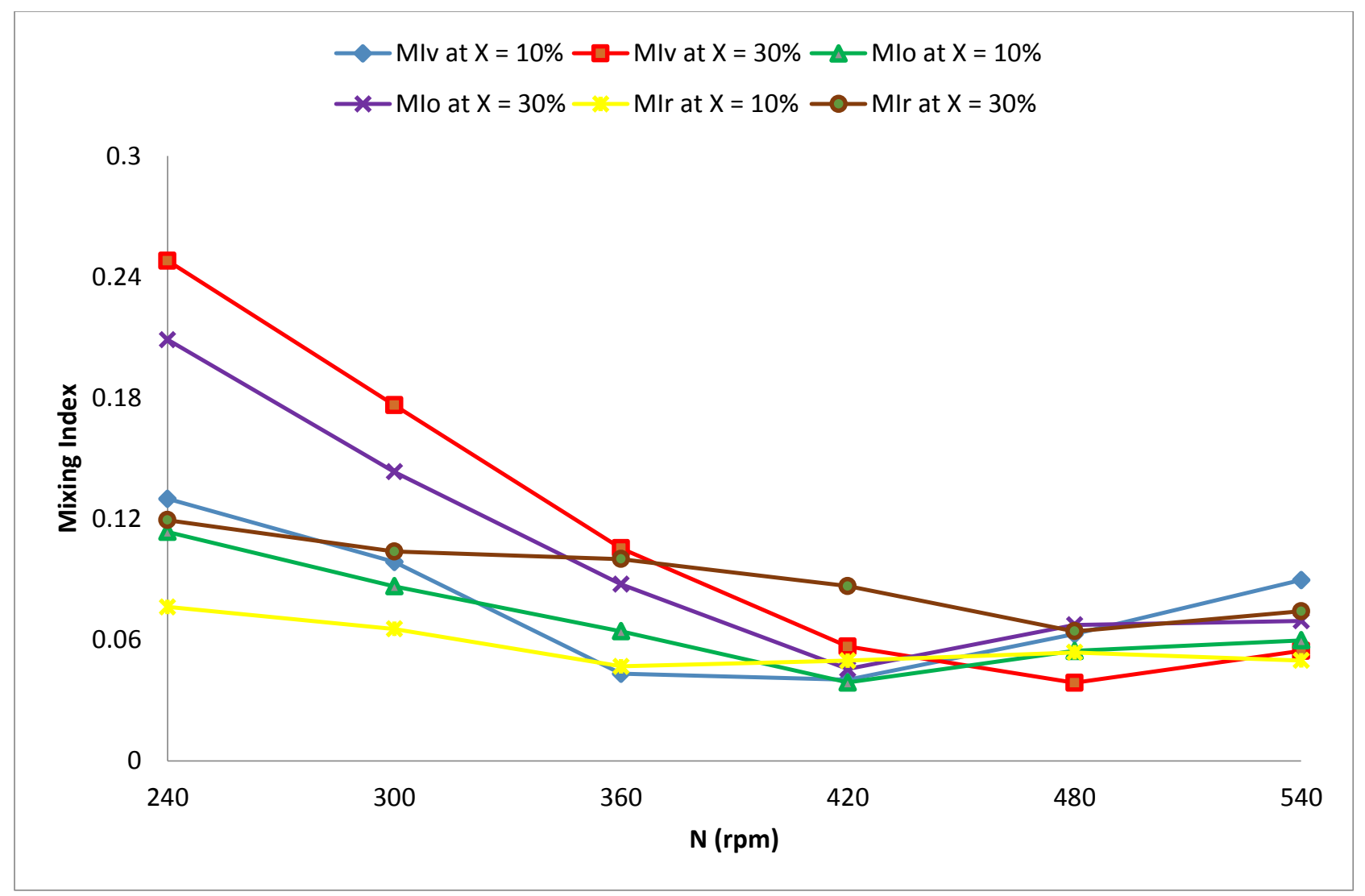

Figure (5.1-10). Effect of the impeller speed on mixing indices at different solid loadings (Maxblend impeller, $C=T / 8, d_{p}=209 \mu \mathrm{m}$, Max. std. dev. $=1.29 \%$ ). 


\subsubsection{Effect of average particle size}

Physical properties of the solid particles particularly the size of the particles greatly influence the solid-liquid suspensions. Figure (5.1-11) depicts the influence of the particle diameter (size) on the level of homogeneity in the mixing vessel. The results clearly show that the smallest solid particles (209 microns) employed in this investigation attained the highest homogeneity level. These results are consistent with the results reported by several researchers (Godfrey and Zhu, 1994; Pekker and Helvaci, 2008; Hosseini et al., 2010a; Tahvildarian et al., 2011). Perry and Green (1984) developed the following equation to determine the free settling velocity of spherical particles:

$$
V_{t}=\left[\frac{4 g_{c} d_{p}\left(\rho_{s}-\rho_{l}\right)}{3 C_{D} \rho_{l}}\right]^{1 / 2}
$$

where $V_{t}, g_{c}, d_{p}, \rho_{s}, \rho_{l}$, and $C_{D}$ are terminal settling velocity, gravitational constant, diameter of solid particle, density of particle, density of liquid, and the drag coefficient, respectively. According to equation (5.1-6), an increment in solid particle diameter (size) increases the terminal settling velocity of the solid particles. Therefore, the finer solid particles settle more slowly than the larger ones. In fact, the fine particles would be easily suspended in comparison to the large solid particles.

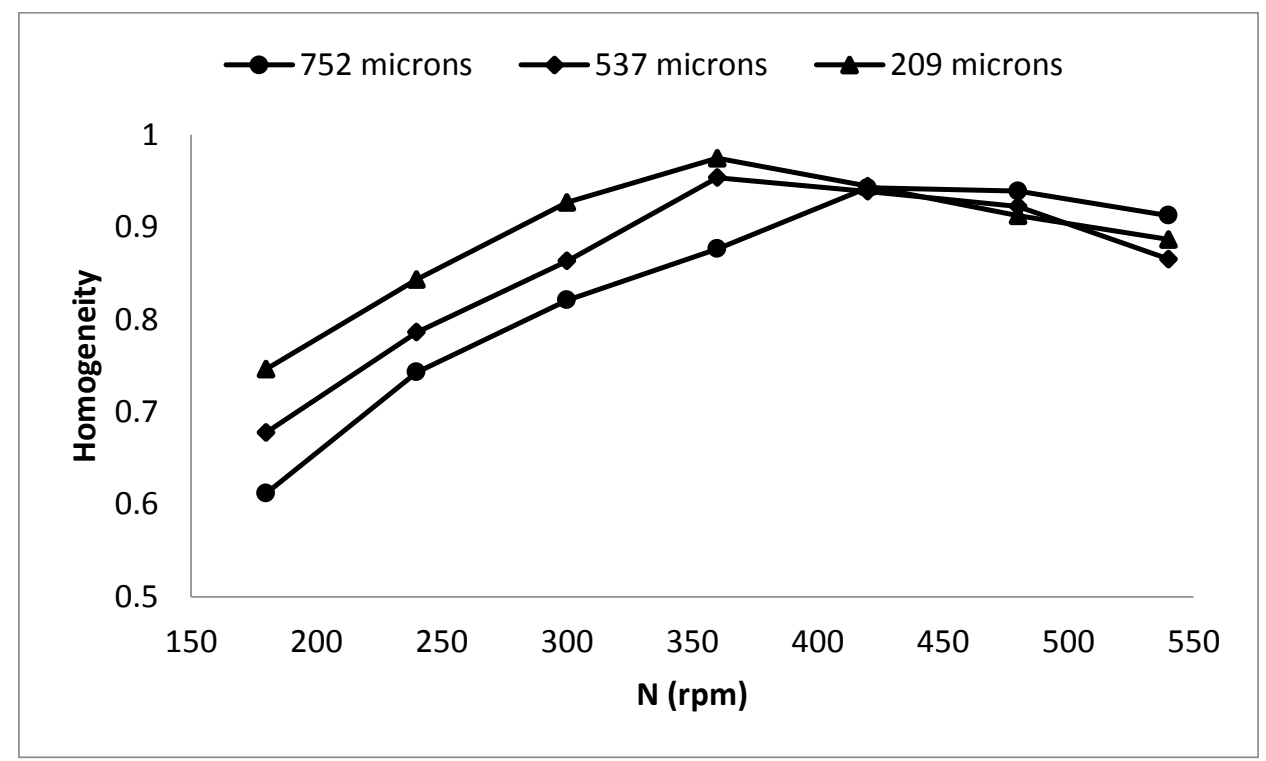

Figure (5.1-11). Influence of the particle diameter on the degree of homogeneity at different agitation speeds (Maxblend Impeller, $C=T / 6, X=10 \mathrm{wt} \%$, Max. std. dev. $=1.09 \%$ ). 


\subsubsection{Influence of Stirrer Type}

The main objective of this study was to assess the performance of the Maxblend impeller for solid-liquid mixing operations. To achieve this goal, the efficiency of the Maxblend impeller was compared to the efficiencies of the A200 (an axial-flow impeller) and Rushton (a radial-flow impeller). In fact, the A200 and Rushton impellers have been widely used by other researchers for solid-liquid mixing operations (Hosseini et al., 2010a; Tahvildarian et al., 2011; Harrison et al., 2012). The axial-flow impellers are preferred over the radial-flow impellers for the solid-liquid mixing (Paul et al., 2004). Different impellers have different torques at the same agitation speed because the surface areas were different for these impellers. The Maxblend impeller had a large surface area compared to the A200 (axial) and Rushton (radial) impellers and hence, the degree of homogeneity for all three impellers were compared in terms of power consumption. Several research works have been reported in the literature for comparison of the mixing performance of the different impellers in terms of power consumption (Hosseini et al., 2010a; Tahvildarian et al., 2011). Figure (5.1-12) shows the degree or level of homogeneity with respect to the power consumption for the above mentioned three impellers. It can be observed that the maximum level of homogeneity attained by the Maxblend impeller was significantly higher than those achieved by the other two impellers. These results clearly demonstrate that the Rushton turbine impeller is not suitable for the solid-liquid mixing operations. In fact, the mixing quality of the solid-liquid systems can be enhanced by the use of the Maxblend impeller. It should be also noted that the power essential to achieve the maximum level of homogeneity by the Maxblend impeller was lower than that of the A200 impeller even though the maximum homogeneity achieved by the Maxblend impeller was significantly higher (Figure 5.1-13). Our results demonstrated that the Maxblend impeller was more efficient than the A200 and Rushton impellers in slurry reactors. 


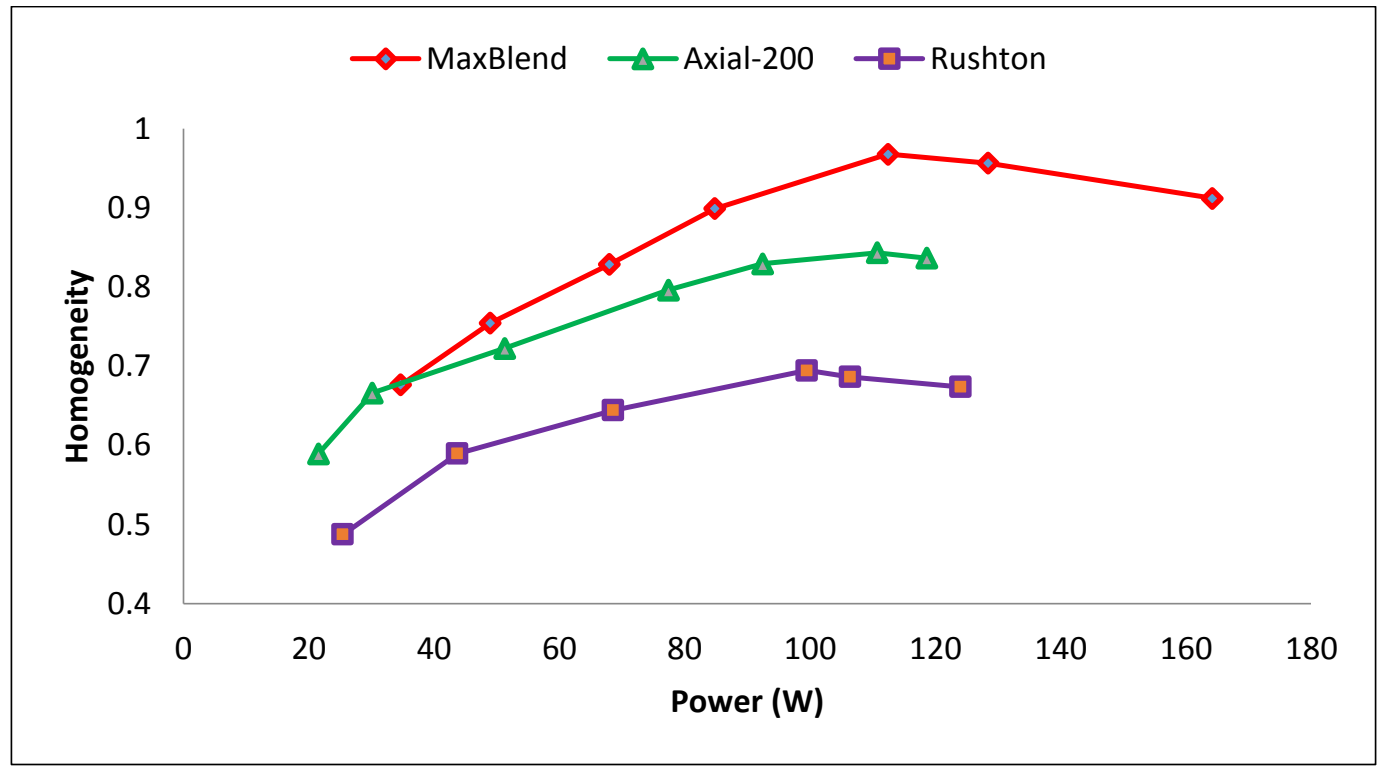

Figure (5.1-12). Effect of the power consumption on homogeneity for different types of impellers $\left(C=T / 8, X=10 \mathrm{wt} \%, d_{p}=752 \mu \mathrm{m}\right)$.

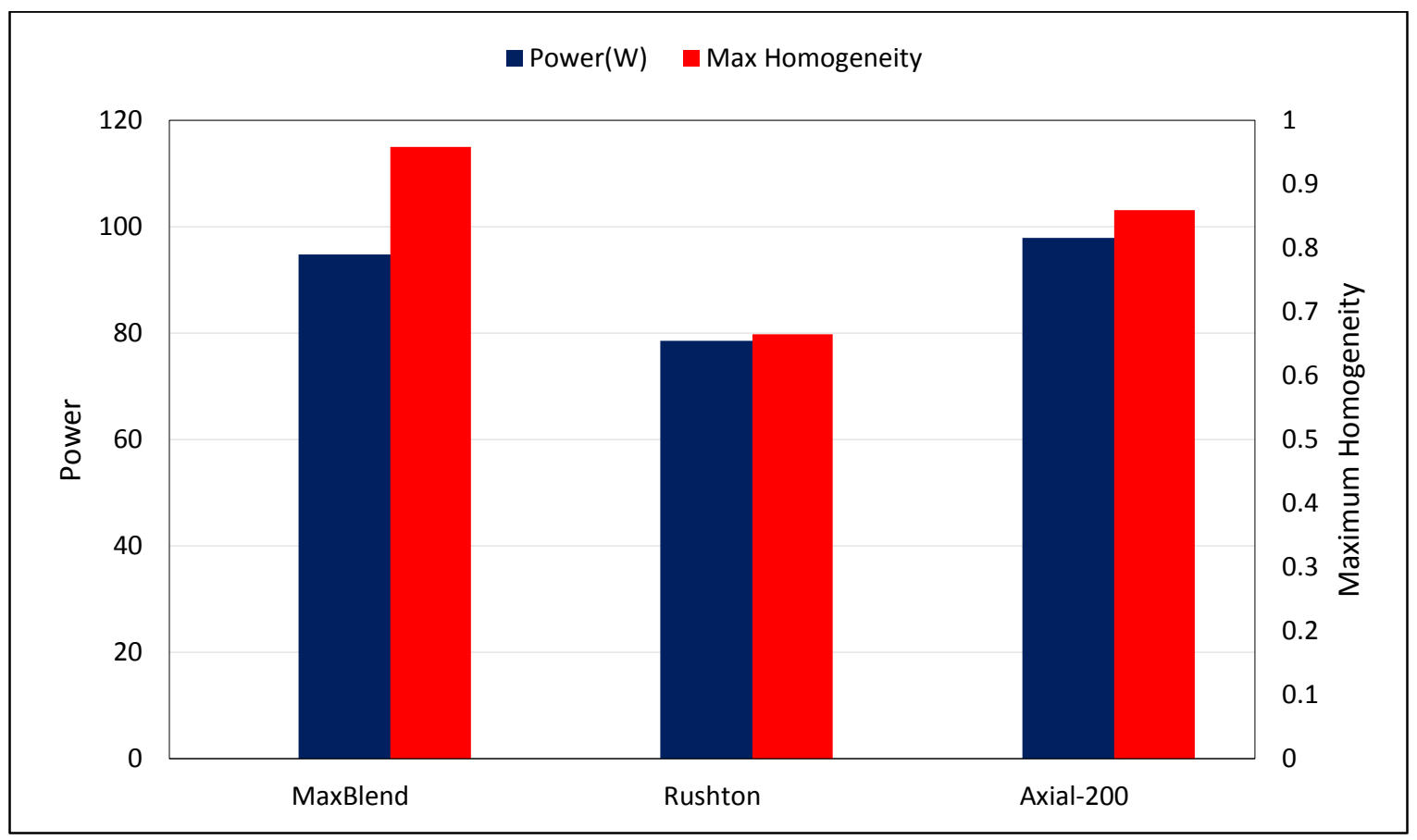

Figure (5.1-13). Power required for different impellers to attain maximum homogeneity $(C=T / 6$, $X=10 \mathrm{wt} \%$, and $\left.d_{p}=209 \mu \mathrm{m}\right)$. 


\subsubsection{Effect of Stirrer Clearance}

The stirrer off-bottom clearance has a pronounced impact on the solid-liquid mixing quality. Three different stirrer clearances of $C=T / 8, T / 6$, and $T / 4$ were investigated for the Maxblend impeller in this study. Figure (5.1-14) illustrates the effect of the off-bottom clearance upon the level of homogeneity at various stirrer speeds. As can be seen in this figure, the homogeneity level increased with a decrement in the off-bottom clearance from $C=T / 4$ to $C=T / 8$ and the highest level of homogeneity was obtained at $C=T / 8$. By increasing the off-bottom clearance, the capability of the stirrer to suspend the particles decreased due to the reduction in energy dissipation and change in flow pattern (Kresta and Wood, 1993). As a matter of fact, the flow profile and pattern generated by the stirrer changed basically from the axial to the radial flow pattern with the increase in stirrer off-bottom clearance, which ultimately reduced the extent of homogeneity within the system (Montante et al., 1999). Similar phenomenon was reported by Armenante and Nagamine (1998) at the impeller clearance greater than $T / 2.8$ equipped with PBT impellers. Although no information has been reported regarding the impact of the clearance of the Maxblend impeller on the solid-liquid mixing operation, Ameur et al. (2012) observed the formation of a secondary loop while increasing the impeller clearance in the mixing of the yield stress fluids. The secondary loop vanished when off-bottom clearance was decreased, improving down and up movement remarkably. Hosseini et al. (2010a) and Tahvildarian et al. (2011) observed the maximum homogeneity level at $C=T / 3$ for the solid-liquid systems equipped with A310 impeller. Hicks et al. (1996) observed the best mixing performance at $C=T / 2.5$ for the solid-liquid mixing with the PBT impellers. It should be noted that the desired agitation speed to attain the highest

level of homogeneity was entirely independent of the stirrer location for the defined range of the off-bottom clearance in this study. 


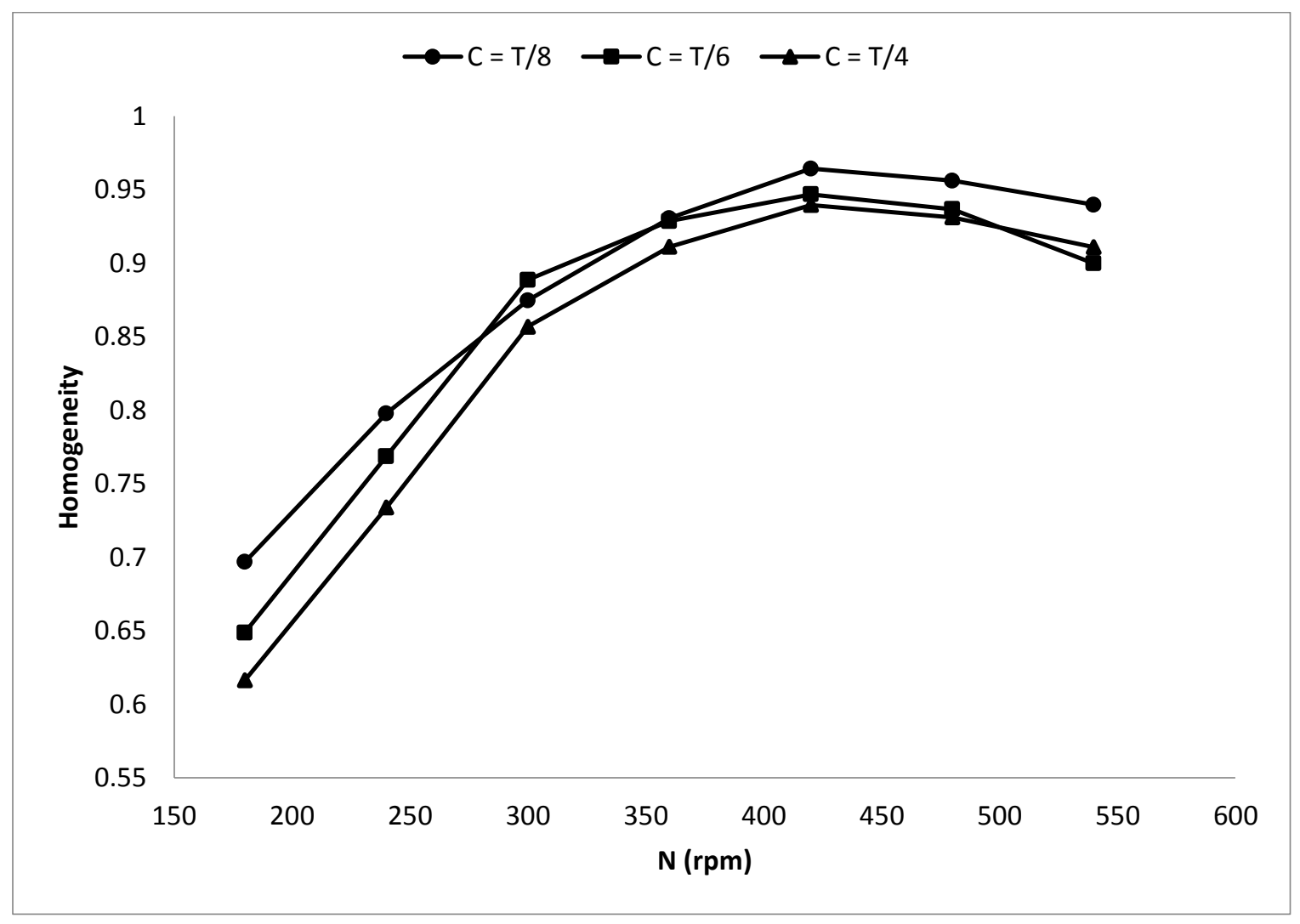

Figure (5.1-14). Effect of the stirrer off-bottom clearance on the level of homogeneity at various agitation speeds (Maxblend Impeller, $X=$ wt $20 \%$ and $d_{p}=209 \mu \mathrm{m}$, Max. std. dev. $=1.03 \%$ ). 


\subsection{Using Computational Fluid Dynamics to Analyze the Performance of the Maxblend Impeller in Solid-Liquid Mixing Operations}

Development of CFD modeling for this study was discussed in details in Section 4. All the results are presented and discussed for baffled conditions unless unbaffled conditions are mentioned. Specific gravity (SG) of particle was considered to be 2.5 for all the results unless mentioned otherwise. It is imperative to mention that the validation of the generated CFD model was done by comparing the results obtained from the simulation to experimental data in terms of stirrer torque, just suspension speed $\left(N_{j s}\right)$ and the degree of homogeneity.

\subsubsection{Qualitative Validation}

In order to validate the CFD model, four planes (corresponding to four tomographic planes) were created to compare the particle distribution inside the stirred vessel at $N=420 \mathrm{rpm}$ (Figure 5.2-1a). As can be evident from the Figure (5.2-1a), the solid particles were uniformly suspended inside the stirred tank at $N=420 \mathrm{rpm}$. In order to understand the solid particles distribution for the non-homogeneous system precisely, the results obtained from the CFD simulation was compared to those retrieved from the ERT measurements at $N=240 \mathrm{rpm}$ (Figure 5.2-1b). As can be seen in the Figure (5.2-1b), the dispersion of the particles were not uniform inside the stirred vessel at $N$ $=240 \mathrm{rpm}$. The results from the CFD model are consistent with the results from the conductivity tomograms. 


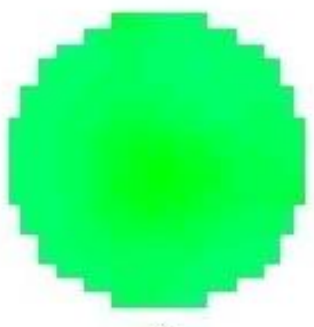

P1

$420 \mathrm{rpm}$

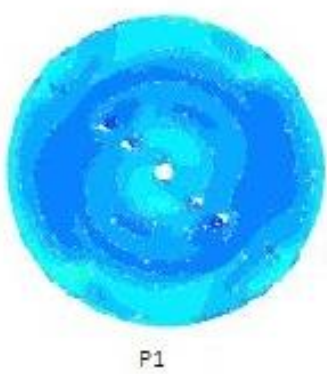

$420 \mathrm{rpm}$

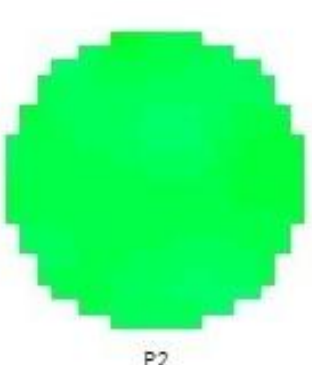

P2

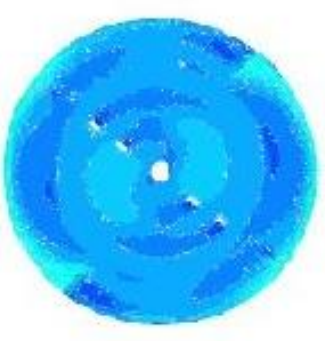

P2

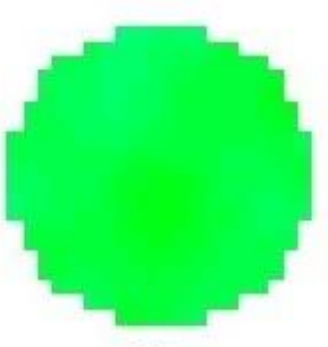

P3

(a)

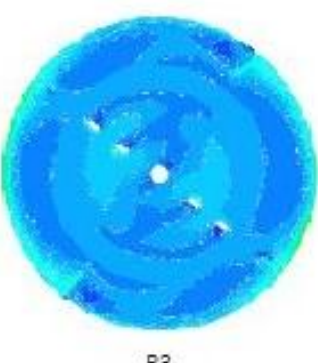

P3

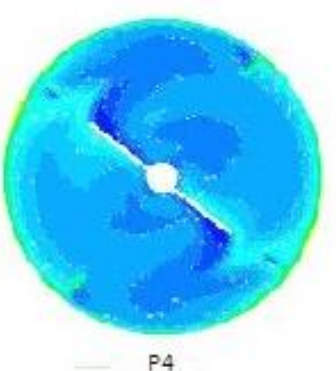

(b)

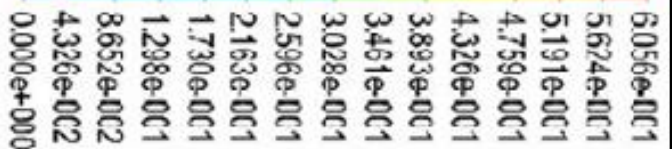

Figure (5.2-1a). (a) Conductivity tomograms, and (b) particle concentration contours created by CFD for four different horizontal planes (Maxblend impeller, $d_{p}=209 \mu \mathrm{m}, X=10 \mathrm{wt} \%, C=T / 8$, and $N=420 \mathrm{rpm}$ ). 


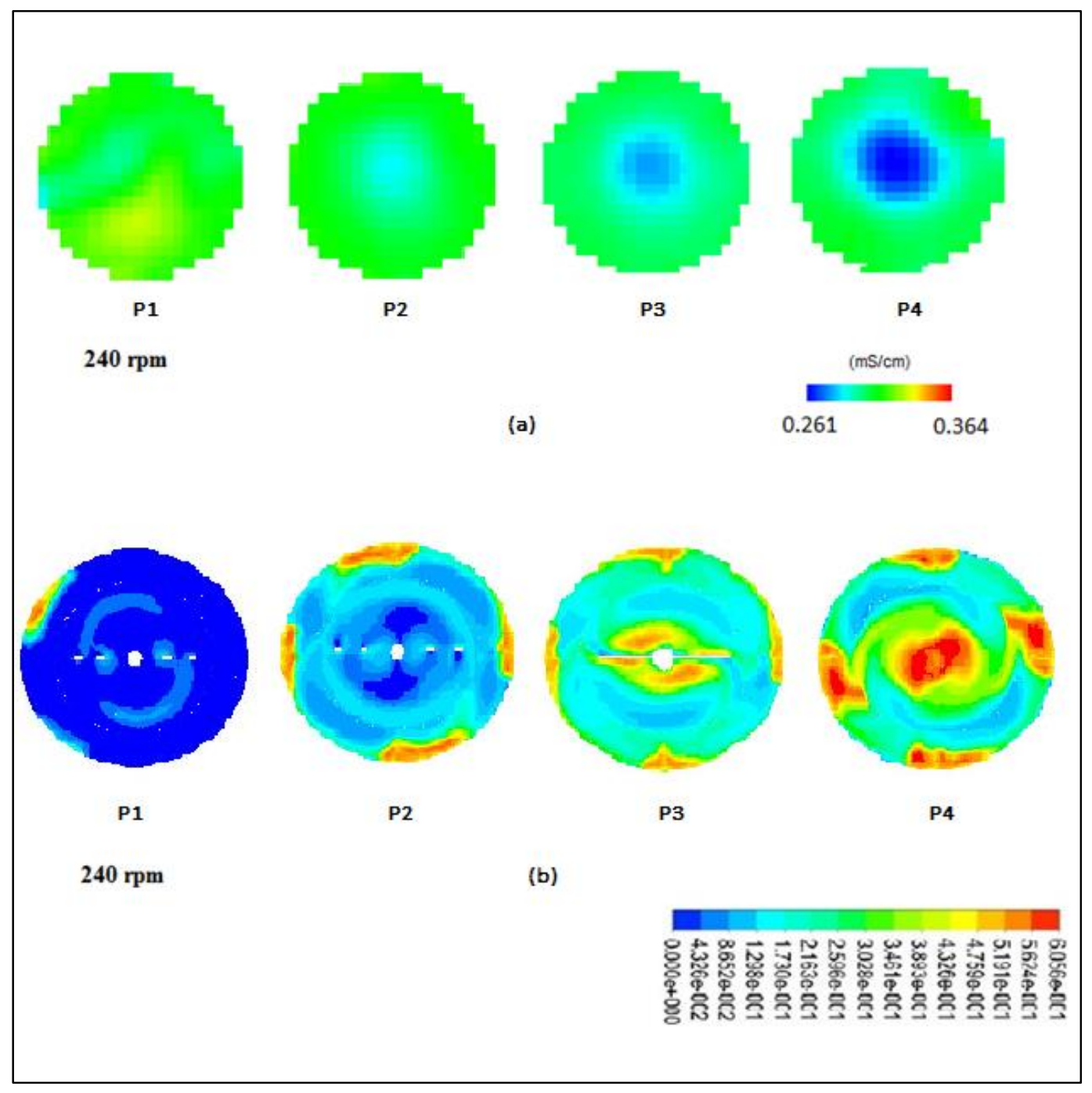

Figure (5.2-1b). (a) Conductivity tomograms, and (b) particle concentration contours created by CFD for four different horizontal planes (Maxblend impeller, $d_{p}=752 \mu \mathrm{m}, X=20 \mathrm{wt} \%, C=T / 8$, and $N=240 \mathrm{rpm})$.

\subsubsection{Stirrer Torque}

Figure (5.2-2) illustrates the stirrer torque with respect to the stirrer speed for the Maxblend impeller. Significant change in the stirrer torque was evident as the particles were suspended inside the stirred vessel for both experimental and computed results. The computed torque was consistent with the experimentally calculated torque. The standard deviation value between computed and measured torque was noticed to be less than $1 \%$. 


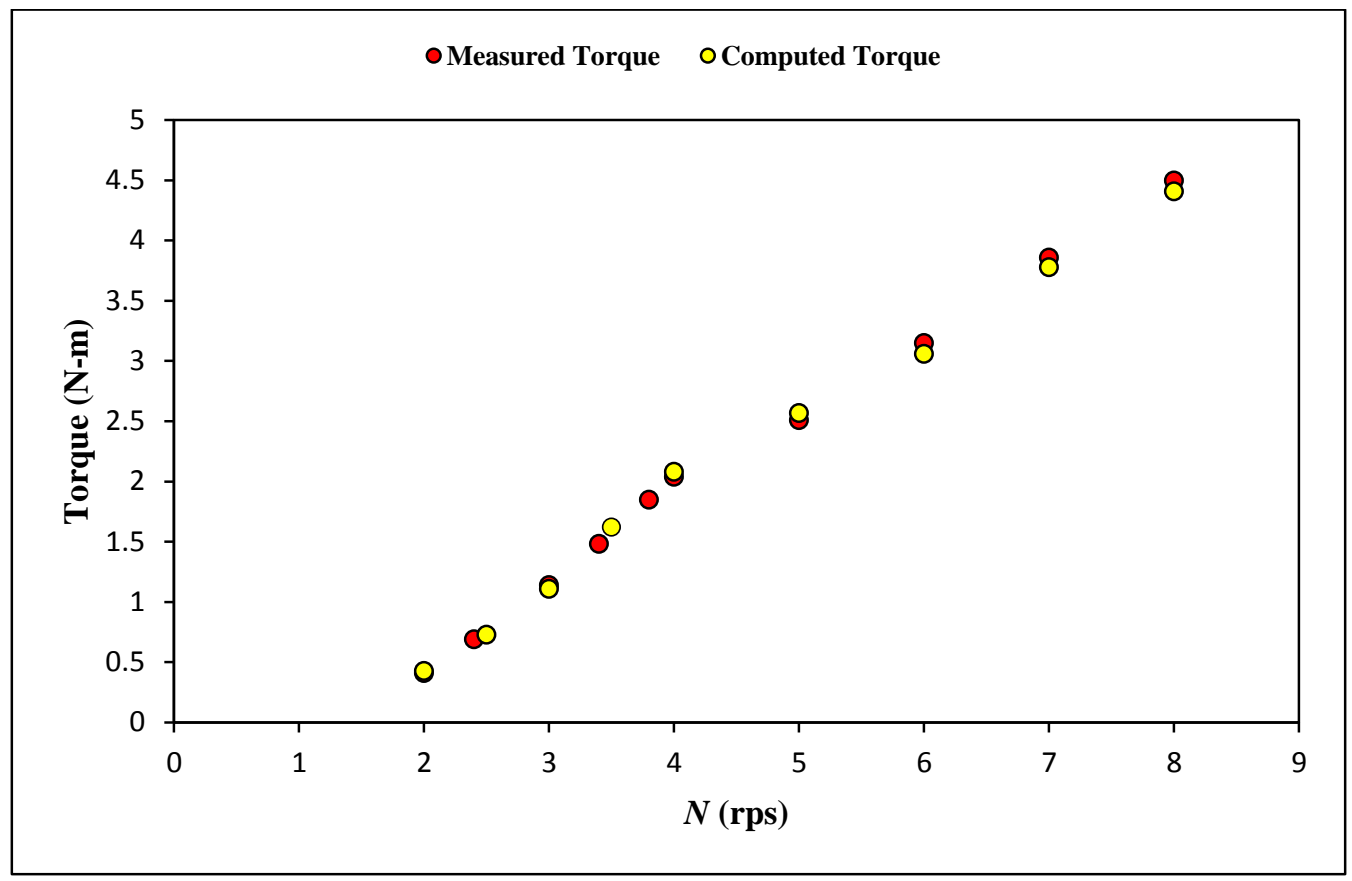

Figure (5.2-2). Stirrer torque versus agitation speed for the Maxblend impeller $\left(d_{p}=209 \mu \mathrm{m}, X=\right.$ $10 \mathrm{wt} \%$, and $C=T / 8)$.

\subsubsection{Just Suspension Speed}

Several researchers have adopted the just suspension speed $\left(N_{j s}\right)$ method to examine the mixing quality in slurry reactors. The just suspension speed $\left(N_{j s}\right)$ is defined as the agitation speed needed to attain the complete (off-bottom) suspension inside the slurry reactors. The average solid volume fraction was measured at different agitation speeds at $1.5 \mathrm{~mm}$ above the base of the mixing vessel for a horizontal plane by employing the CFD model to determine $N_{j s}$ (Figure 5.2-3). Two tangents (one at the point of minimum slope and another at the point of maximum slope) were drawn to the curve as shown in the Figure (5.2-3). The just suspension speed $\left(N_{j s}\right)$ was the stirrer speed in rpm corresponding to the intersection point of these tangents. Mak (1992) and Hosseini et al. (2010b) employed the similar approach to estimate $N_{j s}$. The just suspension speed $\left(N_{j s}\right)$ was found to be $215 \mathrm{rpm}$ using this method for the Maxblend impeller. The power number $(\mathrm{Po})$ method was employed to determine $N_{j s}$ from the experimental data. $N_{j s}$ was estimated to be $204 \mathrm{rpm}$ at the similar conditions for the Maxblend impeller. The relative error between these two $N_{j s}$ values was found to be $5 \%$. 


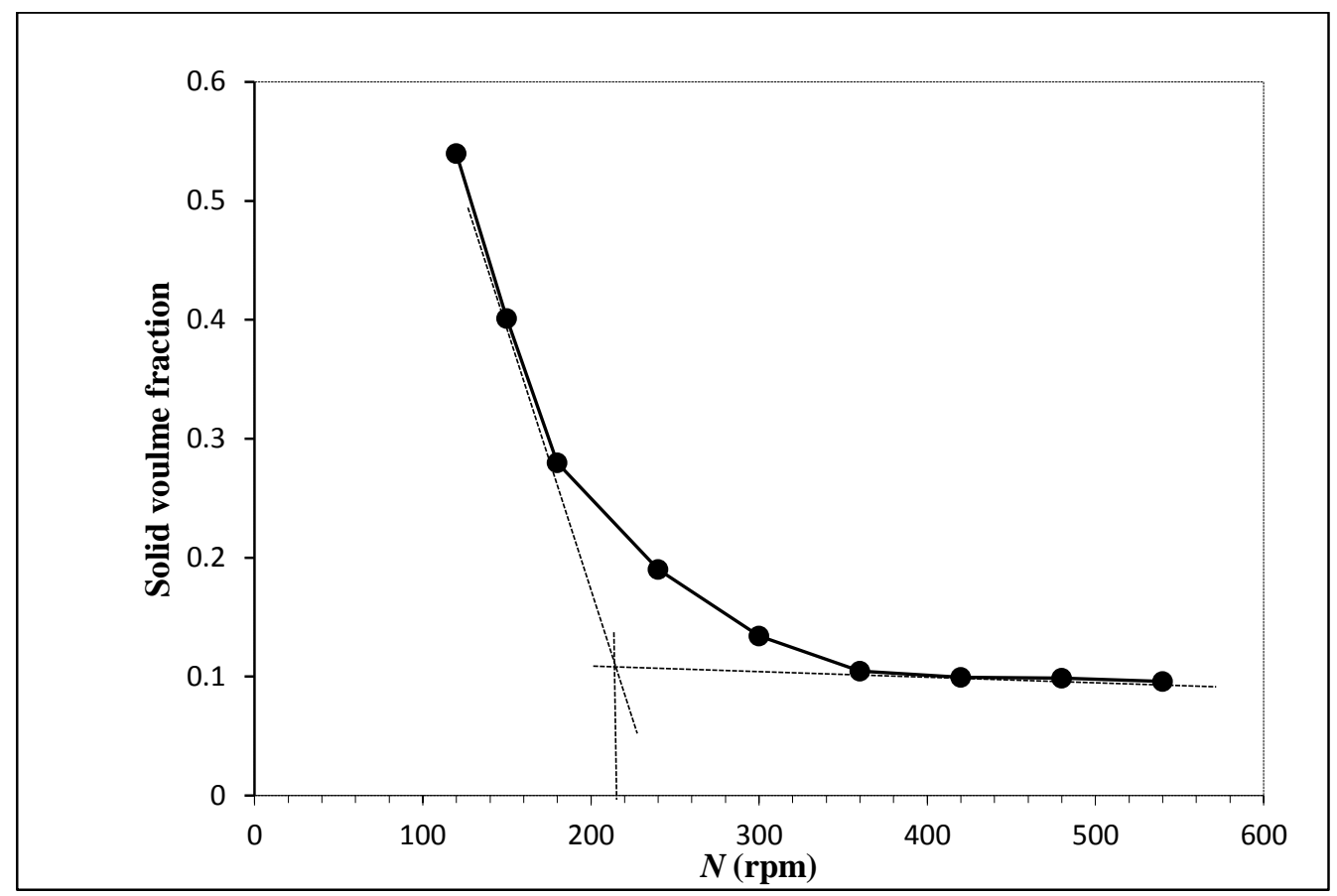

Figure (5.2-3). Estimation of just suspension speed using averaged solid volume fraction versus agitation speed for the Maxblend impeller $\left(d_{p}=209 \mu \mathrm{m}, X=10 \mathrm{wt} \%\right.$, and $\left.C=T / 8\right)$.

\subsubsection{Influence of Agitation Speed}

The CFD simulation was utilized to obtain the distributions of solid particles for four horizontal planes in order to estimate the extent of homogeneity. It should be noted that these planes were at the same location as those for the tomography planes. The data obtained from the CFD modeling was utilized to calculate the level of homogeneity inside the stirred tank. The extent of homogeneity was calculated using Equation (3.4-4). Figure (5.2-4) illustrates the impact of the stirrer speed on the extent of homogeneity for the Maxblend impeller. The CFD results, in terms of the level of homogeneity, are consistent with those retrieved from the ERT measurements. The standard deviation value between computed and measured homogeneity was found to be less than 2.7\%. As can be seen in Figure (5.2-4), the stirrer speed had significant impact on the level of homogeneity for the Maxblend impeller. The level of homogeneity in the mixing tank was improved with an increment in the stirrer speed pointing out the uniform suspensions. However, after attaining the maximum level of homogeneity, further raise in the stirrer speed had an adverse effect on the extent of homogeneity. Similar observations have also been reported in other research 
works (Hosseini et al., 2010a, 2010b; Tahvildarian et al., 2011). Hence, in order to achieve an enhanced mixing in the agitated tank, the determination of the optimum stirrer speed is crucial in solid-liquid mixing operations.

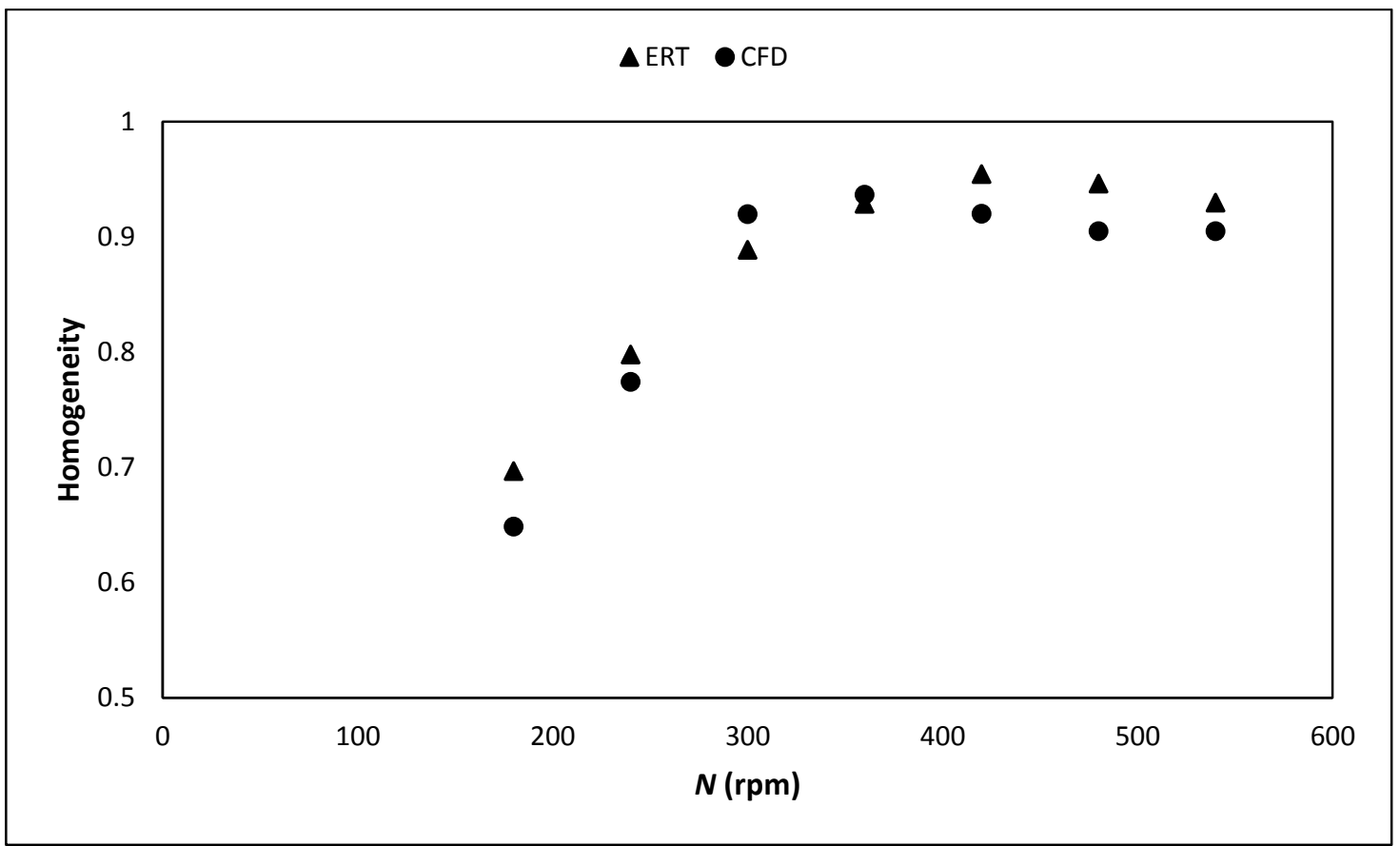

Figure (5.2-4). Influence of agitation speed on extent of homogeneity for the Maxblend impeller $\left(d_{p}=209 \mu \mathrm{m}, X=10 \mathrm{wt} \%\right.$, and $\left.C=T / 8\right)$.

\subsubsection{Particle Concentration Contours}

The CFD model was utilized to create particle concentration contours at different agitation speeds. Figure (5.2-5) illustrates the particle concentration contours at various stirrer speeds for the Maxblend impeller. As can be evident from Figure (5.2-5), the solid particles were accumulated at the base of the mixing vessel at the lower agitation speeds indicating that the particles were not completely suspended. The solid particles got uniformly suspended at the stirrer speed of $420 \mathrm{rpm}$. As the agitation speed increased to $480 \mathrm{rpm}$, the zones with low particle concentrations were created, which ultimately reduced the homogeneity level in the stirred vessel. In order to understand the impact of the agitation speed on the mixing quality in the stirred vessel precisely, particle concentration contours were generated at four different horizontal planes using the CFD 
model (Figure 5.2-6). At the stirrer speed of $N=180 \mathrm{rpm}$, the solid particles were accumulated at the lower region of the vessel. When the stirrer speed increased to $240 \mathrm{rpm}$, the solid particles reached to the top planes but they were not uniformly distributed. At the stirrer speed of $N=360$ rpm, the solid particles got uniformly distributed pointing out the enhanced mixing in the mixing vessel. Streamlines of the solid particles at the impeller speed of $N=420 \mathrm{rpm}$, confirmed the presence of the particles at the top of the mixing tank (Figure 5.2-7).

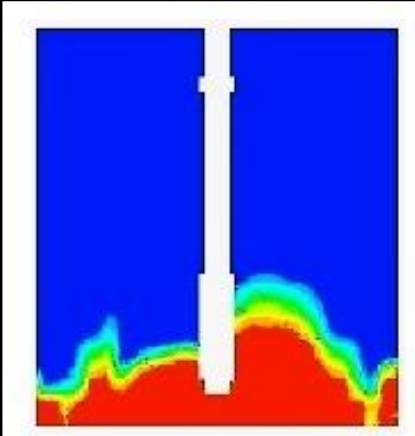

(a)

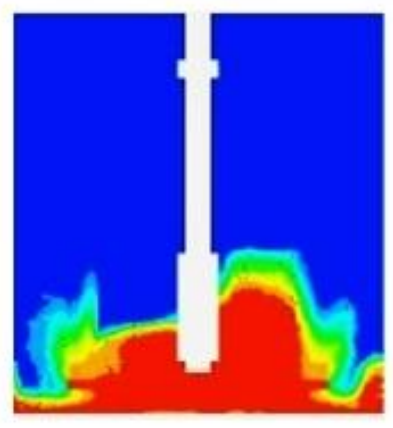

(b)

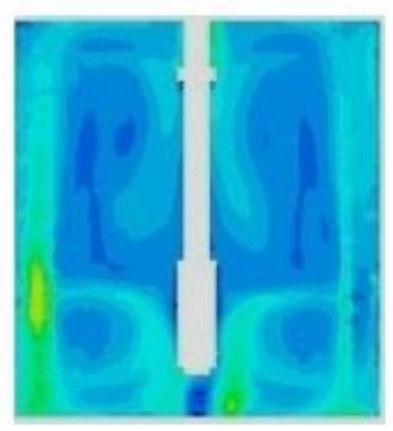

(c)

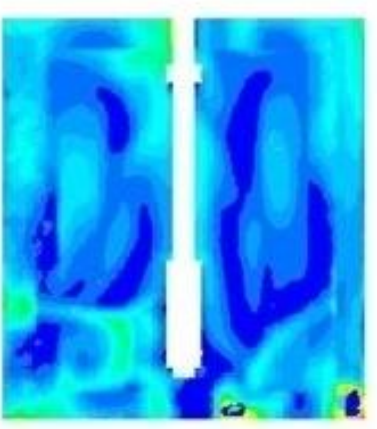

(d)

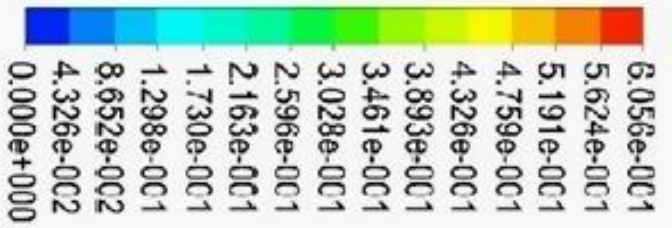

Figure (5.2-5). Particle concentration contours created by CFD at various agitation speeds for the Maxblend impeller ( $\left.d_{p}=209 \mu \mathrm{m}, X=10 \mathrm{wt} \%, C=T / 8\right)$ : (a) $N=120$, (b) $N=150$. (c) $N=420$, and (d) $N=480 \mathrm{rpm}$. 


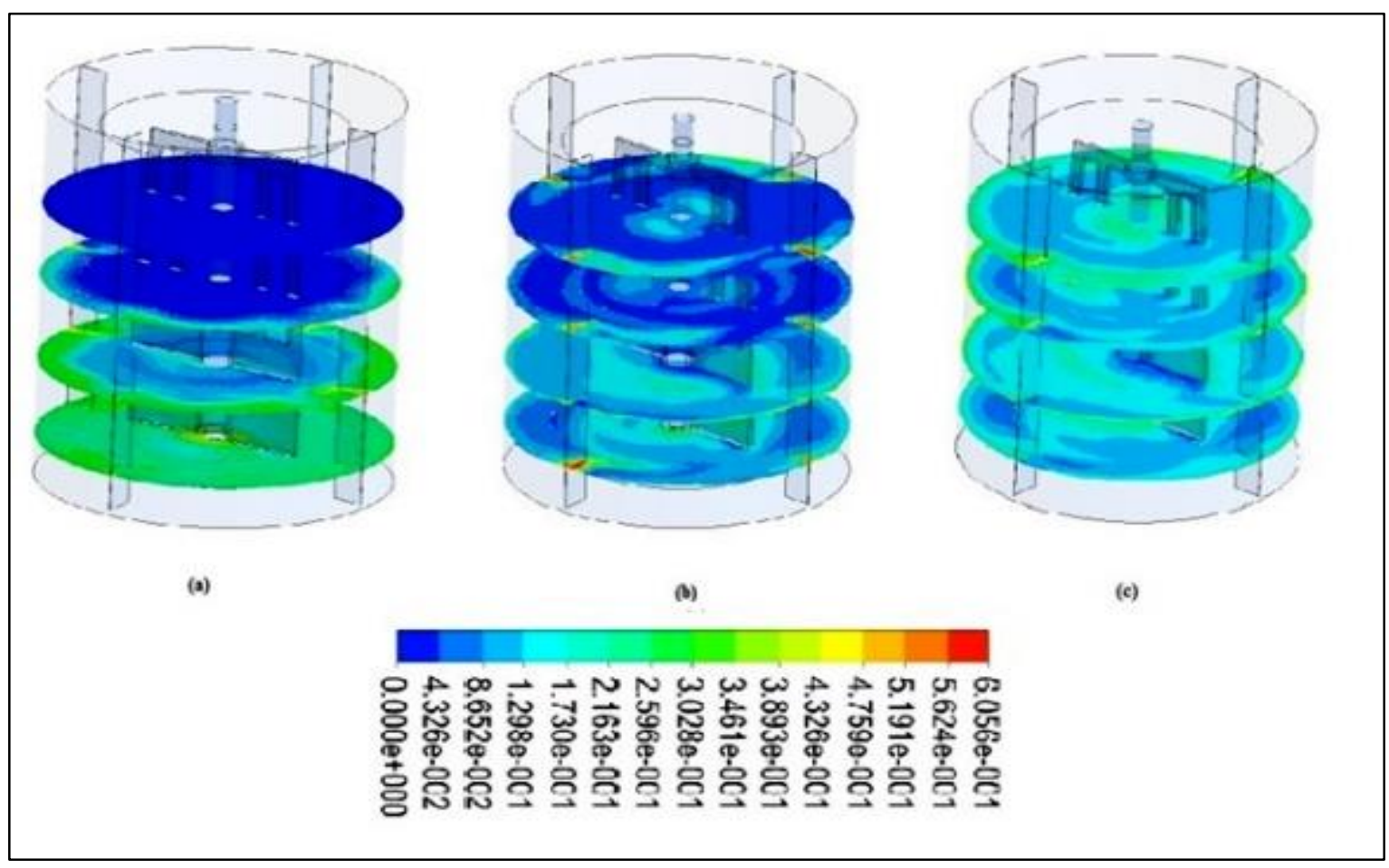

Figure (5.2-6). Employing CFD to create particle concentration contours for four different horizontal planes at various agitation speeds $\left(d_{p}=209 \mu \mathrm{m}, X=10 \mathrm{wt} \%\right.$, and $\left.C=T / 8\right)$ : (a) $N=$ 180, (b) $N=240$, and (c) $N=360 \mathrm{rpm}$.

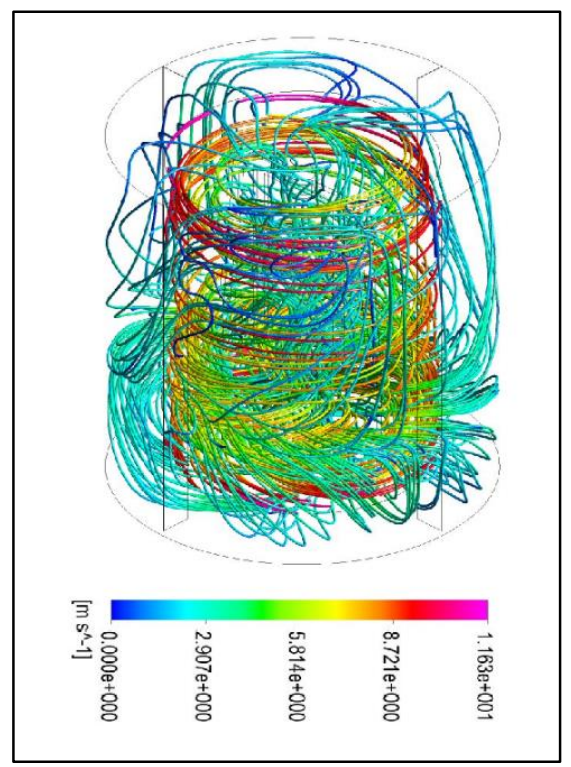

Figure (5.2-7). Streamlines of the solid particles for the Maxblend impeller $\left(\left(d_{p}=209 \mu \mathrm{m}, C=\right.\right.$ $T / 8, X=10 \mathrm{wt} \%$, and $N=420 \mathrm{rpm}$ ). 


\subsubsection{Axial Particle Concentration Profiles}

The validated model was utilized to create axial particle concentration profiles at various agitation speeds to quantify the solid particles distribution in the stirred vessel for the Maxblend impeller. The solid volume fractions $\left(X_{v}\right)$ computed for each horizontal plane was divided by the averaged solid volume fraction $\left(\bar{X}_{v}\right)$ within the vessel to obtain the normalized solid volume fraction. Figure (5.2-8) illustrates the axial concentration profiles at different agitation speeds for the Maxblend impeller. Homogeneity of the system increased as $X_{v}$ approaches $\bar{X}_{v}$. When $X_{v}=$ $\bar{X}_{v}$, the mixing in the stirred vessel resulted in the perfect mixing condition (homogeneity $=1$ ).

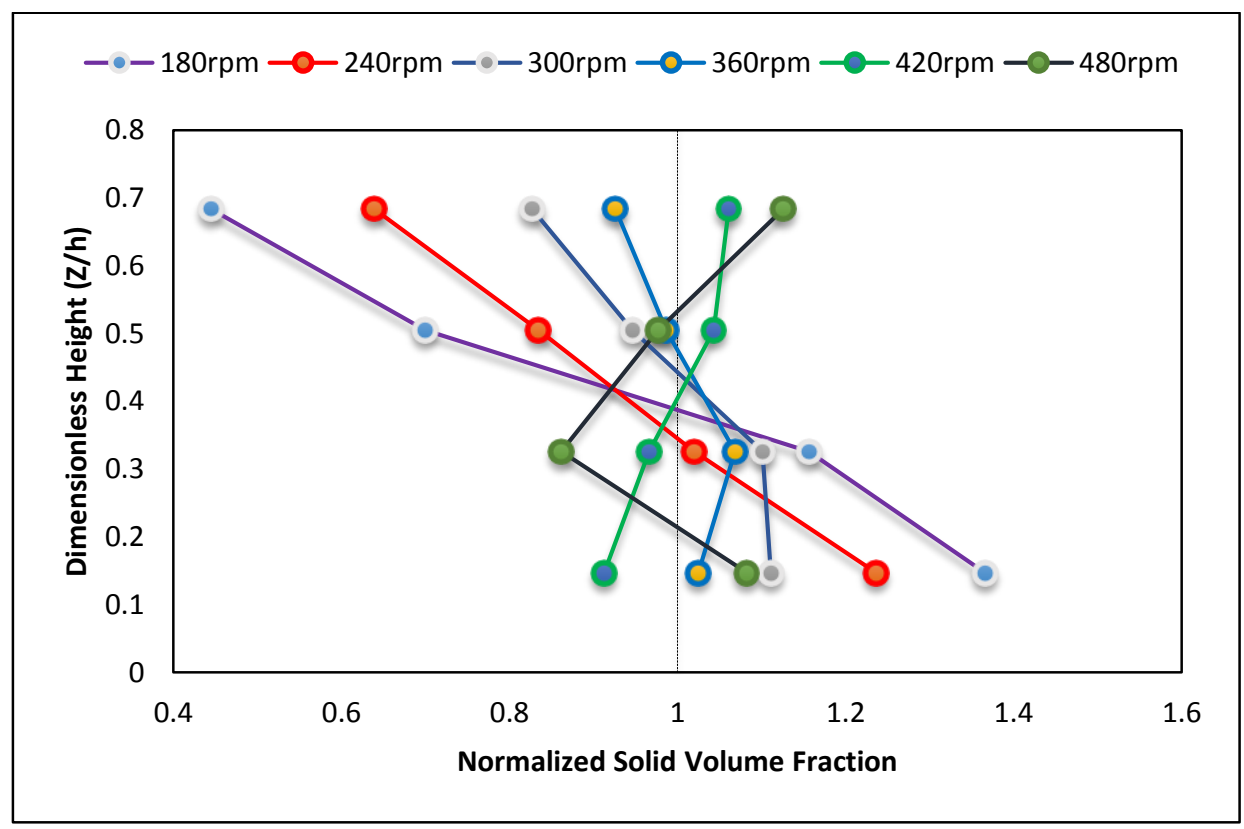

Figure (5.2-8). Axial particle concentration profile calculated using CFD for the Maxblend impeller $\left(d_{p}=209 \mu \mathrm{m}, X=10 \mathrm{wt} \%\right.$, and $\left.C=T / 8\right)$.

\subsubsection{Clouding Height}

Several researchers have assessed the degree of particle suspensions in terms of clouding height. A distinct solid-liquid interface exists above which the solid particle concentration is almost nil for high particles loadings. The height (distance) of the clear interface from the base of the stirred tank is referred to as the clouding height (Bittorf and Kresta, 2003). This distinct interface distinguishes the clear liquid and solid suspension zones. In this work, clouding height 
was estimated by computing the particle concentration contours on a vertical plane using the validated CFD model. Figure (5.2-9) shows the clouding heights at $N=150$ and $420 \mathrm{rpm}$. The effect of the stirrer speed on the normalized clouding height was examined and analysed for the Maxblend impeller (Figure 5.2-10). As can be observed in Figure (5.2-10), the normalized clouding height increased with an increment in the agitation speed indicating the increase of the suspension region. The clear liquid region vanished when the normalized clouding height reached the maximum value of 1. Figure (5.2-9) shows that the clear liquid region disappeared at the agitation speed of $420 \mathrm{rpm}$. Similar observations have been reported in numerous research works for other impellers (Hicks et al., 1996; Micale et al., 2004; Bittorf and Kresta, 2003).

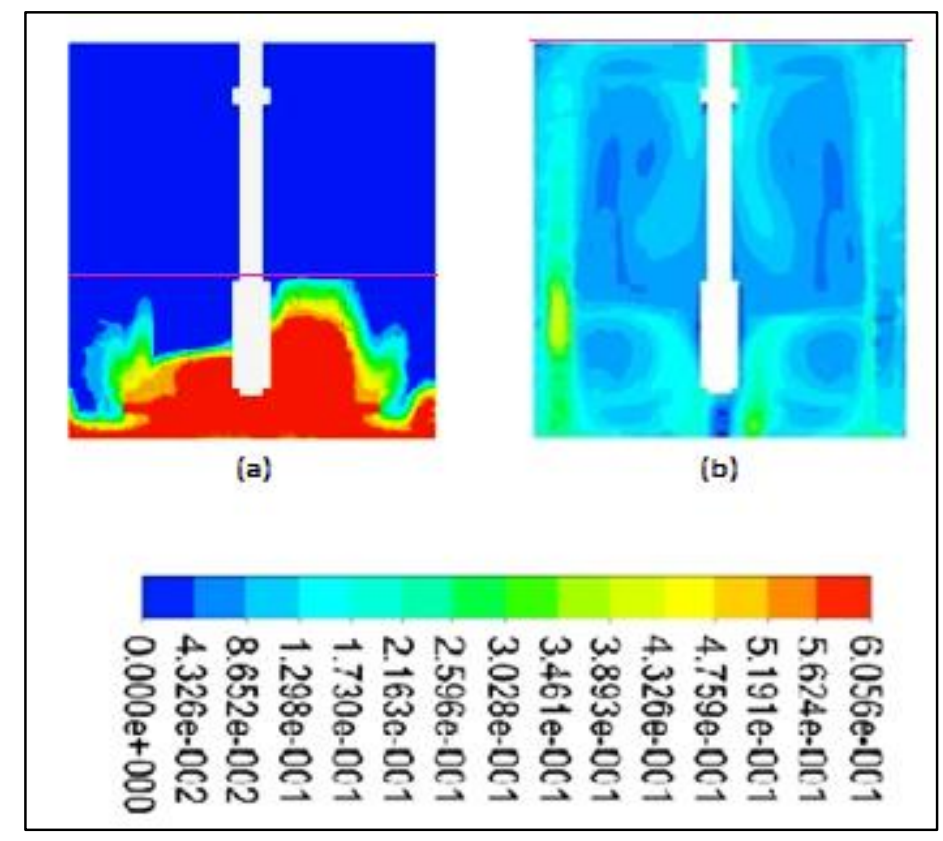

Figure (5.2-9). Clouding height for the Maxblend impeller measured ( $d_{p}=209 \mu \mathrm{m}, X=10 \mathrm{wt} \%$, $C=T / 8$ ): (a) $N=150$, and (b) $N=420 \mathrm{rpm}$. 


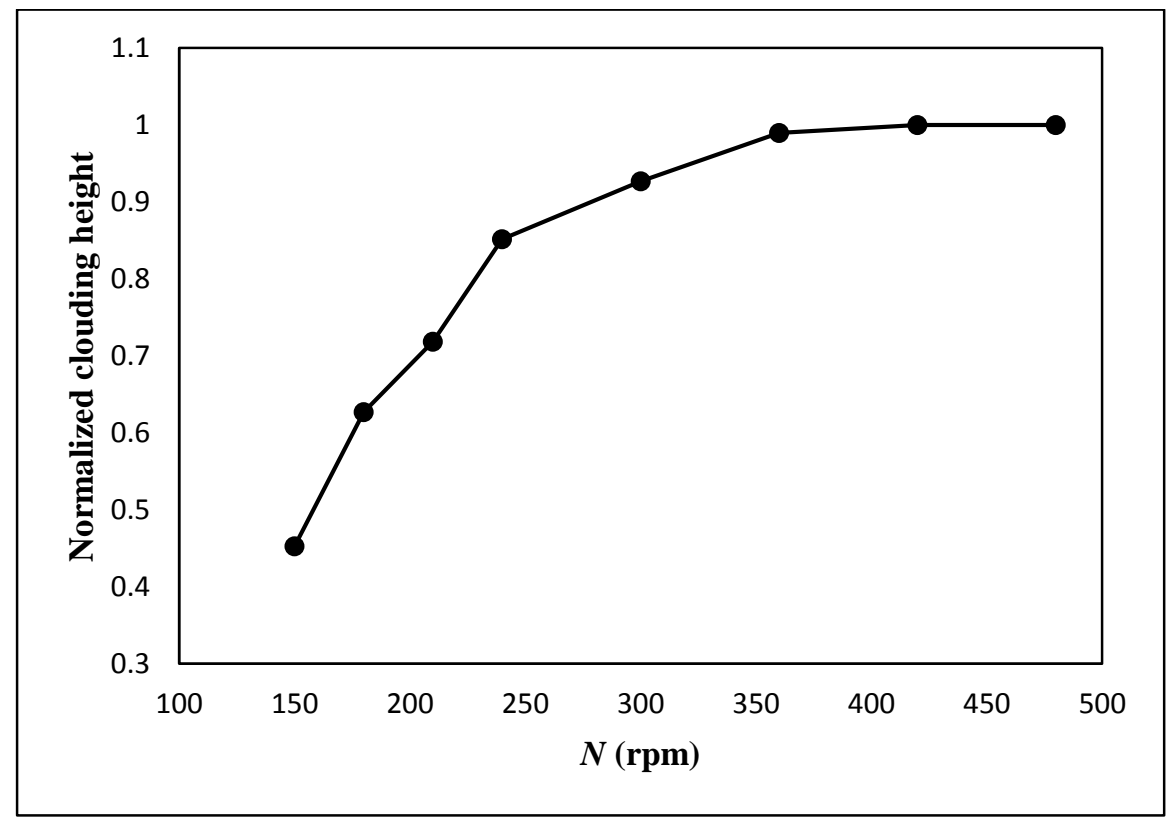

Figure (5.2-10). Normalized clouding height at different agitation speeds for the Maxblend impeller $\left(d_{p}=209 \mu \mathrm{m}, X=10 \mathrm{wt} \%\right.$, and $\left.C=T / 8\right)$.

\subsubsection{Impact of Baffles}

The Maxblend impellers can be utilized in mixing operations with and without baffles. In this work, the influence of baffles on the solid suspensions was examined using the validated CFD model. Figure (5.2-11) depicts the impact of baffles on the extent of homogeneity for the Maxblend impeller at various impeller speeds for the solid-liquid mixing operation. As can be seen in Figure (5.2-11), the extent of homogeneity increased with the raise in the agitation speed for both unbaffled and baffled mixing tanks. The peak level of homogeneity achieved for the unbaffled tank was significantly lower than that for the baffled vessel at the same agitation speed. It should be noted that the extent of homogeneity reduced beyond the optimal agitation speed in an unbaffled tank. The particles were not dispersed uniformly throughout the unbaffled stirred vessel due to the formation of the vortex and dead zones at the higher stirrer speeds (Figure 5.2-12). Baffles are essentially fixed to avoid the creation of vortex and dead zones, and to control the fluid flow inside the stirred vessel (Paul et al., 2004). It can be concluded that the use of baffles improved the solid suspensions inside the stirred tank equipped by a Maxblend impeller. 


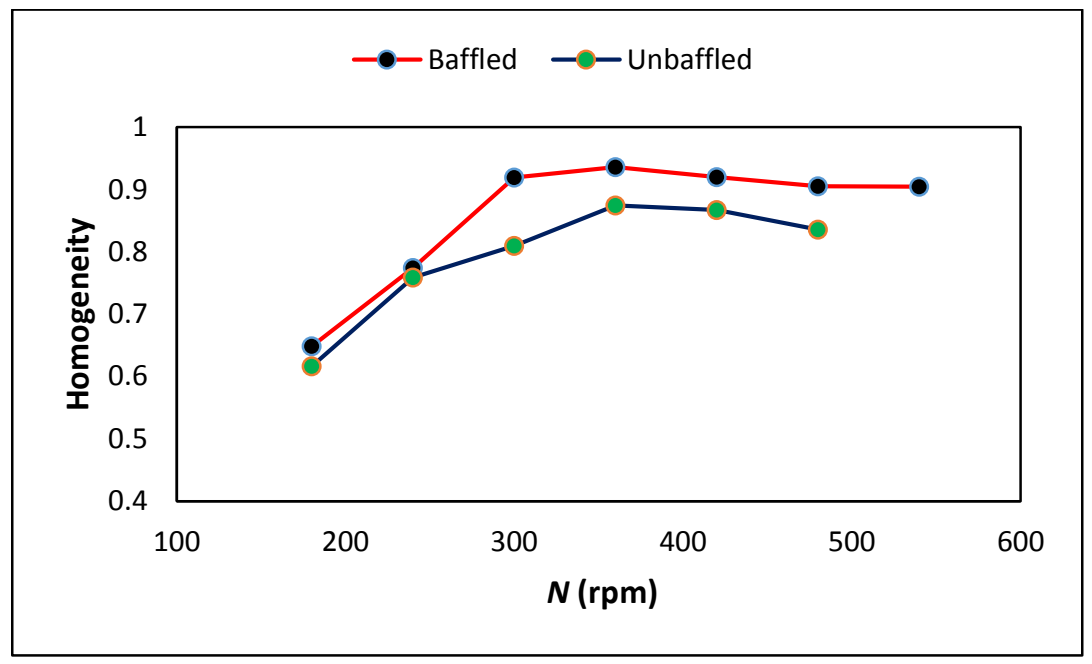

Figure (5.2-11). Influence of baffles on level of homogeneity at different agitation speeds for the Maxblend impeller $\left(d_{p}=209 \mu \mathrm{m}, X=10 \mathrm{wt} \%\right.$, and $\left.C=T / 8\right)$.

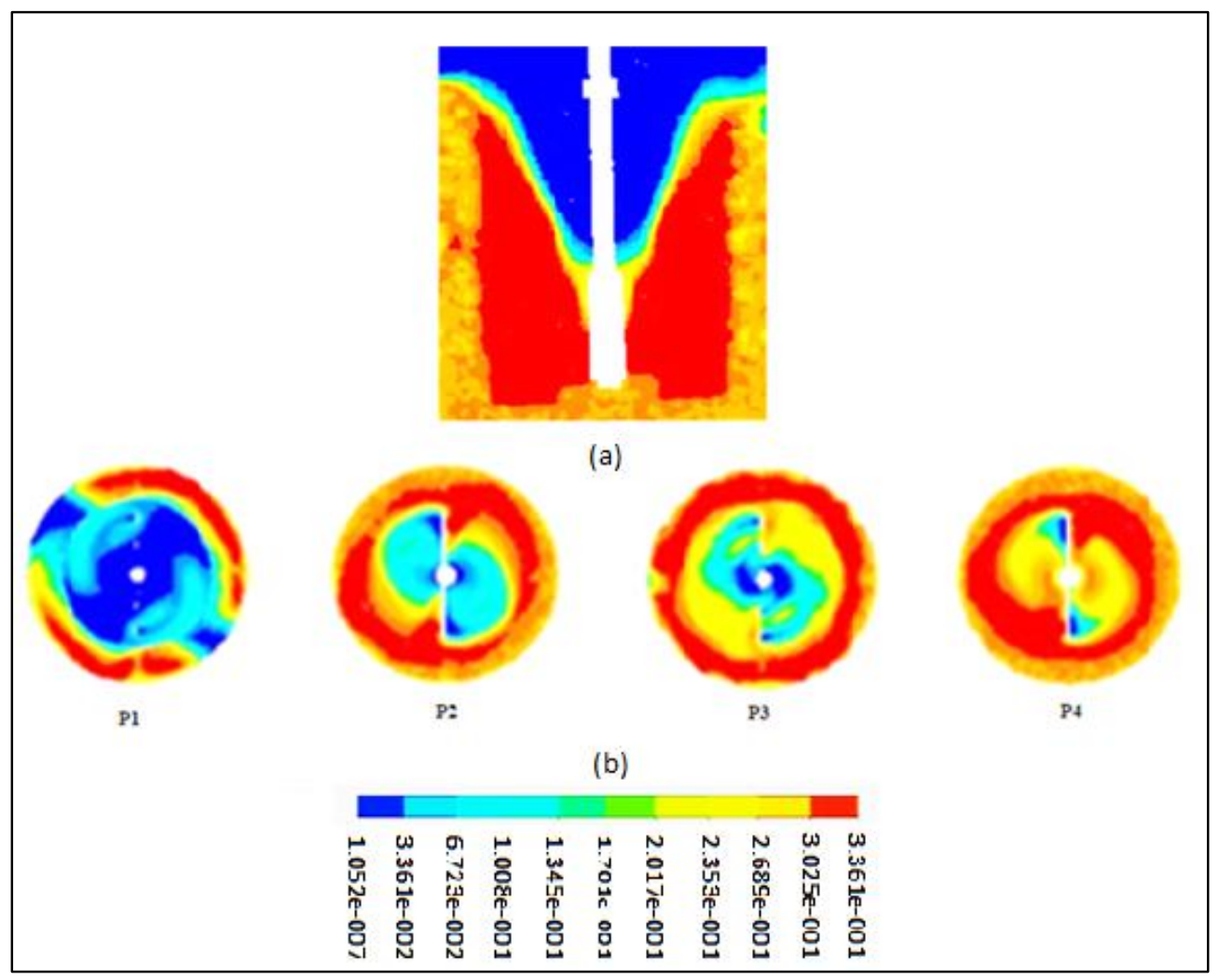

Figure (5.2-12). (a) Particle concentration contours at a vertical plane, and (b) particle concentration contours at different horizontal planes for the Maxblend impeller without baffles $\left(d_{p}\right.$ $=209 \mu \mathrm{m}, X=30 \mathrm{wt} \%, C=T / 8$, and $N=420 \mathrm{rpm})$. 


\subsubsection{Mixing Index}

Some researchers utilized the axial concentration profiles to determine the extent of suspension for the liquid-solid mixing operations. However, the effect of the radial concentration gradients on the solid suspensions needs to be taken into considerations as well to understand the local mixing quality precisely inside the mixing tank (Michelletti et al., 2003). The validated CFD model was employed to generate velocity vectors to understand the flow pattern inside the mixing vessel furnished with the Maxblend impeller. As can be evident from Figure (5.2-13), the Maxblend impeller generated both axial and radial flow patterns. A new mixing index was proposed in this work to quantify the solid suspensions in the stirred vessel taking the effects of both radial and axial concentration gradients into account:

$$
\text { Mixing index }=\sqrt{\frac{\sum_{1}^{c}\left(X_{v c}-\overline{X_{v}}\right)^{2}}{c-1}}
$$

where $X_{v c}, \overline{X_{v}}$, and $c$ are the solid volume fraction at each point location, averaged solid volume fraction, and the number of contour points, respectively. The CFD model was utilized to obtain four vertical planes at spacing of $90^{\circ}$. Then, 20 point locations were selected in each vertical plane. The total number of contour points selected in this study was 80. Similar approach was adopted by Carletti et al. (2014) to quantify the solid distributions in the mixing vessel equipped with PBT impellers using ERT. However, Carletti et al. (2014) selected mean conductivity values at 1264 pixels instead of solid volume fraction to develop the mixing index. Figure (5.2-14) illustrates the influence of the stirrer speed on the mixing index defined by Equation (5.2-1). It can be noted that the mixing index decreased with the increase of the agitation speed indicating the enhanced mixing in the mixing vessel. However, the mixing quality reduced with further increment in the stirrer speed beyond the optimum value. It is essential to mention that the mixing index increased at the impeller speed higher than the optimal impeller speed of $420 \mathrm{rpm}$. This result is in agreement with the results presented in Figure (5.2-4). Hence, it can be concluded that the effects of radial concentration gradients are not significant for the Maxblend impeller. 


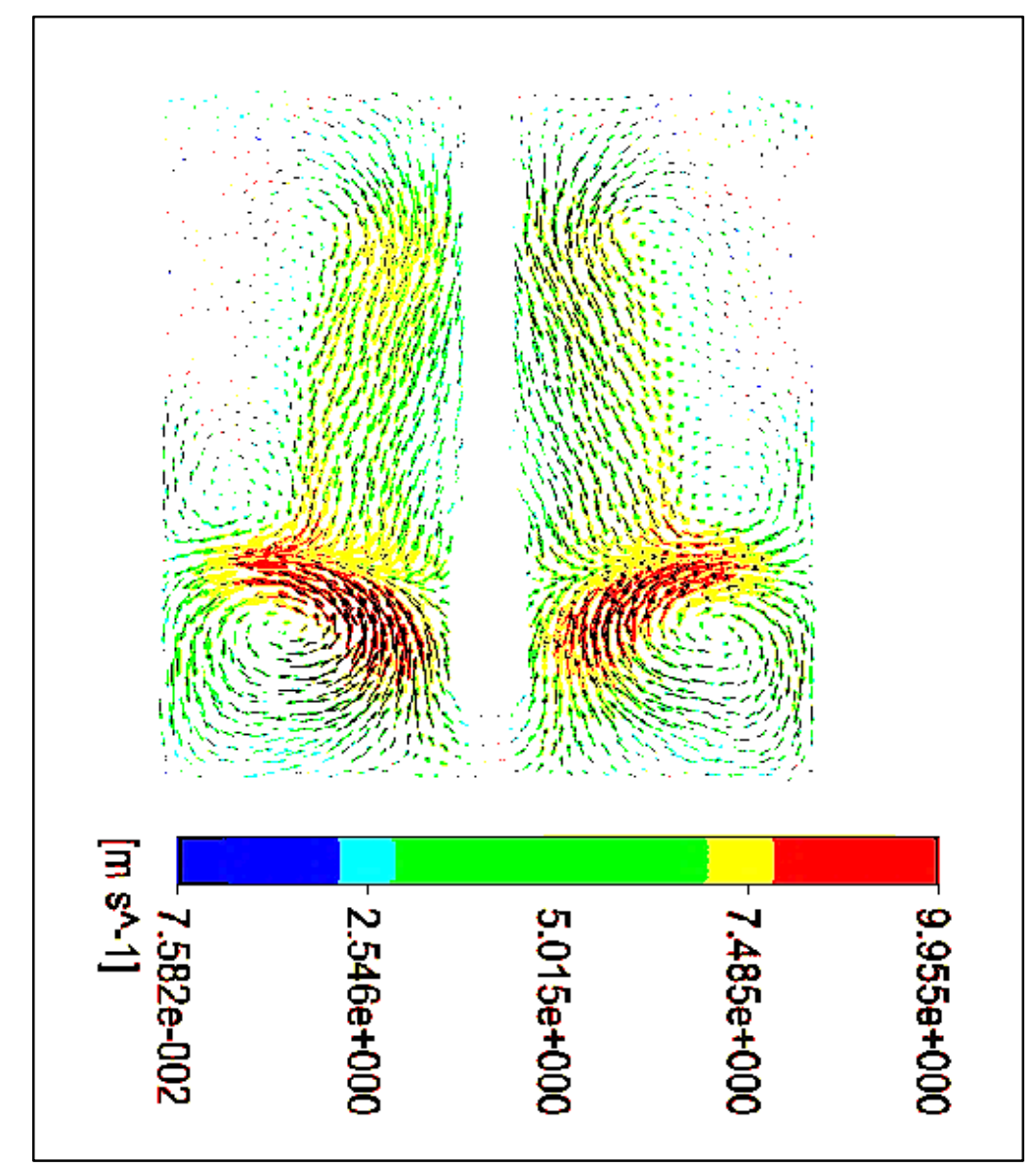

Figure (5.2-13). Velocity vectors for solid particles for the Maxblend impeller $\left(d_{p}=209 \mu \mathrm{m}, X=\right.$ $10 \mathrm{wt} \%, C=T / 8$, and $N=480 \mathrm{rpm})$.

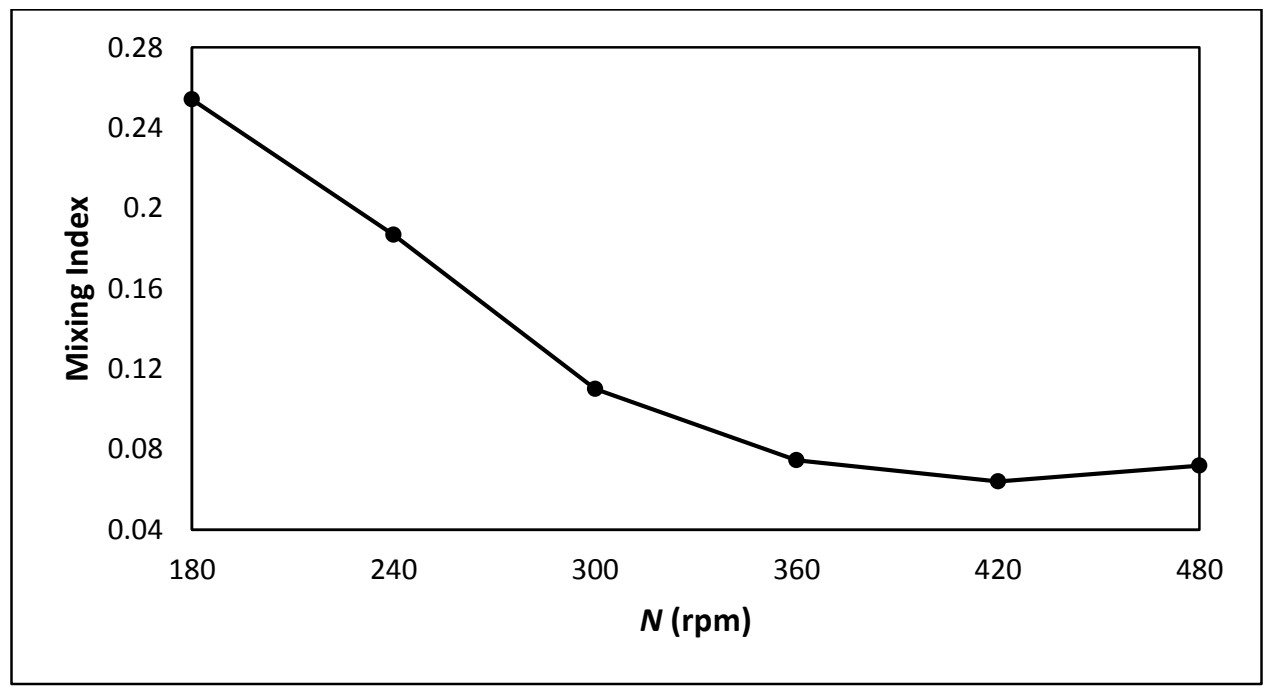

Figure (5.2-14). Impact of agitation speed on mixing index for the Maxblend impeller $\left(d_{p}=209\right.$ $\mu \mathrm{m}, C=T / 8$, and $X=10 \mathrm{wt} \%)$. 


\subsubsection{Effect of Particle Diameter}

Physical characteristics of the particles particularly the average size of the solid particles significantly impact the mixing quality in the solid suspensions. The impact of the average particle size (diameter) on the extent of homogeneity was analyzed in this paper. As can be observed in Figure (5.2-15), the level of homogeneity increased with a decrease in the particle diameter. Figure (5.2-16) demonstrates that the solid particles $(111 \mu \mathrm{m})$ were distributed uniformly inside the mixing vessel. However, the solid suspension was not uniform for the particles with the average size of size $752 \mu \mathrm{m}$. Similar observations have been reported in previous research works (Godfrey and Zhu, 1994; Hosseini et al., 2010a, 2010b; Tahvildarian et al., 2011). The following equation developed by Perry and Green (1984) could shed light on the effect of particle diameter on solidliquid suspensions:

$$
V_{t}=\left[\frac{4 g_{c} d_{p}\left(\rho_{p}-\rho_{l}\right)}{3 C_{D} \rho_{l}}\right]^{1 / 2}
$$

where $V_{t}, C_{D}, \rho_{p}, \rho_{l}, d_{p}$, and $g_{c}$ are particle terminal settling velocity, the drag coefficient, density of solid particle, liquid density, diameter of particle and gravitational constant, respectively. A decrement in average particle size reduces the particle terminal settling velocity (Eq. 5.2-2). Hence, settling of the finer solid particles would be slower in comparison to that for the larger ones indicating that the uniform distribution of the large solid particles would not be easier. 


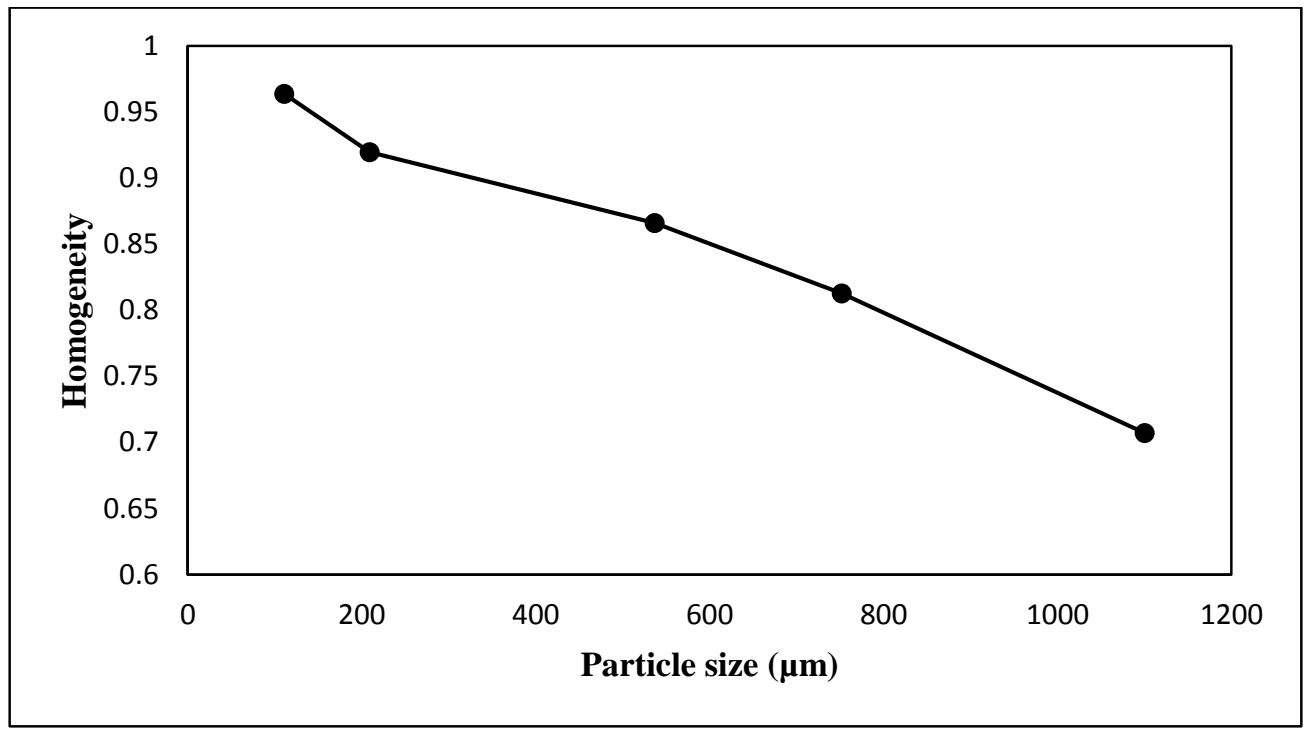

Figure (5.2-15). Influence of the solid particle diameter on the extent of homogeneity for the Maxblend impeller $(C=T / 8, X=10 \mathrm{wt} \%$, and $N=300 \mathrm{rpm})$.

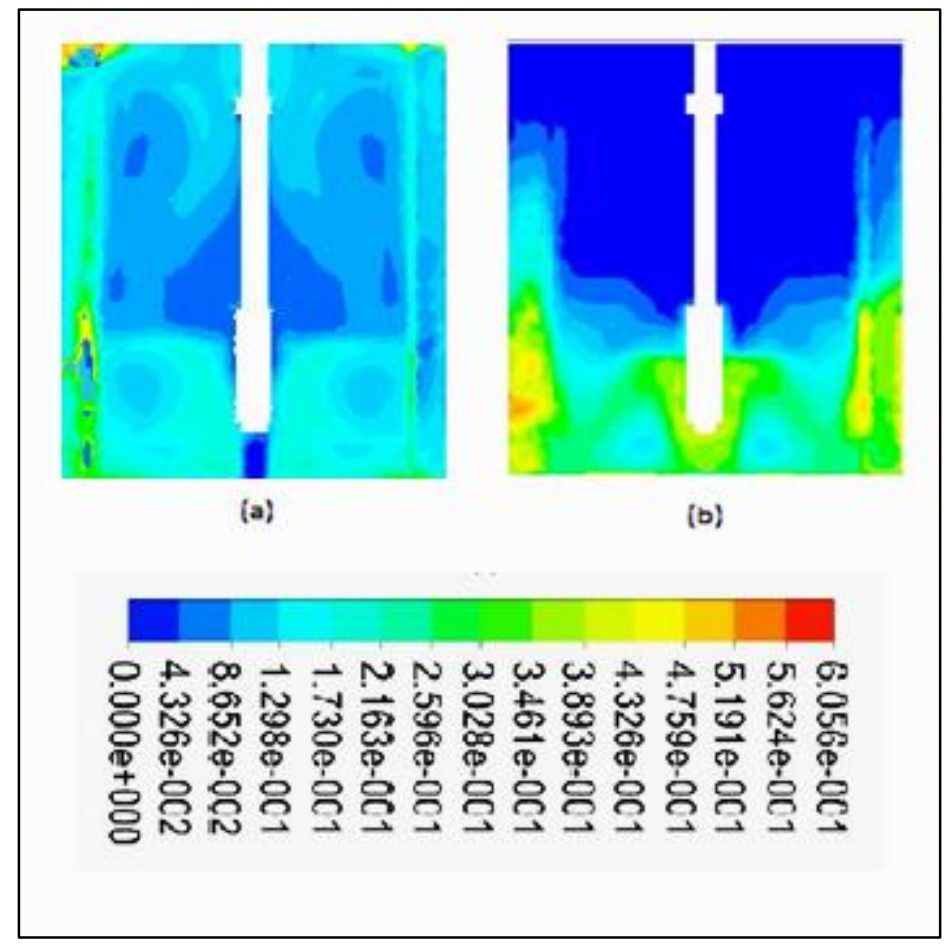

Figure (5.2-16). Particle concentration contours for the Maxblend impeller $(C=T / 8, X=10 \mathrm{wt} \%$, and $N=300 \mathrm{rpm}$ ): (a) $d_{p}=111$, and (b) $d_{p}=752 \mu \mathrm{m}$. 


\subsubsection{Effect of Particle Specific Gravity}

The solid-liquid suspension is greatly influenced by the particle specific gravity (SG). The developed CFD modeling was employed to assess the effect of the particle specific gravity (SG) on the extent of homogeneity for the Maxblend impeller. As depicted in Figure (5.2-17), the level of homogeneity reduced with an increase in the specific gravity of the solid particles. The particles with the specific gravity of 5 were accumulated at the base of the tank whereas the particles with the specific gravity of 1.5 were uniformly suspended at the rotational speed of $300 \mathrm{rpm}$ (Figure 5.2-18). The solid particles with a higher specific gravity show more resistance to the fluid flow, which eventually reduced the level of homogeneity. Similar phenomenon was observed by Hosseini et al. (2010b) for the A310 impeller. It can be concluded that the particle specific gravity has pronounced influence on the local mixing quality in the stirred tank.

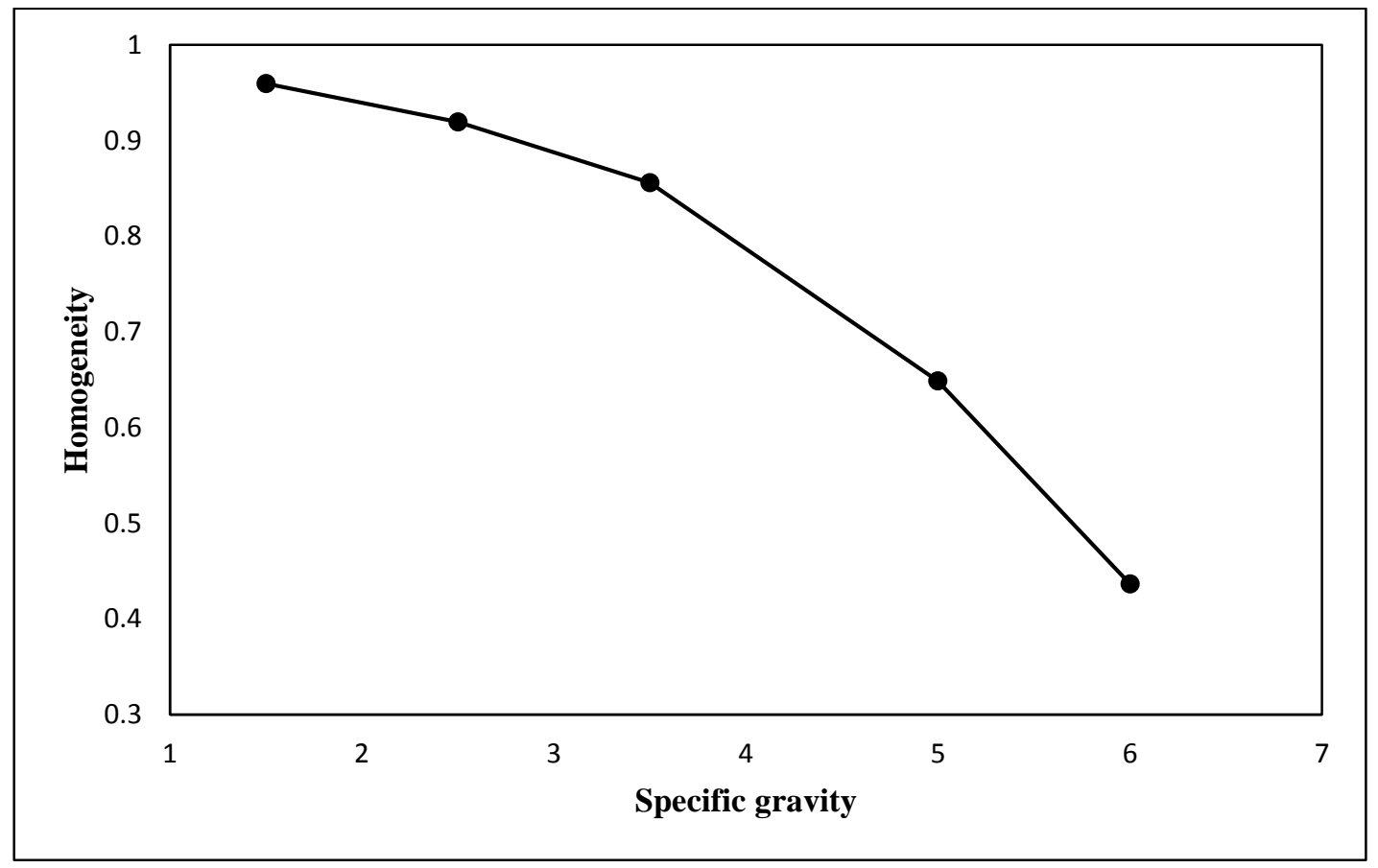

Figure (5.2-17). Extent of homogeneity versus particle specific gravity for the Maxblend impeller ( $d_{p}=209 \mu \mathrm{m}, C=T / 8, X=10 \mathrm{wt} \%$, and $N=300 \mathrm{rpm}$ ). 


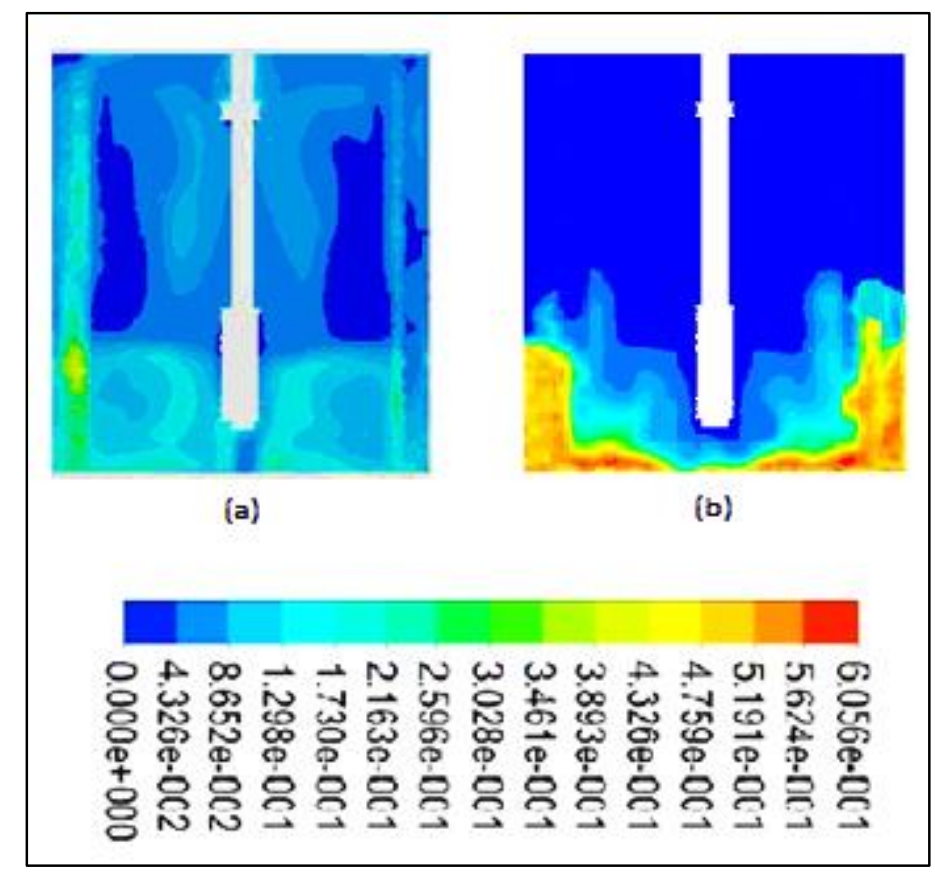

Figure (5.2-18). Particle concentration contours for the Maxblend impeller $\left(d_{p}=209 \mu \mathrm{m}, C=\right.$ $T / 8, X=10 \mathrm{wt} \%$, and $N=300 \mathrm{rpm}$ ): (a) $\mathrm{SG}=1.5$, and (b) $\mathrm{SG}=5$.

\subsubsection{Effect of Particle Concentration}

The solid particle loading has a significant influence on the particle suspension in the mixing vessel. The impact of the solid loading on the level of homogeneity was examined using the developed CFD modeling. The solid particle loading was varied from 5 to $30 \mathrm{wt} \%$ in this study. Figure (5.2-19) demonstrates the influence of the solid particle loading on the level of homogeneity at $N=480 \mathrm{rpm}$. The results clearly show that the highest homogeneity level was obtained at the highest solid particle loading of $30 \mathrm{wt} \%$. These CFD results are consistent with the results mentioned by some researchers (Godfrey and Zhu, 1994; Hosseini et al., 2010a). In fact, the density and viscosity of the slurry increase with an increase in the solid particle loading. At the higher solid loadings, the terminal settling velocity of the solid particles changes to the hindered settling velocity because of the increment in the density and viscosity of the slurry, interactions among solid particles and the contact of falling particles with the upward flowing fluid (Paul et al., 2004). The following correlation developed by Maude and Whitmore (1958) quantifies the relationship between the free solid particle settling velocity and the hindered settling velocity in terms of the solid volume fraction: 


$$
V_{t s}=V_{t}\left(1-X_{v}\right)^{n_{t}}
$$

where $V_{t}$ and $V_{t s}$ are terminal settling velocity and hindered settling velocity of particles, respectively. $n_{t}=2.33$ for $R e_{P}>1000, n_{t}=4.375 R e_{P}^{-0.0875}$ for $0.3<R e_{P} \leq 1000$ and $n_{t}=$ 4.64 for $R e_{P} \leq 0.3$, where $R e_{P}$ represents the particle Reynolds number. $X_{v}$ is the solid volume fraction. The extent of homogeneity was improved at the higher particle loadings due to the effect of the hindered settling velocity. The uniform distribution of the particles increased with the increment in the solid loadings resulting in the improved mixing quality.

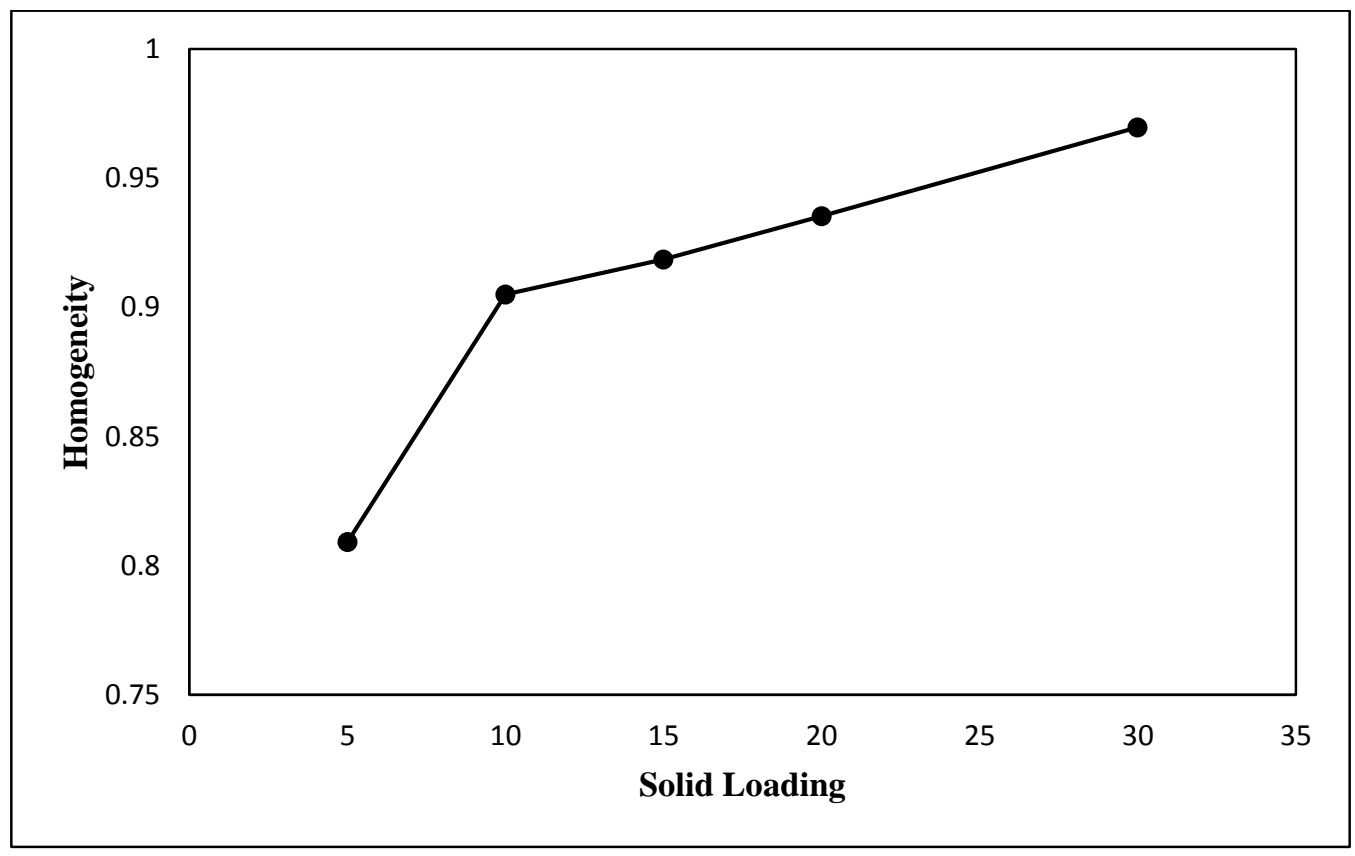

Figure (5.2-19). Influence of the solid loadings on the extent of homogeneity for the Maxblend impeller $\left(d_{p}=209 \mu \mathrm{m}, C=T / 8\right.$, and $\left.N=480 \mathrm{rpm}\right)$. 


\section{Chapter 6}

\section{CONCLUSIONS}

The mixing performance of the Maxblend impeller in a slurry reactor was successfully investigated and analyzed using ERT and CFD techniques. The 2D and 3D tomography images were employed to assess the particles distribution inside the mixing vessel. The developed CFD model provided detailed insight into the particle concentration and distribution within the stirred tank. The generated CFD model was validated and verified by comparing the results obtained from the simulation to the experimentally determined values in terms of the stirrer torque, just suspension speed, and extent of homogeneity. The results obtained from the CFD modeling were consistent with those attained from the ERT measurements. The particle concentration profiles were utilized to evaluate the extent of homogeneity and mixing index for the solid-liquid mixing system. To assess the mixing efficiency and effectiveness of the Maxblend impeller precisely, different mixing indices were utilized in this study. The ERT and CFD results showed that the extent of homogeneity and mixing indices were enhanced with an increment in the agitation speed. However, after attaining the peak homogeneity level, further increment in the agitation speed had an adverse impact on the extent of homogeneity and mixing indices. The results also demonstrated that the maximum attainable homogeneity for the Maxblend impeller was enhanced with an increase in the solid loading due to the impact of the hindered settling velocity. It should be noted that the use of baffles improved the mixing quality achieved by the Maxblend impeller with a lower power consumption. The ERT results clearly showed that the highest homogeneity level was attained for the smallest solid particles (209 microns). Experimental tests demonstrated that maximum homogeneity attained by the Maxblend impeller was higher than those for the A200 and Rushton impellers. It was also found that the highest level of homogeneity was obtained for the impeller clearance of $C=T / 8$. The particle distribution inside the mixing tank was also quantified using the clouding height approach. The effect of the radial concentration gradients was analyzed using a new mixing index for the slurry reactor. The results obtained from the CFD simulation 
clearly demonstrated that the physical characteristics of the particle such as the specific gravity and the particle size had pronounced influence on the extent of homogeneity in a slurry reactor. Overall, the Maxblend impeller demonstrated an excellent performance in the solid-liquid mixing operations. Employing the findings of this investigation can lead to an efficient and effective usage of power, improved quality of products, enhanced production rate, and the operating cost reduction for the slurry reactors. 


\subsection{Recommendations for Future Research Work}

After thorough experimental tests and CFD simulations, the following suggestions are offered for further research work with respect to multi-phase flow and mixing behaviour:

- Solid-liquid mixing behaviour in continuous-flow mixers should be investigated.

- The mixing performance of the Maxblend impeller for the multi-phase mixing operations (e.g. liquid-liquid, gas-liquid) should be explored.

- To understand the effect of fluid rheology, multiphase mixing in non-Newtonian fluid medium should be considered.

- To study the particle-particle interaction precisely, CFD-DEM coupling should be employed for the simulation. 


\section{Nomenclature}

A

B

Ar

a

$B_{o}$

C

c

$C_{D}$

$C_{H}$

$C_{s}$

$C_{i j}$

$C_{M}$

$C_{v}$

$C_{1 \varepsilon}, C_{2 \varepsilon}, C_{3 \varepsilon}$

$C_{\mu}$

$c_{c}$

$c_{p}$

D

$D_{e, P}$

$d_{p}$

$\bar{E}_{s t}$

F

$\boldsymbol{F}_{a c}$

$\vec{F}_{B}, F_{B}$

$\boldsymbol{F}_{B H}$

$F_{c}$

$\boldsymbol{F}_{c b}$

$\vec{F}_{D}, \boldsymbol{F}_{D}$ function of liquid volume fraction (Equation (2.8-21))

function of liquid volume fraction (Equation (2.8-22))

Archimedes number

acceleration, $\mathrm{m} / \mathrm{s}^{2}$

particle concentration percent

impeller off-bottom clearance, $\mathrm{m}$

total number of contour points

drag coefficient, dimensionless

clouding height, $\mathrm{m}$

Saffman lift force constant

local particle distribution at $\mathrm{i}^{\text {th }}$ position and $\mathrm{j}^{\text {th }}$ speed, dimensionless

mean particle distribution, dimensionless

volume fraction of the particle

empirical constants

eddy (turbulent) viscosity constant

velocity of sound in the liquid medium, $\mathrm{m} / \mathrm{s}$

velocity of sound in the particle, $\mathrm{m} / \mathrm{s}$

impeller (stirrer) diameter, $\mathrm{m}$

particle diffusion coefficient

solid particle diameter, $\mu \mathrm{m}$

energy density of the developed waves, $\mathrm{kgm}^{-1} \mathrm{~s}^{-2}$

force, $\mathrm{N}$

acoustic force, $\mathrm{N}$

buoyant force, $\mathrm{N}$

Basset force, $\mathrm{N}$

centrifugal force, $\mathrm{N}$

buoyant force under centrifugal field, $\mathrm{N}$

drag force, $\mathrm{N}$ 


\begin{tabular}{|c|c|}
\hline$\vec{F}_{G}, \boldsymbol{F}_{G}$ & gravitational force, $\mathrm{N}$ \\
\hline$\vec{F}_{L}, F_{L}$ & lift force, $\mathrm{N}$ \\
\hline $\boldsymbol{F}_{M}$ & Magnus lift force, $\mathrm{N}$ \\
\hline$F_{p}$ & force due to pressure gradient, $\mathrm{N}$ \\
\hline$F_{s}$ & shear force, $\mathrm{N}$ \\
\hline$F_{\text {saff }}$ & Saffman lift force, $\mathrm{N}$ \\
\hline$\vec{F}_{v m}, \boldsymbol{F}_{A}$ & virtual (added) mass force, $\mathrm{N}$ \\
\hline $\mathrm{Fr}^{*}$ & modified Froude number \\
\hline$f$ & drag function \\
\hline$g, g_{c}, \boldsymbol{g}, \vec{g}$ & gravitational acceleration, $\mathrm{m} / \mathrm{s}^{2}$ \\
\hline$G_{b}$ & turbulent kinetic energy due to buoyancy effect, $\mathrm{kg} / \mathrm{ms}^{3}$ \\
\hline$G_{k}$ & turbulent kinetic energy due to velocity gradients, $\mathrm{kg} / \mathrm{ms}^{3}$ \\
\hline$H$ & height of the mixing vessel, $\mathrm{m}$ \\
\hline$h$ & height of the liquid inside the mixing vessel, $\mathrm{m}$ \\
\hline$\overline{\bar{I}}$ & unit tensor \\
\hline$J_{\infty}$ & Saffman lift function \\
\hline$K$ & empirical constant \\
\hline$K_{l q}$ & exchange coefficient between fluid and $q^{\text {th }}$ phase \\
\hline$K_{p l}$ & exchange coefficient between solid and liquid phase \\
\hline$k$ & kinetic energy due to turbulence, $\mathrm{m}^{2} \mathrm{~s}^{2}$ \\
\hline$k_{a}$ & wave number, $\mathrm{m}^{-1}$ \\
\hline$L$ & length, $\mathrm{m}$ \\
\hline$m$ & mass, kg \\
\hline$m_{c}$ & mass of continuous phase, $\mathrm{kg}$ \\
\hline$m_{p}$ & mass of particle, $\mathrm{kg}$ \\
\hline$M$ & impeller torque, $\mathrm{N}-\mathrm{m}$ \\
\hline$M_{e}$ & number of independent voltage measurements \\
\hline$M I_{o}$ & overall mixing index (Equation (5.1-4)), dimensionless \\
\hline$M I_{r}$ & radial mixing index (Equation (5.1-3)), dimensionless \\
\hline$M I_{v}$ & mixing index (Equation (5.1-5)), dimensionless \\
\hline
\end{tabular}




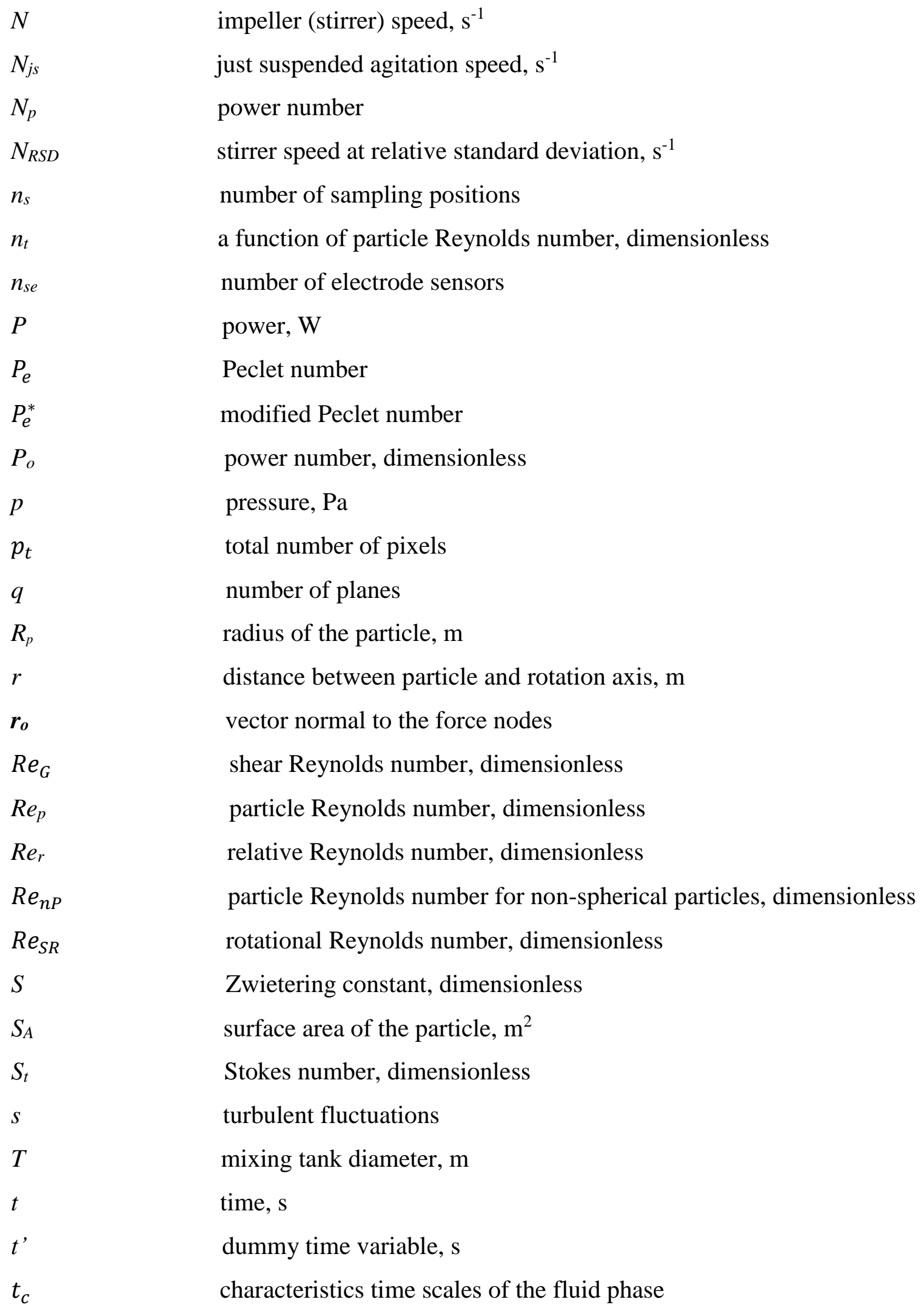




$\begin{array}{ll}t_{p r} & \text { particle response time, } \mathrm{s} \\ \vec{u}_{l}, \boldsymbol{u}_{c} & \text { fluid velocity, } \mathrm{m} / \mathrm{s} \\ \vec{u}_{p}, \boldsymbol{u}_{p} & \text { solid particle velocity, } \mathrm{m} / \mathrm{s} \\ \vec{u}_{q} & q^{\text {th }} \text { phase velocity, } \mathrm{m} / \mathrm{s} \\ u_{i,}^{\prime} u_{j}^{\prime} & \text { fluctuating components of velocity due to turbulence in the direction } i \text { and } j, \\ & \text { respectively, } \mathrm{m} / \mathrm{s} \\ U_{t o} & \text { terminal velocity at stagnant medium, } \mathrm{m} / \mathrm{s} \\ U_{t t} & \text { terminal velocity at turbulent medium, } \mathrm{m} / \mathrm{s} \\ u_{t p} & \text { particle terminal velocity, m/s } \\ V & \text { fluid stream velocity, } \mathrm{m} / \mathrm{s} \\ V_{c} & \text { velocity vector for continuous phase } \\ V_{p} & \text { velocity vector for particle phase } \\ v & \text { velocity, m/s } \\ V_{c} & \text { superficial velocity of the fluid, m/s } \\ V_{p} & \text { volume of particle, } \mathrm{m}^{3} \\ V_{t} & \text { free settling velocity of particle, } \mathrm{m} / \mathrm{s} \\ V_{t s} & \text { hindered settling velocity of particle, } \mathrm{m} / \mathrm{s} \\ v_{x}, v_{y}, v_{z} & \text { x, y, and z components of velocity } \\ \bar{v}_{x}, \bar{v}_{y}, \bar{v}_{z} & \text { x, y, and z components of mean velocity } \\ v_{x}^{\prime}, v_{y}^{\prime}, v_{z}^{\prime} & \text { x, y, and z fluctuating components of velocity } \\ w & \text { function of particle Reynolds number } \\ X & \text { solid particle weight fraction, dimensionless } \\ X_{p} & \text { position of a particle } \\ X_{v} & \text { average solid volume fraction, dimensionless } \\ X_{v c} & \text { height of planes, } \mathrm{m} \\ \bar{X}_{v} & \\ Y_{m} & \end{array}$




\section{Greek letters}

\begin{tabular}{|c|c|}
\hline$v, v_{c}$ & kinematic viscosity of the fluid, $\mathrm{m}^{2} / \mathrm{s}$ \\
\hline$\varepsilon$ & dissipation rate of turbulence, $\mathrm{m}^{2} / \mathrm{s}^{3}$ \\
\hline$\mu$ & viscosity, $\mathrm{Pa} . \mathrm{s}$ \\
\hline$\mu_{c}, \mu_{l}$ & continuous (liquid) phase viscosity, Pa. s \\
\hline$\mu_{q}$ & bulk viscosity, Pa. s \\
\hline$\mu_{q}^{(t)}, \mu_{q t}$ & eddy (turbulent) viscosity, Pa. s \\
\hline$\lambda$ & wave length, m \\
\hline$\rho$ & density, $\mathrm{kg} / \mathrm{m}^{3}$ \\
\hline$\rho_{c}$ & density of continuous phase, $\mathrm{kg} / \mathrm{m}^{3}$ \\
\hline$\rho_{l,} \rho_{L}$ & fluid density, $\mathrm{kg} / \mathrm{m}^{3}$ \\
\hline$\rho_{p,} \rho_{s}$ & density of solid particle, $\mathrm{kg} / \mathrm{m}^{3}$ \\
\hline$\rho_{q}$ & density of $q^{\text {th }}$ phase, $\mathrm{kg} / \mathrm{m}^{3}$ \\
\hline$\sigma$ & variance \\
\hline $\bar{\sigma}$ & mean conductivity value over whole selected volume, $\mathrm{mS} / \mathrm{cm}$ \\
\hline$\sigma_{e}$ & conductivity in each pixel, $\mathrm{mS} / \mathrm{cm}$ \\
\hline$\sigma_{l}$ & conductivity of the continuous (liquid) phase, $\mathrm{mS} / \mathrm{cm}$ \\
\hline$\sigma_{s}$ & conductivity of the particle phase, $\mathrm{mS} / \mathrm{cm}$ \\
\hline $\bar{\sigma}_{i}$ & average conductivity on the selected plane, $\mathrm{mS} / \mathrm{cm}$ \\
\hline$\sigma_{m c}$ & conductivity of the slurry, $\mathrm{mS} / \mathrm{cm}$ \\
\hline$\sigma_{k}, \sigma_{\varepsilon}$ & empirical constants \\
\hline$a_{q}$ & volume fraction of $q^{\text {th }}$ phase \\
\hline$a_{l}$ & liquid (continuous) phase volume fraction \\
\hline$a_{p}$ & particle (dispersed) phase volume fraction \\
\hline$\beta$ & empirical constant \\
\hline$\tau$ & stress tensor \\
\hline$\overline{\boldsymbol{\tau}}^{(v)}$ & viscous stress tensor \\
\hline$\overline{\boldsymbol{\tau}}^{(t)}$ & turbulent stress tensor \\
\hline$\tau_{p}$ & particle relaxation time, $\mathrm{s}$ \\
\hline
\end{tabular}




$\begin{array}{ll}\overline{\overline{\tau_{q}}} & q^{\text {th }} \text { phase stress-strain tensor } \\ \omega & \text { angular velocity, } \mathrm{rad} / \mathrm{s} \\ \Psi & \text { sphericity }\end{array}$

Abbreviations

\begin{tabular}{|c|c|}
\hline ASM & algebraic stress model \\
\hline CARPT & computer automated particles tracking \\
\hline CFD & computational fluid dynamics \\
\hline CT & computed tomography \\
\hline DAS & data acquisition system \\
\hline DEM & distinct element method \\
\hline DNS & direct numerical simulation \\
\hline $\mathrm{ECT}$ & electrical capacitance tomography \\
\hline EIT & electrical impedance tomography \\
\hline ERT & electrical resistance tomography \\
\hline ET & electrical tomography \\
\hline LDA & laser doppler anemometry \\
\hline LES & large eddy simulation \\
\hline LSIA & laser sheet and image analysis \\
\hline PBT & pitched blade turbine \\
\hline PIV & particle image velocimetry \\
\hline RANS & Reynolds Averaged Navier Stokes \\
\hline MRI & magnetic resonance imaging \\
\hline RMS & root mean square \\
\hline RSD & relative standard deviation \\
\hline RSM & Reynolds stress model \\
\hline $\mathrm{SG}$ & specific gravity \\
\hline SM & sliding mesh \\
\hline PEPT & positron emission particles tracking \\
\hline VFD & variable frequency drive \\
\hline
\end{tabular}




\section{Bibliography}

Aghajani, M., Muller-Steinhagen, H., Jamialahmadi, M., 2005. New design equations for liquid /solid fluidized bed heat exchangers. Int. J. Heat Mass Transfer 48, 317-329.

Alba, F., Crawley, G.M., Fatkin, J., Higgs, D.M.J., Kippax, P.G., 1999. Acoustic spectroscopy as a technique for the particle sizing of high concentration colloids, emulsions and suspensions. Colloids and Surfaces A: Physiochemical and Engineering Aspects 153, 495-502.

Alban, F.B., Sajjadi, S., Yianneskis, M., 2004. Dynamic tracking of fast liquid-liquid dispersion processes with a real time in situ optical technique. Chem. Eng. Res. Des. 82, 1054-1060.

Altobelli, S.A., Givler, R.C., Fukushima, E., 1991. Velocity and concentration measurements of suspensions by nuclear resonance imaging. J. Rheol. 35, 721.

Altway, A., Setyawan, H., Margono, M., Winardi, S., 2001. Effect of particle size on simulation of three-dimensional solid dispersion in stirred tank. Chem. Eng. Res. Des. 79(8), 1011-1016.

Ameur, H., Bouzit, M., Helmaoui, M., 2012. Hydrodynamic study involving a Maxblend impeller with yield stress fluids. J. Mech. Sci. Technol. 26 (5), 1523-1530.

Andreussi, P., Di Donfrancesco, A., Messia, M., 1988. An impedance method for the measurement of liquid hold-up in two phase flow. Int. J. Multiphase Flow 14, 777-787.

Andreussi, P., Paglianti, A., Silva, F., 1999. Dispersed bubble flow in horizontal pipes. Chem. Eng. Sci. 54, 1101-1107.

Angst, R., Kraume, M., 2006. Experimental investigations of stirred solid/liquid systems in three different scales: particle distribution and power consumption. Chem. Eng. Sci. 61, 2864-2870.

Armenante, P.M., Nagamine, E.U., 1998. Effect of low off-bottom impeller clearance on the minimum agitation speed for complete suspension of solids in stirred tanks. Chem. Eng. Sci. 53 (9), 1757-1775.

Asakura, K., Harada, S., Funayama, T., Nakajima, I., 1997. Simulation of descending particles in water by the distinct element method. Powder Technol. 94(3), 195-200. 
Ayranci, I., Tan, G., Dix-Cooper, M., Hossein-Pour, M., 2010. Mixing in Mineral Processing. CHE 420/620 mixing in process industries. North American Mixing Forum.

Baldi, G., Conti, R., Alaria, E., 1978. Complete suspension of particles in mechanically agitated vessels. Chem. Eng. Sci. 33 (1), 21-25.

Bamberger, J.A., Kytomaa, H.K., Greenwood, M.S., 1998. Slurry ultrasonic particle size and concentration characterization: W.W. Schultz, N.J. Lombardo (Eds.), Science and Technology for Disposal of Radioactive Tank Wastes, Plenum Press, New York.

Bamberger, J.A., Greenwood, M.S., 2004. Using ultrasonic attenuation to monitor slurry mixing in real time. Science Direct, 145-148.

Barigou, M., 2004. Particle tracking in opaque mixing systems: an overview of the capabilities of PET and PEPT. Chem. Eng. Res. Des. 82 (A9), 1258-1267.

Barressi, A., Baldi, G., 1987. Solid dispersion in an agitated vessel. Chem. Eng. Sci. 42, 29492956.

Barrue, H., Bertrand, J., Cristol, B., Xuereb, C., 2001. Eulerian simulation of dense solid-liquid suspension in multi-stage stirred vessel. J. Chem. Eng. Japan 34(5), 585-594.

Benbelkacem, H., Garcia-Bernet, D., Bollon, J., Loisel, D., Bayard, R., Steyer, J.P., Gourdon, R., Buffiere, P., Escudie, R., 2013. Liquid mixing and solid segregation in high-solid anaerobic digesters. Bioresour. Technol. 147, 387-394.

Bird, R.B., Stewart, W.E., Lightfoot, E.N., 2002. Transport Phenomena. $2^{\text {nd }}$ edition, John Wiley, New York.

Bittorf, K.J., Kresta, S.M., 2003. Prediction of cloud height for solid suspensions in stirred tanks. Chem. Eng. Res. Des. 81 (A5), 568-577.

Bjegovic, D., Krstic, V., Mikulic D., Ukrainczyk, V., 1995. C-D-c-t Diagrams for Practical Design of Concrete Durability Parameters. Cem. Concr. Res. 25 (1), 187-196.

Bohnet, M., Niesmak, G., 1980. Distribution of solids in stirred suspension. Ger. Chem. Eng. 3, $57-65$. 
Bolton, G.T., Primrose, K.M., 2005. An overview of electrical tomographic measurements in pharmaceutical and related applications areas. AAPS Pharm. Sci. Tech. 6 (2), 137-143.

Boyer, C., Duquenne, A.M., Wild, G., 2002. Measuring techniques in gas-liquid and gas-liquidsolid reactors. Chem. Eng. Sci. 57 (16), 3185-3215.

Brown, P.P., Lawler, D.F., 2003. Sphere drag and settling velocity revisited. J. Environ. Eng. 129(3), 222-231.

Brucato, A., Cipollina, A., Micale, G., Scargiali, F., Tamburini, A., 2010. Particle suspension in top covered unbaffled tanks. Chem. Eng. Sci. 65, 3001-3008.

Brunazzi, E., Paglianti, A., Pintus, S., 2001. A capacitance probe and a new model to identify and predict the capacity of columns equipped with structured packings. Ind. Eng. Chem. Res. 40, 12051212.

Brunazzi, E., Galletti, C., Paglianti, A., Pintus, S., 2004. An impedance probe for the measurements of flow characteristics and mixing properties in stirred slurry reactors. Chem. Eng. Res. Des. 82, 1250-1257.

Bubicco, R, Di Cave, S., Mazzarotta, B., 1997. Influence of solid concentration and type of impeller on the agitation of large PVC particles in water. Recents Prog. Genie Procedes, 11 (52), 81.

Bujalski, W., Takenaka, K., Paolini, S., Jahoda, M., Paglianti, A., Takahashi, K. 1999. Suspension and liquid homogenization in high solids concentration stirred chemical reactors. Chem. Eng. Res. Des. 77 (A3), 241-247.

Buurman, C., Resoort, G., Plaschkes, A., 1985. Scaling up rules for solids suspension in stirred vessels. Proceedings of the 5th European Conference on Mixing.

Carletti, C., Montante, G., Westerlund, T., Paglianti, A., 2014. Analysis of solid concentration distribution in dense solid-liquid stirred tanks by electrical resistance tomography. Chem. Eng. Sci. 119, 53-64.

Caulet, P.J.C., Van der Lans, R.G.J.M., Luyben, K.Ch.A.M, 1996. Hydrodynamical interactions between particles and liquid flows in biochemical applications. Chem. Eng. J. 62, 193-206. 
Chang, J.S., Ichikawa, Y., Irons, G.A., Morala, E.C., Wan, P.T., 1984. Void fraction measurement by an ultrasonic transmission technique in bubbly gas-liquid two-phase flow: Delhaye, J.M., Cognet, G. (Eds.), Measuring Techniques in Gas-Liquid Two-phase Flows. Springer, New York. Chaouki, J., Larachi, F., Dudukovic, M.P. (Eds.), 1997. Non-intrusive monitoring of multiphase flows. Elsevier Science B.V., Amsterdam.

Chapman, C.M., 1981. Studies of gas-liquid-particle mixing in stirred vessels. PhD Thesis, University College London.

Chapman, C.M., Nienow, A.W., Cooke, M., Middleton, J.C., 1983. Particle-gas-liquid mixing in stirred vessels - Part 1: particle liquid mixing. Chem. Eng. Res. Des. 61, 71-81.

Chen, X.Q., Pereira, J.C.F., 2000. Computational modeling of a dilute turbulent liquid-solid flow using a eulerian-lagrangian approach. Int. J. Numer. Methods Heat Fluid Flow 10(4), 409-431.

Chen, J., Xiao, W., 2013. Solids suspension study in a side-entering stirred tank through CFD modeling. Int. J. Chem. Reactor Eng. 11(1), 1-16.

Chien, S.F., 1994. Settling velocities of irregular shaped particles. SPE Srilling and Compilation, 9, 281.

Clift, R., Grace, J.R., Weber, M.E., 1978. Bubbles, drops, and particles. Academic Press, New York.

Cokljat, D., Slack, M., Vasquez, S.A., Bakker, A., Montante, G. Reynolds-stress model for Eulerian multiphase. Progress Comput. Fluid Dyn. 6, 168.

Crowe, C.T., Elger, D.F., Williams, B.C., Roberson, J.A., 1998. Engineering Fluid Mechanics, Ninth Edition, Wiley.

Cullen, P.J., 2009. Food Mixing: Principles and Applications. First edition. Wiley-Blackwell. Dallavalle, J.M., 1948. Micrometrics. Pitman, London.

Decker, S., Sommerfeld, M., 1996. Calculation of particle suspension in agitated vessels with the euler-lagrange approach. Proceedings of the 1996 Fluid Mixing 5 Conference 140, 71-82. 
Derksen, J.J., 2003. Numerical simulation of solids suspension in a stirred tank. AIChE J. 49, 2700-2714.

Dickin, F., Wang, M., 1996. Electrical resistance tomography for process applications. Meas. Sci. Tech. 7(3), 247-260.

Ditl, P., Rieger, F., 1985. Suspension of solids particle-relative velocity of particles in turbulent mixing. Proceedings of the 5th European Conference on Mixing, Wurzburg, West Germany.

Doh, D.H., Yum, J.H., Cho, G.R., Kim, M.H., Ryu, G.W., Takei, M., 2013. Development of a concentration measurement technique for steady state solid-liquid mixing using a neural network. J. Therm. Sci. 22 (5), 478-483.

Drewer, G.R., Ahmed, N., Jameson, G.J., 1994. Suspension of high concentration solids in mechanically stirred vessels. AIChE Symp. Ser. 136, 41.

Dudukovic, M.P., 2002. Opaque multiphase flows: experiments and modelling. Exp. Therm. Fluid Sci. 26, 747-761.

Duim, W.C., Clarke, S.M., 2006. Adsorption and mixing behaviour of ethers and alkanes at the solid/liquid Interface. J. Phys. Chem. B. 110, 23853-23859.

Dyakowski, T., Jeanmeure, L.F.C., Jaworski, A.J., 2000. Applications of electrical tomography for gas-solids and liquid-solids flows - a review. Powder Technol. 112, 174-192.

Einenkel, W.D., 1980. Influence of physical properties and equipment design on the homogeneity of suspensions in agitated vessels. Ger. Chem. Eng. 3, 118-124.

Fajner, D., Magelli, F., Nocentini, M., Pasquali, G., 1985. Solids concentration profiles in a mechanically stirred and staged column slurry reactor. Chem. Eng. Res. Des. 63, 235-240.

Fan, L., Mao, Z., Wang, Y., 2005. Numerical simulation of turbulent solid-liquid two-phase flow and orientation of slender particles in a stirred tank. Chem. Eng. Sci. 60, 7045-7056.

Fangary, Y.S., Barigou, M., Seville, J.P.K., Parker, D.J., 2000. Fluid trajectories in a stirred vessel of non-Newtonian liquid using positron emission particle tracking. Chem. Eng. Sci. 55, 59695979. 
Fathi Roudsari, S., Dhib, R., Ein-Mozaffari, F., 2016. Using a novel CFD model to assess the effect of mixing parameters on emulsion polymerization. Macromol. React. Eng. 10 (2), 108-122. Fergizer, J.H., Peric, M., 1996. Computational methods for fluid dynamics. Springer, USA.

Ferreira, P.J., Rasteiro, M.G., Figueiredo, M.M., 1994. A new approach to measuring solids concentration in mixing tanks. Adv. Powder Technol. 5 (1), 15-24.

Ferry, J., Balachandar, S., 2001. A fast Eulerian method for disperse two-phase flow. Int. J. multiphase flow, 27, 1199-1226.

Flemmer, R.L.C., Banks, C.L., 1986. On the drag coefficient of a sphere. Powder Technol., 48, 217-221.

Fluent web assistant, 2006. Fluent user's guide documentation. Fluent Inc.

Foucault, S., Ascanio, G., Tanguy, P.A., 2004. Coaxial mixer hydrodynamics with Newtonian and non-Newtonian fluids. Chem. Eng. Technol. 27 (3).

Fournier, T., Jeandey, C., 1993. Optimization of an experimental setup for void fraction determination by the X-ray attenuation technique, Delhayr, J.M., Cognet, G. (Eds.), Measuring techniques in gas-liquid two-phase flows, 199-228, New York.

Fradette, L., Tanguy, P.A., Bertrand, F., Thibault, F., Ritz, J., Giraud, E., 2007. CFD phenomenological model of solid-liquid mixing in stirred vessels. Comput. Chem. Eng. 31(4), 334-345.

Ganser, G.H., 1993. A rational approach to drag prediction of spherical and non-spherical particles. Powder Technol. 77, 143-152.

Garside, J., Al-Dibouni, M.R., 1977. Velocity-voidage relationships for fluidization and sedimentation in solid-liquid systems. Ind. Eng. Chem. Proc. Des. Dev. 16(2), 206-214.

Gerstenberg, H., Sckuhr, P., Steiner, R., 1983. Stirred tank reactors for polymerization. Ger. Chem. Eng. 6, 129-141.

Gidaspow, D., Bezburuah, R., Ding, J., 1992. Hydrodynamics of circulating fluidized beds, kinetic theory approach. In Fluidization VII, Proceedings of the $7^{\text {th }}$ Engineering Foundation Conference on Fluidization, 75-82. 
Gidaspow, D., 1994. Multiple flow and fluidization: Continuum and kinetic theory descriptions. Academic Press, Boston.

Giguere, R., Fradette, L., Tanguy, P.A., Mignon, D. (2008). Characterization of slurry flow regime transitions by ERT. Chem. Eng. Res. Des. 86(9), 989-996.

Godfrey, J.C., Zhu, Z.M., 1994. Measurement of particle-liquid profiles in agitated tanks. AIChE Symp. Ser. 299, 90:181.

Gohel, S., Joshi, S., Azhar, M., Horner, M., Padron, G., 2012. CFD modeling of solid suspension in a stirred tank: Effect of drag models and turbulent dispersion on cloud height. I. J. Chem. Eng. ID 956975.

Guha, D., Ramachandran, P.A., Dudukovic, M.P., 2007. Flow field of suspended solids in a stirred reactor by Lagrangian tracking. Chem. Eng. Sci. 62, 6143-6154.

Guida, A., Fan, X., Parker, D.J., Nienow, A.W., Barigou, M., 2009. Positron emission particle tracking in a mechanically agitated soli-liquid suspension of coarse particles. Chem. Eng. Res. Des. 87 (4), 421-429.

Guida, A., Nienow, A.W., Barigou, M., 2010. PEPT measurements of solid-liquid flow field and spatial phase distribution in concentrated mono disperse stirred suspensions. Chem. Eng. Sci. 65 (6), 1905-1914.

Guillard, F., Tragardh, C., Fuchs, L. 2000. A study of turbulent mixing in a turbine-agitated tank using a fluorescence technique. Exp. in Fluids 28, 225-235.

Guo, H.M., Wang, L.G., Wang, Q., Yang, X.J., 2014. Effects of Solid-Liquid Mixing on Microstructure of Semi-Solid A356 Aluminium Alloy. The Minerals, Metals \& Materials Society and ASM International 45B, 1490.

Haider, A., Levenspiel, O., 1989. Drag coefficient and terminal velocity of spherical and nonspherical particles. Powder Technol. 58, 63-70.

Hao, Z., 2005. CFD simulation of segregation in particulate multiphase flows with binary solids. Proceedings of the American Society of Mechanical Engineers, Fluids Engineering Division Summer Conference, 669-676. 
Harrison, S.T.L., Stevenson, R., Cilliers, J.J., 2012. Assessing solids concentration homogeneity in Rushton-agitated slurry reactors using electrical resistance tomography (ERT). Chem. Eng. Sci. 71, 392-399.

Hartman, M., Trnka, O., Svoboda, K., 1994. Free settling of non-spherical particles. Ind. Eng. Chem. Res. 33, 1979-1983.

Hemrajani, R.R., et al., 1998. Suspending floating solids in stirred tanks: mixer design, scale-up and optimization. Proc. $6^{\text {th }}$ European conference on mixing, May 24-26, 259-265.

Hewitt, G.F., 1978. Measurement of two phase flow parameters. Academic Press, New York.

Hicks, M.T., Myers, K.J., Bakker, A., 1996. Cloud height in solid suspension agitation. Chem. Eng. Commun. 160, 137-155.

Hormann, T., Suzzi, D., Khinast, J.G., 2011. Mixing and dissolution processes of pharmaceutical bulk materials in stirred tanks: Experimental and numerical investigations. Ind. Eng. Chem. Res. 50, 12011-12025.

Hosseini, S., Patel, D., Ein-Mozaffari, F., Mehrvar, M., 2010a. Study of solid-liquid mixing in agitated tanks through computational fluid dynamics modeling. Ind. Eng. Chem. Res. 49(9), 44264435 .

Hosseini, S., Patel, D., Ein-Mozaffari, F., Mehrvar, M., 2010b. Study of solid-liquid mixing in agitated tanks through electrical resistance tomography. Chem. Eng. Sci. 65, 1374-1384.

Houcine, I., Vivier, H., Plasari, E., David, R., Villermaux, J., 1996. Planar laser induced fluorescence technique for measurements of concentration fields in continuous reactors. Exp. in Fluids 22, 96-102.

Hua, P., Woo, E.J., Webster, J.G., Tompkins, W.J., Inc, S.G., Estates, H., 1993. Using compound electrodes in electrical impedance tomography. Biomedical Eng., IEEE Trans., 40(1), 29-34.

Jafari, R., Tanguy, P.A., Chaouki, J., 2012. Experimental investigation on solid dispersion, power consumption and scale-up in moderate to dense solid-liquid suspensions. Chem. Eng. Res. Des. 90, 201-212. 
Joosten, G.E.H., Schilder, J.G.M., Broere, A.M., 1997. The suspension of floating solids in stirred vessels. Trans. Inst. Chem. Eng., 55, 220.

Joshi, J.B., Nere, N.K., Rane, C.V., Murthy, B.N., Mathpati, C.S., Patwardhan, A.W., Ranade, V.V., 2011. CFD simulation of stirred tanks: Comparison of turbulence models. Part I: Radial flow impellers. Can. J. Chem. Eng. 89, 23-82.

Kapishnikov, S., Kantsler, V., Steinberg, V., 2006. Continuous particle size separation and size sortening using ultrasound in a microchannel. Journal of Statical Mechanics: Theory and Experiment, 1012, 1-15.

Kasat, G.R., Khopkar, A.R., Ranade, V.V., Pandit, A.B., 2008. CFD simulation of liquid phase mixing in solid-liquid stirred reactor. Chem. Eng. Sci., 63(15), 3877-3885.

Kaufmann, A., Simonin, O., Poinsot, T., Helie, J., 2002. Dynamics and dispersion in eulerianeulerian DNS of two-phase flows. Center for Turbulence Research, Proceedings of the summer program, 381-392.

Kazemzadeh, A., Ein-Mozaffari, F., Lohi, A., Pakzad, L., 2016. Investigation of hydrodynamic performances of co-axial mixers in agitation of yield-pseudoplastic fluids: Single and double central impellers in combination with the anchor. Chem. Eng. J. 294, 417-430.

Kee, N.C.S., Tan, R.B.H., 2002. CFD simulation of solids in mixing vessels. Can. J. Chem. Eng. 80(4), 721-726.

Khan, A.R., Richardson, J.F., 1987. The resistance to motion of a solid sphere in a fluid. Chem. Eng. Comm., 62, 135-150.

Khare, A.S., Dharwadkar, S.V., Joshi, J.B., Sharma, M.M., 1990. Liquid and solid phase mixing in a sectionalized bubble column slurry reactor. Ind. Eng. Chem. Res. 29, 1503-1509.

Khopkar, A.R., Kasat, G.R., Pandit, A.B., Ranade, V.V., 2006. Computational fluid dynamics simulation of the solid suspension in a stirred slurry reactor. Ind. Eng. Chem. Res. 45(12), 44164428.

Kim, I., Elghobashi, S., Sirignano, W.A., 1998. On the equation for spherical-particle motion: Effect of Reynolds and acceleration numbers. J. Fluid Mech. 367, 221-253. 
Kleinstreuer, C., 2003. Two-phase flow: Theory and applications. Taylor and Francis Books Inc. Kodialbail, V.S., Srinikethan, G., 2011. Mixing and solid-liquid mass transfer characteristics in a three phase pulsed plate column with packed bed of solids in interpolate spaces - a novel aerobic immobilized cell bioreactor. J Chem. Technol. Biotechnol. 86, 1310-1320.

Kolar, V., 1961. Suspending solid particles in liquids by means of mechanical agitation. Coll. Czech. Chem. Commun. 26, 613-627.

Kondo, S., Yamada, N., Takahashi, K., 2008. Distribution of solid particles lighter than liquid in an agitated vessel stirred by dual impellers. Japan J. Chem. Eng. 41 (3), 155-160.

Kraume, M., 1992. Mixing times in stirred suspensions. Chem. Eng. Tech., 15(5), 313-318.

Kresta, S.M., Wood, P.E., 1993. The mean flow field produced by a $45^{0}$ pitched blade turbine: changes in the circulation pattern due to off bottom clearance. Can. J. Chem. Eng. 71 (1), 42-53.

Kumar, S.B., Moslemian, D., Dudukovic, M.P., 1995. A $\gamma$-ray tomographic scanner for imaging of void distribution in two-phase flow systems. Flow Meas. Instrum. 6 (3), 61-73.

Kuzmanic, N., Kessler, E.M., 1997. Continuous sampling of floating solids suspension from a mixing tank. Ind. Eng. Chem. Res. 36 (11), 5015-5022.

Larachi, F., Chaouki, J., Kennedy, G., Dudukovic, M.P., 1997. Radioactive particle tracking in multiphase reactors: principles and applications. Non-Intrusive Monit. Multiphase Flows, 335406.

Launder, B.E., Spalding, D.B., 1972. Lectures in mathematical models of turbulence. Academic Press, London.

Ljungqvist, M., Rasmuson, A., 2001. Numerical simulation of the two-phase flow in an axially stirred vessel. Chem. Eng. Res. Des., 79(A5), 533-546.

Liang, W., Jin, Y., Yu, Z., Wang, Z., Zhu, J., Chen, J., 1996. Flow characteristics and mixing properties in a high velocity liquid-solid loop reactor. Chem. Eng. J. 63, 181-188.

Lu, W.M., Hsu, R.C., Chien, W.C., Lin, L.C., 1993. Measurement of local bubble diameters and analysis of gas dispersion in an aerated vessel with disk-turbine impeller. J. Chem. Eng. Japan 26, 551-557. 
Lu, W.M., Wu, H.Z., Ju, M.Y., 1997. Effects of baffle design on the liquid mixing in an aerated stirred tank with standard Rushton turbine impellers. Chem. Eng. Sci., 52 (21-22), 3843-3851.

Machado, M.B., Bittorf, K.J., Roussinova, V.T., Kresta, S.M., 2013. Transition from turbulent to transitional flow in the top half of a stirred tank. Chem. Eng. Sci. 98, 218-230.

MacTaggart, R.S., Nasr-El-Din, H.A., Masliyah, J.H., 1993. Sample withdrawal from a slurry mixing tank. Chem. Eng. Sci. 48, 921-931.

Madupu, A., Mazumdar, A., Jinsong, Z., Roelant, D., Srivastava, R., 2005. Electrical resistance tomography for real-time mapping of the solid-liquid interface in tanks containing optically opaque fluids. Proceedings of the SPIE- Int. Soc. Optical Eng., 5674(1), 36-46.

Magelli, F., Fajner, D., Nocentini, M., Pasquali, G., 1987. Novel data about solids concentration distribution in vessels stirred with multiple impellers. CHISA 87, Paper E5.5, Prague.

Magelli, F., Fajner, D., Nocentini, M., Pasquali, G., Marisko, V., Ditl, P., 1991. Solids concentration distribution in slurry reactors stirred with multiple axial impellers. Chem. Eng. Proc. $29,27-32$.

Majors, P.D., Givler, R.C., Fukushima, E., 1989. Velocity and concentration measurements in multiphase flows by NMR. J. Magn. Reson. 85 (2), 235-243.

Mak, A.T.C., 1992. Solid-liquid mixing in mechanically agitated vessels. Ph.D. Dissertation, University of London.

Mann, R, Williams, R.A., Dyakowski, T., Dickin, F.J., Edwards, R.B., 1997. Development of mixing models using electrical resistance tomography. Chem. Eng. Sci. 52 (13), 2073-2085.

Maude, A.D., Whitmore, R.L., 1958. A generalised theory of sedimentation. Br. J. Appl. Phys. 9, 477-482.

McKee, S.L., Williams, R.A., Boxman, A., 1995. Development of solid-liquid mixing models using tomographic techniques. Chem. Eng. J. 56, 101-107.

Mei, C.C., 1992. Method of homogenization applied to dispersion in porous media. Transport in porous media $9(3), 261-274$. 
Mersmann, A., Werner, F., Maurer, S., Bartosch, K., 1998. Theoretical prediction of the minimum stirrer speed in mechanically agitated suspensions. Chem. Eng. Proc. 37 (6), 503-510.

Micale, G., Carrara, V., Grisafi, F., Brucato, A., 2000. Solids suspension in three-phase stirred tanks. Chem. Eng. Res. Des. 78 (3), 319-326.

Micale, G., Grisafi, F., Rizzuti L., Brucato, A., 2004. CFD Simulation of Particle Suspension Height in Stirred Vessels. Chem. Eng. Res. Des. 82 (A9), 1204-1213.

Micheletti, M., Nikiforaki, L., Lee, K.C., Yianneskis, M., 2003. Particle concentration and mixing characteristics of moderate-to-dense solid-liquid suspensions. Ind. Eng. Chem. Res. 42, 62366249.

Micheletti, M., Yianneskis, M., 2004. Study of fluid velocity characteristics in stirred solid-liquid suspensions with a refractive index matching technique. Part E: J. Proc. Mech. Eng. IMechE 218 (4), 191-204.

Milliken, W.J., Gottlieb, M., Graham, A.L., Mondy, L.A., Powell, R.L., 1989. The viscosityvolume fraction relation for suspensions of randomly oriented rods. J. Fluid Mech. 202, 217.

Molerus, O., Latzel, W., 1987a. Suspension of solid particles in agitated vessels - I. Archimedes numbers $\leq 40$. Chem. Eng. Sci. 42 (6), 1423-1430.

Molerus, O., Latzel, W., 1987b. Suspension of solid particles in agitated vessels - II. Archimedes numbers $>40$, reliable prediction of minimum stirrer angular velocities. Chem. Eng. Sci. 42 (6), 1431-1437.

Mondy, L.A., Graham, A.L., Majumdar, A., Bryant, 1986. Techniques of measuring particle motions in concentrated suspensions. Int. J. Multiphase Flow 12, 497.

Montante, G., Lee, K.C., Brucato, A., Yianneskis, M., 1999. Double- to single -loop flow pattern transition in stirred vessels. Can. J. Chem. Eng. 77, 649.

Montante, G., Micale, G., Magelli, F., Brucato, A., 2001. Experiments and CFD predictions of solid particle distribution in a vessel agitated with four pitched blade turbines. Trans IChemE 79 (A), 1005-1010. 
Morsi, S.A., Alexander, A.J., 1972. Theoretical low-speed particles collision with symmetrical and cambered aerofoils. ASME, 72 (35), 12.

Mott, R. L., 2005. Applied Fluid Mechanics Sixth Edition. Prentice-Hall, New York.

Murthy, B.N., Ghadge, R.S., Joshi, J.B., 2007. CFD simulations of gas-liquid-solid stirred reactor: Prediction of critical impeller speed for solid suspension. Chem. Eng. Sci. 62, 7184-7195.

Musil, L., Vlk, J., 1978. Suspending solid particles in an agitated conical-bottom tank. Chem. Eng. Sci. 33 (8), 1123-113.

Musil, L., Vlk, J., Jiroudkova, H., 1984. Suspending solid particles in an agitated tank with axial type impellers. Chem. Eng. Sci. 39 (4), 621-628.

Myers, K.J., Herr, J.P., Janz, E.E., 2011. Solids suspension with angle-mounted agitators in unbaffled vessels. Can. J. Chem. Eng. 89, 940-947.

Narayanan, S., Bhatia, V.K., Guha, D.K., Rao, M.N., 1969. Suspension of solids by mechanical agitation. Chem. Eng. Sci. 24 (2), 223-230.

Nasr-El-Din, H.A., MacTaggart, R.S., Masliyah, J.H., 1996. Local solids concentration measurement in a slurry mixing tank. Chem. Eng. Sci. 51 (8), 1209.

Nienow, A.W., 1985. The dispersion of solids in liquids, in mixing of liquids by mechanical agitation. J.J. Ulbrecht and G.K. Patterson, eds., Gordon \& Breach, New York, 273-307.

Ochieng, A., Lewis, A.E., 2006. CFD simulation of solids off-bottom suspension and cloud height. Hydrometallurgy 82, 1-12.

Ochieng, A., Onyango, M.S., Kiriamiti, H.K., 2009. Experimental measurement and CFD simulation of mixing in a stirred tank: A review, S.A.J. Sci. Tech., 105, 421-426.

Oldshue, J.Y., 1983. Fluid Mixing Technology. McGraw Hill, New York.

Oshinowo, L.M., Bakker, A., 2002. CFD modeling of solids suspensions in stirred tanks. Presented at the TMS annual meeting, Seattle, WA, p 215.

Paglianti, A., Pintus, S., 2001. The impedance probe for the measurements of liquid hold-up and mixing time in two/three-phase stirred tank reactors. Exp. in fluids, 31, 417-427. 
Pakzad, L., Ein-Mozaffari, F., Chan, P., 2008. Measuring mixing time in the agitation of nonNewtonian fluids through electrical resistance tomography. Chem. Eng. Technol. 31, 1838-1845.

Panneerselvam, R., Savithri, S., Surender, G.D., 2008. CFD modeling of gas-liquid-solid mechanically agitated contactor. Chem. Eng. Res. Des. 86, 1331-1344.

Parker, D.J., Broadbent, C.J., Fowles, P., Hawkesworth, M.R., McNeil, P., 1993. Positron emission particle tracking - A technique for studying flow within engineering equipment. Nuclear Instruments and Methods in Physics Research 326 (A), 592-607.

Paul, E.L., Atiemo-Obeng, V.A., Kresta, S.M., 2004. Handbook of Industrial Mixing: Science and Practice. Wiley-Interscience, New York.

Pavulshenko, I.S., Kostin, N.M., Matveev, M.S., 1957. Stirrer speeds in stirring of suspensions. USSR. Appl. J. Chem. 30, 1235-1243.

Pekker, S.M., Helvaci, S.S., 2008. Solid-liquid two phase flow. Elsevier Ltd., UK.

Penaz, F., Rod, V., Rehakova, M., 1978. Concentration distribution of suspension in a mixed vessel. Coll. Czech. Chem. Commun. 43, 848-861.

Perkins, E.D., Williams, J.R., 2001. Generalized spatial binning of bodies of different sizes. In Discrete Element Methods: Numerical Modeling of Discontinua. ASCE, USA.

Perry, R.H., Green, D., 1984. Chemical Engineers Handbook. McGraw Hill, New York.

Pinelli, D., Nocentini, M., Magelli, F., 2001. Solids distribution in stirred slurry reactors: influence of some mixer configurations and limits to applicability of a simple model for predictions. Chem. Eng. Comm., 188, 91-107.

Powell, R.L., 2008. Experimental techniques for multiphase flows, Physics of fluids 20, 040605.

Prat, O.P., Ducoste, J.J., 2007. Simulation of flocculation in stirred vessels - lagrangian versus eulerian. Chem. Eng. Res. Des. 85(A2), 207-219.

Raghava Rao, K.S.M.S., Rewatkar, V.B., Joshi, J.B., 1988. Critical impeller speed for solid suspension in mechanically agitated contactors. AIChE J., 34, 1332. 
Rammohan, A.R., Kemoun, A., Al-Dahhan, M.H., Dudukovic, M.P., 2001. Characterization of single phase flows in stirred tanks via computer automated radioactive particle tracking (CARPT). Transactions of the Institution of Chemical Engineers 79 (A), 831-844.

Rammohan, A.R., Dudukovic, M.P., Ranade, V.V., 2003. Eulerian flow field estimation from particle trajectories: numerical experiments for stirred tank type flows. Ind. Eng. Chem. Res. 42, 2589-2601.

Ranade, V.V., 2002. Computational flow modeling for chemical reactor engineering. Academic Press, New York.

Ranade, V.V., Tayalia, Y., Krishnan, H., 2002. CFD predictions of flow near impeller blades in baffled stirred vessels: Assessment of computational snapshot approach. Chem. Eng. Comm., 189(7), 895-922.

Rani, S.L., Balachandar, S., 2004. Preferential concentration of particles in isotropic turbulence: a comparison of the Lagrangian and the equilibrium Eulerian approaches. Powder Technol. 141, 109-118.

Ren, C., Jiang, X., Wang, J., Yang, Y., Zhang, X., 2008. Determination of critical speed for complete solid suspension using acoustic emission method based on multi scale analysis in stirred tank. Ind. Eng. Chem. Res. 47, 5323-5327.

Rewatkar, V.B., Raghava Rao, K.S.M.S., Joshi, J.B., 1991. Critical impeller speed for solid suspension in mechanically agitated three-phase reactors. 1. Experimental Part, Ind. Eng. Chem. Res. 30 (8), 1770-1784.

Ricard, F., Brechtelsbauer, C., Xu, X.Y., Lawrence, C.J., 2005. Monitoring of multiphase pharmaceutical processes using electrical resistance tomography. Chem. Eng. Res. Des., 83(7), 794-805.

Ro, K.S., Neethling, J.B., 1990. Terminal settling characteristics of bio-particles. Research Journal of the Water Pollution Control Federation, 62, 901-906.

Rodgers, T.L., Stephenson, D.R., Cooke, M., York, T.A., Mann, R., 2009. Tomographic imaging during semi-batch reactive precipitation of barium sulphate in a stirred vessel. Chem. Eng. Res. Des. 87, 615-626. 
Rubinow, S.I., Keller, J.B., 1961. Shift of the shadow boundary and scattering cross section of an opaque object. J. Appl. Phys. 32(5), 814-820.

Rushton, J.H., 1965. The continuous removal of mixed phases from a mixing tank. AIChE I. Chem. Eng. Symp. Ser. 10, 3-10.

Saffman, P.G., 1965. The lift on a small sphere in a slow shear flow. J. Fluid Mech. 22, 385-400.

Sardeshpande, M.V., Sagi, A.R., Juvekar, V.A., Ranade, V.V., 2009. Solid suspension and liquid phase mixing in solid-liquid stirred tanks. Ind. Eng. Chem. Res. 48, 9713-9722.

Sardeshpande, M.V., Ranade, V.V., 2012. Computational fluid dynamics modeling of solid suspension in stirred tanks. Current Sci. 102(11), 1539-1551.

Schmitz, D., Mewes, D., 2000. Tomographic imaging of transient multiphase flow in bubble columns. Chem. Eng. J. 77, 99-104.

Scully, J., Frawley, P., 2011. Computational fluid dynamics analysis of the suspension of nonspherical particles in a stirred tank. Ind. Eng. Chem. Res. 50, 2331-2342.

Sessiecq, P., Mier, P., Gruy, F., Cournil, M., 1999. Solid particles concentration profiles in an agitated vessel. Institution of Chemical Engineers, Trans IChemE 77 (A), 741-746.

Sha, Z.L., Palossari, S., Oinas, P., Ogawa, K., 2001. CFD simulation of solid suspension in a stirred tank. J. Chem. Eng. Jpn. 34(5), 621-626.

Shamlou, P.A., Zolfagharian, A., 1987. Incipient solid motion in liquids in mechanically agitated vessels. Proceedings of the 3rd Fluid Mixing Conference 195-208.

Shamlou, P.A., Koutsakos, E., 1989. Solids suspension and distribution in liquids under turbulent agitation. Chem. Eng. Sci. 44 (3), 529-542.

Shan, X., Yu, G.Z., Yang, C., Mao, Z.S., Zhang, W., 2008. Numerical simulation of liquid-solid flow in an unbaffled stirred tank with a pitched-blade turbine down flow. Ind. Eng. Chem. Res. 47, 2926-2940.

Sharifi, M., Young, B., 2013. Electrical Resistance Tomography (ERT) applications to chemical engineering. Chem. Eng. Res. Des. 91 (9), 1625-1645. 
Sharma, R.N., Das, H.C.L., 1980. Effect of withdrawal flow velocity on the composition of a two phase system in a mixing tank. Coll. Czech. Chem. Commun. 45, 3293-3301.

Shirhatti, V., Wang, M., Williams, R., Ortega, J.R., 2006. Determination of minimum agitation speed for complete solid suspension using four electrode conductivity method. 5th International symposium on Measurement Techniques for multi-phase flows, Macau, China.

Shrotri, S., Harris, C.C., Huang, L., Somasundaran, P., 1998. A graphical technique for calculating adsorption/desorption isotherms for different solid/liquid ratios. Colloids and Surfaces A: Physiochemical and Engineering Aspects 141, 189-192.

Siddiqui, H., 1993. Mixing technology for buoyant solids in a non-standard vessel. AIChE J. 39(3), 505.

Sinton, S.W., Chow, A.W., 1991. NMR flow imaging of fluids and solid suspensions in Poiseuille flow. J. Rheol. 35, 735.

Sommerfeld, M., Decker, S., 2004. State of the art and future trends in CFD simulation of stirred vessel hydrodynamics. Chem. Eng. Technol. 27(3), 215-224.

Spidla, M., Sinevic, V., Jahoda, M., Machon, V., 2005. Solid particles distribution of moderately concentrated suspensions in a pilot plant stirred vessel. Chem. Eng. J. 113, 73-82.

Spidla, M., Mostek, M., Sinevic, V., Jahoda, M., Machon, V., 2005. Experimental assessment and CFD simulations of local solid concentration profiles in a pilot-scale stirred tank. Chem. Pap. 59, 386-393.

Srinivasa, T., Jayanti, S., 2007. An Eulerian/Lagrangian study of solid suspension in stirred tanks. AICHE J. 53, 2461-2469.

Stanley, S.J., Mann, R., Primrose, K., 2002. Tomographic imaging of fluid mixing in three dimensions for single-feed semi-batch operation of a stirred vessel. Trans IChemE 80 (A), 903909.

Stanley, S.J., 2006. Tomographic imaging during reactive precipitation in a stirred vessel: mixing with chemical reaction. Chem. Eng. Sci. 61(24), 7850-7863. 
Stapf, S., Han, S., 2006. Nuclear Magnetic Resonance Imaging in Chemical Engineering. Wiley$\mathrm{VCH}$, Weinheim, Germany.

Stephenson, D.R., Mann, R., York, T.A., 2008. The sensitivity of reconstructed images and process engineering metrics to key choices in practical electrical impedance tomography. Meas. Sci. Technol. 19, 094013 (15).

Subbarao, D., Taneja, V.K., 1979. Three phase suspensions in agitated vessels. Proceedings of the 3rd European Conference on Mixing, York, U.K.

Susanto, J., 1989. Multiple phase soli-liquid and solid-liquid-gas mixing in stirred tanks. MSc Thesis, New Jersey Institute of Technology.

Swamee, P.K., Ojha, C.P., 1991. Drag coefficient and fall velocity of non-spherical particles. J. Hydraulic Eng. 117, 409-419.

Syamlal, M., O'Brien, T.J., 1989. Computer simulation of bubbles in a fluidized bed. AIChE Symp. Ser. 85(270), 22-31.

Tahvildarian, P., Ng, H., D’Amato, M., Drappel, S., Ein-Mozaffari, F., Upreti, S.R., 2011. Using electrical resistance tomography images to characterize the mixing of micron-sized polymeric particles in a slurry reactor. Chem. Eng. J. 172, 517-525.

Takenaka, K., Takahashi, K., 1996. Local gas hold-up and recirculation rate in an aerated vessel equipped with a Rushton impeller. J. Chem. Eng. Japan 29, 799-804.

Tamburini, A., Gentile, L., Cipollina, A., Micale, G., Brucato, A., 2009. Experimental investigation of dilute solid-liquid suspension in an unbaffled stirred vessel by a novel pulsed laser based image analysis technique. Chem. Eng. Tran. 17, 531-536.

Tamburini, A., Cipollina, A., Micale, G., Brucato, A., Ciofalo, M., 2011. CFD simulations of dense solid-liquid suspensions in baffled stirred tanks: predictions of suspension curves. Chem. Eng. J. $178,324-341$.

Tamburini, A., Cipollina, A., Micale, G., Brucato, A., Ciofalo, M., 2013. CFD simulations of dense solid-liquid suspensions in baffled stirred tanks: Prediction of solid particle distribution. Chem. Eng. J. 223, 875-890. 
Tamburini, A., Brucato, A., Busciglio, A., Cipollina, A., Grisafi, F., Micale, G., Scargiali, F, Vella, G., 2014. Solid-liquid suspension in top covered unbaffled vessels: influence of particle size, liquid viscosity, impeller size and clearance. Ind. Eng. Chem. Res. 53, 9587-9599.

Tapp, H.S., Williams, R.A., 2000. Status and applications of micro electrical resistance tomography. Chem. Eng. Sci. 77, 119-125.

Tatterson, G. B., 1991. Fluid Mixing and Gas Dispersion in Agitated Tanks. McGraw-Hill, New York.

Tatterson, G.B., 1994. Scale up and design of industrial mixing processes. McGraw-Hill, New York.

Thompson, T.L., Clark, N.N., 1991. A holistic approach to particle drag prediction. Powder Technol., 67, 57-66.

Turton, R., Levenspiel, O., 1986. A short note on the drag correlation for spheres. Powder Technol., 47, 83-86.

Tyagi, M., Roy, S., Harvey III, A.D., Acharya, S., 2007. Simulation of laminar and turbulent impeller stirred tanks using immersed boundary method and large eddy simulation technique in multi-block curvilinear geometries. Chem. Eng. Sci., 62(5), 1351-1363.

Uchida, S., Okamura, S., Katsumata, T., Lida, K., 1989. Measurement of solids hold-up in a threephase fluidized bed by an ultrasonic technique. Chem. Eng. Sci. 44, 196-198.

Unadkat, H., Rielly, C.D., Hargrave, G.K., Nagy, Z.K., 2009. Application of fluorescent PIV and digital image analysis to measure turbulence properties of solid-liquid stirred suspensions. Chem. Eng. Res. Des. 87, 573-586.

Van den Akker, H.E.A., 2010. Toward a truly multiscale computational strategy for simulating turbulent two-phase flow processes. Ind. Eng. Chem. Res. 49, 10780-10797.

Van der Wielen, L.A.M., van Nunen, C.A.P.M., Luyben, K.Ch.A.M. 1998. Pulsed adsorbent transport in a countercurrent adsorptive reactor. AIChE J. 44 (1).

Vatanakul, M., Zheng, Y., Couturier, M., 2005. Ultrasonic technique for measuring phase holdups in multiphase systems. Chem. Eng. Commun. 192 (4-6), 630-646. 
Viergever, M.A., Todd-Pokropek, A.E., 1988. Mathematics and Computer Science in Medical Imaging. Springer, Germany.

Wang, M., Dorward, A., Vlaev, D., Mann, R., 2000. Measurements of gas-liquid mixing in a stirred vessel using electrical resistance tomography (ERT). Chem. Eng. J. 77, 93-98.

Wang, M., 2002. Inverse solutions for electrical impedance tomography based on conjugate gradients methods. Meas. Sci. Technol. 13, 101-117.

Wang, F., Jones, T.F., Williams, R.A., 2003. Visualisation of asymmetric solids distribution in horizontal swirling flows using electrical resistance tomography. Chem. Eng. Res. Des. , 81(A8), 854-861.

Wang, F., Mao, Z., Wang, Y., Yang, C., 2006. Measurement of phase-holdups in liquid-liquidsolid three-phase stirred tanks and CFD simulation. Chem. Eng. Sci., 61(22), 7535-7550.

Warsito, W., Fan, L.S., 2001. Measurement of real-time flow structures in gas-liquid and gasliquid-solid flow systems using electrical capacitance tomography (ECT). Chem. Eng. Sci. 56, 6455-6462.

Wells, B.E., Gauglitz, P.A., Rector, D.R., 2011. Comparison of waste feed delivery small scale mixing demonstration simulant to Hanford waste. Pacific Northwest National Laboratory PNNL20637 Rev.1.

Wen, C.Y., Yu, Y.H., 1966. Mechanics of fluidization. Chem. Eng. Prog. Symp. Ser. 62(62), 100111.

Wesseling, P., 2001. Principles of computational fluid dynamics. Springer, USA.

West, R.M., Jia, X., Williams, R.A., 1999. Quantification of solid-liquid mixing using electrical resistance and positron emission tomography. Chem. Eng. Comm. 175, 71-97.

White, M., Summerford, S.D. 1933. Studies in agitation, sand concentration as function of sand size and agitator speed. Ind. Eng. Chem. 1025-1027.

Wichterle, K., 1988. Conditions for suspension of solids in agitated vessels. Chem. Eng. Sci. 43 (3), 467-471. 
Williams, R.A., Beck, M.S., 1995. Process Tomography: Principles, Techniques and Applications. Butterworth- Heinemann, Oxford, UK.

Williams, R.A., Jia, X., McKee, S.L., 1996. Development of slurry mixing models using resistance tomography. Powder Technol. 87, 21-27.

Williams, R.A., Jia, X., West, R.M., Roberts, K.J., 1998. On line measurement of solids distributors in stirred tanks and crystallizers using electrical computed tomography. Int. Conf. on mixing and crystallization, April 22-25.

Wittmer, S., Falk, L., Pitiot, P., Vivier, H., 1998. Characterization of stirred vessel hydrodynamics by three- dimensional trajectography. Can. J. Chem. Eng. 76 (3), 600-610.

Wu, J., Zhu, Y.G., Pullum, L., 2002. Suspension of high concentration slurry. AIChE J. 48 (6), 1349-1352.

Xie, H.Y., Zhang, D.W., 2001. Stokes shape factor and its applications in the measurement of sphericity of non-spherical particles. Powder Technol. 114, 102-105.

Yang, L., Cheng, J., Fan, P., Yang, C, Mai, Z.S. 2012. Macromixing of solid-liquid systems in a stirred tank with double impellers. Chem. Eng. Technol. 36 (3), 443-449.

Yoshida, M., Kimura, A., Yoneyama, A., Tezura, S., 2012. Design and operation of unbaffled vessels agitated with an unsteadily forward-reverse rotating impeller handling solid-liquid dispersions. Asia-Pac. J. Chem. Eng. 7 (4), 572-580.

Yu, Z.Q., Chow, P.S., Tan, R.B.H., 2009. Residence time distribution of liquid and solid phases in a novel staged crystallizer. Ind. Eng. Chem. Res. 48, 10047-10054.

Zhang, X., Ahmadi, G., 2005. Eulerian-Lagrangian simulations of liquid-gas-solid flows in threephase slurry reactors. Chem. Eng. Sci., 60(18), 5089-5104.

Zheng, Y., Zhang, Q., 2004. Simultaneous measurement of gas and solid hold-ups in multiphase systems using ultrasonic technique. Chem. Eng. Sci. 59, 3505-3514.

Zwietering, T.N., 1958. Suspending of solid particles in liquid by agitators. Chem. Eng. Sci. 8 (34), 244-253. 